\title{
Calibration of Seismic Attributes for Reservoir Characterization
}

\author{
Final Technical Report \\ Reporting Period Start Date: October 1, 1998 \\ Reporting Period End Date: September 30, 2002 \\ Principal Author: Wayne D. Pennington \\ Date of Issue of Report: October 2002 \\ DOE Award Number DE-AC26-98BC15135
}

\author{
Submitted by: \\ Wayne D. Pennington \\ Department of Geological and Mining Engineering and Sciences \\ Michigan Technological University \\ 1400 Townsend Drive \\ Houghton, MI 49931
}

\author{
Authors: \\ Wayne Pennington \\ Horacio Acevedo \\ Aaron Green \\ Joshua Haataja \\ Shawn Len \\ Anastasia Minaeva \\ Deyi Xie
}




\section{Disclaimer}

This report was prepared as an account of work sponsored by an agency of the United States Government. Neither the United States nor any agency thereof, nor any of their employees, makes any warranty, express or implied, or assumes any legal liability or responsibility of the accuracy, completeness, or usefulness of any information, apparatus, product, or process disclosed, or represents that its use would not infringe privately owned rights. Reference herein to any specific commercial product, process, or service by trade name, trademark, manufacturer, or otherwise does not necessarily constitute or imply its endorsement, recommendation, or favoring by the Untied States Government or any agency thereof. The views and opinions of authors expressed herein do not necessarily state or reflect those of the United States Government or any agency thereof. 


\begin{abstract}
The project, "Calibration of Seismic Attributes for Reservoir Calibration," is now complete. Our original proposed scope of work included detailed analysis of seismic and other data from two to three hydrocarbon fields; we have analyzed data from four fields at this level of detail, two additional fields with less detail, and one other 2D seismic line used for experimentation. We also included time-lapse seismic data with ocean-bottom cable recordings in addition to the originally proposed static field data. A large number of publications and presentations have resulted from this work, inlcuding several that are in final stages of preparation or printing; one of these is a chapter on "Reservoir Geophysics" for the new Petroleum Engineering Handbook from the Society of Petroleum Engineers.

Major results from this project include a new approach to evaluating seismic attributes in timelapse monitoring studies, evaluation of pitfalls in the use of point-based measurements and facies classifications, novel applications of inversion results, improved methods of tying seismic data to the wellbore, and a comparison of methods used to detect pressure compartments. Some of the data sets used are in the public domain, allowing other investigators to test our techniques or to improve upon them using the same data.

From the public-domain Stratton data set we have demonstrated that an apparent correlation between attributes derived along 'phantom' horizons are artifacts of isopach changes; only if the interpreter understands that the interpretation is based on this correlation with bed thickening or thinning, can reliable interpretations of channel horizons and facies be made. From the publicdomain Boonsville data set we developed techniques to use conventional seismic attributes, including seismic facies generated under various neural network procedures, to subdivide regional facies determined from logs into productive and non-productive subfacies, and we developed a method involving cross-correlation of seismic waveforms to provide a reliable map of the various facies present in the area. The Wamsutter data set led to the use of unconventional attributes including lateral incoherence and horizon-dependent impedance variations to indicate regions of former sand bars and current high pressure, respectively, and to evaluation of various upscaling routines. The Teal South data set has provided a surprising set of results, leading us to develop a pressure-dependent velocity relationship and to conclude that nearby reservoirs are undergoing a pressure drop in response to the production of the main reservoir, implying that oil is being lost through their spill points, never to be produced. Additional results were found using the public-domain Waha and Woresham-Bayer data set, and some tests of technologies were made using 2D seismic lines from Michigan and the western Pacific ocean.
\end{abstract}




\section{Table of Contents}

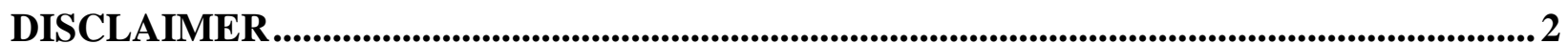

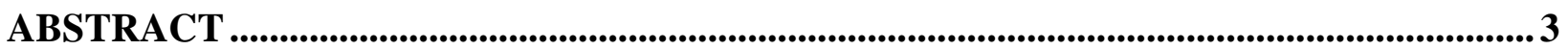

TABLE OF CONTENTS............................................................................................................................ 4

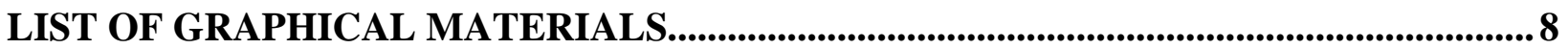

EXECUTIVE SUMMARY ......................................................................................................................... 15

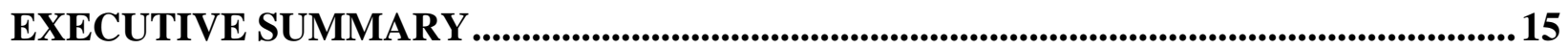

INTRODUCTION............................................................................................................................................. 17

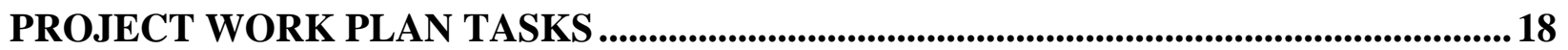

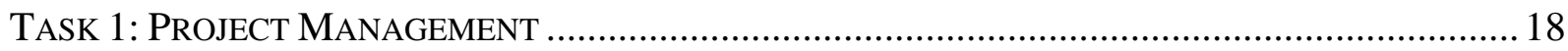

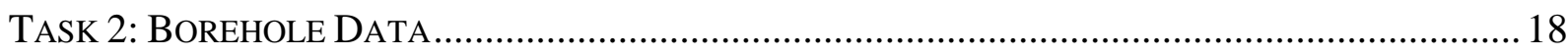

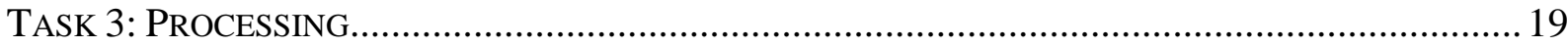

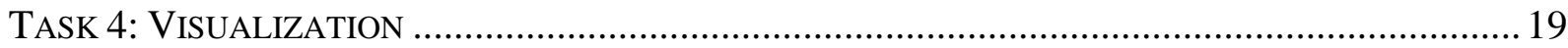

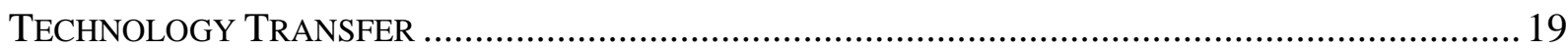

SUMMARY OF RESULTS...........................................................................................................................20

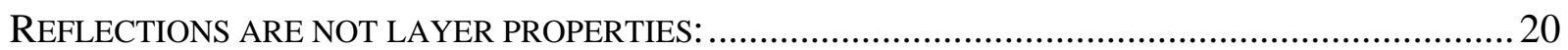

POINT MEASUREMENTS SHOULD NOT BE MADE OFF OF SEISMIC PEAKS OR TROUGHS:..................20

FREQUENCY-DOMAIN AND TIME-DOMAIN STUDIES SIMPLY DESCRIBE THE WAVESHAPE: :............20

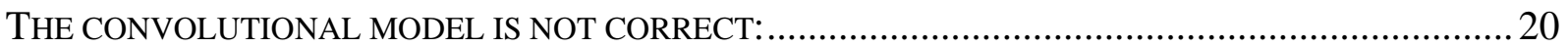

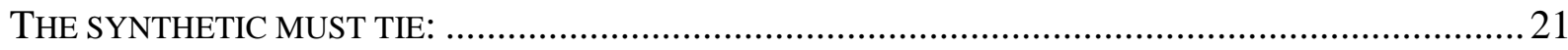

MULTIPLE ATTRIBUTES CAN HELP, OR THEY CAN HINDER: ..................................................... 21

HOW CONSTRAINED MUST WE BE? HOW DETAILED SHOULD OUR RELATIONSHIPS BE? ...............21

WHAT LEVEL OF ROCK-PHYSICS UNDERSTANDING IS REQUIRED? .............................................21

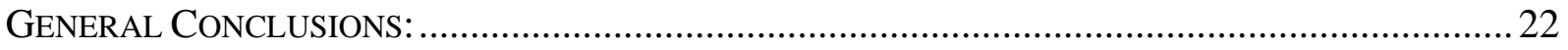

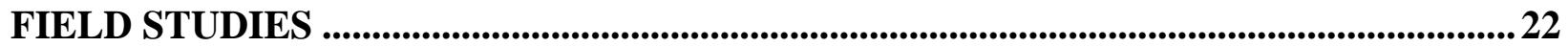

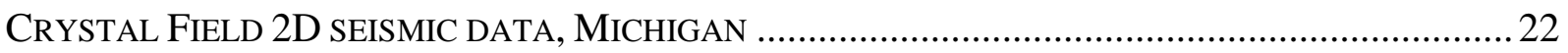




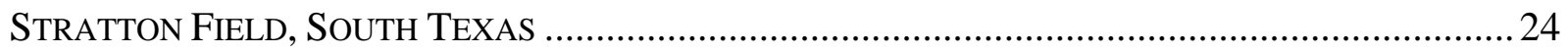

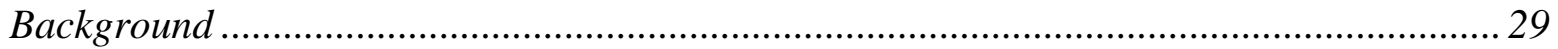

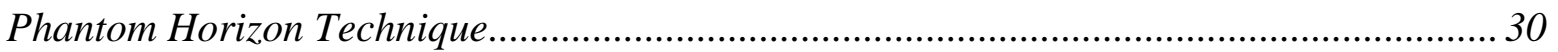

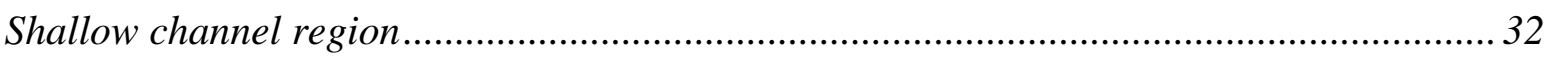

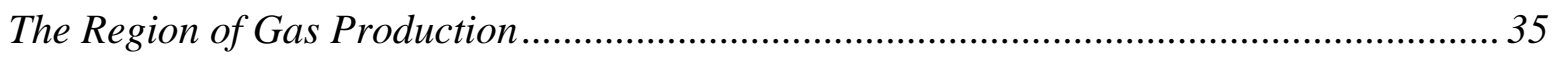

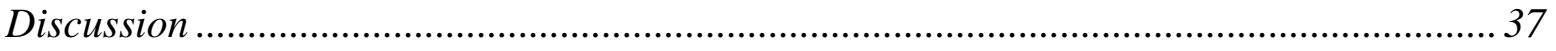

Conclusions Based on Stratton Field Study ………….................................................... 38

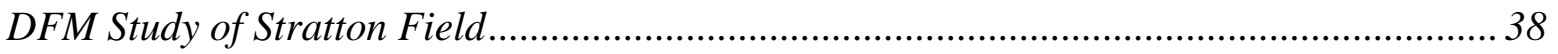

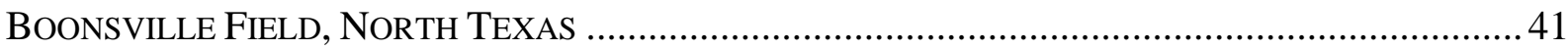

BOONSVILLE FIELD 1: QUANTITATIVE SEISMIC FACIES ANALYSIS FOR THIN-BED RESERVOIRS ... 43

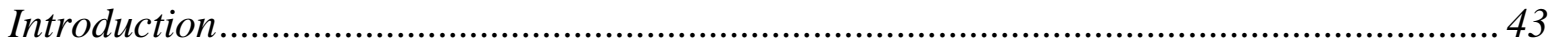

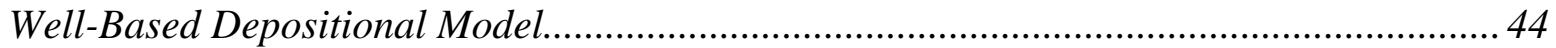

Seismic trace pattern and Depositional Model .................................................................. 46

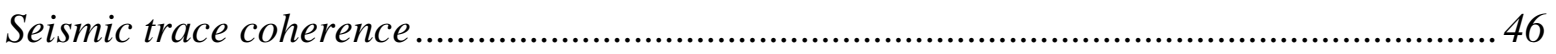

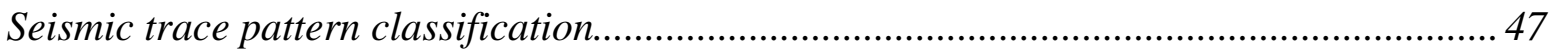

A New Agorithm for Seismic Facies Analysis .................................................................. 48

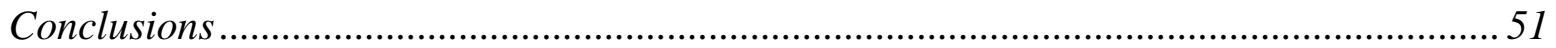

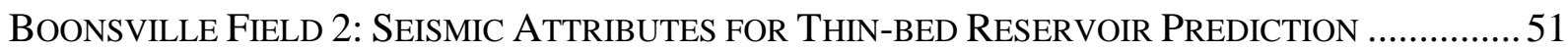

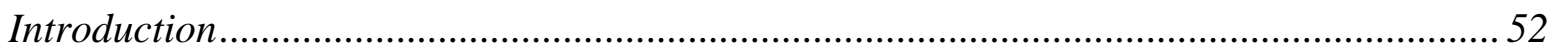

Evaluation of the Conventional Thin-Bed Tuning Model ...................................................... 53

Generalized Regression Neural Network Inversion......................................................... 56

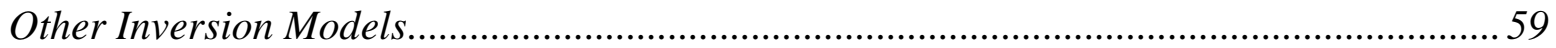

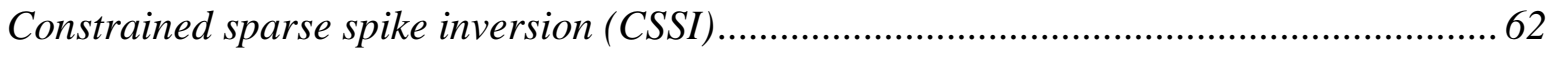

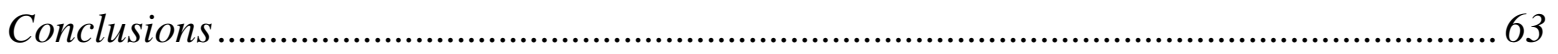

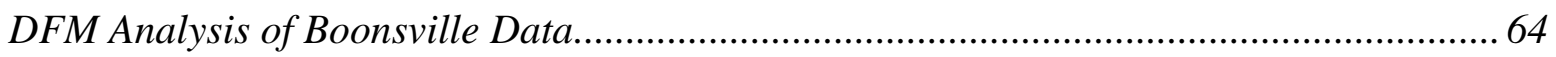

EVALUATION OF SEISMIC ATTRIBUTES IN WAMSUTTER FIELD, WYOMING...................................66

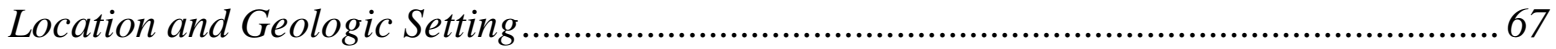

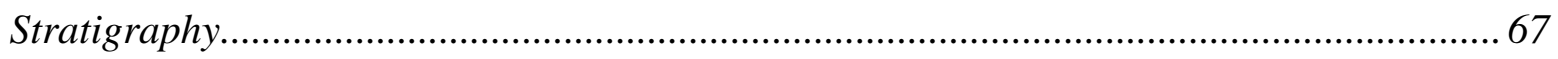

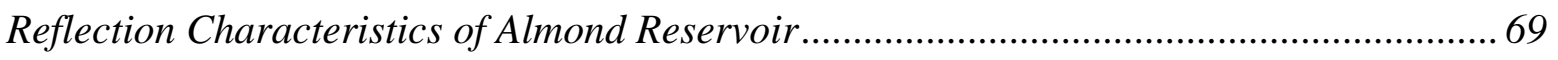

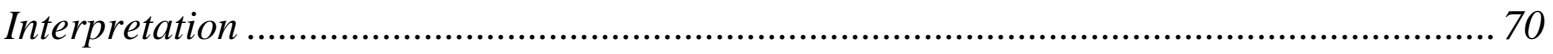

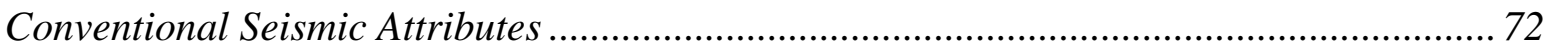

Time Derived Horizon Attributes ..................................................................................... 72 
Window or Trace Based Attributes ........................................................................... 73

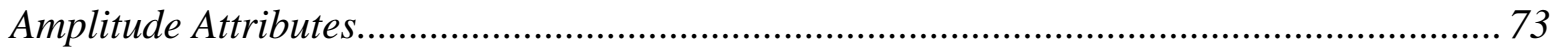

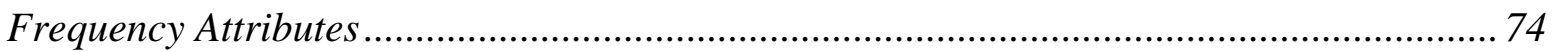

Seismic Inversion for Acoustic Impedance ................................................................... 74

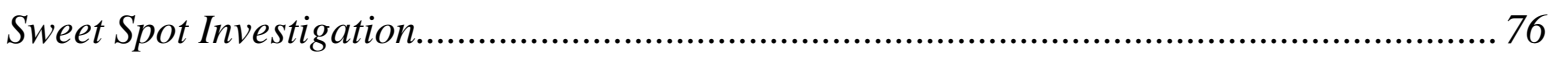

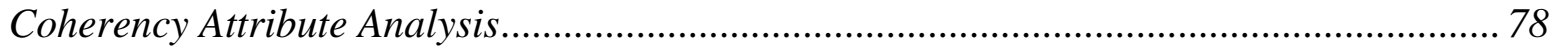

Dynamic Fluid Method at Wamsutter:........................................................................ 81

One-dimensional synthetic seismograms generated using Backus averaging.................... 84

TEAL South Time-LAPSE Study, Gulf OF MeXico.......................................................... 97

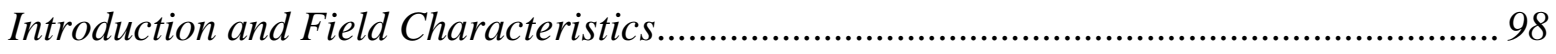

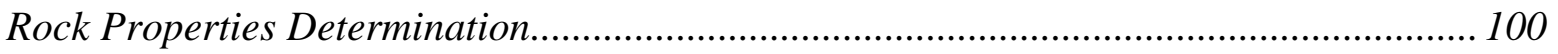

Pre-Stack Time-Lapse Seismic Observations............................................................. 103

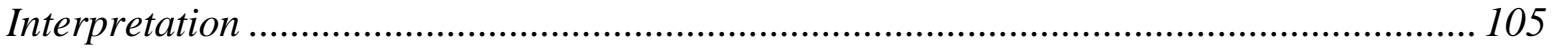

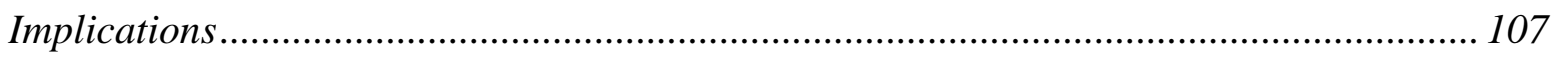

EFFECTIVE PRESSURE CHANGES AND SEISMIC VELOCITIES...........................108

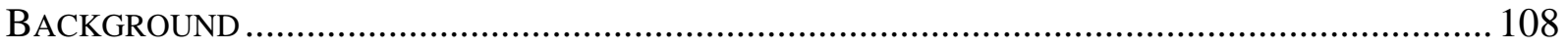

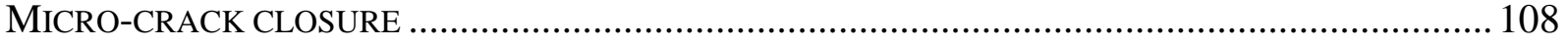

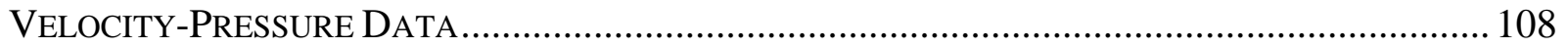

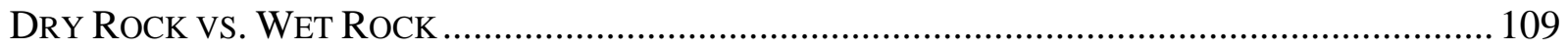

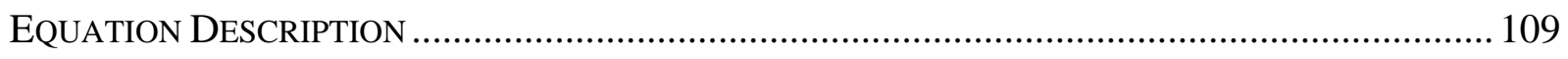

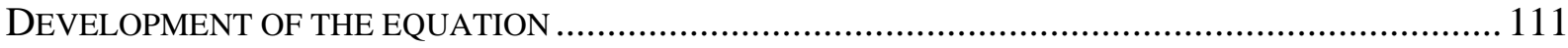

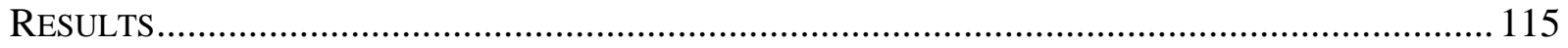

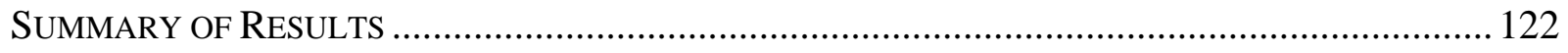

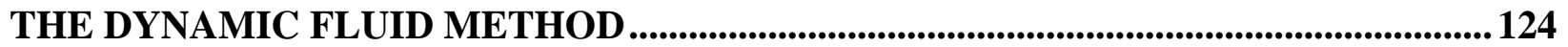

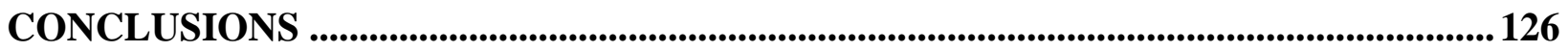

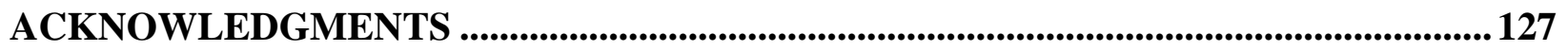

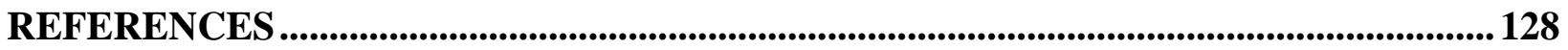

LIST OF ACRONYMS AND ABBREVIATIONS .....................................................133 
DISCLAIMER

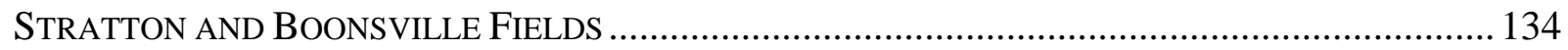

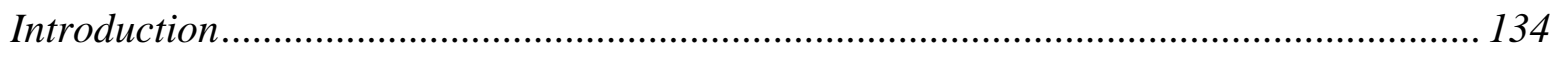

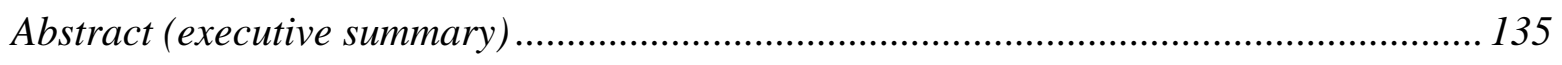

Processing and interpretation of seismic data in DFM technologies (general information)

Processing and interpretation of 3D seismic data in the area of Stratton Field ................ 145

Processing and interpretation of seismic data in Boonsville field................................... 153

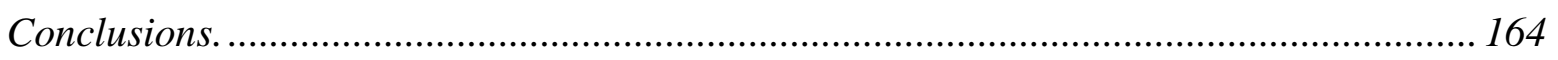

Recommendations for further analysis.................................................................. 165

DYNAMIC Fluid METHOD (DFM) INTERPRETATION OF THE OIL AND GAS POTENTIAL OF THE

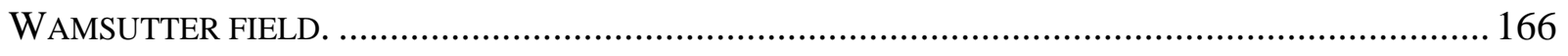

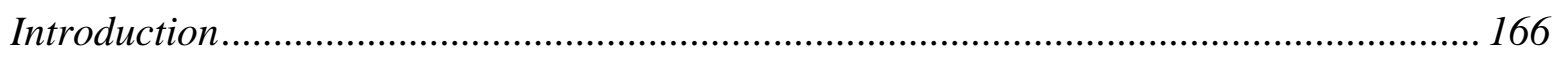

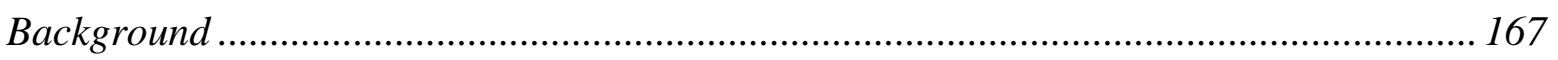

DFM interpretation of seismic data .................................................................. 170

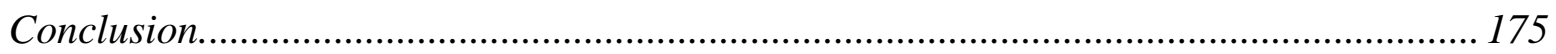

The Dynamic Fluid Method (DFM) ANAlysis of SEISMic DATA IN THE AREA OF WaHA

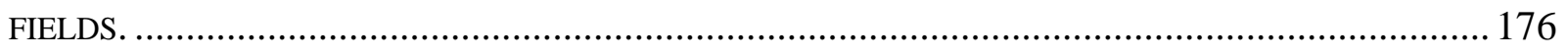

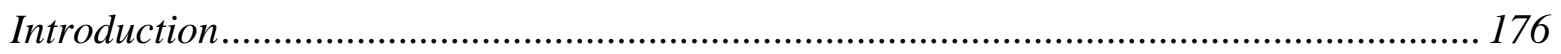

The methodology of DFM analysis and the results of prediction of the scheme of block dynamics in the Waha fields................................................................................... 177

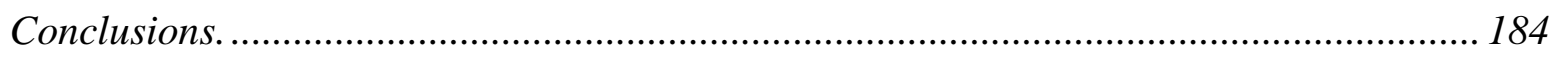

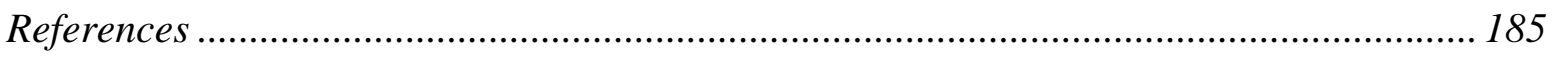




\section{List of Graphical Materials}

Figure 1: Left side: A shot gather of the Crystal field data; Right side: a CMP gather............. 23

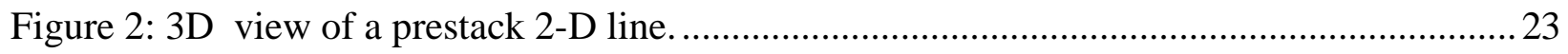

Figure 3: Seismic gather from the Pacific Ocean............................................................ 24

Figure 4: Shallow horizon in Stratton data showing apparent stream channels.........................25

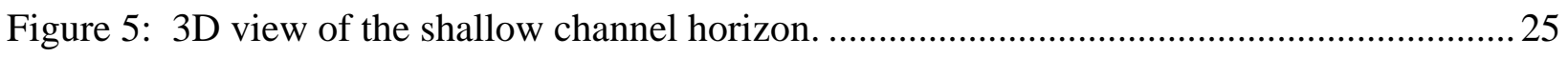

Figure 6: Schematic diagram summarizing approach used by the Texas BEG in Stratton field.. 26

Figure 7: Stratton data picked with slightly differing constraints (in blue and green), using nearest peak or autocorrelation maximum (left: general view; right: close-up view). ........ 26

Figure 8: A comparison of facies classification based on the differently-picked horizons. ........27

Figure 9: Seismic facies classification map of an interval near $840 \mathrm{~ms}$ in the Stratton data set... 27

Figure 10: Cut-out region of channel, with detailed reclassification of facies within this channel. .28

Figure 11: A combination of time structure and facies classification. in the Stratton data set.....28

Figure 12 Location of the Stratton Field (adapted from Hardage 1995)................................... 29

Figure 13 Stratigraphic sequence of the Oligocene ..........................................................29

Figure 14 Seismic section showing the two horizons studied: a channel region at approximately $842 \mathrm{~ms}$ and the primary producing region at approximately $1640 \mathrm{~ms}$ in time. The heavily faulted area is due to a series of growth faults in the Vicksburg formation........................ 30

Figure 15: Calculating normalized trace gradients for the facies classification. ........................ 31

Figure 16: The shallow channel region at approximately $842 \mathrm{~ms}$ in time. A phase change occurs (from light to dark gray) across the image due to the gentle dip of the horizon.................. 32

Figure 17: Structural (time) surface of the picked horizon outlining the channel. ..................... 33

Figure 18: The phantom horizon created by subtracting $101 \mathrm{~ms}$ from reference horizon A........33

Figure 19: Attributes of the phantom horizon corresponding to the shallow channel................ 34

Figure 20: Unsupervised facies classification of the shallow channel region............................ 34

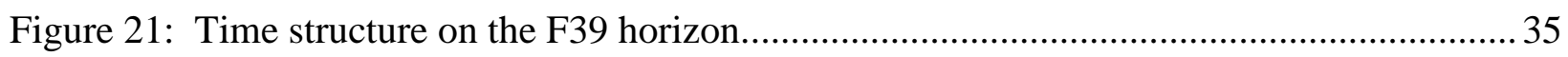


Figure 22: Attributes on the phantom horizon corresponding to the F39 level. 36

Figure 23: Seismic facies classification of the F39 Phantom horizon. 36

Figure 24: Horizons associated with tracking F39. 37

Figure 25: Isopach of time separation between the F11 horizon and the tracked F39 horizon. ... 37

Figure 26: DFM results for Stratton field along one inline, showing regions of low effective pressure in red. 39

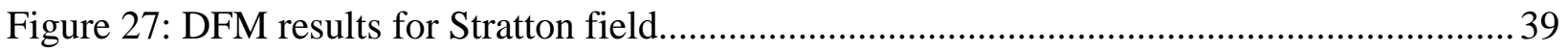

Figure 28: DFM results for Stratton field showing the block structur. 40

Figure 29: DFM results for Stratton field showing regions of low effective pressure as purple 3D bodies. 40

Figure 30a: Location map of the Boonsville study area.................................................. 41

Figure 30b. Traverse seismic line through the main delta showing the Caddo sequence............ 41

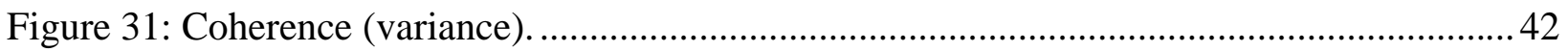

Figure 32: 3D visualization of the Boonsville data set ..................................................... 42

Figure 33: Sample impedance contrasts in Boonsville field.............................................. 43

Figure 34: Generalized well log trends and depositional subfacies and lithologies of the Caddo

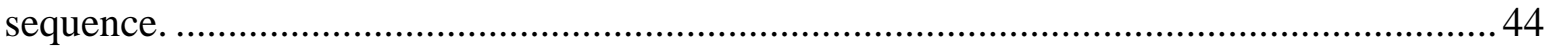

Figure 35: Representative well log trends and depositional subfacies of the Caddo sequence .... 45

Figure 36: Well correlation and interpretation; flattened on the Bottom Caddo marker............. 45

Figure 37: Seismic coherence time slice at $890 \mathrm{~ms}$ within the Caddo sequence. .......................47

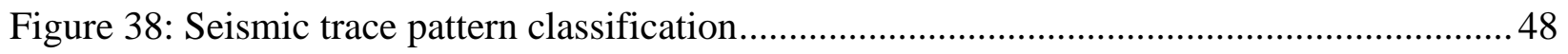

Figure 40: The combined seismic facies using multiple traces............................................... 51

Figure 41: Conventional thin-bed tuning model with notations for our data set. ......................53

Figure 42: Modeled seismic response showing amplitude attenuation where two closely-spaced

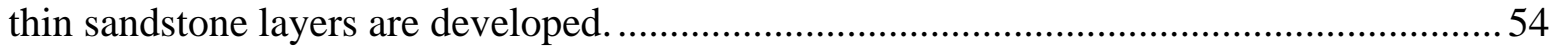

Figure 43: Seismic features through the main delta system showing amplitude decrease and wavelet doublet in interdelta subfacies. .................................................................. 54 
Figure 44: RMS Amplitude plotted against summed thickness of sandstone and limestone. ......55

Figure 45: Inverted impedance curves using the GRNN model at 36 wells.

Figure 46: Crosslines 171 (a) and 125 (b) of the inverted impedance data using the GRNN model indicating sandstone and limestone as higher impedances. ........................................ 58

Figure 47: Inverted impedance volume (GRNN model) with different opacity cut-offs.............59

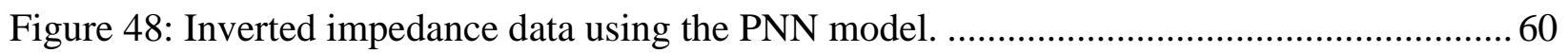

Figure 49: Crossline 117 of the invertedimpedance volume using the PNN model................... 60

Figure 50: Actual sandstone and limestone thickness plotted against predicted thickness from seismic inversion (PNN model)

Figure 51: Estimated sandstone thickness of Caddo main delta system (PNN model) .....

Figure 52: Traverse line showing the inverted impedance using CSSI model and agreement with well data (the five wells indicated were used to estimate the wavelet and provide constraints). 62

Figure 53: Inverted impedance volume using the CSSI with different opacity cut-offs. 63

Figure 54: DFM results for the Caddo interval (red represents decompressing regions). The dashed lines indicate block boundaries interpreted by TransSeismic from the data. The size of the diamond symbols is proportional to the oil recovery from the Caddo interval from wells in the field. 64

Figure 55: Three-dimensional image of the DFM results for the Boonsville data set, processed by TransSeismic and imaged at MTU; the red colors represent areas of decreasing effective pressure, presumed to have greater potential for hydrocarbon accumulations in the DFM approach.

Figure 57: Location of the Wamsutter survey area. After Stephenson et al, 1987, and Keighin et al, 1989. .67

Figure 58: Schematic cross-section (after Roehler, 1990), showing the Wamsutter area of study. .68

Figure 59: Location of the seismic survey outlined in red and first-year (gas) production indicated by size of bubbles. 68

Figure 60: Typical logs for the Wamsutter area.

Figure 61: Typical logs of the portion of the Wamsutter field where the sand-bar or barrier-island facies is present. 70 
Figure 62: Seismic section through the data volume,with inset map showing general dip ......... 71

Figure 63: Seismic section in area of the western sand-bar or barrier-island facies.................. 71

Figure 64: Dip map with time contours superimposed. .................................................... 72

Figure 65: Dip-azimuth map with coloring emphasizing two dip directions............................ 72

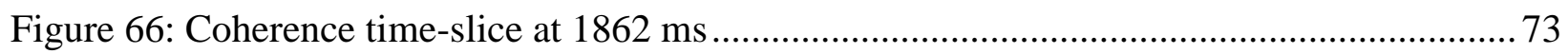

Figure 67: Maps of two amplitude-based attributes........................................................ 74

Figure 68:Profile through a well-section of the inverted volume. ........................................75

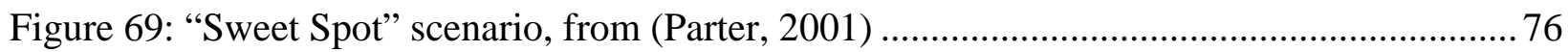

Figure 70: A portion of the inverted volume with specific horizons indicated.......................... 77

Figure 71: Extraction of low-impedance bodies within small intervals. ................................ 78

Figure 72a: Seismic section in area of the barrier island or sand bar; its location is circled. ....... 79

Figure 72b: Variance (incoherency) attributes over several horizon-based windows as labeled in

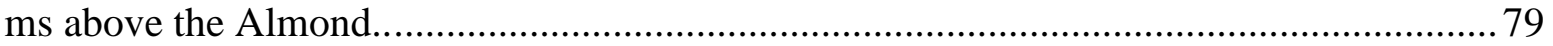

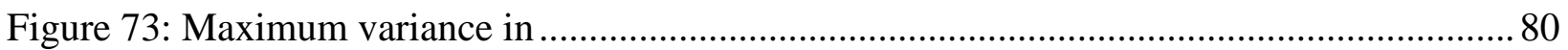

Figure 74: Energy half-time (left) and sum-of-magnitudes amplitude (right) shown with first-

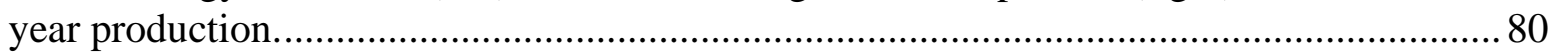

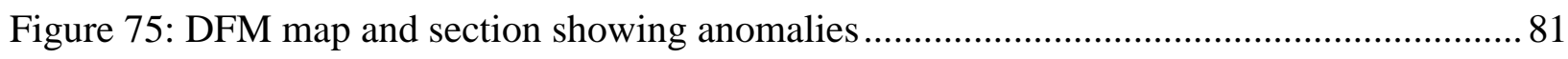

Figure 76: DFM section showing the Lewis shale region between Lance and Almond formations. 82

Figure 77: 3D view of the most extreme values of pressure attribute from DFM..................... 82

Figure 78: Collected bodies of anomalous pressure values from DFM analysis...................... 83

Figure 79: Summed values of the DFM anomalies and the RMS amplitudes of these summed bodies over the eastern portion of the survey area........................................................ 83

Figure 80: Comparison of the summed low-acoustic-impedance values and the DFM results... 84

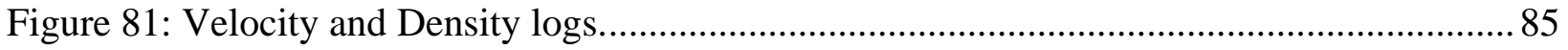

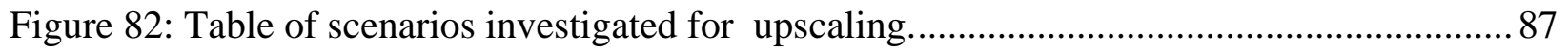

Figure 83: Relationship between density and velocity in coals, based on core measurements. ... 87 
Figure 84: Calculated modulus (left side, red) and Backus-averaged values (black); computed velocity (right side, green) and Backus-averaged (black)................................................... 88

Figure 85: Comparison of original and Backus-averaged time-depth relations............................ 88

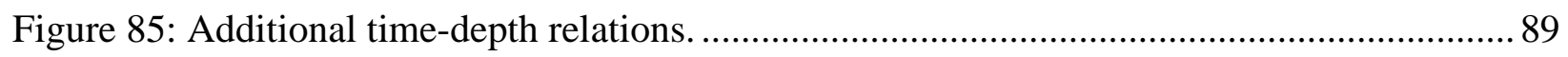

Figure 87: "High-Fidelity” Backus-averaging ........................................................................... 90

Figure 88: Relationship between period or frequency and wavelength....................................... 91

Figure 89: Amplitude spectrum (purple) of seismic data at a well location, 1000-2000 ms. Estimated wavelet spectrum (blue), ............................................................................ 91

Figure 89: Wavelets used for synthetic seismograms and spectral balanced velocity functions. . 92

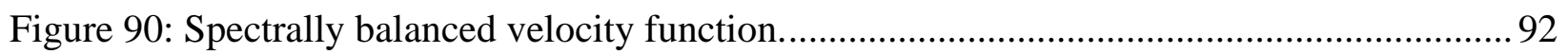

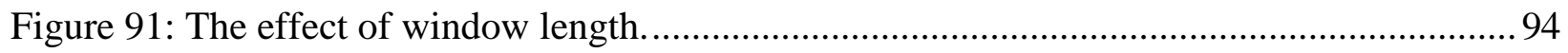

Figure 92: Drift curves corresponding to different window sizes (left panel)..............................94

Figure 93: Correlation coefficients for synthetic seismograms from Hi-Fi Backus averaged velocity functions..........................................................................................................95

Figure 94: Synthetic seismograms (blue) generated using "high fidelity Backus averaged"

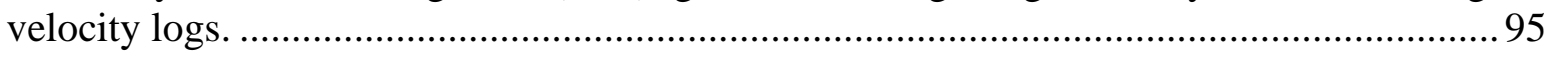

Figure 95: Comparison of conventional synthetics and synthetic seismogram created using Hi-Fi Backus-averaged velocity log. Synthetic traces are shown in blue, seismic trace in red...... 96

Figure 96: Three-dimensional image of a time-lapse difference volume found by Texaco during the first phase of the Teal South project. Bright colors indicate a change in reflection amplitude. From Ebrom et al........................................................................................... 97

Figure 97: Acoustic impedance map view of the target horizon at Teal South............................ 98

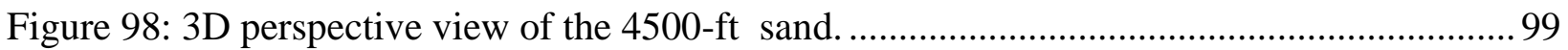

Figure 99: Smoothed production history of the 4500-ft reservoir. ……................................... 100

Figure 100: Inverted (legacy data) of the reserovoir interval.................................................... 101

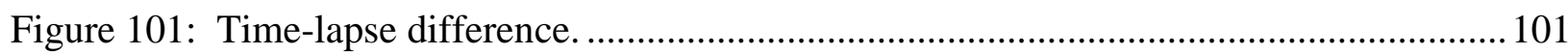

Figure 102: Changes in P-wave velocity (Vp), Poisson's ratio (PR), and Acoustic Impedance

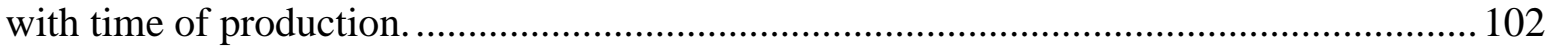


Figure 103: Predictions for Amplitude-versus-Offset effect to be observed in time-lapse data for Teal South. Small squares indicate each additional 5 degrees in angle of incidence, and the black square indicates 30 degrees. 103

Figure 104: Amplitudes versus offset in the time-lapse data for the producing reservoir.......... 104

Figure 105: Amplitudes versus offset for the nearby unproduced reservoirs. 104

Figure 106: Traverse through the inverted acoustic impedance volume from the legacy data set. 105

Figure 107: Cartoon schematic of the sequence of events resulting in the loss of hydrocarbons from the Little Neighbor due to production in the 4500-ft reservoir. 106

Figure 108: Time-lapse difference image of the $4500-\mathrm{ft}$ sand, showing the relative brightness of the nearby reservoirs and the apparent path of pressure migration. 107

Figure 109: Sharp velocity increases are shown on the left of the graph, due to cracks closing. The velocity increase to the right is very gradual, as the remaining ellipsoids slowly close with pressure. 108

Figure 110: Velocity predictions tied at a point are more accurate than general velocity curves 110

Figure 111: Fitting $P_{e}-e^{-D P e}+1$ to velocities to solve for coefficient A and B. Coefficient D was varied until the least $R^{2}$ value was found. 111

Figure 112: These plots show that there is no correlation between clay or porosity and coefficient A, B or D. These are the same results that were found by Eberhart-Phillips 112

Figure 113: Best fit coefficient D values show no relation to velocity measurements in Han's data set....... 112

Figure 114: Best-fit coefficient A values show a strong correlation with measured velocity. This means if we start with a known velocity, we can predict coefficient A.

Figure 115: Best-fit coefficient B values show a weak correlation with measured velocity. Though there may be a trend of decreasing velocity with increasing coefficient $\mathrm{B}$, velocity alone is not useful in predicting coefficient $\mathrm{B}$ values. 113

Figure 116: Best-fit coefficient B values show a strong correlation with change in measured velocity. This means if we can solve for coefficient $\mathrm{B}$, we can predict what the change in velocity is. 113

Figure 117: Slope and intercept in the coefficient A vs. velocity best fit line. 113 
Figure 118: Plot of Table 2, giving the equation that will predict the slope in the best fit line that relates coefficient $\mathrm{B}$ and pressure change. Note that the data points for changes between two pressures are plotted at the midpoint of these two pressures. 114

Figure 119: This is simply an example of integrating the equation in figure 11 above, and applying it at different pressures. 114

Figure 120: Plot of data for sample 49. 116

Figure 121: Actual values in Han's dry P-wave data set vs. values predicted by my method, assuming a known velocity at .20 kbar. This is for every sample in the entire data set. ... 116

Figure 122: Plot of data for sample 45. 116

Figure 123: Actual values in Han's dry P-wave data set vs. values predicted by my method, assuming a known velocity at .50 kbar. This is for every sample in the entire data set. ... 117

Figure 124: Actual values in Freund's dry P-wave data set vs. predicted values, assuming a known velocity at $.24 \mathrm{kbar}$. This is for pressures $.08, .40$ and .60 for every rock sample in the entire data set. 118

Figure 125: Actual values in Freund's dry P-wave data set vs. predicted values, assuming a known velocity at $.60 \mathrm{kbar}$. This is for pressures $.08, .24$, and .40 for every sample in the entire data set.. 118

Figure 126: Changes between two pressures for the average velocities of the entire Han data set. Coefficient $1_{\alpha}$ is .0366 and $m_{\alpha}$ is -.0004 . 119

Figure 127: Comparing velocity change predictions between our new method and Bentley's method. 120

Figure 128: For this typical high velocity sample, Bentley's method predicts velocity changes more accurately than our new method. The new method predicts values that are too low for the slope of the curve at high velocities.

Figure 129: The typical medium velocity sample shows that the new method is more accurate than Bentley's method in this situation.

Figure 130: The new method predicts the actual velocity values even more accurately at this typical low velocity value. 121

Figure 131: Clay Content vs. residual error for Freund's P-wave data is shown here. Samples with clay content below about 30 percent show a much higher error. 122

Figure 132: Clay Content vs. $\mathrm{R}^{2}$ error (residual) for Han's P-wave data is shown here. Unlike the Freund data set, the magnitude of error does not seem to be affected by clay content. 122 


\section{Executive Summary}

The project, "Calibration of Seismic Attributes for Reservoir Calibration," is complete, having expanded our original scope considerably beyond that which was proposed. We have applied conventional and new methods of analysis and interpretation to four main data sets, including one time-lapse data set, and tested some specific technologies on additional data.

The main results of the project can be classified along these lines:

- Pitfalls (how to use 'phantom' horizons carefully)

- Unconventional attributes

- Lateral extent of incoherence

- Cross-correlation with 'type' seismograms

- Impedance variation within specific layers

- Detection techniques for pressure compartments and fluid migration

- Upscaling from sonic to seismic

- Methodology

- Recommendations for routine implementation

- Pressure-dependence of elastic properties

- New relations based on dry-frame laboratory measurements

- Importance of inclusion in time-lapse studies

- Reservoir behavior detected from time-lapse seismic observations

The public-domain Stratton data provided a challenge in thin-bed reservoir characterization in the absence of sonic-log calibration data. In general, the seismic character of potential productive zones is obscure in this data set, and horizons containing the pay zones are typically discontinuous. Previous work by other authors has demonstrated the apparent usefulness of simple attributes mapped along 'phantom' horizons which were tied at one well through a VSP and controlled by a constant offset from a nearby continuously tracked horizon. We have demonstrated in this project that this apparent correlation is often an artifact of isopach changes, which dominate the interpretations based on simple attributes and on seismic facies analysis. However, if the interpreter understands that the interpretation is based on this correlation with bed thickening or thinning, reliable interpretations of channel horizons and facies can be made.

The public-domain Boonsville data set provided another challenge in thin-bed reservoir characterization, in which the seismic character of a productive sand zone appears to be indistinguishable from that of a non-productive limestone. In this case, the interplay of impedance and thickness conspire with tuning to produce the similarity in most attributes. This problem was attacked with two methods. In the first method, a technique was developed that made use of the well-log interpretations to divide the area into a number of facies, following a reasonable geological model; then the seismic attributes, including seismic facies generated under various neural network procedures, were used to further subdivide those regional facies into productive and non-productive subfacies. In the other method, a new technique involving cross-correlation of seismic waveforms was developed to provide a reliable map of various 
facies present in the area; we think this technique holds great promise for other data sets as well, and it appears to be extremely robust.

The Teal South time-lapse seismic data set has provided a surprising set of data, extremely rich in interpretation possibilities. We used the limited log data and excellent seismic data of this classical bright-spot reservoir in the Gulf of Mexico to develop a robust seismic petrophysics model through waveform (stratigraphic) inversion for acoustic impedance. We then used this model, together with a pressure-dependent elastic modulus relationship, to predict the future seismic response of the reservoir, as it was being produced. Our predictions met the observations with uncanny accuracy. But observations of nearby, unproduced reservoirs also indicated a similar response, one that was not predicted with classical reservoir models or simulations. We concluded that these nearby reservoirs are undergoing a pressure drop in response to the production of the main reservoir, and that oil is being lost through their spill points (as gas comes out of solution), never to be produced. This set of observations may have serious ramifications for engineering and exploitation techniques throughout the Gulf of Mexico.

The Wamsutter data set provided our most-challenging opportunities. This thin bedded lowporosity sand within the Almond formation of the western US has demonstrated to other studies that conventional seismic attributes are not typically useful in identifying depositional facies or productive zones. We sought unconventional attributes that were designed to identify those features which we concluded should be present in the seismic data as a result of knowing the rock physics associated with production. We found that a measure of lateral incoherence applied along a phantom horizon, designed to track a sand bar and its distal marine equivalent, provided extraordinary correlation with productivity from that sand bar. We also found that a technique we developed to identify high pressure from impedance variations along layers correlates strongly with results from the DFM technique of TransSeismic International and may indicate pressure compartmentalization not associated with the sand bar; our interpretation is that these techniques are indicating zones of high fluid pressure and/or microfracturing. We also tested a variety of techniques of upscaling sonic and density logs to the seismic scale using a thin-layer effective medium model, and have significantly improved the tie between synthetic seismograms and real seismic data in this data set.

As part of this project, seismic data from four areas was provided to a private company, TransSeismic International, for their proprietary processing. The Stratton, Boonsville, Waha (Woresham-Bayer, West Texas), and Wamsutter data sets were used; the first three of these are public-domain data sets, so others can investigate the results independently. Comparison of our results with those provided by TSI is provided in context in this report. 


\section{Introduction}

The objectives of this project were three-fold:

- To determine the physical relationships between seismic attributes and reservoir properties in specific field studies;

- to improve the usefulness of seismic data by strengthening the physical basis of the use of attributes; and,

- in the third year of the project, to test the approaches suggested or developed during the first two years on at least one new data set.

This report first briefly summarizes the results of the various tasks of the project, following the Project Work Plan. Then it describes the results of our analyses of each data set in some detail. Finally, it summarizes the results and conclusions. An appendix contains four reports provided by TransSeismic International, describing their DFM methodology and results of their studies on four data sets.

Seismic attributes are used in a number of ways. Currently, the most commonly used attribute is the simple seismic trace amplitude along a specific horizon or time slice. The amplitude, of course, is most closely associated with the actual reflection coefficient (for a zero-offset trace), and is therefore indicative of the contrast in acoustic impedance above and below the reflecting horizon. Usually, the amplitude is taken at the maximum peak or trough, and is unambiguous in its definition. Sometimes, however, in an effort to increase resolution, the amplitude is taken at some other point in the waveform, and this has led to apparent additional insight. We will argue in this report that this insight is misleading, and that such intermediate amplitudes are not reliable measures of layer or reflecting-horizon properties. There is a large number of additional attributes that are less commonly used in the industry today, although they all have certain advantages. The reflection strength, instantaneous amplitude, or envelope amplitude have removed the effect of internal zero-crossings within a wavelet, and provide a more-robust measure of true reflecting properties. Taken one step further, the integration of reflection strength should provide insight into true bed properties, rather than interface properties, but the band-limited nature of seismic data prevents this simple application. Instead, an inversion process is required, generally incorporating well-log measurements to provide the background trends of acoustic impedance.

Additional techniques have been used to classify reflections or seismic intervals into groups, or facies. These usually incorporate a sort of neural network analysis due to the complexityof the problem, but they may also include other types of clustering analysis or statistical treatments. While these techniques can often provide extremely useful insight into correlations that were not immediately apparent to the eye, they should be used with great care in order to obtain the physical insight sought in exploration and field development.

In general, we have advocated using a physics-driven approach to seeking appropriate seismic attributes for use in any specific study. That is, one should try to determine, using knowledge of rock physics and the seismic data available, which attributes are best-suited to observe those features of most importance. Sometimes these attributes may be conventional ones, such as amplitude or acoustic impedance; but in difficult cases, the attributes will turn out to be unconventional ones, and must be generated specifically for that data set. 
This project has been a series of experiments with conventional attributes and unconventional, specialized, ones. The data sets chosen for study were for the most part chosen to be typical examples of data used by domestic producing companies; they were never complete in the sense of having all the data needed for a truly scientific study, but they were realistic. We have found a number of interesting results, including pitfalls and recommended practices, and have extended the study to include time-lapse seismic observations.

The studies by TransSeismic International (TSI) were contracted in order to provide an opportunity to investigate specific case histories of their processing and interpretation methods, called DFM, for Dynamic Fluid Method. This model assumes that most hydrocarbon traps occur as a result of fluid movements driven by vertical compaction (or compression) and decompression, which in turn provide pressure gradients and perhaps microfracturing. The DFM method is intended to identify those regions which are in a compressing or decompressing state, leading to interpretations of fluid migration patterns. TSI processed and interpreted three of the data sets that were used in the main part of the study, and one other public-domain data set chosen for its structural elements in a hard rock, which we thought would be an optimal location for this application.

\section{Project Work Plan Tasks}

\section{Task 1: Project Management}

Project management encompassed reporting and project support. Reporting has been on schedule. Project support remained on schedule after some initial redirection of efforts during the first year (resolved by the hiring of additional support personnel in systems administration).

Training for some of the software packages was accomplished by attending training courses conducted by the companies providing the software. Members of our team take part in training on the following packages:

- GeoQuest GeoFrame products from Schlumberger

- IC2 from Scott Pickford

- Stratimagic from Paradigm Geophysical

- Jason GeoScience Workbench from Jason Geosystems

- ProMax seismic processing software from Landmark Graphics Corporation

System administration support training has been taken in the following software suites:

- Geoframe from Schlumberger

- Landmark Openworks from Landmark Graphics Corporation

We also use GXTechnology products, Seismic Micro-Technology, and Hampson-Russell products.

\section{Task 2: Borehole Data}

Borehole data for this project consists of three types: existing log data, existing core data, and new core data. We have made use of all existing data, and have obtained new information on 
well-completion histories in Stratton field for help in identifying productive intervals in this complex field. We made new measurements on existing core from the Wamsutter area to help in characterizing the thin-bed nature of the formation. And we made novel use of inverted seismic data to constrain rock properties where log data was incomplete. Log data was used for every field study. Production data was used in some of the studies.

\section{Task 3: Processing}

We have conducted most of the processing of data that is required at MTU using software provided by donors; additional processing (for DFM) has been conducted by a subcontractor.

Our processing efforts have included the following:

- prestack processing of multiple 2D lines to evaluate acquisition artifacts,

- poststack processing for attribute extraction,

- poststack processing for variance/coherence,

- poststack inversion for acoustic impedance, and

- synthetic seismogram generation.

New routines were written using MatLab, $\mathrm{C}++$, or other languages appropriate to the task. In addition, analysis of data included identification of horizons, facies, and other attributes, all of which are components of the processing task.

\section{Task 4: Visualization}

In our project organization, visualization includes the massive effort of data management as well as the specific techniques for visualization. We have been able to utilize the $3 \mathrm{D}$ visualization capabilities of our software packages for interpretation of the data we have processed, and have put the processed (DFM) data volumes from a subcontractor into a 3D visualization package for easier interpretation.

\section{Technology Transfer}

Our web site is complete and has been updated regularly (http://www.geo.mtu.edu/spot). We have presented a number of oral presentation and posters at various professional society meetings and smaller forums. We have published one paper in The Leading Edge and one in Geophysics; a chapter in a book is in press; and several other papers are in various stages of preparation and review. A number of meetings with specific groups with interest in one or more of the data sets have been held. 


\section{Summary of Results}

\section{Reflections are not layer properties:}

We all know that reflections can, at their simplest, be considered to result from the convolution of a source wavelet with a reflection coefficient, but we often forget that if the overlying layer changes property, the character of the reflection will change. Inversion of seismic traces for acoustic impedance (or elastic impedance for offset volumes) is intended to remove this ambiguity, and can work extremely successfully. In fact, we have made use of trace inversion to deduce appropriate input parameters for Gassmann-based fluid substitution and forward modeling for time-lapse monitoring. Inversion, of course, is model-driven, in that the user must define which well-log data to honor, determine the limits to put on the possible range of results, and decide how to interpolate between well control. But, in the hands of a highly-trained and careful practitioner, we feel that inversion is one of the most important tools available to the geophysicist/interpreter.

\section{Point measurements should not be made off of seismic peaks or troughs:}

In areas of thin beds, the use of point-based attributes (such as amplitude) can be extremely misleading without careful calibration. For example, some data sets have been interpreted in terms of seismic amplitude measured at some time above or below a continuous reflector. The amplitude so measured may result from the vagaries of wavelet interference, or even just a thickening of an intervening bed, and can lead to measurements made at different points on the 'flank' of the reflected wavelet. The resulting attribute map may resemble a geologic feature, but that resemblance may be fortuitous, or caused by a geologic feature other than the one we think we are mapping.

\section{Frequency-domain and time-domain studies simply describe the waveshape:}

In some cases, it is easiest to look at frequency-based results (such as spectral decomposition or instantaneous frequency), and in some cases, it is easiest to look at time-domain results (such as amplitude, arc-length, or isochron differences). But we should always keep in mind that these are simply ways of analyzing one aspect or another of what is really a complicated (and complex) waveshape. We should use whatever tool or technique is most appropriate for the occasion, but it is really the waveshape that we are studying. Recently, techniques for classifying the waveshape, within temporal windows in the data volume, have become available. These are based on neural network approaches, and tend to lose physical significance unless used with care; modeling and model-driven classification systems are becoming more-widely available, helping the interpreter to maintain the link with physical properties of the reservoir.

\section{The convolutional model is not correct:}

We all know that the convolutional model is an approximation: it assumes that the seismic volume is constructed of zero-offset traces, with or without multiples and migration artifacts. But we also all know that amplitude-versus-offset is an important tool in pre-stack analysis of data. We often forget, unless reminded, that the final stacked seismic volume or section is a result of stacking data from various offsets, with their various amplitudes. The final stacked trace 
is not equivalent to a zero-offset trace. And yet, we tend to use it as such much more often than we should.

\section{The synthetic must tie:}

We should place a great emphasis on obtaining a synthetic seismogram that fits the recorded data extremely well, or we cannot place much confidence in our interpretation. This is not often easy; all the problems described above conspire to reduce the value of the synthetic seismogram. But if the synthetic does not tie the data, there must be a reason, and it is the task of the prudent geophysicist/interpreter to find the reason; it may be due to offset effects, to ignoring the effective theory model in upscaling of thin layers, or any number of other effects. The inversion process, by its nature and as implemented in most software packages, forces the user to extract from the data a wavelet that is representative of the wavelet that is (likely) present in the data (assuming the convolutional model, of course). This emphasis on wavelet extraction is probably one of the reasons that inversion is often so successful in aiding interpretation.

\section{Multiple attributes can help, or they can hinder:}

Much has been written recently on the likelihood of obtaining spurious correlations if, as is often the case, one can combine many dozens of attributes from a few wells in the search for a significant relationship. We expect to find, for example, one apparently significant correlation at the $95 \%$ confidence level from among 20 possibilities - from a RANDOM data set! In general, one should try not to use more than about three 'independent' attributes in combination.

\section{How constrained must we be? How detailed should our relationships be?}

The scientist in each of us wants to prove the calibration of each attribute beyond a doubt. The pragmatist in each of us wants to find a correlation and use it. We should all strive for an engineering efficiency, where we find the correlation, explain its origin to our satisfaction, and then use it to the advantage of our asset team. But we should always be looking for a possible pitfall. For example, we may find a relationship between a specific attribute and pay in an environment where a facies (say, a reef) is either present or absent. But the use of that relationship within a reef facies to determine the actual net pay would be unwise; in fact, the relationship may be exactly the opposite within the one facies, and our interpretation would be grossly in error. Occasionally, the quick answer is also the correct answer; but often, and perhaps most often, the quick answer is a poor answer.

\section{What level of rock-physics understanding is required?}

The simplest approach to understanding seismic attributes may often lead to misinterpretations. For example, many bright spots are due not to the zero-offset reflectivity, but to the far-offset reflectivity added through the stacking process. An interpretation in terms of acoustic impedance alone will be grossly misleading. Likewise, the effect of pressure changes on the rock dry-frame properties may be as important in a time-lapse monitoring experiment as the effect of pressure on the fluid phase and properties; often, these effects are in opposition, and ignoring one of them is done at great peril. 


\section{General Conclusions:}

The processing/interpreting geophysicist should approach the problem of extracting petrophysical information from seismic data (performing 'seismic petrophysical' analysis) from two directions, each with parallel processes. One is the processing of seismic data - this should be performed to bring the data as close as possible to true amplitudes, true capture of offset effects (often through creation of two volumes: the acoustic, or zero-offset volume; and the elastic, or far-offset volume), and, ultimately, as close as possible to true LAYER properties, through careful inversion. Remember, it is the layer property that interests us; it is a quirk of nature that provides us with reflections at interfaces instead. The second direction is the understanding of the rock physics involved, and the effect that various physical parameters (saturation, thickness, porosity, etc) can have on the seismic waveshape. We generally accomplish this through modeling, and the creation of synthetic seismograms for various scenarios, but often the attention is lacking at the detail required to truly understand the effects. In any given data set, there will typically be impediments to performing the calibration of seismic attributes in a thorough manner, and challenges to overcoming limitations in data availibility or quality require a knowledge of the geologic model, of rock physics, of logging, of seismic processing and acquisition, and of interpretation techniques.

\section{Field Studies}

We studied several fields in this project. The main field studies were at Stratton, Boonsville, Wamsutter, and Teal South. Additional studies were made at Crystal and Waha fields. These are discussed in the following sections.

\section{Crystal Field 2D seismic data, Michigan}

We made use of a 2D seismic line for which we had prestack (raw field record) seismic data, in order to evaluate the usefulness of such data for seismic attribute analysis, and to investigate the importance of seismic acquisition artifacts (the seismic acquisition 'footprint'). We also hoped to be able to actually use this line for attribute analysis over a field that had formed the basis of an earlier DOE-supported Class II project.

The seismic line had been acquired in 1986, and had been processed commercially for a goodquality poststack section, which had also been used in previous work by us. Even though the poor quality of some in-house reprocessing, without refraction statics, resulted in a line that could not be used with confidence for attribute analysis, it did provide us with an excellent case for the study of acquisition artifacts because the line was irregularly sampled and because the incomplete statics could be considered as an additional acquisition-related problem. Figure 1 shows some displays of the seismic data in conventional format.

The Devonian dolomite reservoir comprising Crystal Field occurs at an early time in the seismic data, and the fold at this time is low, generally about 6 after mute. The details of the mute are important, because the ground roll can easily be summed into the stacked trace in some CMP gathers, and not in others, greatly affecting the amplitude and other attributes of the stacked data. The use of vintage data, acquired to identify prospects at one depth (in this case, much deeper) and used to investigate a different depth, should be discouraged. If we followed this line of investigation any further, we certainly would have found attributes that were meaningless in terms of reservoir properties. 

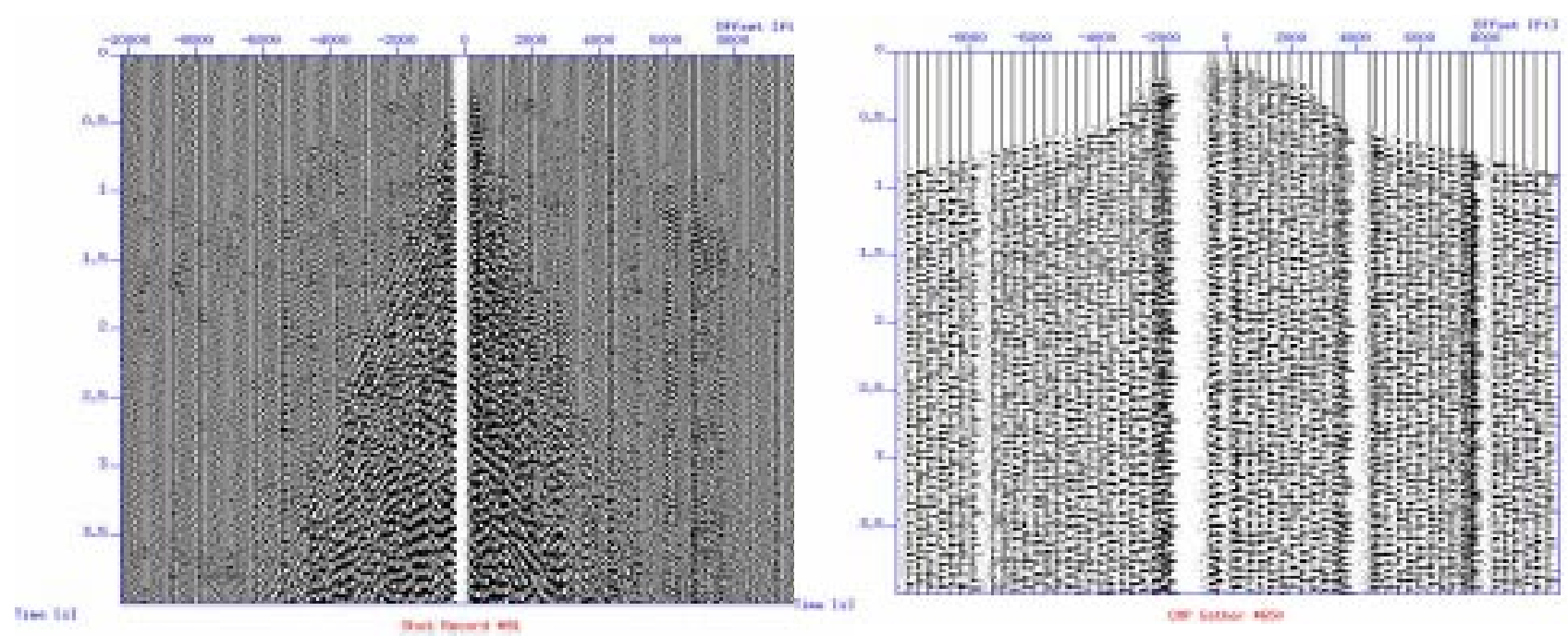

Figure 1: Left side: A shot gather of the Crystal field data; Right side: a CMP gather.

We then turned to investigation of $3 \mathrm{D}$ visualization techniques for evaluation of $2 \mathrm{D}$ prestack data, by using rewriting the data as a 3D volume, with offset as the second horizontal dimension (CMP location is the first horizontal dimension, and time is the vertical dimension). In this way, we can quickly scan down the data (as a series of time slices) and across the data (in CMP gathers, shot gathers, or receiver gathers) and identify artifacts or anomalies of interest. Figure 2 shows one such set of displays in a perspective view.

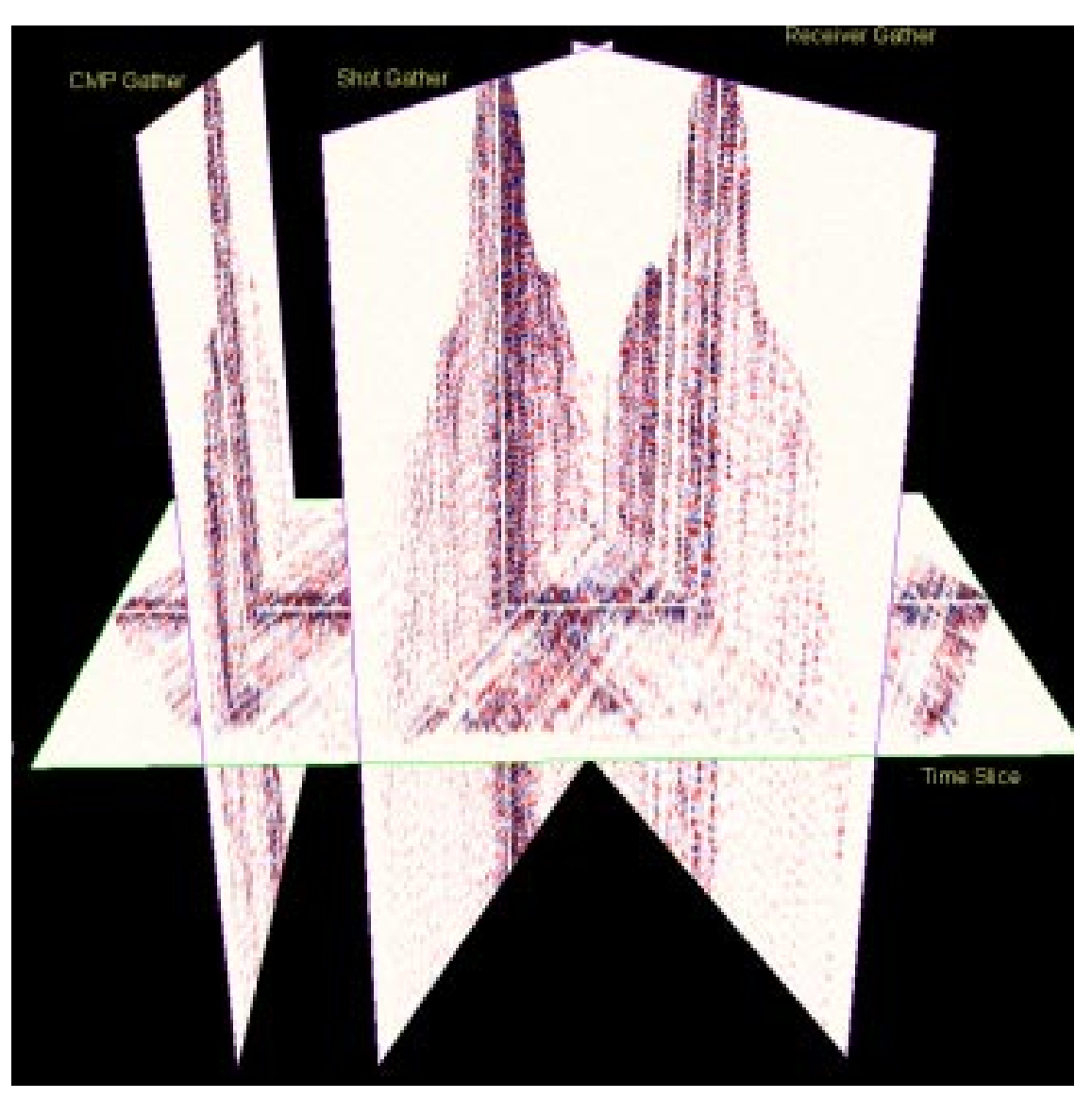

Figure 2: $3 D$ view of a prestack 2-D line. One shot gather, one common-receiver gather, and one common-midpoint gather are shown along with one horizontal timeslice. The influence of acquisition-related artifacts is readily apparent, in the striping and banding of amplitudes, as indicated by the colors. This data were NMO-corrected and muted prior to display.

We have used this (Crystal Field) data set to test various techniques for recognizing and avoiding acquisition footprints, but did not pursue its analysis any further. 


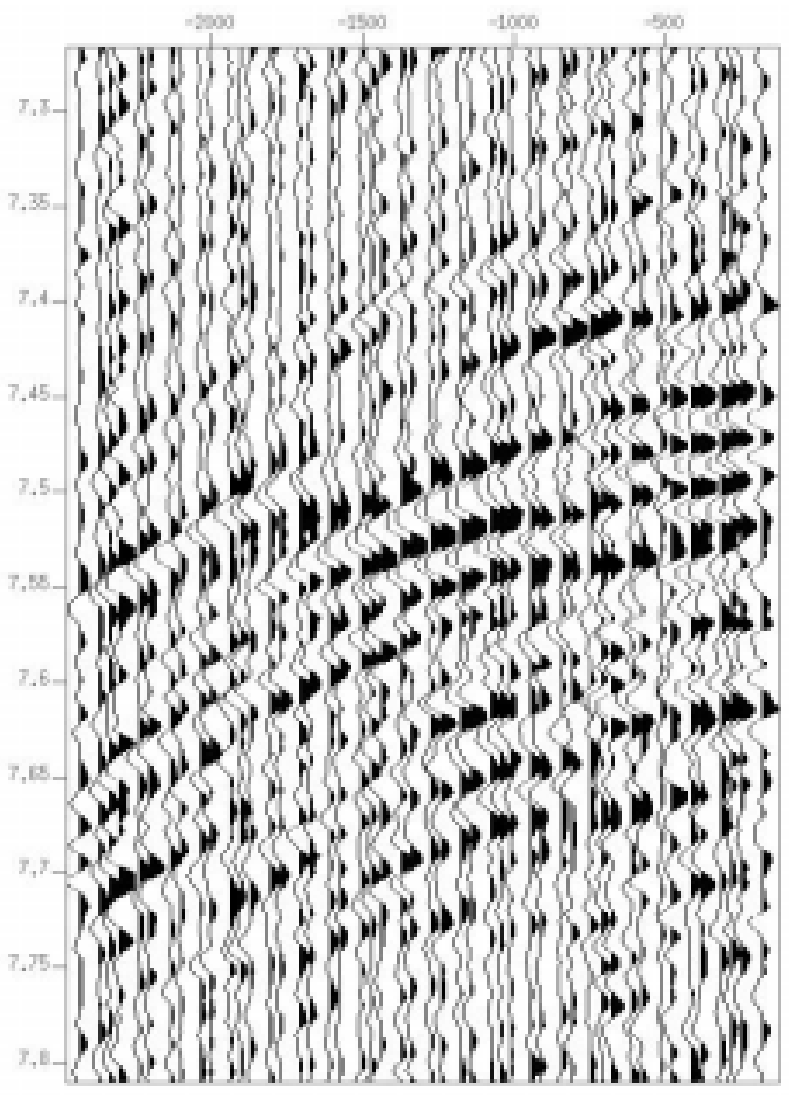

High-quality data from a different 2D line shows that the usual assumption that stacked data is equivalent, in principle, to a zero-offset seismic trace is invalid. Figure 3 shows a typical 2D shot gather, demonstrating this.

Figure 3: Seismic gather from the Pacific

Ocean.

\section{Stratton Field, South Texas}

The public-domain data set from Stratton field provided the challenge of imaging features below seismic resolution, in an effort to identify the channels known to be present in the volume. Some of the channels are readily seen, particularly at shallow levels, but most channels can only be inferred by correlation with well data and through recognition of subtle features on the seismic data.

The Stratton field produces from the Frio formation from reservoirs contained within a series of meandering channel-fill deposits. The Texas Bureau of Economic Geology (BEG), in a project supported by the U. S. Dept. of Energy (DOE) and the Gas Research Institute, studied this field in some detail (e.g., Hardage et al., 1994), in an effort to use seismic data to aid in identifying sources of reservoir compartmentalization. They also made a subset of the data available for public use. In their study, the BEG found that seismic data could resolve very small channels at considerable depth: down to $10 \mathrm{ft}(3 \mathrm{~m})$ thick, $200 \mathrm{ft}(61 \mathrm{~m})$ wide, at depths greater than $6000 \mathrm{ft}$ $(1800 \mathrm{~m})$. They interpreted some channels in the time structures (assuming that they represent paleo-topographic surfaces along which the streams flowed). They also observed some crosscutting amplitude features at some reservoir levels, which they presumed to be due to "residual effects from the deeper Vicksburg faults" (p.1655, in reference to reservoir level F39).

The data set is rich in thin-bed effects which change with depth to target. In a shallow section, above the depth range of hydrocarbon production, there are some small distinct channel features that can be readily identified, since the thin-bed tuning is less of a problem at these shallow depths. But identifying a channel is not sufficient, as the following discussion shows. 

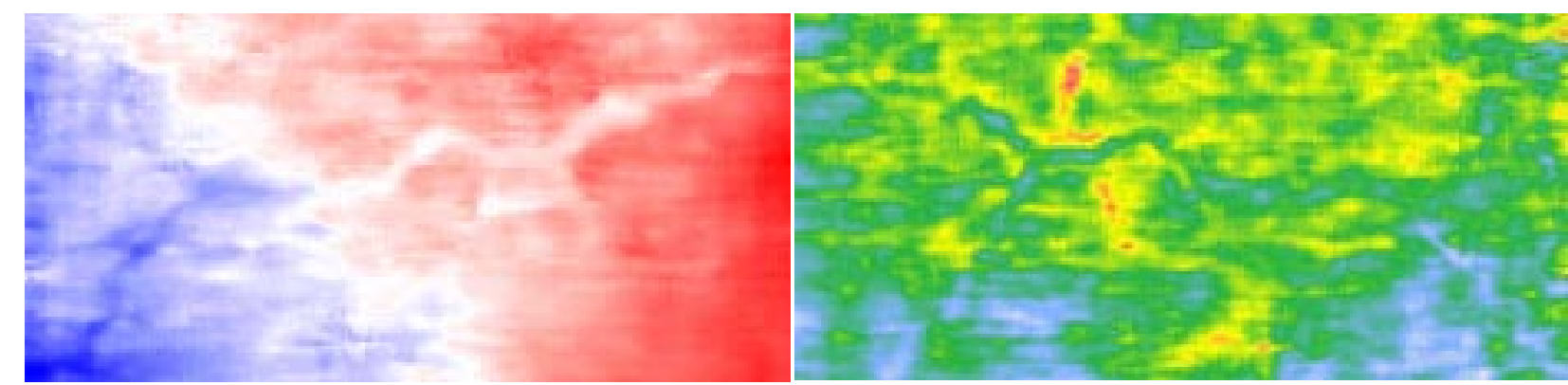

Figure 4: Shallow horizon in Stratton data showing apparent stream channels.

The left figure is colored by time structure (deeper times are blue), and the right figure is colored by amplitude. The amplitude attribute is clearly indicating the presence of some feature not apparent on the time structure, and that also appears to have the morphology of a stream. See Figure 5 for a threedimensional view of this paradox.

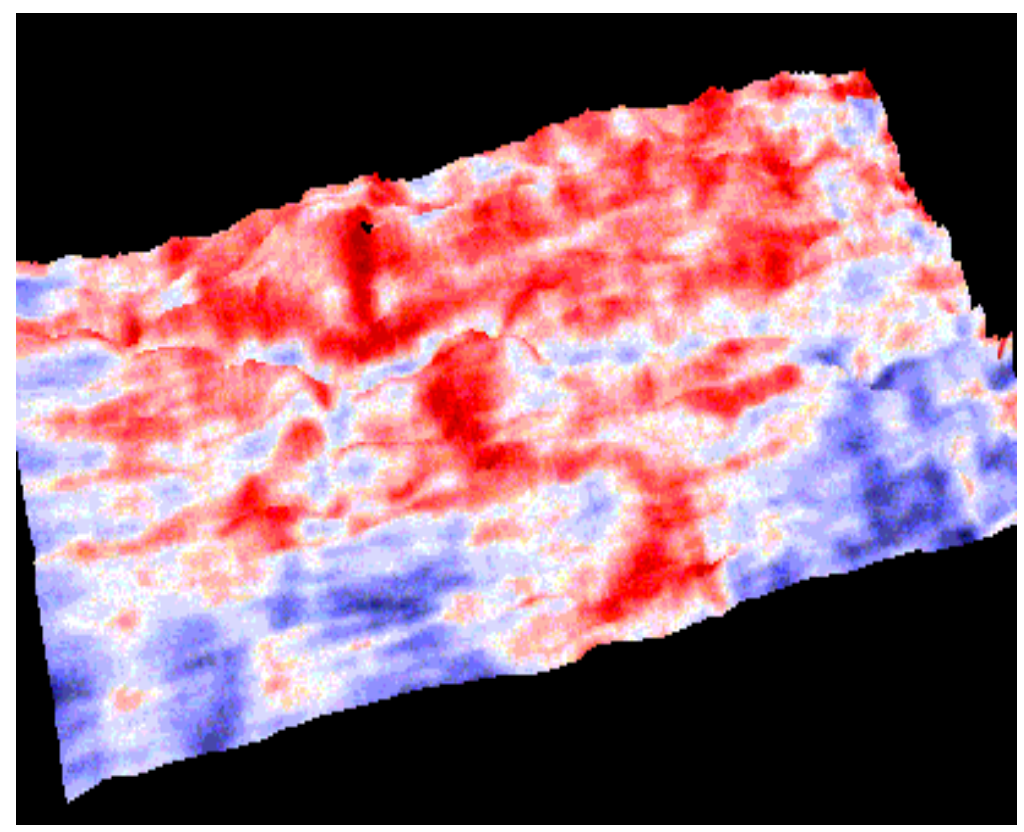

Figure 5: $3 D$ view of the shallow channel horizon.

In this image, the $3 D$ view has been rotated to provide a perspective view of the time structure, and the channel can be seen as a local depression or trough trending from the upper left $(N W)$ corner to the lower right (SE) corner of the image. The amplitudes on this time structure provide the coloring, and another feature, with the morphology of a channel but without an expression on the time structure, can be seen as a red sinuous body trending roughly $N-S$.

Conflicting evidence, shown in Figures 4 and 5, consists of two apparent channels, each visible by one attribute or another, along the same horizon. The time structure displays a channel that seems to trend NW-SE, while the amplitude display on that same horizon shows another apparent channel, running more or less N-S.

The Stratton field produces from the Frio formation from reservoirs contained within a series of meandering channel-fill deposits. The Texas Bureau of Economic Geology (BEG), in a project supported by the Dept. of Energy and the Gas Research Institute, studied this field in some detail(e.g., Hardage et al, 1994), in an effort to use seismic data to aid in identifying sources of reservoir compartmentalization. They also have made a subset of the data available for public use. In their study, they found that seismic data could resolve very small channels at considerable depth, down to $10 \mathrm{ft}(3 \mathrm{~m})$ thick channels, $200 \mathrm{ft}(61 \mathrm{~m})$ in width at depths greater than $6000 \mathrm{ft}(1800 \mathrm{~m})$. They interpreted some channels in the time structures (assuming that they represent paleo-topographic surfaces along which the streams flowed), and also observed some cross-cutting amplitude features at some reservoir levels, which they presumed to be due to 
"residual effects from the deeper Vicksburg faults" (p.1655, in reference to reservoir level F39). The cross-cutting amplitude anomaly shown in Figures 3 and 4 of this report is in a very shallow horizon, and does not have an obvious relationship to features in strata immediately overlying or underlying it, bringing any presumed direct association with deep structure into question.

The BEG study used high-quality Vertical Seismic Profiling to provide very accurate well ties between the seismic data and the productive intervals. They then used those well ties to determine what part of a wavelet corresponded to the thin-bed reservoir interval. They tracked a horizon that was close to the reservoir interval, then extracted attributes for the interval that corresponded to the reservoir location; for example, they may have tracked a geophysically recognizable horizon that occurred at, say, $1586 \mathrm{~ms}$ at the well location, and then extracted the amplitude at the time that a reservoir interval occurred, say, $42 \mathrm{~ms}$ above that horizon. That extraction rarely occurred at a peak or trough in the seismic data, but at some other location within the wavelet. This approach is pictured schematically in Figure 6. In addition, the details of event-picking constraints can determine some subtle differences in the event location enough difference to provide different attribute values for the same "event", as shown in figure $7 .$.

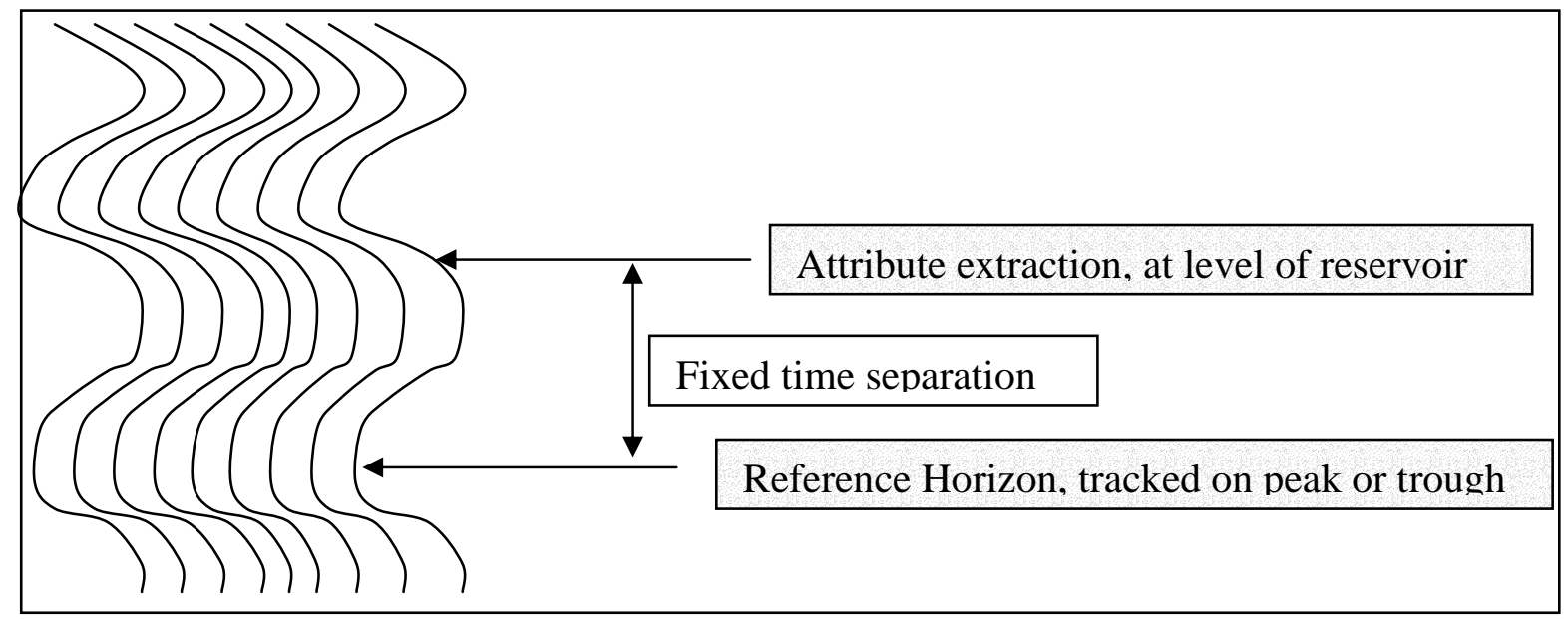

Figure 6: Schematic diagram summarizing approach used by the Texas BEG in Stratton field.
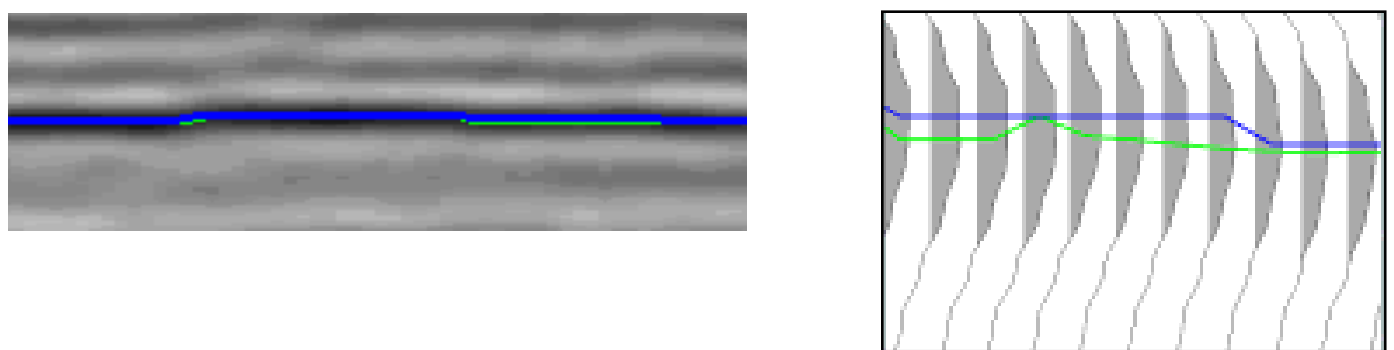

Figure 7: Stratton data picked with slightly differing constraints (in blue and green), using nearest peak or autocorrelation maximum (left: general view; right: close-up view). 
We have tried to duplicate this approach used in the BEG study, with mixed success, probably due to the limited size of the public-domain data set, compared with the larger volume of data with which the BEG based their work.

We have extended this approach to include a complete wave-shape matching routine. The BEG approach of creating a second horizon at a given time-separation from an easily tracked horizon is now straightforward, and readily accomplished on most modern workstation software packages. Additional newer techniques have become available to classify the seismic wavelets themselves into different categories, or 'facies,' depending on details of their shape. The technique we have applied uses a neural-network approach to divide the wave-shapes found in the interval being considered into a (user-specified) number of different 'facies.' The maps displaying the spatial locations of the different facies can often provide a picture of geologically meaningful features, such as channels or crevasse-splays. We pursued this approach because the BEG approach indicates that subtle differences in wave character provide strong clues to the location of channels or to the compartmentalization of the reservoirs. However, just as simple attributes can be affected by details of the tracking method used, facies classifications can also be affected, as shown in figure 8.
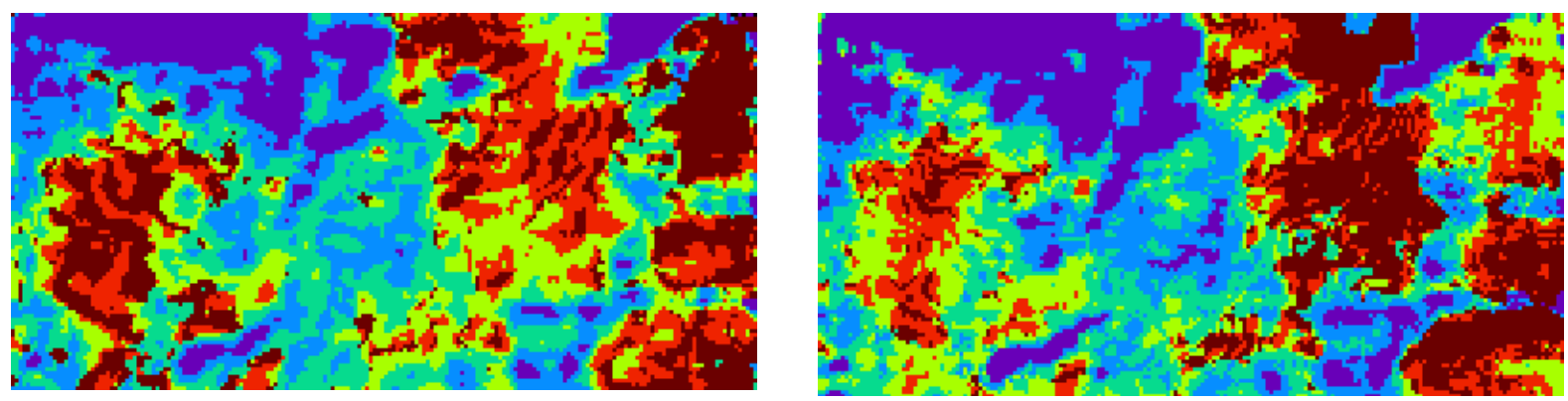

Figure 8: A comparison of facies classification based on the differently-picked horizons.

Figures 9, 10, and 11 show examples of the output from this approach, using a shallow channel (the same horizon as shown in Figures 4 and 5) for investigation purposes. Unfortunately, none of the wells penetrate this feature, and we are presently not sure what lithologic properties are providing this seismic response.

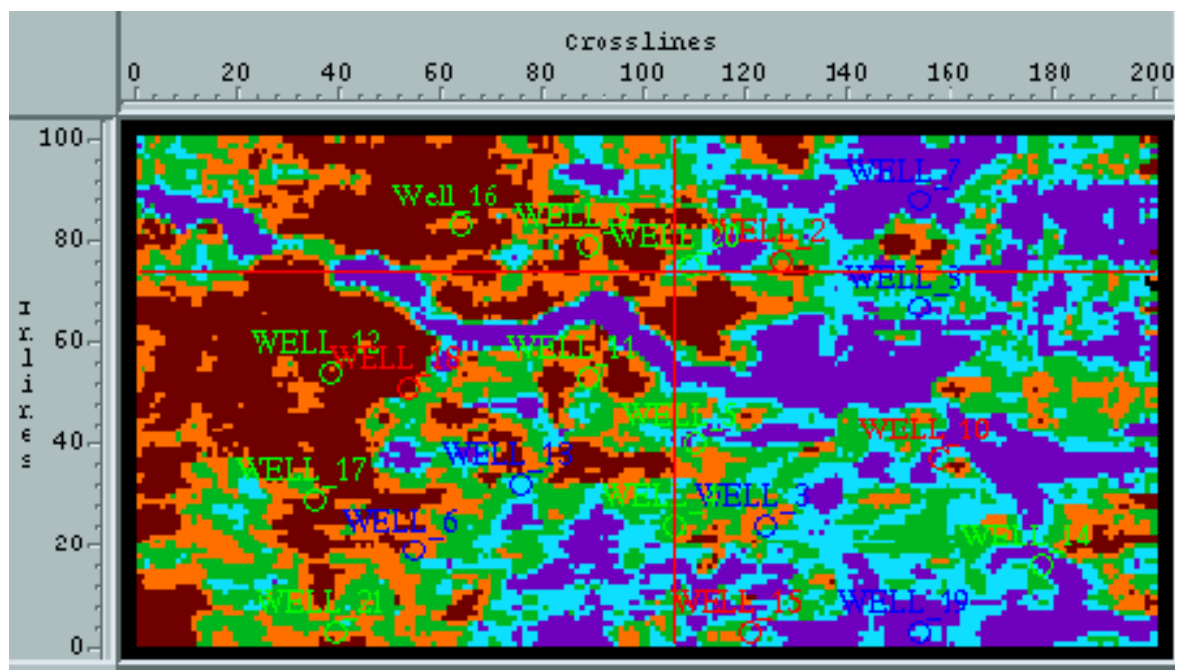

Final Technical Report

October, 2002
Figure 9: Seismic facies classification map of an interval near $840 \mathrm{~ms}$ in the Stratton data set. 


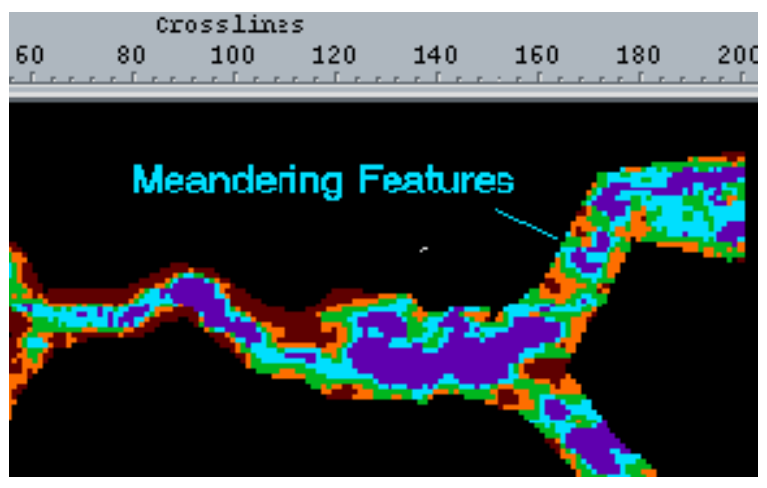

Figure 10: Cut-out region of channel, with detailed reclassification of facies within this channel.

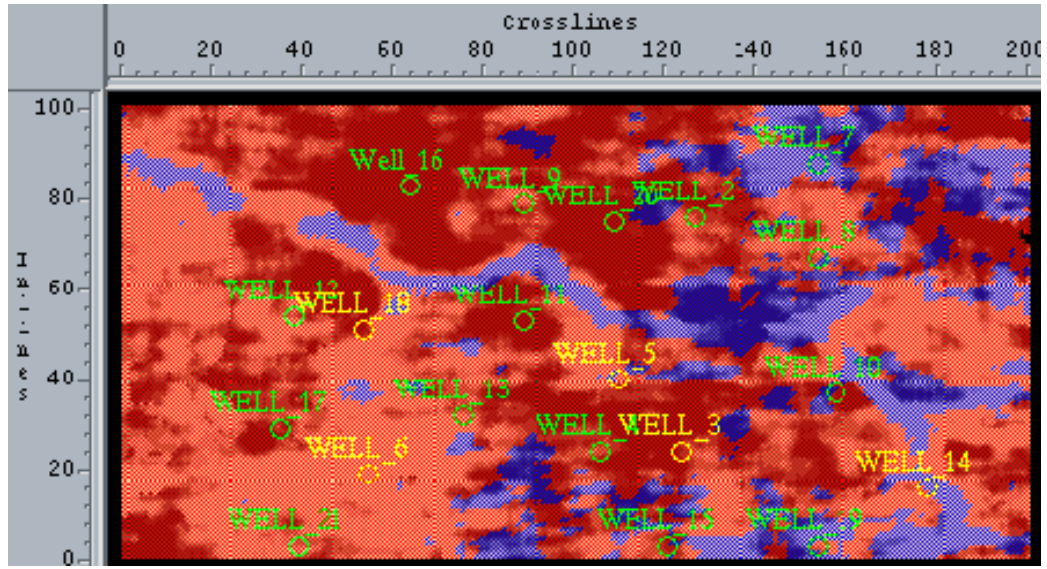

Figure 11: A combination of time structure and facies classification. in the Stratton data set.

In their study, the BEG found that seismic data could resolve very small channels at considerable depth: down to $10 \mathrm{ft}(3 \mathrm{~m})$ thick, $200 \mathrm{ft}(61 \mathrm{~m})$ wide, at depths greater than $6000 \mathrm{ft}(1800 \mathrm{~m})$. They interpreted some channels in the time structures (assuming that they represent paleotopographic surfaces along which the streams flowed). They were generally unable to 'track' the specific horizon of interest in this thin-bedded environment, partly because not all horizons were continuous, and partly because the geologic bed of interest (as indicated in well logs) did not correspond to a clear peak or trough. They made extensive use of 'phantom' horizons (our word, not theirs, but now in common usage), at a constant offset from readily-tracked horizons, and tied at one well where a VSP was conducted.

Our observations and maps initially were similar to those obtained by the earlier studies conducted by the BEG. However, our interpretation of the physical origin of the features on these maps is significantly different from theirs. We have concluded that most of the features recognized from these phantom horizons are in fact due to isopach differences between the phantom horizon and the local horizon; these may, in turn, be due to the presence of channels (in one case this is clear). But the attributes themselves are not directly associated with lateral changes in horizon properties - instead, the 'phantom' horizon crosses true horizons as the interbed thickness varies, and the determined attributes naturally change as these beds are crossed. The details are presented in the following summary. 


\section{BACKGROUND}

The Stratton field is located in South Texas approximately 30 miles southwest of Corpus Christi (Figure 12) in Kleberg and Nueces counties. The 2 square-mile migrated 3-D seismic volume, containing 100 inlines and 200 crosslines spaced at 55 feet intervals, sampled at $2 \mathrm{~ms}$ in time, has been provided by the Texas Bureau of Economic Geology (Hardage et al, 1994) together with data from 21 well $\operatorname{logs}$ and one Vertical Seismic Profile (VSP). The data were acquired using an 8-120 Hz Vibroseis sweep. Like many gas fields with a long production history, the log suites vary greatly among the wells. The wells were drilled from the 1950's to the early 1990's and generally were logged with gamma, resistivity, and neutron logs. Since sonic logs are not available for any wells in the field, we rely on a checkshot derived from the VSP. There are no log data available for the nonproducing shallow channel region investigated here. We have received limited production data provided by the operator Collins \& Ware.

The major producing formations are the nonmarine fluvial Upper Vicksburg and Middle Frio formations (Figure 13). The Upper and Middle Frio contain nonmarine channels; the Lower Frio has been described as a coastal plain, and the Upper Vicksburg Formation is thought to be deltaic in origin. The Frio formation is comprised of thin-bedded sandstones and mudstones as thin as $10 \mathrm{ft}$. A series of growth faults in the Vicksburg terminate at an unconformity at the base of the Frio. The horizons and growth faults are identified on a seismic section in Figure 14.

In this study, we analyzed a shallow non-productive horizon containing an apparent channel, and a deeper productive horizon just above the Frio-Vicksburg unconformity.

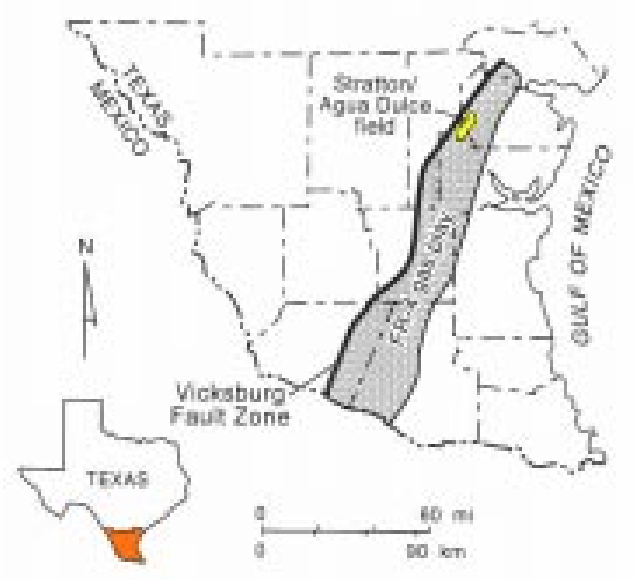

Figure 12 Location of the Stratton Field (adapted from Hardage 1995).

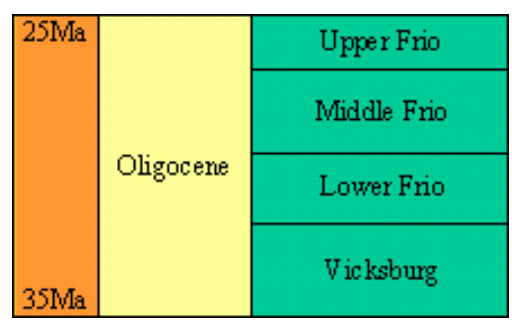

Figure 13 Stratigraphic sequence of the Oligocene 


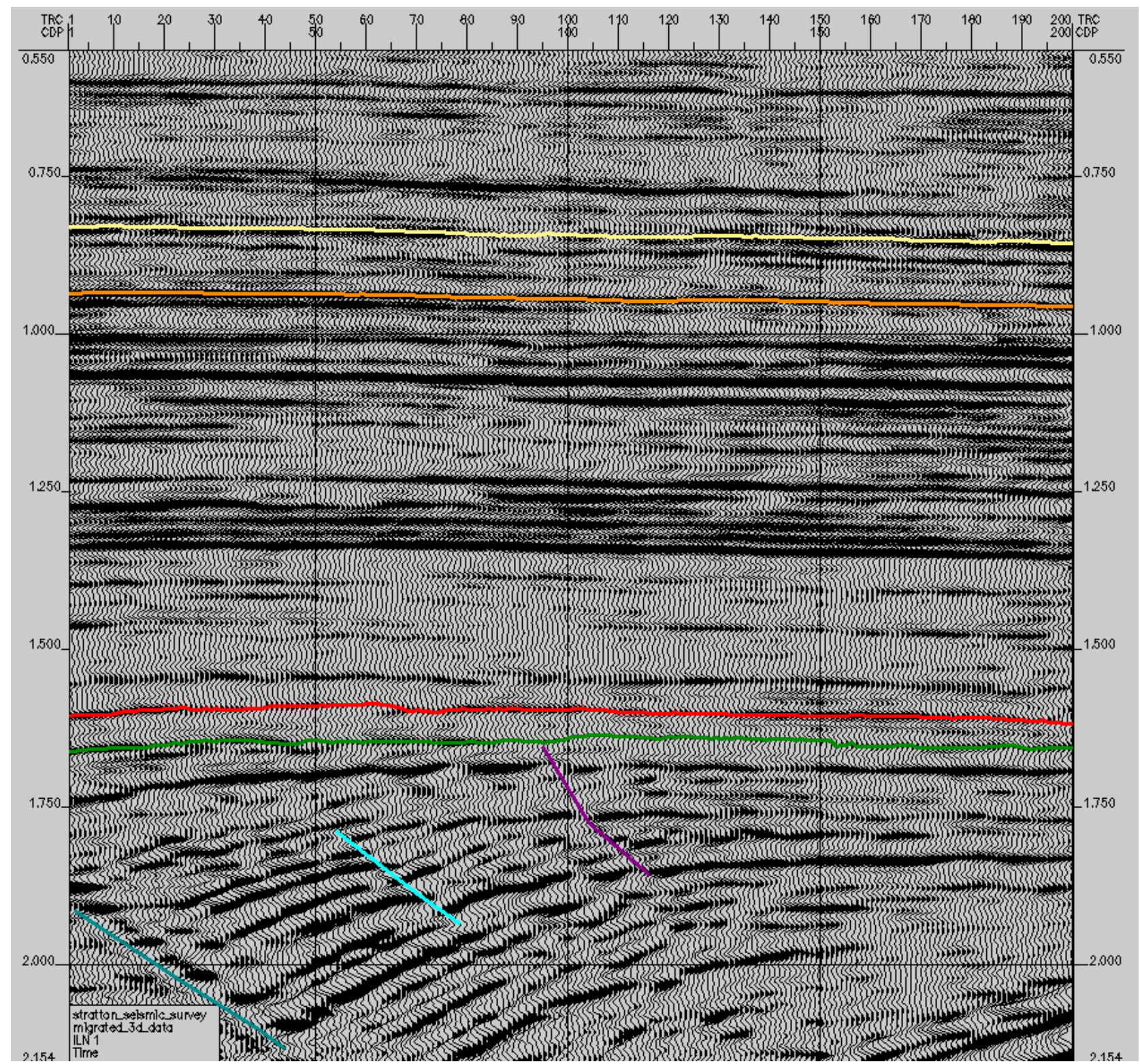

Figure 14 Seismic section showing the two horizons studied: a channel region at approximately $842 \mathrm{~ms}$ and the primary producing region at approximately $1640 \mathrm{~ms}$ in time. The heavily faulted area is due to a series of growth faults in the Vicksburg formation.

\section{PHANTOM HORIZON TECHNIQUE}

Many of the horizons of the Stratton Field were difficult to track across the entire survey. To address this problem, we followed a suggested procedure outlined by Hardage et al (1994) adapted to modern workstations. A shallow channel region and a deeper producing region were chosen as test cases (identified on Figure 14). A reference horizon was chosen close to each horizon of interest and tracked throughout the survey. Using the VSP data provided, each horizon was associated with a specific depth and the time differences between the horizons noted at that well. Using this fixed time difference between each reference horizon and its associated 
target depth/time, a new 'phantom' horizon is created. [This process is equivalent to flattening on a horizon and selecting a time slice relative to it.]

\section{Conventional attributes}

To better image the shallow channel and deeper producing horizons, conventional attributes were derived. Conventional attributes can be divided into point based and interval based attributes. Point based attributes include, but are not limited to, amplitude, instantaneous amplitude, instantaneous phase, and instantaneous frequency. These attributes are derived directly on a horizon. Interval attributes involve using a time window based upon an interval of a horizon or an interval between a horizon. Calculations that involve averages over a given time window, such as RMS amplitude, are examples of interval based attributes.

\section{Facies classification technique}

The term "seismic facies classification" can have many different meanings. For the purposes of this study, seismic facies classification is used to describe the process of grouping the traces of a survey into a limited number of categories based on the shape of each trace over a fixed time window (Poupon et al, 1999). To perform a seismic facies classification, a horizon and a fixed time window surrounding the horizon of interest should be chosen and the amplitudes extracted with the associated times. For example, if a $12 \mathrm{~ms}$ interval is chosen, with a $2 \mathrm{~ms}$ sample rate, there will be 7 amplitude values at 0,2,4,6,8,10 and $12 \mathrm{~ms}$ in time within the window. These samples are used to compute gradients by calculating the change of the value between samples (Figure 15). For this example, a list of 6 gradient values would be created. The series is then normalized by dividing each value by the largest gradient value. The series can be thought of as a 6 dimensional vector with each dimension having a value ranging between minus one and one. These vectors are then obtained for every trace in the survey or area of interest. Next, a neural network process searches for clusters of these vectors, a process roughly equivalent to finding clusters of waveshapes.

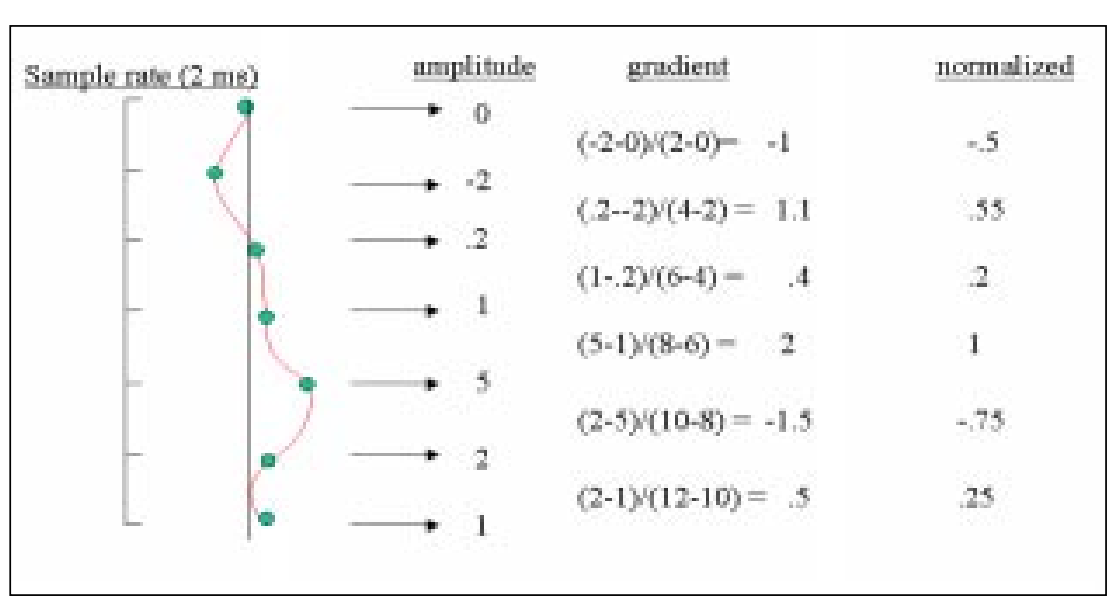

Figure 15: Calculating normalized trace gradients for the facies classification.

Each trace is then placed into a category or class, based on these clusters. Each trace has been classified based on its normalized gradient vector, and it is primarily the trace shape (changes in amplitude) that drives the

classification rather than direct amplitude values. The number of classifications will determine the resolution of the trace shape analysis. As more classes are used, smaller and smaller details will become visible in the analysis, which are shown as color changes in a facies classification map, but the noise in the image will also tend to increase. If more detail is needed, the survey can be subdivided into multiple regions to improve the classification process. A smaller region 
will have fewer traces and will need fewer classifications to provide the same amount of information.

The previous work performed by Hardage et al (1994) limited the analysis to point based amplitude observations. If a point based amplitude analysis can give information about a feature, then performing an analysis using the entire trace over a window of time can be expected to provide more details about that feature. Furthermore, if a single instance of time near a horizon can give useful information, using a window of time above and below that horizon should be able to give even more information. For example, if the producing region is present either slightly above or below a horizon, utilizing a time window that includes these areas may show features that might have been missed if the attributes were calculated directly on a horizon. Examining the trace shape over the time window could provide clues to the inherent waveshape property or properties that are associated with the fluid content. Using neural network technology, the traces (over a certain window of time) can quickly be divided and placed into a similar model trace, which can then be used to identify structural features. Classifying the shape of the trace should allow the delineation of more detailed features over a region than using a single point based attribute. The more classes used, the fewer the number of traces in each class, and the more detailed the analysis.

For the first example in the Stratton Field, a channel will be identified, and the facies classification technique will be used to later separate the traces based upon differences within the trace.

\section{SHALLOW CHANNEL REGION}

An apparent channel at approximately $842 \mathrm{~ms}$ can be located in the shallow region of the Stratton Field. This channel is easily identified from amplitude measurements on a time slice (Figure 16), but a phase change is introduced across this time slice by the gentle dip of the beds.

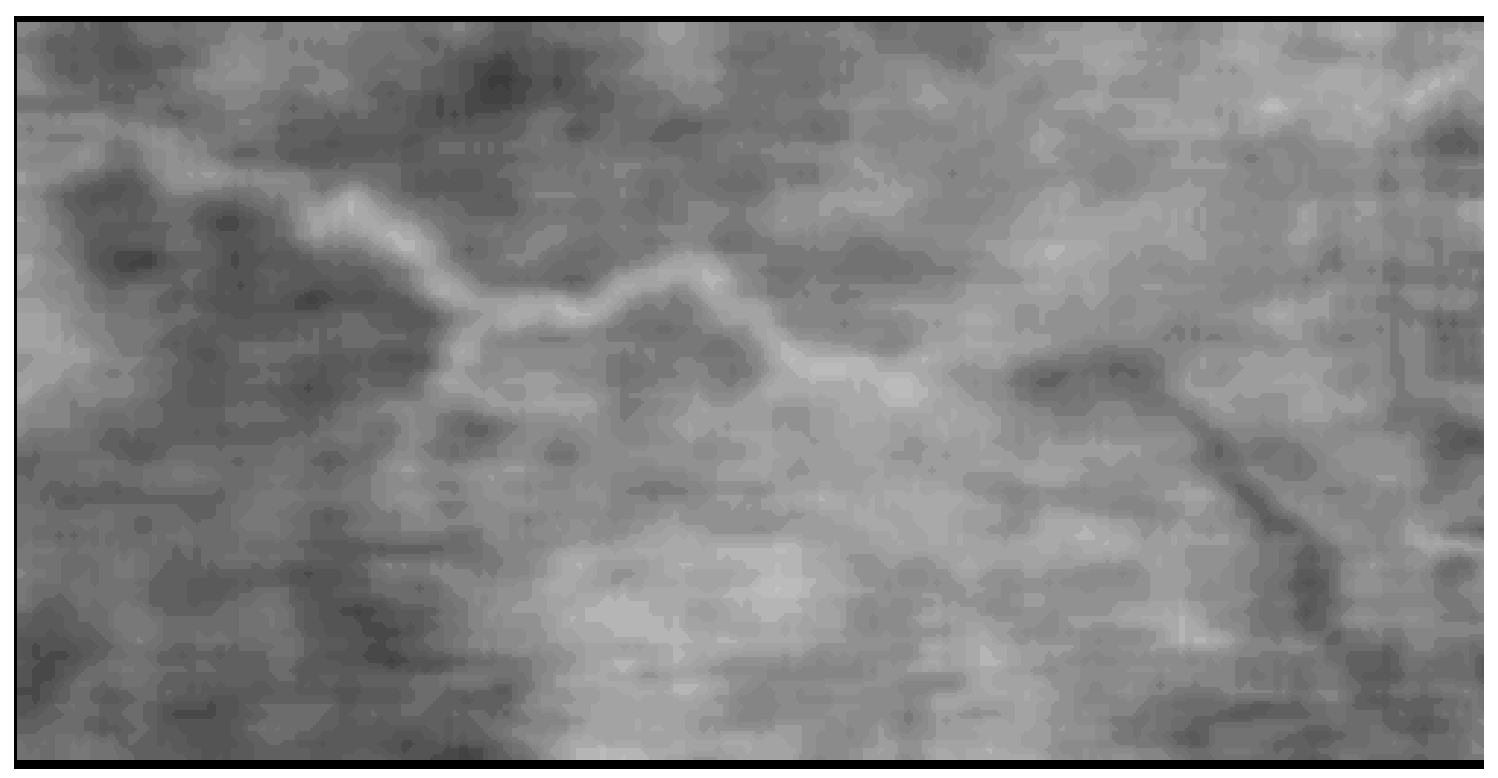

Figure 16: The shallow channel region at approximately $842 \mathrm{~ms}$ in time. A phase change occurs (from light to dark gray) across the image due to the gentle dip of the horizon. 


\section{Conventional Attributes on the shallow horizon}

Figure 17 shows the time structure of the interpreted horizon containing the shallow channel. Although visible, the channel is not clearly represented on this structural time surface, because the channel is consistently about $2 \mathrm{~ms}$ later than neighboring traces on the horizon, yet the horizon exhibits total relief (due to gentle dip) of about $40 \mathrm{~ms}$ obscuring the small time differences. Because the channel differs from the surrounding traces by only $2 \mathrm{~ms}$, the effect of the dip must be removed to emphasize these differences. A number of additional attributes were extracted along this horizon, none of which provided a more clear indication of the channel than the time structure.

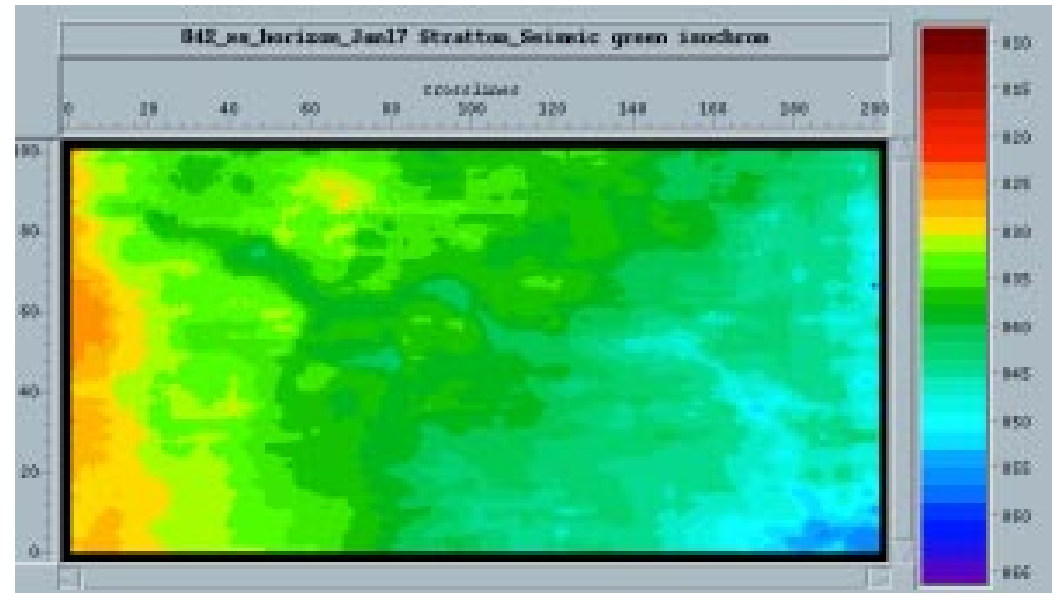

Figure 17: Structural (time) surface of the picked horizon outlining the channel.

\section{Conventional attributes using a phantom horizon}

A continuous horizon (found at $945 \mathrm{~ms}$ at well 9) was chosen and named reference horizon A (Figure 18). The time difference between the interpreted shallow channel horizon and reference horizon A at well 9 is $101 \mathrm{~ms}$. The time difference was chosen to overly the non-channel facies over most of the survey area. The new phantom horizon was created with the intention of removing the general dip structure from the shallower horizon. Attributes were then calculated over this phantom horizon, some of which are shown in Figure 19. Both the amplitude slice and RMS amplitude (which was calculated over a $20 \mathrm{~ms}$ window of time centered on the phantom horizon) do an excellent job of defining the channel, largely because the phantom horizon gently accounts for the dip, while cutting across the channel feature.

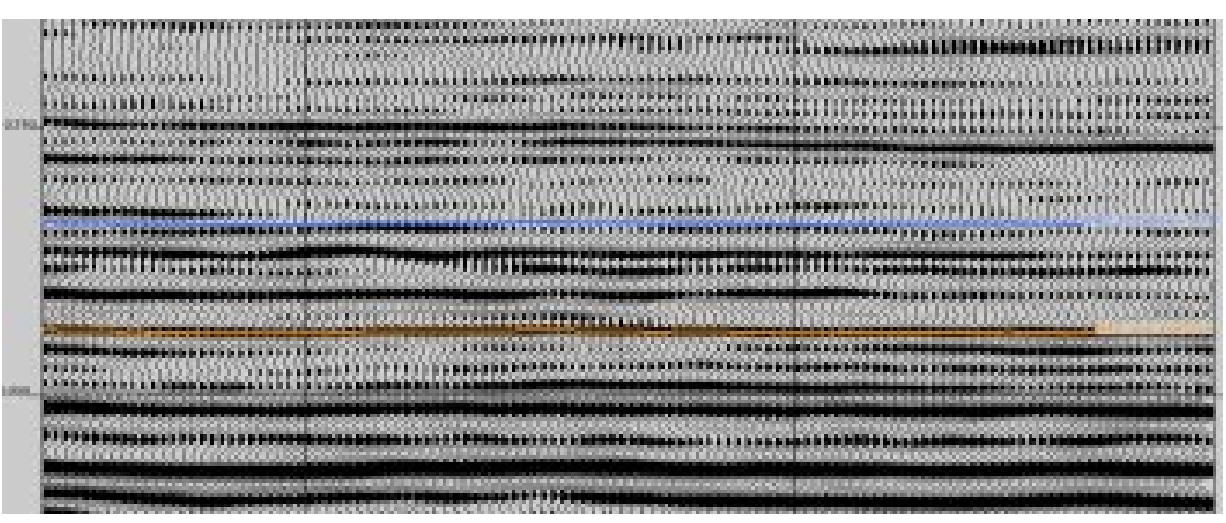

Final Technical Report

October, 2002
Figure 18: The phantom horizon created by subtracting $101 \mathrm{~ms}$ from reference horizon A. 


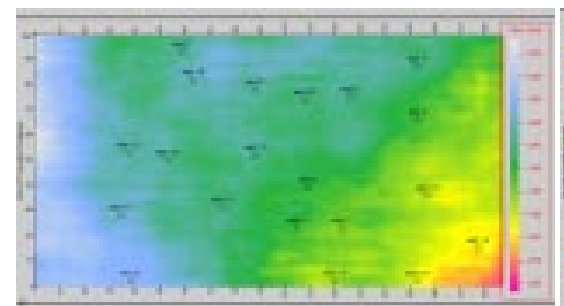

Left side: Time.

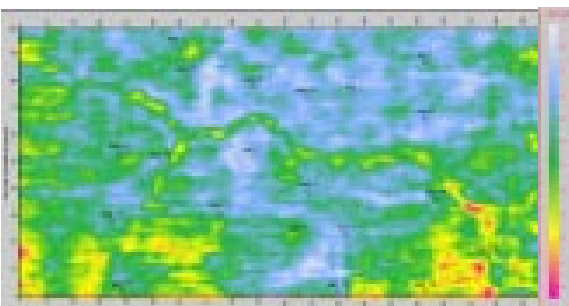

Center: Amplitude.

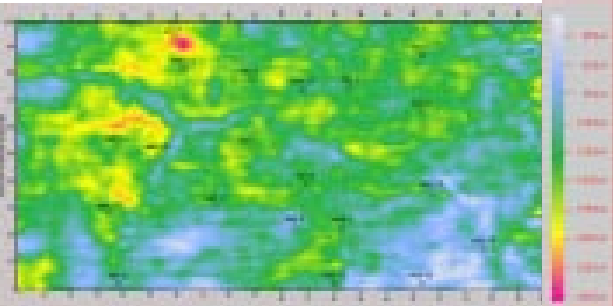

Right: RMS Amplitude

Figure 19: Attributes of the phantom horizon corresponding to the shallow channel.

\section{Facies Classification of shallow channel region}

The final approach applied to the channel horizon makes use of the facies classification technique. A $20 \mathrm{~ms}$ time window centered on the phantom horizon was chosen to perform the trace shape analysis. An unsupervised neural network with 14 classifications produced the image shown in Figure 20a.

The relatively clear boundaries emphasize the sinuous form of the channel. Figure 20b shows the model traces used to create the classification. Notice that a gradual time shift of the wavelets has occurred with approximately a $1 \mathrm{~ms}$ shift from one classification to the next. The channel is represented by the $13^{\text {th }}$ and $14^{\text {th }}$ classifications shown as dark blue and purple colors on the facies map. In effect, the trace shape of the channel has been classified by a small time delay; the facies classification technique is highly sensitive to time shifts.

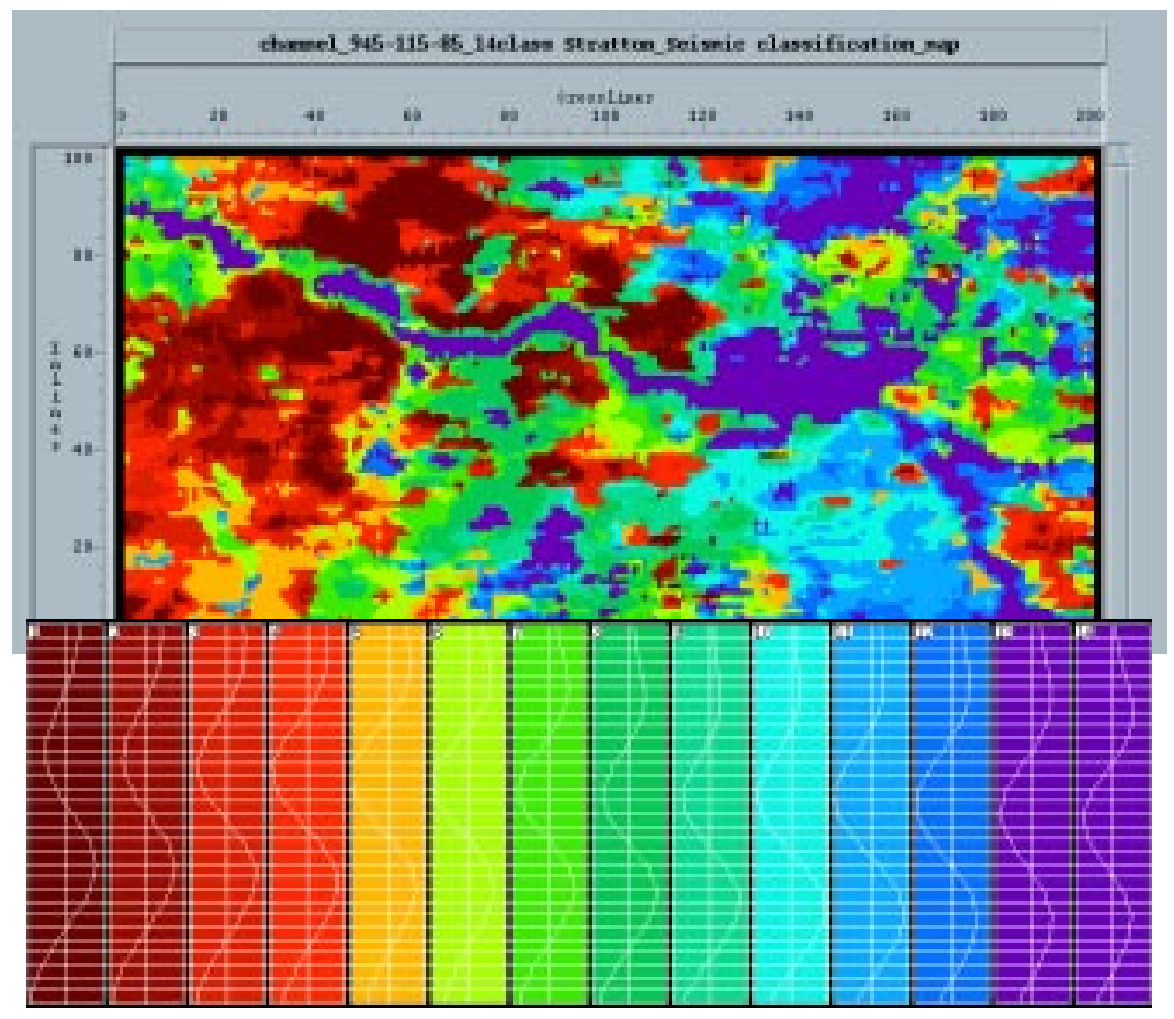

Final Technical Report

October, 2002
Figure 20: Unsupervised facies classification of the shallow channel region.

$20 a$ (upper figure) shows the classification, and figure $20 b$ (lower) shows the wavelets used in the classification. 


\section{Discussion of the shallow channel region}

In the shallow region, both point-based amplitude attributes and the interval attributes were not effective in imaging the channel when performed directly on the tracked horizon. The phantom horizon permitted much better identification of the channel than the original horizon, primarily due to the time shift involved. That is, because the channel reflection arrives about $2 \mathrm{~ms}$ later than the surrounding non-channel horizon, the phantom horizon encounters a slightly different phase at the location of the channel, and its amplitudes are correspondingly affected. The difference results in the dramatic color contrast between the feature and the surrounding survey. It is this color change that allows the viewer to separate the channel from its surroundings.

The seismic facies classification technique based on trace shape works for similar reasons. Each trace is classified as a multidimensional vector, with time shifts having a large impact upon the classification. The small time shift results in model traces created by the neural network that specifically image the channel. To image structures with small time changes, both phantom horizons and facies classification techniques based on trace shapes can be effective, but the user should recognize that the classification based on a phantom horizon may simply be due to isopach differences between the features being identified and the reference horizon.

\section{THE REgION OF Gas PROdUCtion}

For the second portion of the study of the Stratton field, we attempted to image a much more subtle case in the deeper region of production. Assorted well logs and limited production information are available for this region.

In the Stratton field, the F39 horizon is one of the primary producing intervals and appears on the seismic data at approximately $1640 \mathrm{~ms}$ in time. Unlike the channel horizon (tracked on minimum amplitude or trough), the F39 horizon was tracked using a maximum (peak) determined from times provided in Hardage et al (1994) at each of the well locations. Figure 21 shows the time structure of this horizon; we see a gently arching structure across the survey. As in the previous examples of the shallow region, amplitude, RMS amplitude, and instantaneous frequency were unable to decisively identify any channel or potentially productive zones. A phantom horizon was therefore created to better image this region.

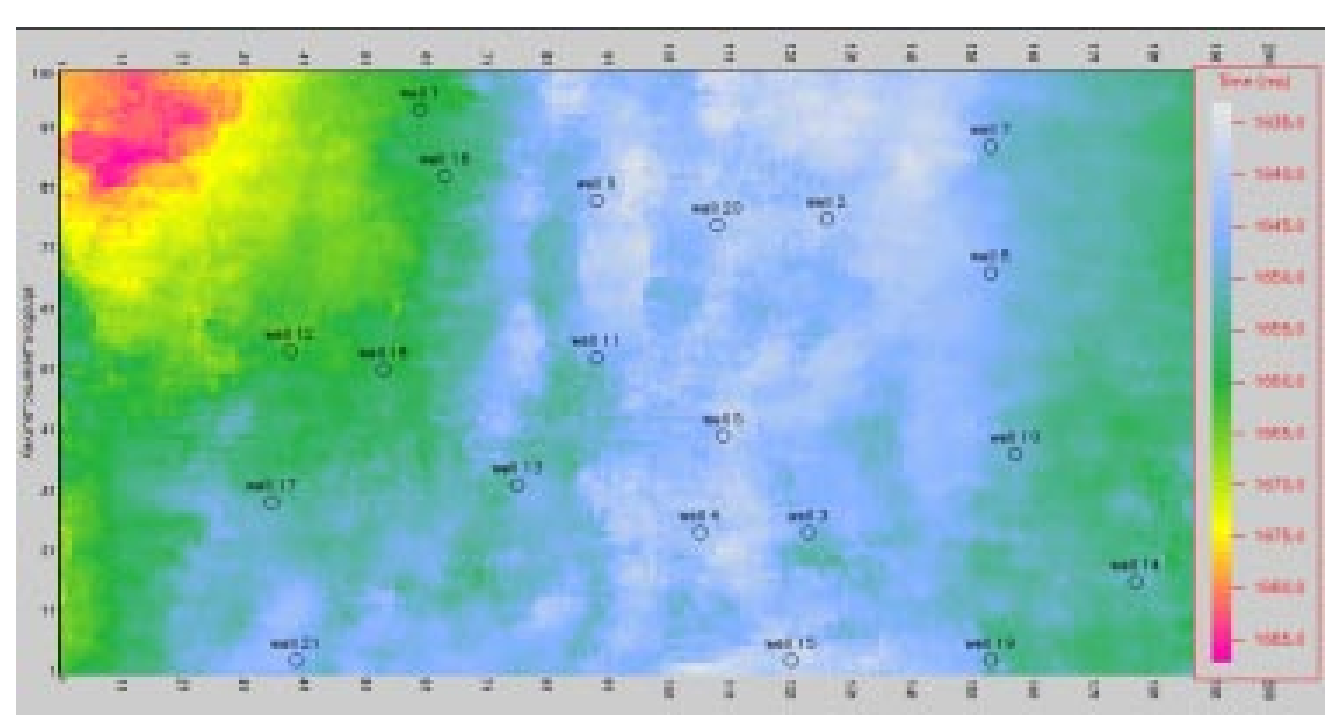

Figure 21: Time structure on the F39 horizon. 


\section{Conventional attributes using a phantom horizon}

The F39 horizon was difficult to track due to tuning effects. The phantom horizon technique used by Hardage et al (1994) was reproduced here: the F11 horizon was tracked and a phantom horizon was created at the level of the F39 horizon at the VSP well (59 ms later; see Figure 3, presented earlier). We then calculated attributes over this phantom horizon. Figure 22 shows the amplitude and RMS amplitude calculated on this phantom horizon. Examining these attributes show strong north-south linear features, and one may be tempted to conclude that these represent stratigraphic differences in the F39 horizon.

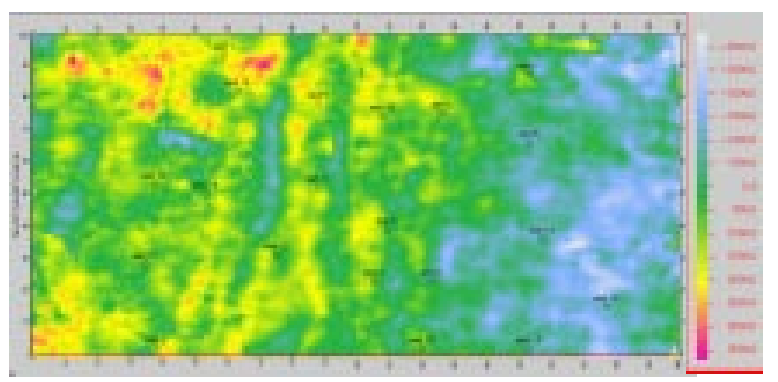

Left: Amplitude

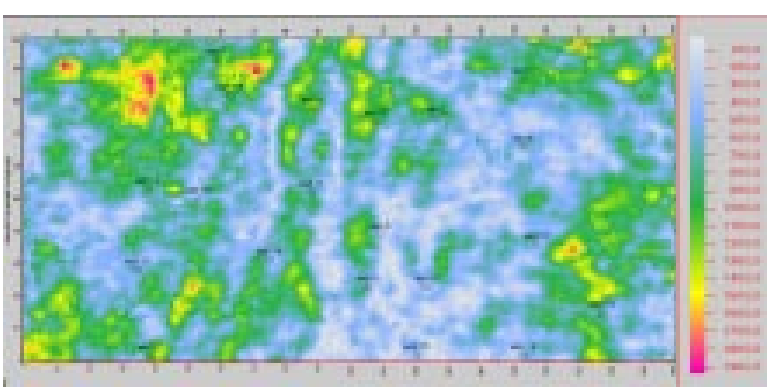

Right: RMS Amplitude

Figure 22: Attributes on the phantom horizon corresponding to the F39 level.

\section{Facies classification of the deeper producing region}

Using the Facies classification technique on the phantom horizon and a $25 \mathrm{~ms}$ time window (20 $\mathrm{ms}$ above and $5 \mathrm{~ms}$ below), we see a dramatic time shift in the classifications (Figure 23). Investigating these features more closely yields surprising results. These linear features are not

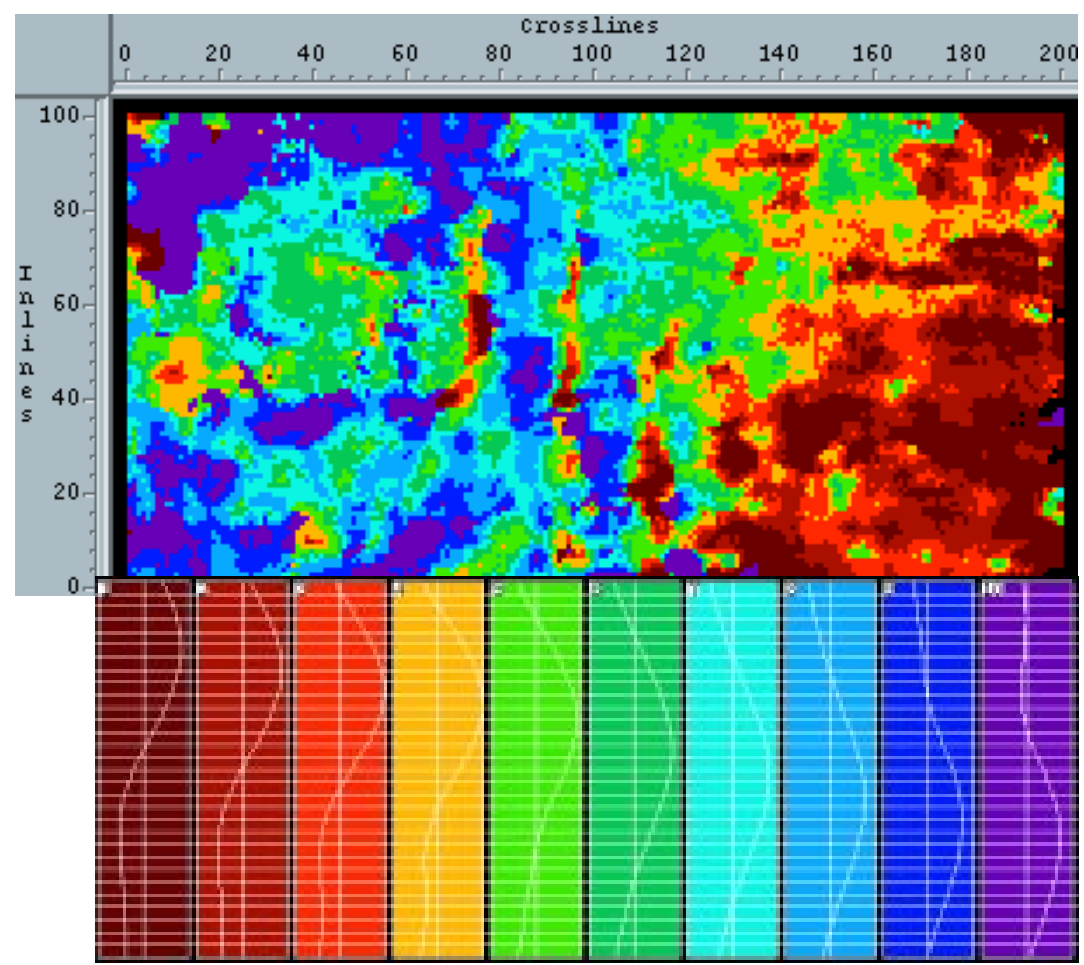
channel regions or areas of faulting as might be expected; they are areas (see Figure 24) where the true relief of the F39 horizon is much greater than that of the phantom horizon. The linear features are caused by the phantom horizon passing through alternating peaks and troughs.

Figure 23: Seismic facies classification of the F39 Phantom horizon. 


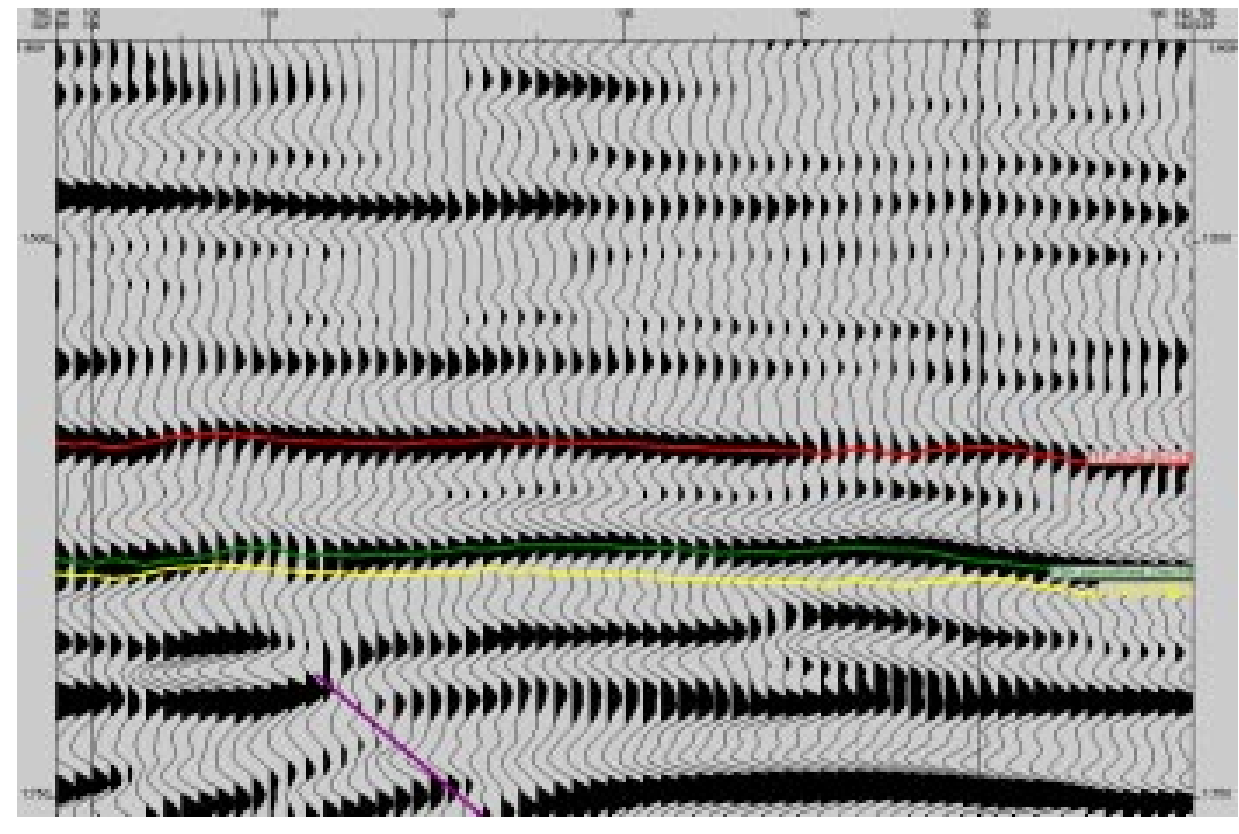

Figure 24: Horizons

associated with

tracking F39.

Red Horizon: F11,

used for reference

Green Horizon:

Tracked F39 horizon

Yellow Horizon:

Phantom F39 horizon, based on the F11

reference

To better portray the area between the F11 and tracked F39 horizons, an isopach map was created. Figure 25 shows that the isopach thickens to the west from $29 \mathrm{~ms}$ to $66 \mathrm{~ms}$ in time. These two horizons are not conformable and do not have the same dip; therefore, a phantom horizon based on a constant time difference is not advisable.

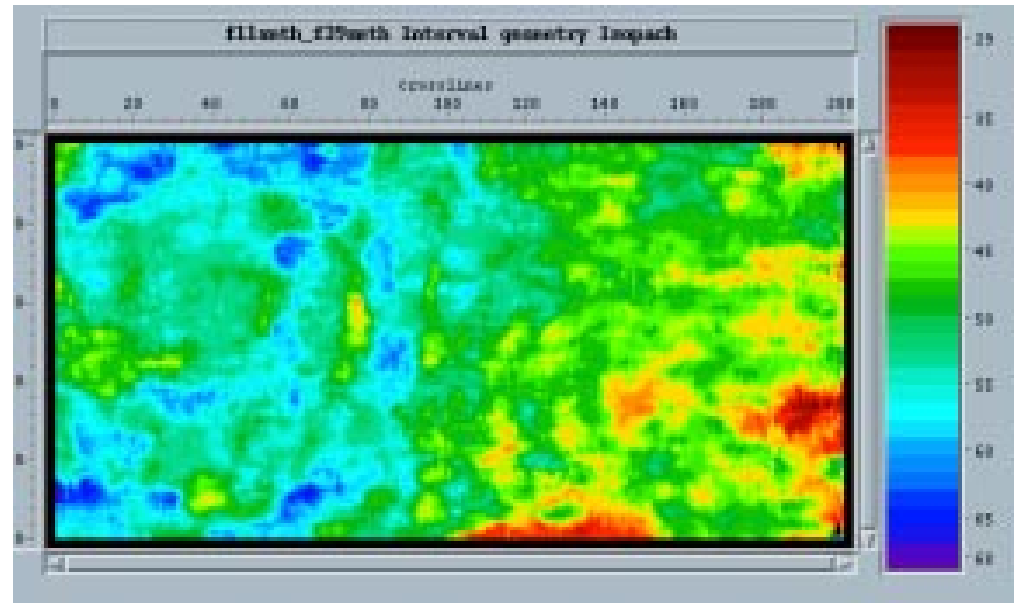

Figure 25: Isopach of time separation between the F11 horizon and the tracked F39 horizon.

\section{DISCUSSION}

To deal with the difficulties posed by non-conformable and/or dipping beds, we offer two suggestions. First, choose a reference horizon that is highly conformable to the horizon of interest. Most often this will be a horizon that is closely adjacent to the interpreted horizon. An isopach map showing the time differences between the reference horizon and the poorly tracked horizon of interest will aid in determining the degree to which the two horizons are conformable. In some cases, as in the F39 horizon, there are no nearby conformable horizons. Therefore, 
attributes must be calculated directly from the poorly-tracked horizon. We suggest using a waveshape-based facies classification scheme. As with the channel horizon, the gradually increasing time separation of the peaks is the driving force behind the classification. The trace shape analysis is very sensitive to time shifts caused by thickening or thinning of the nearby horizons. In the case of the deeper producing region, even anchoring on the horizon to be classified, resulted in a classification based on stratigraphic thickening.

Our second suggestion is to create proportional slices between the two horizons. This would work best for regions that do not have nearby conformable reference horizons. Two horizons, bracketing the poorly-tracked horizon of interest, can be used to create a set of proportionally interpolated horizons, which can be used, as phantom horizons that may be fairly conformable to the intermediate beds. These proportional slices will be less affected by conformable horizons since the medial slices will be used. Measuring amplitudes over these proportional slices may provide an alternative approach to horizon slicing.

\section{CONCLUSIONS BASED ON STRATTON FIELD STUdY}

The use of phantom horizons can provide strong assistance to the geophysical interpreter, particularly in areas where the level of interest does not correspond directly to a trackable event. The use of seismic facies classification schemes can provide additional strong assistance. But both approaches depend strongly on the phantom horizon's conformability with the horizon of interest. If the events are not conformable, attributes extracted along the phantom horizon will correlate more strongly with isopach differences than with true formation properties within a specific layer. On the other hand, if the interpreter recognizes these attributes as such, the phantom layer and its attributes can serve as a proxies for those geologic features that cause the thickening or thinning, but it is essential that the interpreter recognize them as such.

\section{DFM STUDY OF STRATTON FIELD}

The Stratton data set has also been analyzed by TransSeismic International (TSI), using their proprietary Dynamic Fluid Method (DFM). Their complete reports are provided an appendix to this report, and a brief overview of their technique is provided in a later section of this report, but some summary statements are made here concerning their observations in the Stratton field. The DFM technique uses instantaneous frequency and amplitude, removing the quickly-varying trends in time by averaging along horizons or time slices, and subtracting those averages. The remaining differences in frequency and amplitude are combined in a special algorithm, intended to be indicative of effective pressure (total pressure minus pore pressure). The areas of low pressure (or decreasing pressure, as this is considered to vary with geologic time, and is a dynamic phenomenon) are generally colored red in their displays, and indicate prospective zones for accumulation of hydrocarbons. In some environments, such as fractured tight sands, they expect to find accumulations, particularly of gas, along the boundaries between the regions of increasing and decreasing pressure.

The DFM interpretation of the Stratton field has indicated the presence of at least one large channel at about the $1250 \mathrm{~ms}$ interval; TSI identified this from a series of anomalies that they feel defines a stress concentration in the overlying and underlying strata from differential compaction occurred around the channel feature itself. They have concluded that the ancient streams have followed the edge of blocks present in the deeper Vicksburg strata, suggesting that movement on the Vicksburg faults continued at least through Frio time (and may continue even 
today). The summary figures supporting these interpretations are included here as Figures 26, 27,28 , and 29.

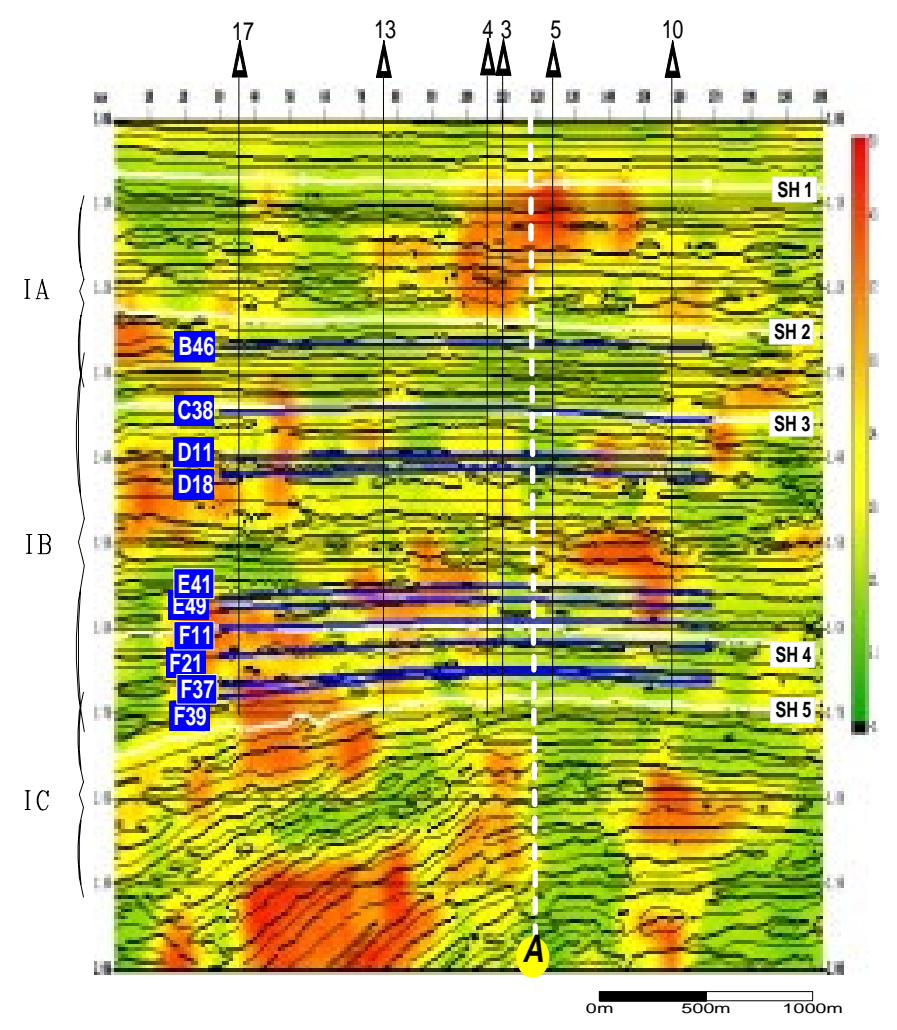

Figure 26: DFM results for Stratton field along one inline, showing regions of low effective pressure in red..

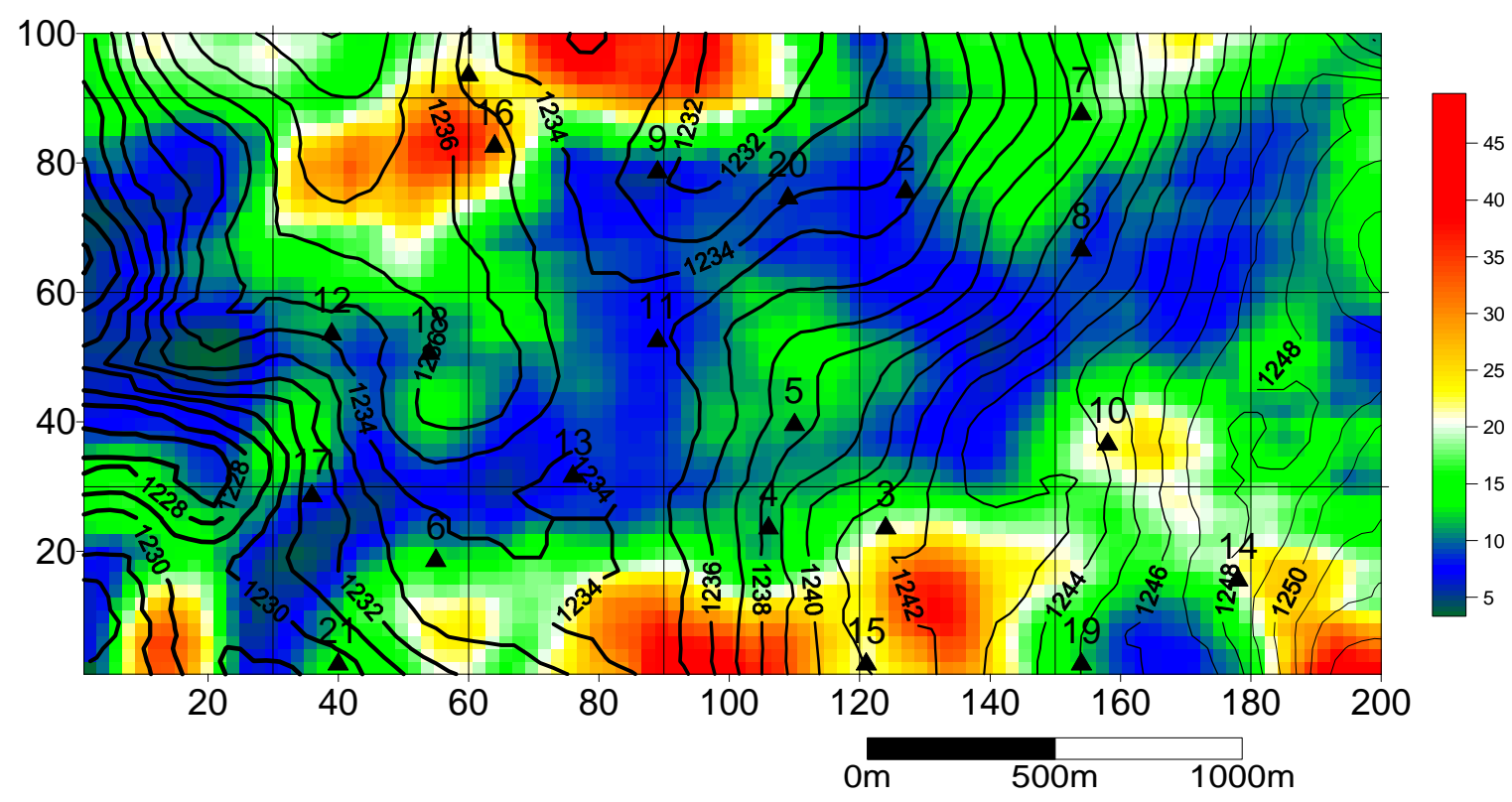

Figure 27: DFM results for Stratton field

An apparent channelwas identified (TSI) by its stress concentration (high effective stresses in blue) along an interval at about 1250ms, labeled SH2 on Figure 9; Contours are time. 


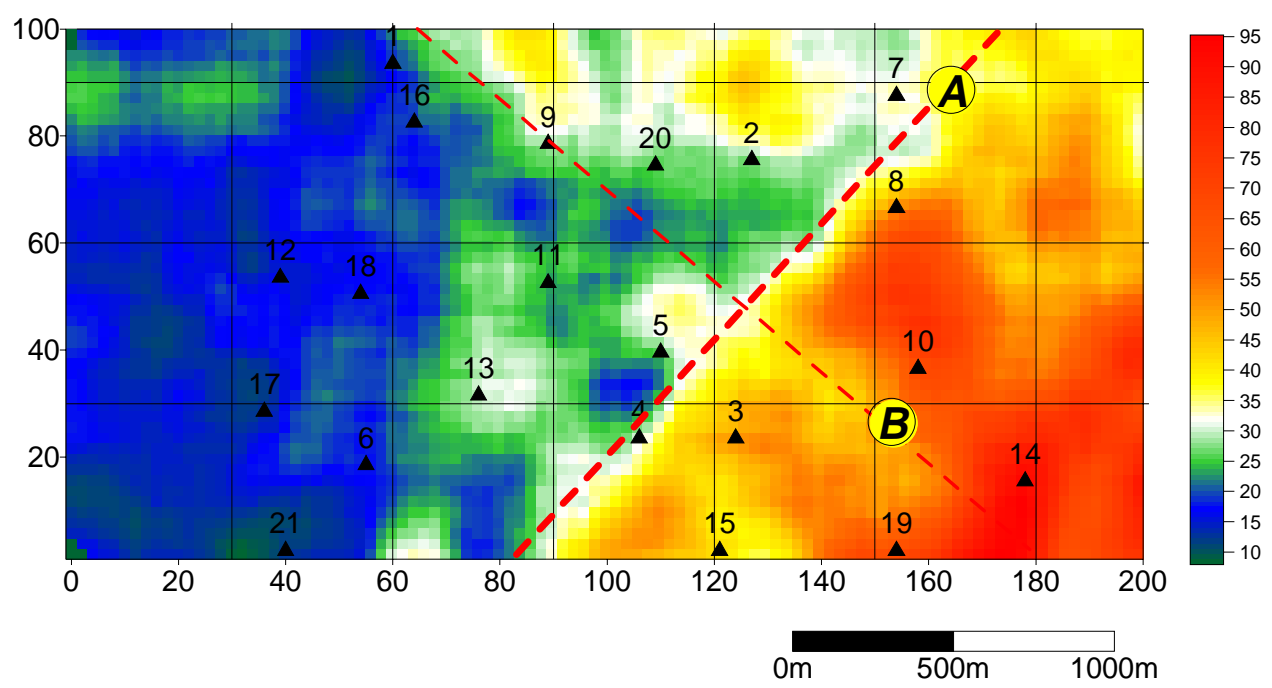

Figure 28: DFM results for Stratton field showing the block structur.

Inferred in the deeper Vicksburg formation. TransSeismic International has concluded that these deep-seated structures control the development of channels throughout later times, and their boundaries provide pathways for fluids.

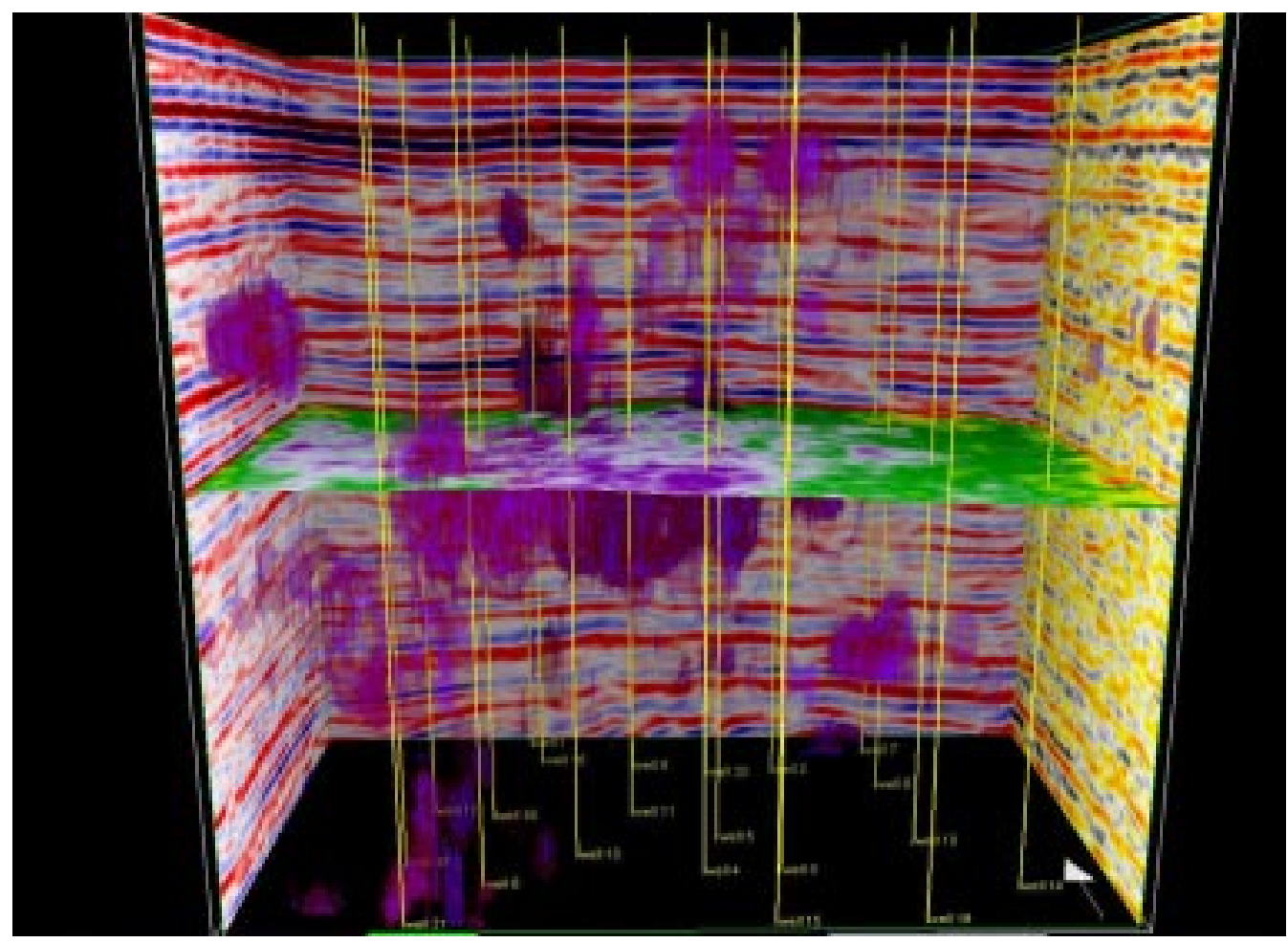

Figure 29: DFM results for Stratton field showing regions of low effective pressure as purple $3 D$ bodies. 


\section{Boonsville Field, North Texas}

This study area is the Boonsville Field of the Fort Worth Basin in north central Texas (Figure 16a). The data set includes 5.5 square miles of 3-D seismic data set and 38 wells with modern logs , made available through the Texas Bureau of Economic Geology (Hardage et al., 1996a). Here we concerned ourselves within the Caddo sequence of the Pennsylvanian Group (Figure 30) although other productive sequences also exit in the area.

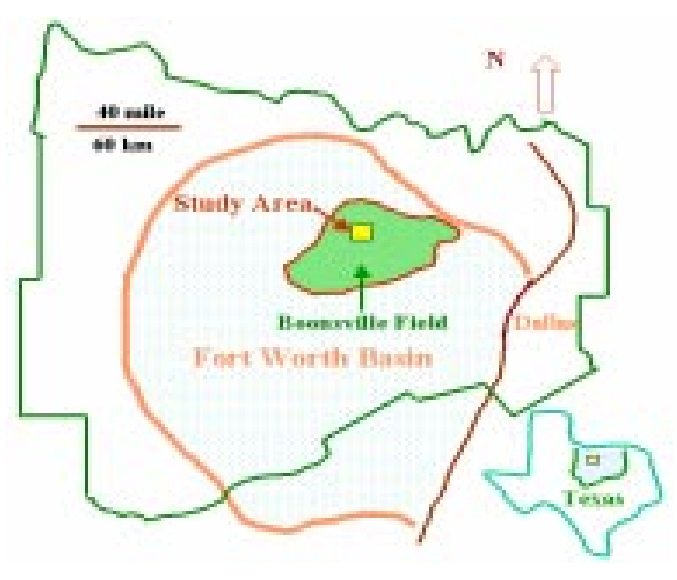

Figure 30a: Location map of the Boonsville study area.

Figure 30b. Traverse seismic line through the main delta showing the Caddo sequence.

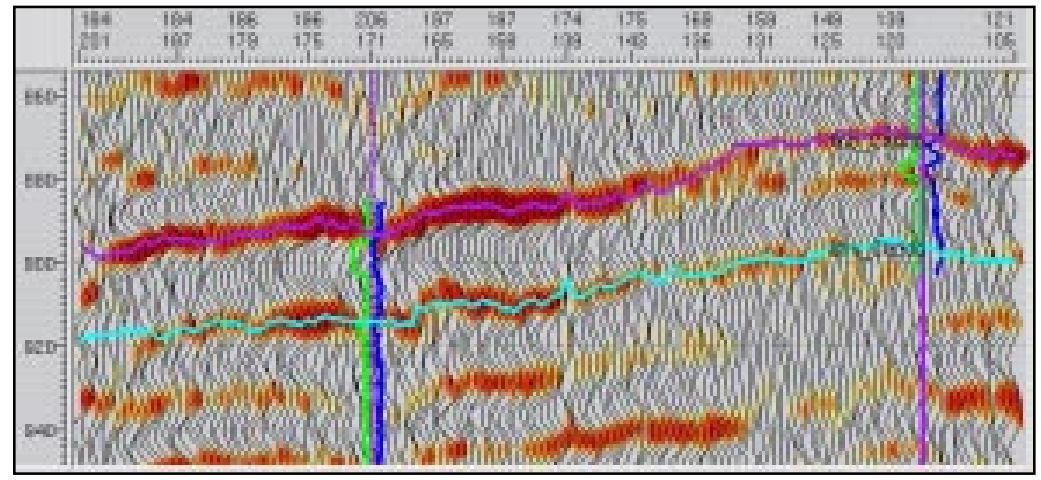

The reservoir characteristics and the related data set meet our basic requirements to ensure reaching the research goals. Some features of the targeted Caddo sequence are: hydrocarbons are produced from the thin-bed sandstone reservoirs with thicknesses totaling 0 to 52 net feet of the Caddo sequence, with a gross thickness of 80-150 feet; the tight limestones have known thicknesses of 0 to 30 feet and appear similar to the thin-bed sandstones at seismic scale; the depositional environment is dominated by a delta system; both the sandstone and limestone are embedded in the shale-rich sequence and characterized by higher P-wave velocities and higher densities, thus generating higher acoustic impedances; both the sandstones and limestones are highly variable laterally and vertically.

Our primary research goal is to establish a depositional model of the thin Caddo sequence using the 3-D seismic data integrated with well data. In this study, we first build a depositional model using the well data only, and then examine some existing approaches to the use of seismic data for facies analysis, such as trace coherence and classification. A new method will be presented to recognize the subtle geological and geophysical features of the Caddo sequence, based on crosscorrelation of seismic traces with one or more traces believed to represent specific depositional environments.

The original work by Hardage et al (1996a) emphasized the importance of karstic features in formations below the Caddo sequence. Some of these features are shown in Figures 31 and 32. Our study was concentrated on depostional features and their recognition in the Caddo sequence above the karsted formations. 


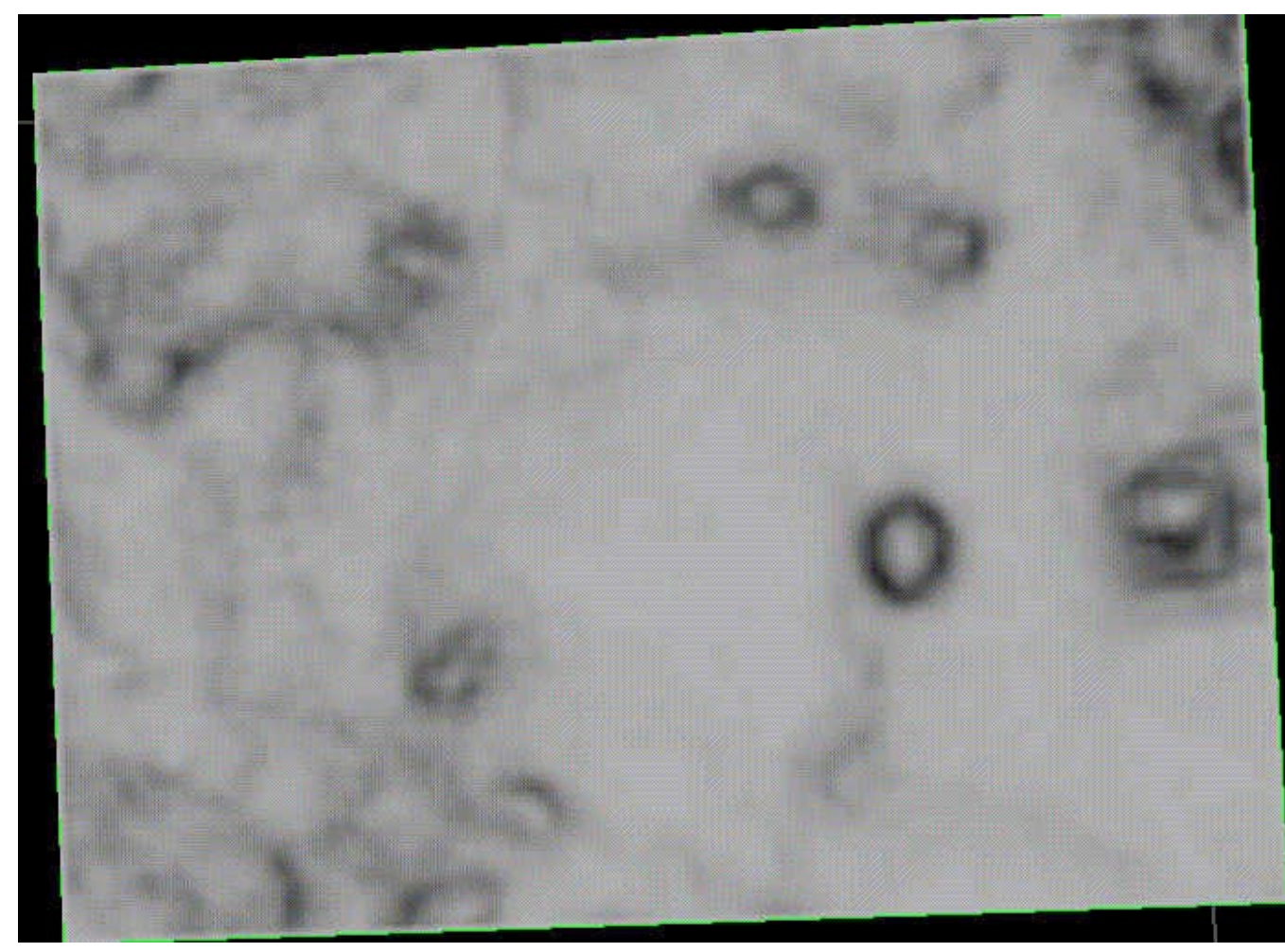

Figure 31:

Coherence

(variance).

Image of a

time-slice

through the

Boonsville

data set

showing the

presence of

karstic

features, including sinkholes, underlying the Caddo horizon.

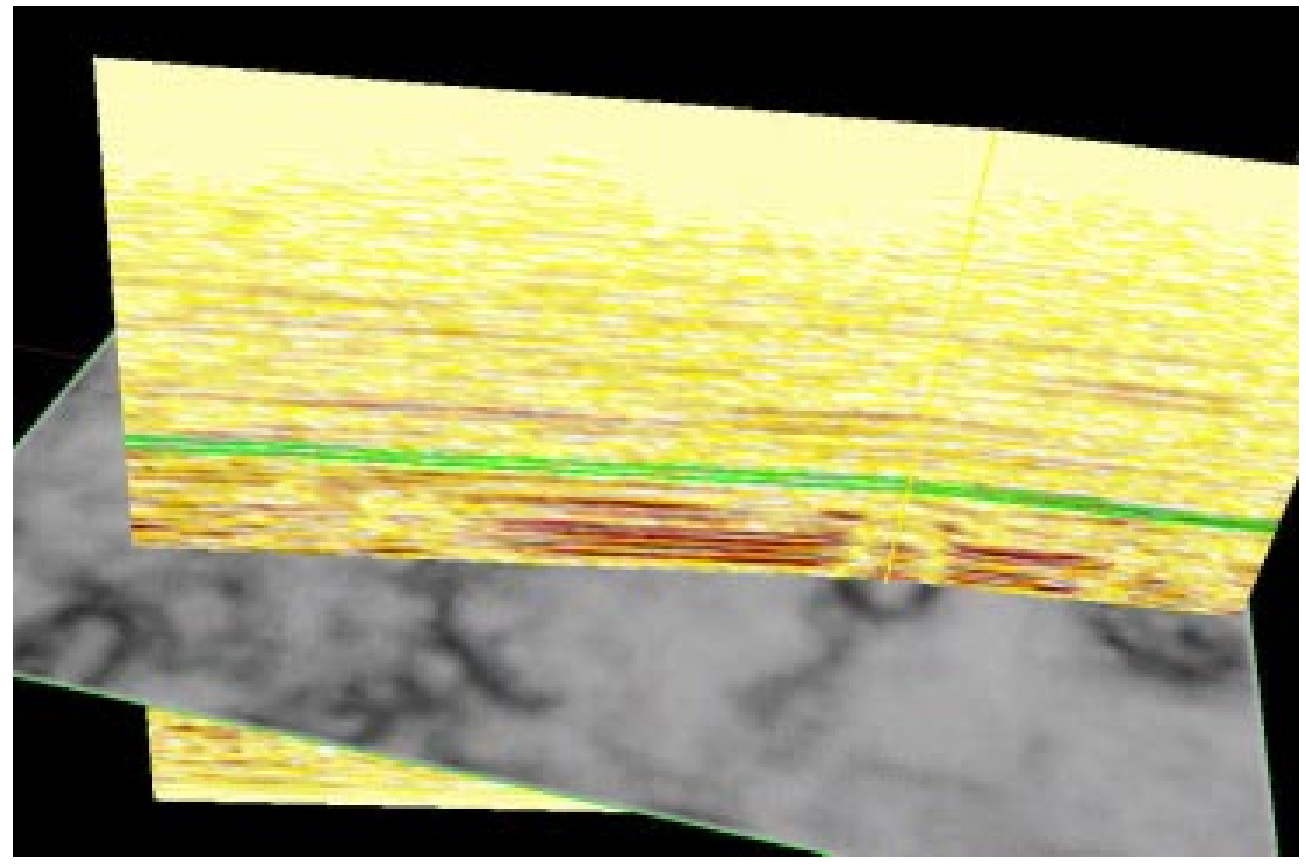

Figure 32: 3D visualization of the Boonsville data set

Showing the relationship of the karstic features to other reflections and the Caddo sequence (green horizon). 


\section{Boonsville Field 1: Quantitative seismic facies analysis for thin-bed reservoirs}

In this section, we describe a new pattern recognition model developed to recognize the subtle geological and geophysical features of a thin-bed sequence and predict thin-bed reservoirs, based on cross-correlation of seismic traces with one or more traces believed to represent specific depositional environments. Our approach has the following characteristics: (1) It corrects for the possible mistracking of top horizons by searching about a time window specified by the user; (2) It presents seismic facies as continuous values by introducing the modified cross-correlation algorithm, as opposed to a limited number of clusters; (3) It more easily performs one or more supervised trace pattern recognition and combines each of them into a seismic facies.

\section{INTRODUCTION}

Using 3-D seismic data integrated with well data to build reservoir depositional model is routine work and seismic stratigraphy plays a significant role, albeit in a qualitative fashion. However, it is not uncommon for thin sequences to exhibit seismic trace pattern changes that are subtle and with ill-defined termination patterns. Our case study area poses an additional challenge: how to distinguish thin-bed reservoirs and thin-bed non-reservoirs when they appear similar at seismic scale. It is this second challenge that we address in this section.

The average acoustic impedances of the shale, sandstone, and limestone of the Boonsville Field are 30000,36000 and $40000 \mathrm{ft} / \mathrm{s}^{*} \mathrm{~g} / \mathrm{cc}$ respectively (Figure 33), indicating that we could separate the sandstone reservoirs from the limestone non-reservoirs if we were capable of identifying their acoustic impedance with any confidence. However, the discrimination is very difficult in this field because the limestone and sandstone layers appear similar at the seismic scale.
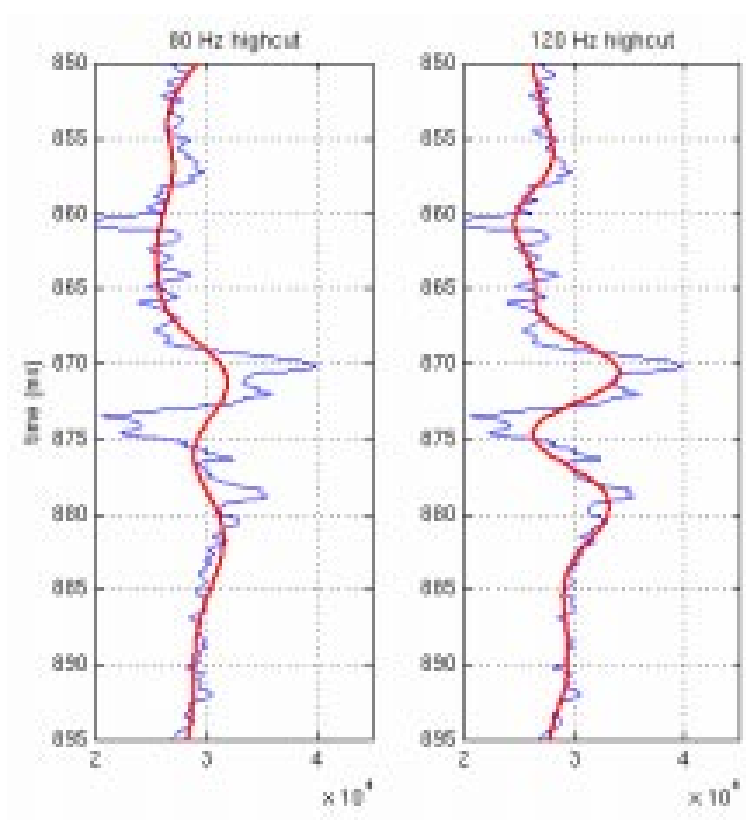

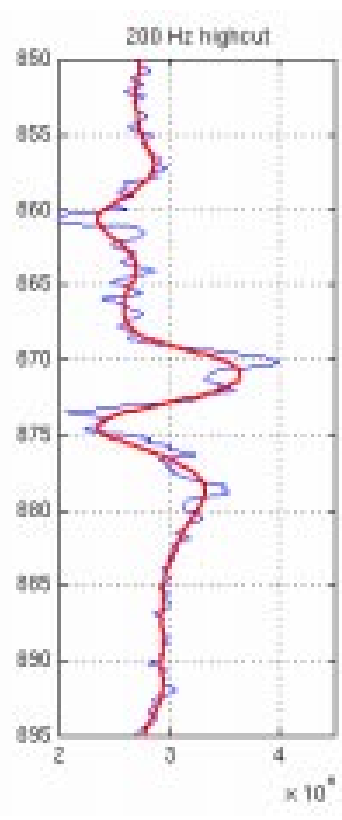

Figure 33: Sample impedance contrasts in Boonsville field.

The fast bed at about $870 \mathrm{~ms}$ two-way time is limestone, and the fast bed at $878 \mathrm{~ms}$ is a sandstone; the other formations are mostly shales. The original impedance is shown in blue on all traces, and the filtered impedance is shown in red, using a highcut filter as labeled. Notice that below $120 \mathrm{~Hz}$, the distinction between the limestone and sandstone beds is insignificant.

A sonic well log has a resolution less than 5 feet in comparison with seismic resolution, up to 50 feet for the Boonsville data set. The frequency bandwidth of the Boonsville 3-D seismic data is from 15 to $120 \mathrm{~Hz}$ while the frequency bandwidth of the velocity log ranges from 0 to greater than $500 \mathrm{~Hz}$. High-cut filtering is required to "upscale" the frequency bandwidth of the well data 
to the seismic data. As a result of high-cut filtering, the high impedances of the thin-beds are averaged with nearby lower impedances. The final effect is that the higher impedance of the limestone non-reservoir appears to be close to the impedance of the sandstone reservoir. A more complete treatment of the problem would include Backus (1962) averaging of the thin beds, with details depending on the frequency content of the wavelet, but the net effect will still be the overall reduction of the highest impedance resolved. [This approach is considered later in this report, with the Wamsutter data set.] Therefore, two rock types may easily appear similar at seismic scale and become difficult to distinguish through the interpretation of a seismic data set alone, even though the actual thin-bed impedances are significantly different

\section{Well-Based Depositional Model}

The depositional system of the Pennsylvanian Atoka Group has been studied by Thompson (1982; 1988), Glover, (1982), and Lahti et al. (1982). However, these studies were mainly focused on the entire Fort Worth Basin. Hardage et al. (1996b; 1996c) and Carr et al. (1997) analyzed the reservoir distribution and depositional subfacies using a few typical wells from this study area, but their model is not sufficiently detailed to be useful for facies analysis of this thin Caddo sequence. In addition, the tight non-reservoirs were generally not studied even though both the limestones and reservoir sandstones exist and appear seismically similar in the Boonsville Field. Therefore, a depositional model was established with a concentration on reservoir distribution and the discrimination of the sandstone from the limestone. The techniques involved include well log trend recognition and lithology determination.

The well log trends and lithologies analysis are summarized in Figure 34 and the well log trends from representative wells are illustrated in Figure 35. The main delta system was recognized in the east and the possible second delta system was observed at the northwestern corner, but appears to be mostly outside of the study area (Figure 35).

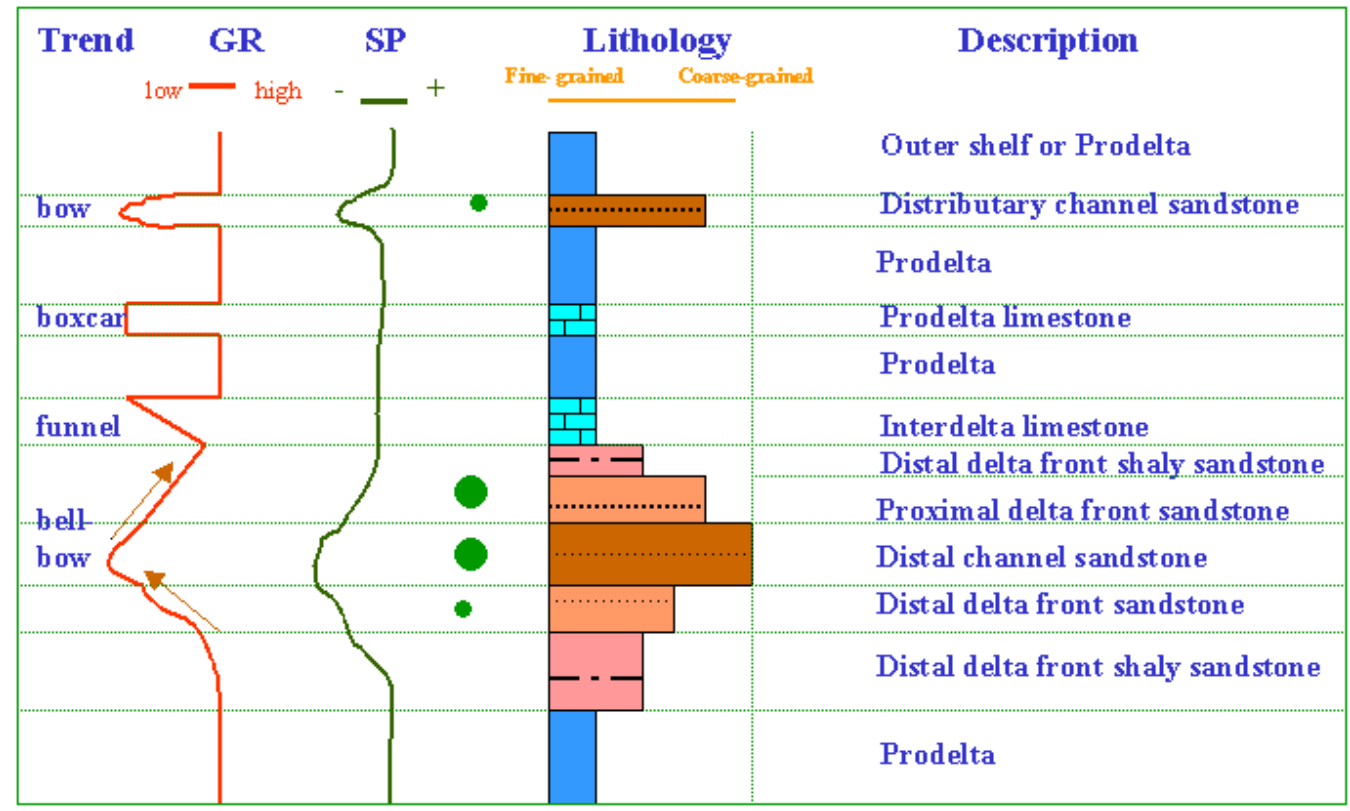

Figure 34: Generalized well log trends and depositional subfacies and lithologies of the Caddo sequence. 


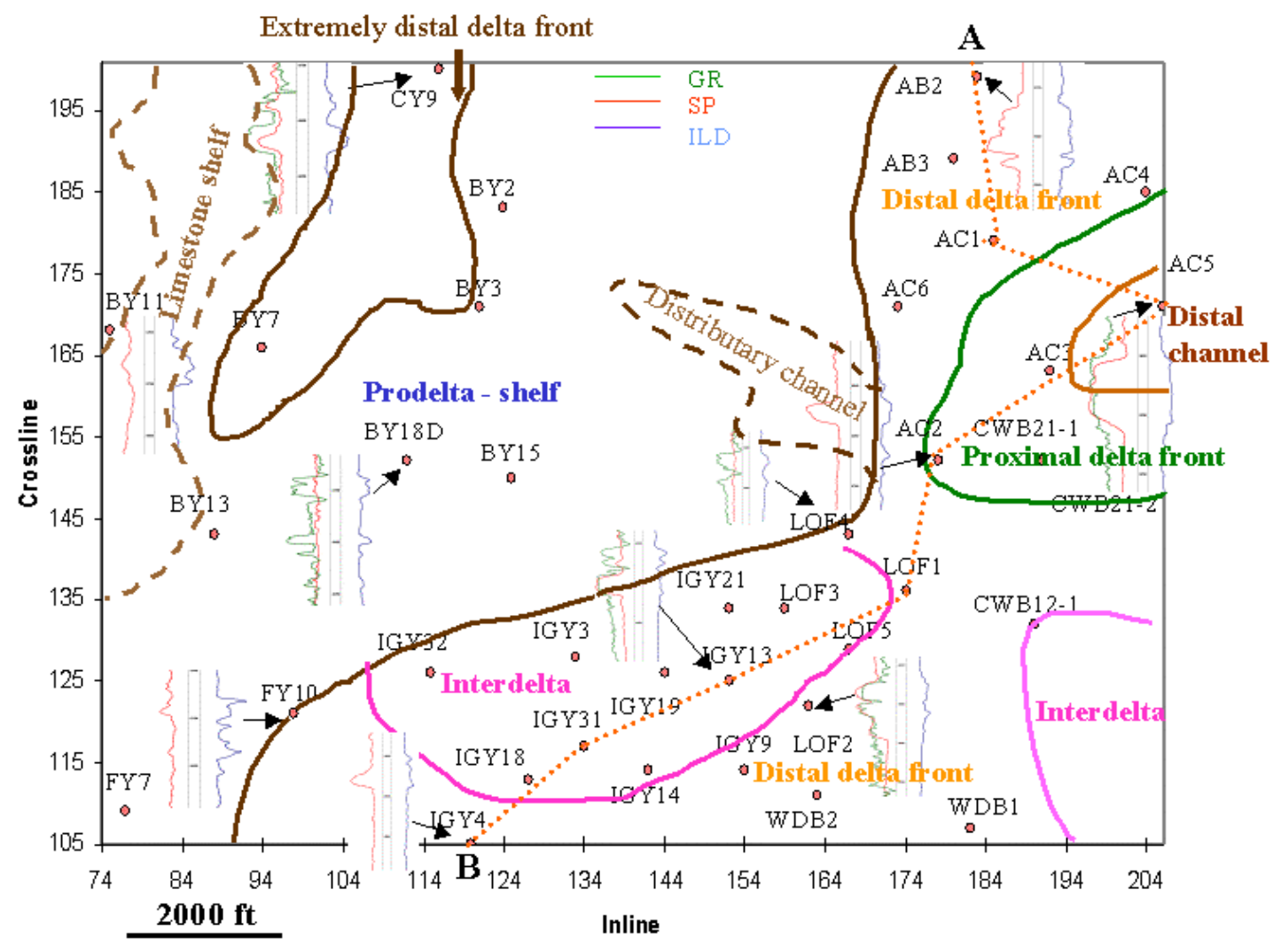

Figure 35: Representative well log trends and depositional subfacies of the Caddo sequence based on well data, with a possible limestone shelf and distributary channel based on seismic inversion. Cross-section A-B is indicated by the dashed line, and used in other figures. (Modified from Coleman et al., 1982; Thompson, 1982; Emery et al., 1996; Hardage et al., 1996b,; and

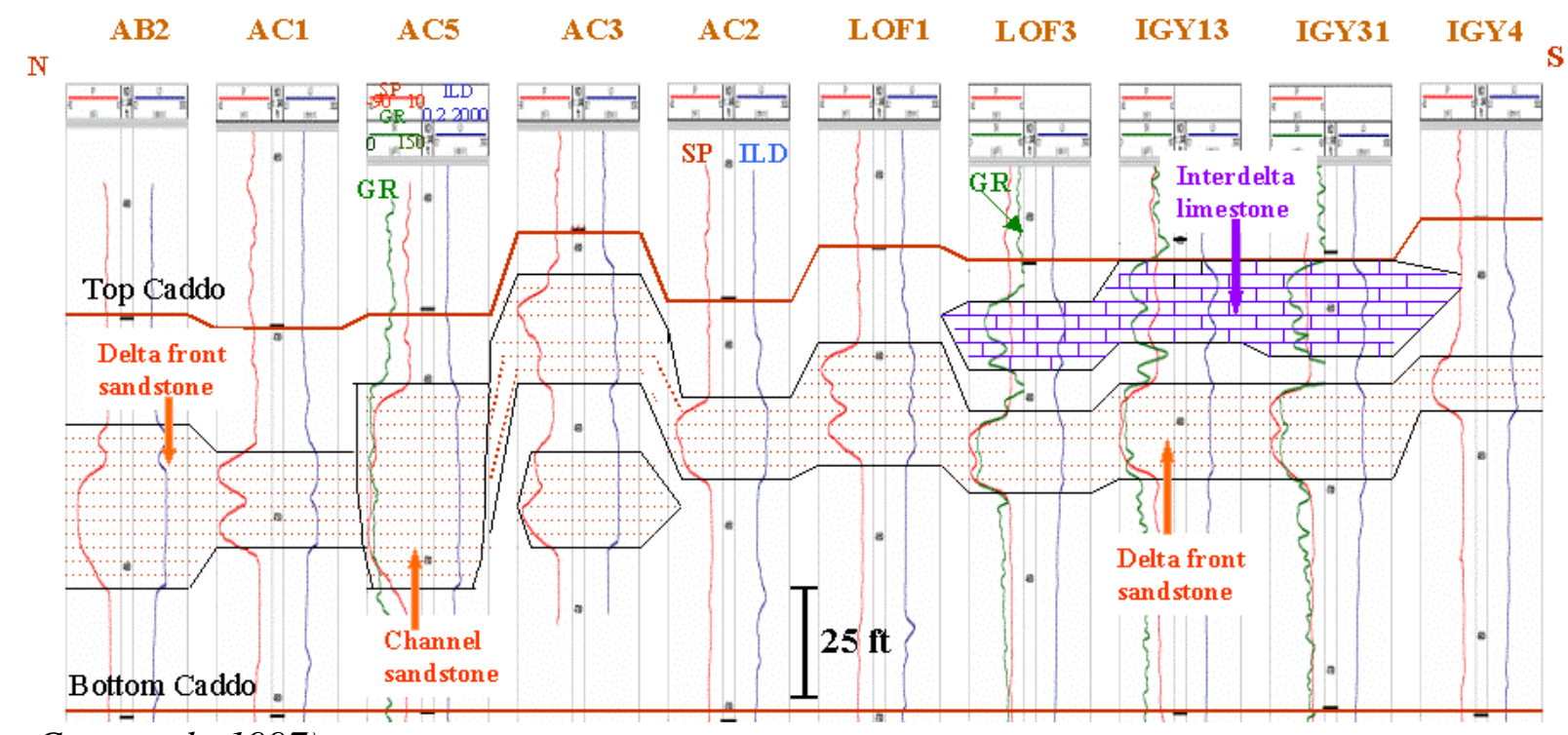

Carr et al., 1997)

Figure 36: Well correlation and interpretation; flattened on the Bottom Caddo marker. 
The main delta system was divided into three delta subfacies: distal channel in the east margin, proximal delta front in the east, distal delta extending to south and northeast, and interdelta in the south and southeast. As depicted in a cross-section through the main delta system (Figure 36), the depositional subfacies and reservoir distribution in the eastern oil producing region are characterized by the distal channel and the proximal delta front are the depositional subfacies in which the thickest sandstone reservoir (up to $52 \mathrm{ft}$ ) was deposited; the distribution of limestones is limited, but the limestones can be correlated as the GR log trend is similar and correlative; the sandstone reservoirs are widely distributed in the delta front subfacies.

The second delta system observed from the well data was detected at the northwestern corner. However, the main delta body remains outside the study area (Figure 35) and the relationship of this delta system (or sub-system) with the main delta (sub)system in the east remains unclear.

The rest of the region is dominated by the prodelta or shelf, filled with shales and limestones. The SP log trend is a flat base line while the GR log pattern is a "boxcar". The "boxcar" indicates the prodelta limestone. No good reservoir sandstone was found in the prodelta subfacies, and instead, the shaly sandstones were recognized and no oil was produced from them. The maximum known thickness of the limestone in prodelta subfacies is 30 feet at well BY11 and BY13. However, the simulated acoustic impedance data reveals that a narrow north-southoriented thicker limestone zone might exist in the west that will be discussed later.

\section{SeISMiC tRaCe PATTERn AND Depositional Model}

Chen et al. (1997) suggested that more than 200 seismic attributes (not all of them are independent) would be useful in constructing reservoir depositional model. Other researchers (Horkowitz, et al., 1996; Hardage et al., 1996b; Cooke, et al., 1999) claimed that instantaneous frequency and/or instantaneous phase are effective at highlighting facies changes. Similarity analysis based on multiple point-based attributes, their means and deviations was used to map seismic facies (Michelena, et al., 1998). However, these point-based attributes only describe one aspect of a complicated seismic trace shape. The trace-based attributes may be a natural solution for better defining facies. In this study, two widely used approaches, coherence and trace pattern classification will be examined before a new technique is presented and tested.

Like the well-log trend, seismic trace pattern can be used to define a depositional facies. Notice that the patterns of the synthetic traces created from velocity and bulk density well logs do not perfectly match the real seismic traces because of different measurement scale, assumptions, and noise involved. However, they indicate that different depositional subfacies and reservoir distribution correspond to the changes in seismic trace patterns, which is the petrophysical basis for using seismic trace pattern to construct depositional subfacies.

\section{SEISMIC TRACE COHERENCE}

Seismic trace coherence is a measure of lateral changes in the seismic trace pattern and is based on a cross-correlation measurement. Bahorich et al. (1995) invented this technique and has since been modified by introducing semblance, structural dip and azimuth (Marfurt et al. 1998; 1999). Bahorich et al. (1995), Marfurt et al. (1998; 1999), Cooke (1999), and Chopra (2001) demonstrated that coherence can be used to define stratigraphic features. Our study suggested that the resolution depends upon the scale and the degree of variations in the patterns of neighboring traces. In particular, coherence may not be helpful if the changes in the patterns of 
neighboring traces are gradual, in which case, all of the coherence values are almost identical and no features are apparent. Furthermore, because the algorithm compares the neighboring traces along inline and crossline directions or traces in small regular-shaped (elliptical or rectangular) analysis regions (tessellations) at each iteration, it cannot use specific traces for correlation through the volume.

A coherence cube from the Boonsville data set was obtained (Figure 37; also Figures 31 and 32). The delta depositional feature has not been recognized because its variations are subtle; on the other hand, the effects of some deep karstic features are apparent in the coherence volume, even at the Caddo level. As expected, the stratigraphic or structural features stand out if the changes are sharp. Whenever this is the case we can interpret them in a conventional reflection data volume, provided that a coherence cube makes these features more visible for interpretation. Overall, coherence seems to be most useful in configuring depositional systems which involve significant changes in seismic trace pattern, and in interpreting structures because structural deformation often sharply distorts seismic trace patterns.

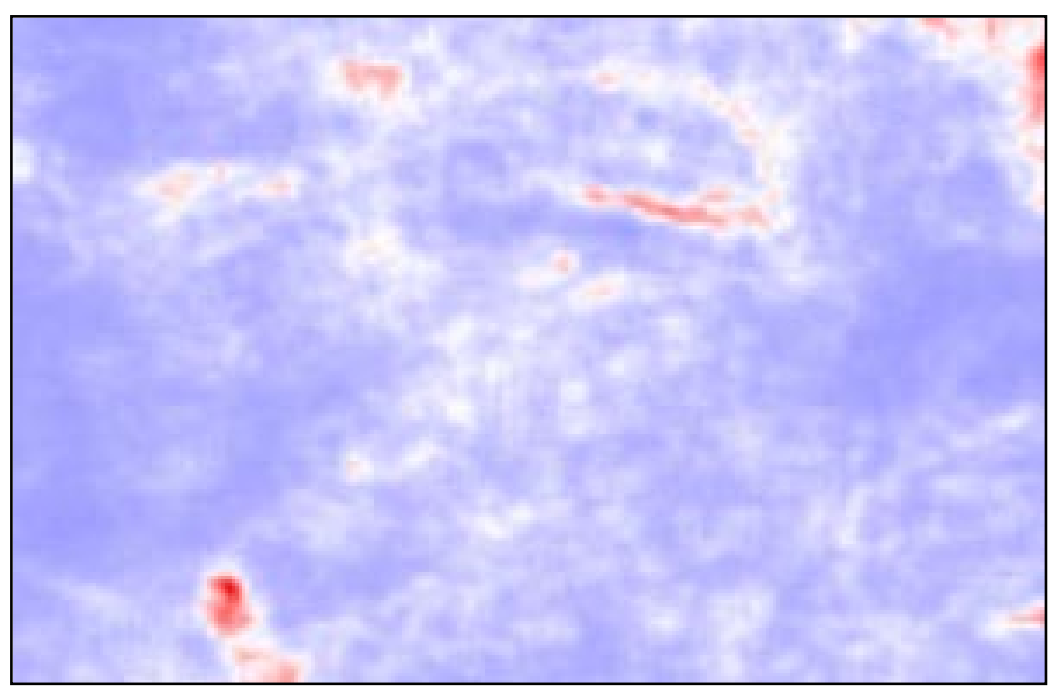

Figure 37: Seismic coherence time slice at $890 \mathrm{~ms}$ within the Caddo sequence.

\section{SEISMIC TRACE PATTERN CLASSIFICATION}

This approach classifies all seismic traces in a survey to a limited number of classes using mathematical algorithms which vary from conventional cluster analysis to neural network classification. One popular algorithm is based on Kohonen self-organization map (SOM) neural network recognition (Kohonen, 1990; Gurney, 1992). Poupon et al. (1999) demonstrated its usefulness in seismic facies analysis. Basically, in this scheme, each of the model classes corresponds to a discrete class of patterns and the problem then becomes a decision process. All model classes are "self-organized" and updated at each iteration. The final classes are assigned to each trace, each of them is labeled with the corresponding model classes or colors (the lower panel in Figure 38). Notice that changing the time of first sample in the series, due to horizon mistracking, can significantly alter the vector value.

The number of model classes for the neural network needs to be optimized by maximum difference among each model class and maximum correlation factor. Five, ten, fifteen, and twenty classifications were initially chosen with $30 \mathrm{~ms}$ tine window, and the ten-classification result provided a map that correlated best as the correlation factor is greater than $70 \%$ in most of the study area. The patterns can be correlated to the depositional subfacies (upper panel in Figure 
21) if properly calibrated from the well data (Figure 35). Patterns $4-10$ were correlated to the delta front and patterns 9-10 were compared to the inter-delta subfacies, patterns 4-8 to the prodelta subfacies. Patterns 5-6 in the west represent thicker limestone zone in the prodelta subfacies. Again, notice that each of the pattern groups is meaningful with depositional subfacies because they were properly correlated to the well-based depositional subfacies.

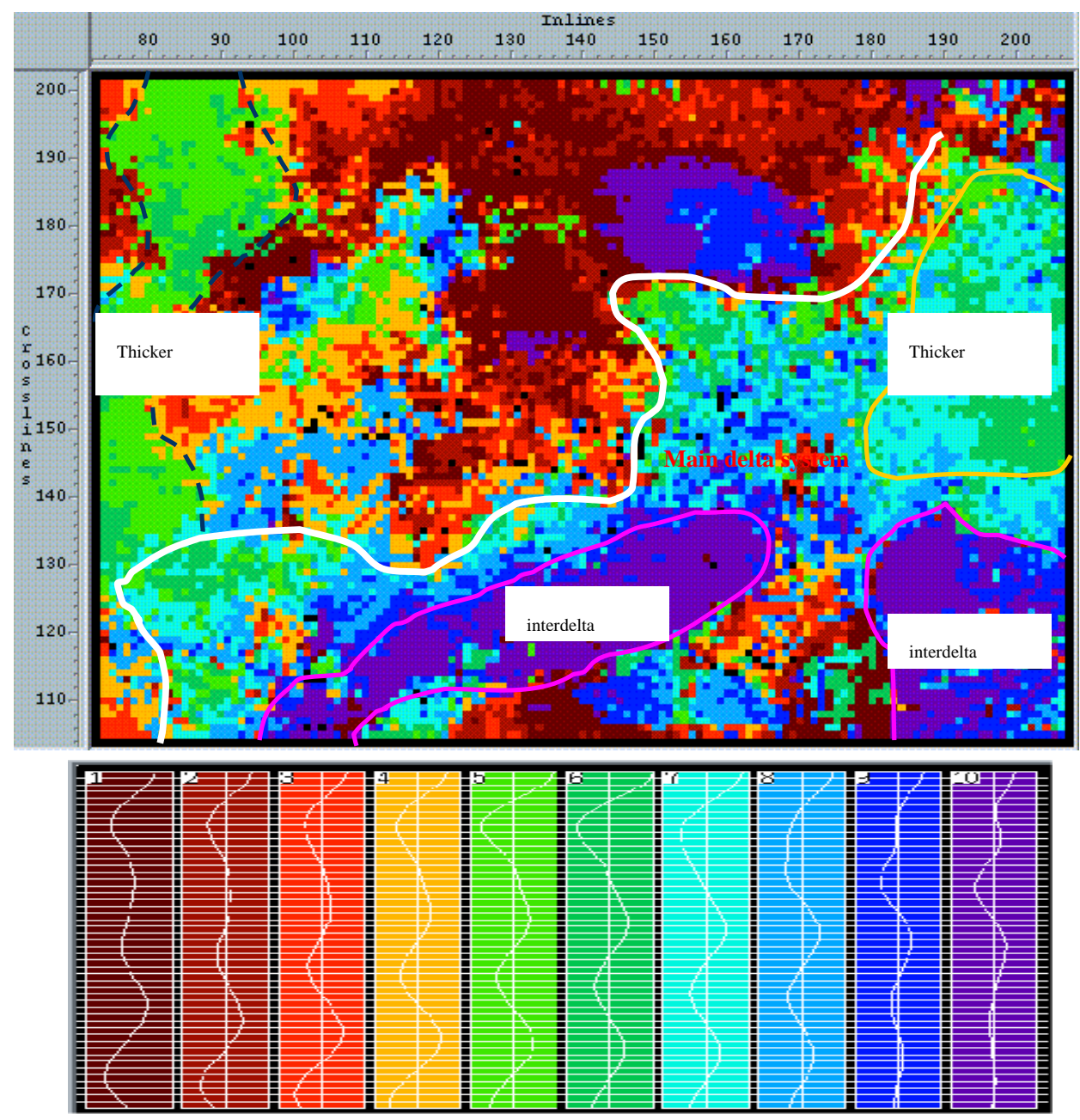

Figure 38: Seismic trace pattern classification and correlated depostional subfacies of the Caddo sequence (upper panel) and ten trace model classes for the seismic trace classification (lower panel, $30 \mathrm{~ms}$ window from the top Caddo).

\section{A New Agorithm for Seismic Facies Analysis}

We developed a new approach which can: (1) realign a mistracked horizon, (2) discern subtle changes in seismic trace patterns, (3) easily perform pattern recognition for user-specified traces over a survey, (4) provide continuous output values, and (5) combine and visualize the results for multiple trace pattern analysis (posterior-classification).

The algorithm is a modified cross-correlation model, which is a standard method for estimating the degree to which two series are correlated. Consider two series of signals X(i) and Y(i) where $\mathrm{i}=1,2, \ldots \mathrm{N}$. The cross-correlation, $\mathrm{R}$, at delay $\mathrm{d}$ is defined as 


$$
R=\frac{\sum_{i}^{N}\left[\left(X(i)-X_{m}\right)\left(Y(i-d)-Y_{m}\right)\right]}{\sqrt{\sum_{i}^{N}\left(X(i)-X_{m}\right)^{2}} \sqrt{\sum_{i}^{N}\left(Y(i-d)-Y_{m}\right)^{2}}}
$$

where $X_{m}$ and $Y_{m}$ are the means of the corresponding series and $d$ is the time window for possible horizon mistracking. The denominator in the expressions above serves to normalize the correlation coefficients such that it ranges from -1 to 1 . A value of one indicates maximum correlation while zero indicates no correlation. A high negative correlation exhibits a high correlation but of the inverse of one of the series. However, this cross-correlation is focused on the relative similarity of patterns between two time series rather than absolute similarity. Hence, this expression was modified such that it can judge the difference in absolute values within the shape. The modified expression is written below, showing an additional factor that computes for similarity of amplitude on an absolute value:

$$
R=\left(\frac{\sum_{i}^{N}\left[\left(X(i)-X_{m}\right)\left(Y(i-d)-Y_{m}\right)\right]}{\sqrt{\sum_{i}^{N}\left(X(i)-X_{m}\right)^{2}} \sqrt{\sum_{i}^{N}\left(Y(i-d)-Y_{m}\right)^{2}}}\right)\left(\frac{\sum_{i}^{N}|Y(i)|}{\sum_{i}^{N}|X(i)|}\right)
$$

This algorithm, as implemented, can also correct for possible horizon mistracking by searching an amount of time (d samples specified by the user) in order to find the highest value for R. The output values for $\mathrm{R}$ are continuous from -1 to 1 and provide a value at every trace.

The input of this model consists of seismic data and horizon file as provided by the user. The user needs to define the possible mistracking time window which depends on the confidence of the horizon tracking. The users must then input a trace location of interest, usually a trace at a well with known lithology and/or depositional subfacies. The program will perform pattern processing and output the results, indicating the similarity of all other traces to the one specified trace. Results for one trace at a time are shown in Figure 39.

In general, one-trace pattern recognition provides a good seismic facies map as shown in Figure 37 derived from well AC3 only, with R values ranging from -0.8 to 1 . Three seismic facies types are readily identified, and by consulting the well-based depositional model (Figure 18), the association with geological facies can be determined with ease. The area of the map with R-value correlation above 0.7 indicates that all the trace patterns are close to the trace pattern at well AC3, which is indicative of the main delta system (notice that the trace patterns were distorted at the northeast corner where karst and faults were developed). Two isolated areas in which the correlation is low or negative indicate the inter-delta subfacies. The coefficient in the north is also low, ranging from -0.3 to 0.7 , and is correlated to the prodelta subfacies. Notice that the time window used for the pattern processing is $30 \mathrm{~ms}$ starting from the top Caddo sequence, the same size as the previous trace classification window.

Various traces at different well locations were chosen to provide trace pattern processing for different depositional facies. Well CWB21-1, also indicative of the main delta system was used, and the results are almost identical to the facies map derived from well AC3. Well IGY31 recorded the inter-delta subfacies and its corresponding seismic trace pattern correlation results are consistent with the well-based map. Notice that other high coefficient values in the central 
north area were detected where structural deformation (i.e., karst) was also identified with a strong correlation to this well, a result that is perhaps coincidental.
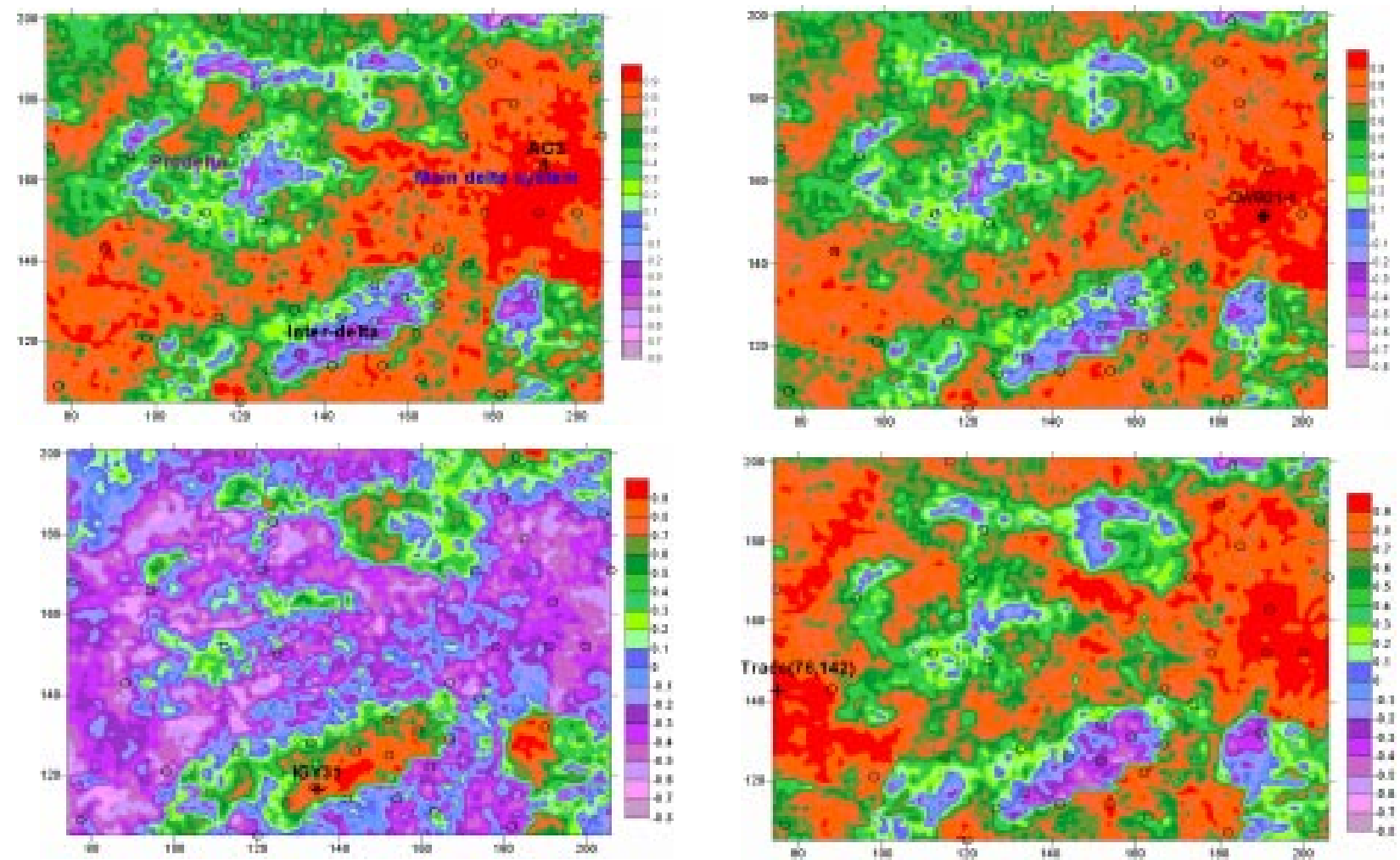

Figure 39: Seismic facies based on new algorithm, using one trace at a time for classification.

Upper left: based on well AC3. Lower left: based on well IGY31
Upper right: based on well CWB21-1. Lower left: based on trace Inline 76, Crossline 142

This algorithm enables us to process any trace specified by the user, whether or not it is at a well location. The trace at inline 76 and cross line 142 is believed to be located within the thicker limestone zone. The high coefficient values indicate the thicker limestone distribution in the west. Notice that the trace patterns in the east where the thicker sandstone reservoirs were developed also correlate with the limestone-based trace. Our algorithm can now be extended to help remove this apparent ambiguity.

Although this new approach is capable of producing reliable seismic facies maps using only one seismic trace for pattern processing, the results from multiple user-specified trace pattern processing can be combined and visualized. The following expression is used to meet this need.

$$
\mathrm{R}=\mathrm{i} * \max \left(\mathrm{R}_{1}, \mathrm{R}_{2}, \ldots \mathrm{R}_{\mathrm{i}}, \ldots, \mathrm{R}_{\mathrm{n}}\right)
$$

where $\mathrm{i}$ is the order of seismic trace chosen to perform the processing and $\mathrm{n}$ is the number of the traces selected. $R_{i}$ is the correlation coefficient based on the selected trace with the order of $i$, that is, $R_{1}$ may be the correlation coefficient for a trace exemplifying the main delta, whereas $R_{2}$ represents the inter-delta, $\mathrm{R}_{3}$ the limestone, and so no. The resulting $\mathrm{R}$ is then chosen as that value, the largest from among the correlations made; it is also assigned a value $(i)$ identifying which facies it represents. 
Figure 40 depicts the combined seismic facies obtained from three pattern-processed seismic traces, that is, the traces at well AC3, IGY31 and BY11. These three wells represent the main delta, inter-delta, and prodelta depositional subfacies, respectively. The figure also demonstrates the ability to discriminate the thicker sandstone reservoirs in the east and thicker limestones in the west by adding the additional facies from traces in the known limestone region and thick sandstone region.

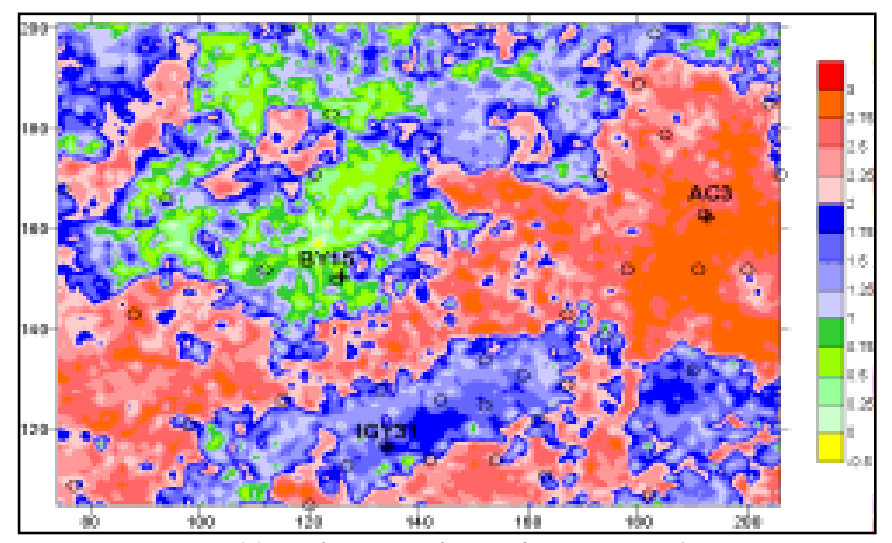

Figure 40: The combined seismic facies using multiple traces.

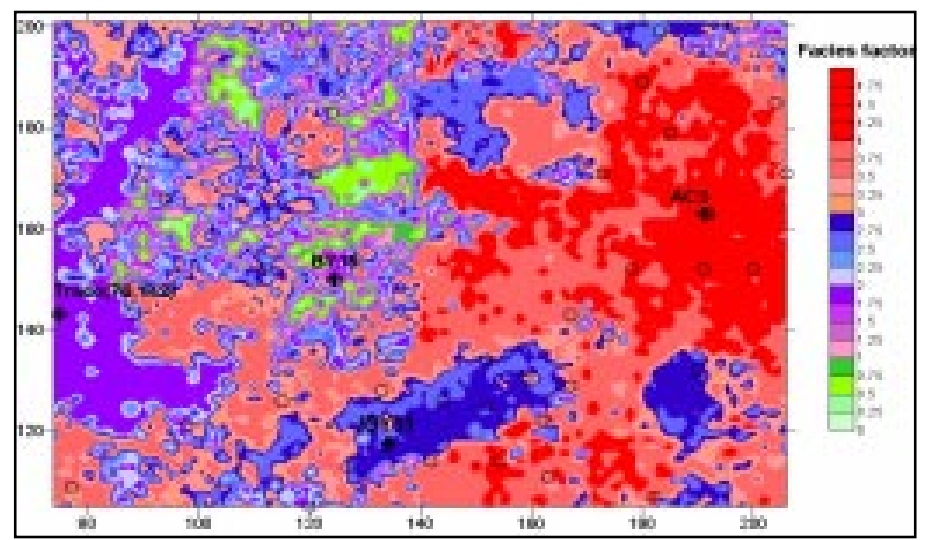

Right: limestone and sandstone traces added.

\section{CONCLUSIONS}

The seismic trace coherence may not able to reveal subtle depositional facies in a thin sequence because of gradual changes of the patterns among the closest neighboring traces. The trace classification approach based on the neural network clustering provided very good results. Our new approach appears to provide some advantages. It provides pattern recognition for multiple user-specified traces (for example, specific "depositional trace" or "oil trace" and "gas trace") over an entire survey. The algorithm has been demonstrated, via the case study, to be robust and promising in defining seismic facies of subtle geological features and predicting thin-bed reservoirs. Notice that, similar to every geophysical output, the geological meaning needs to be defined or verified by geological information. The same correlation values may not represent the same depositional subfacies and the assignment of depositional subfacies depends on our confidence on the geology. More wells, if available, should be selected for the multiple trace pattern processing in those less confident regions.

\section{Boonsville Field 2: Seismic Attributes for Thin-bed Reservoir Prediction}

The conventional thin-bed tuning model (i.e., the wedge model) is examined with emphasis on its usefulness or possible pitfalls in thin-bed reservoir prediction of the Caddo sequence, and a new non-linear statistical inversion model built on the generalized regression neural network (GRNN) is applied to the Caddo sequence. Two other seismic inversion models, a non-linear statistical model based on the probabilistic neural network (PNN) and a deterministic model using constrained sparse spike inversion (CSSI) are used to delineate the reservoirs of the Caddo sequence. Our study shows: (1) The conventional thin-bed tuning model is appropriate only if one single thin-bed is developed or multiple widely-spaced thin beds (greater than one quarter of the wavelet length) are present in the sequence of interest. In other words, the model should not be applied for multiple closely-spaced thin beds because of significant destructive interference; 
(2) All three seismic inversion models were able to identify the thicker reservoir sandstones. However, the resulting details for the thin beds vary. The sparse spike inversion with constraints provided few indications of the thin-bed distribution although it was largely noise-free. The probabilistic neural network (PNN) delineated most of the thin-beds, but produced more noise as well. Our GRNN method predicted the thin beds at 13 out of 20 wells. The GRNN inversion method can be very useful in detecting thin-bed reservoirs in existing fields where a number of wells are available.

\section{INTRODUCTION}

Reservoir characterization has historically focused on thick reservoirs (i.e., the thickness greater than seismic resolution). One of the most challenging research problems is to characterize thinbed reservoirs using 3-D seismic and well data. Based on the quarter wavelength criterion, thinbed reservoirs range in thickness from a few feet to tens of feet. Tuning effects are observed at this thickness range due to wavelet interference (Widess, 1973; Brown, 1996) and thin-bed reservoirs may not be detected in seismic data or may be difficult to integrate with well data. However, thin-bed reservoir characterization is important as the petroleum industry is devoting greater effort toward tapping more oil from existing fields, many of which are in thin-bed environments.

Progress is being made towards characterization of thin-bed sandstone reservoirs using: (1) instantaneous seismic attributes (Hardage et al. 1994, 1996a; Horkowitz et al., 1996); (2) amplitude (Cooke et al. 1999; Hoover, 1999); (3) frequency decomposition (Partyka et al., 1999); and (4) Bayesian simulation (Gastaldi et al., 1998). To characterize thin-bed reservoirs, however, there are two key factors need to be taken into consideration. One is that a single reservoir layer or multiple widely-spaced reservoir layers are rarely developed in a sequence of interest. Closely spaced thin-bed layers are difficult to predict using the conventional thin-bed tuning model which was generated for a single thin bed between two thick beds. The second one is to remove the tuning effects (detuning). Reflections result from the convolution of a source wavelet with a reflection coefficient, thus representing the boundary between two layers instead of the layer properties. Seismic inversion, which minimizes tuning effects or takes tuning effects into consideration, appears to provide a more robust approach for characterizing thin-bed reservoirs and is the subject of ongoing research (Salleh et al., 1999; Torres-Verdin et al., 1999; Riel, 2000; Pennington, 2001).

Two approaches can be used to recover acoustic impedance from seismic data. One technique is to remove or minimize the tuning effects by applying a deconvolution model. This is the conventional deterministic seismic inversion. A few theoretical methods have emerged since 1980s, such as linear programming (Taylor et al., 1979; Cooke, et al., 1983; Oldenburg et al., 1983), maximum-likelihood or sparse spike inversion (Chi, et al., 1984), and more recently, constrained sparse spike inversion (Debeye et al., 1990). All these methods resolve the components of the convolutional model in each seismic trace.

The other alternative is to not remove the tuning effects from a seismic data set, but to establish a statistical relationship between seismic attributes and impedance (multi-attributes analysis, Hampson et al., 2001). Non-linear statistics are used to describe the relationship between attributes and acoustic impedance as the relation between them is complicated. Neural networks are most often used to construct this non-linear relation. A couple of neural networks that have 
been in use for seismic inversion are the multi-layer feed-forward neural network (MLFN) (Liu, et al., 1998) and probabilistic neural network (PNN) (Hampson, et al., 2001). Non-linear relationships imply that more than one step or expression is required to simulate the observations from the known inputs, in contrast to one step in linear relationship. Each layer consists of nodes and weights. The nodes are connected with weights and the neural network process finds the optimal weights between the nodes by training a data set. Then, the statistical model is applied to simulate an entire data set.

Our study area is again the Boonsville Field, Fort Worth Basin of the north central Texas. In this study, we examine the conventional thin-bed tuning model, apply a new generalized regression neural network model, and perform a comparison study with two other inversion models with a focus on the prediction the thin-bed reservoirs.

\section{Evaluation of the Conventional Thin-Bed Tuning Model}

The conventional thin-bed tuning model was derived from a wedge model. Widess (1973) demonstrated the interaction of the top and base reflectors of a thin bed wedge, illustrating that amplitude increases as thin-bed thickness increases until the amplitude of the composite wavelet reaches a maximum for a bed thickness of one-quarter wavelength. Clearly, the bed thickness at which these phenomena occur depends on the shape of the wavelet or its frequency band and the velocity of the reservoir of interest. For instance, one-quarter of the wavelength is approximately 50 feet for the Boonsville Field data set, given $12000 \mathrm{ft} / \mathrm{s}$ as the average velocity of the Caddo sequence and $57 \mathrm{~Hz}$ as the dominant frequency of the seismic data (Figure 41). It is assumed that the post-stacked (summed) amplitude represents the zero-offset amplitude; that is, AVO effects were ignored.

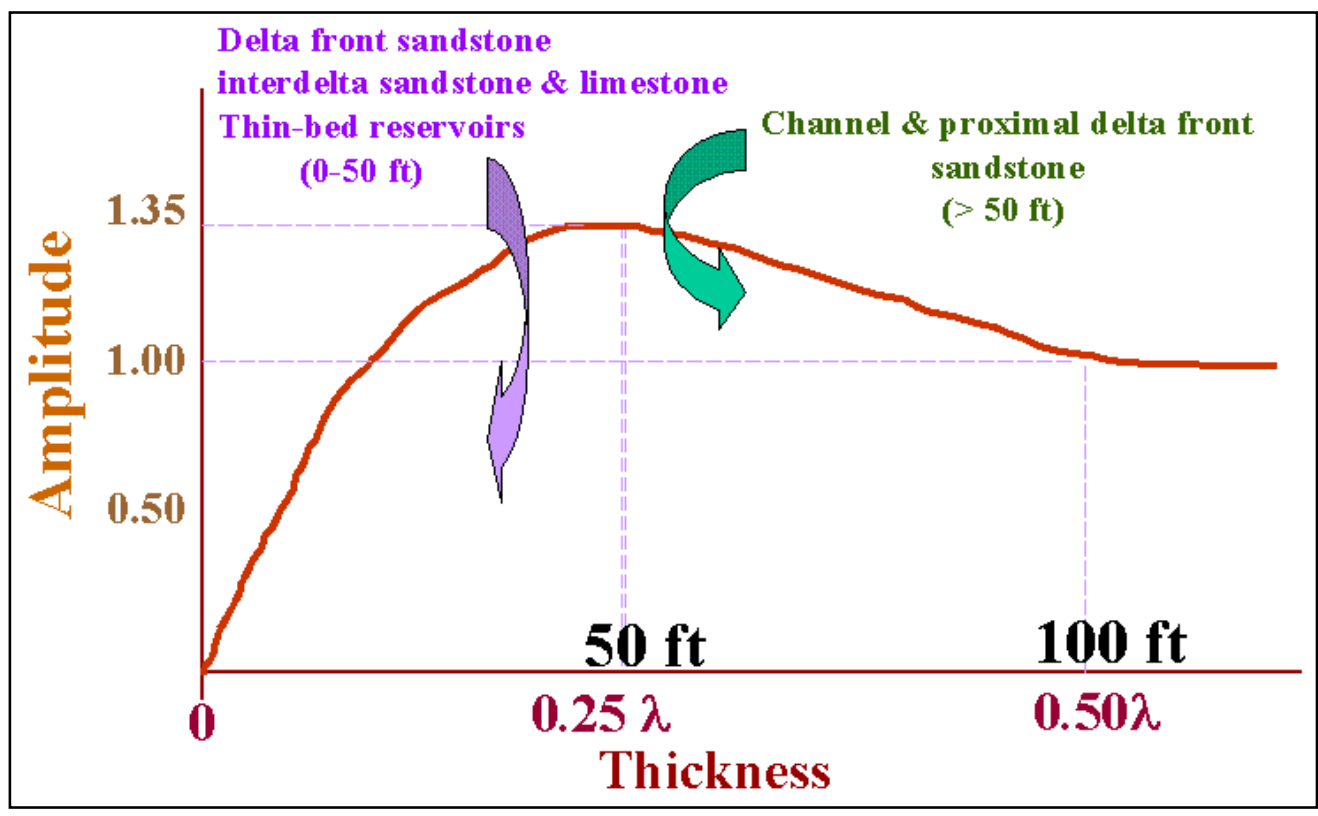

Figure 41:

Conventional thin-bed tuning model with notations for our data set.

The thin-bed tuning model has been widely employed to predict thin-bed reservoirs. A number of case studies have been published during the last decade (Brown, 1996; Hoover et al., 1999; Cooke et al., 1999). In reality, however, a sequence of interest is rarely developed by a single reservoir layer or by multiple widely-spaced reservoir layers. Closely spaced thin-beds will present a challenge in predicting reservoir distribution using the model derived from a single 
wedge model. Notice that multiple thin beds are closely spaced if the vertical distance between two thin beds is less than one-quarter of a wavelength. Therefore, two or more closely-spaced thin beds will destructively interfere, and thus will not obey the simple relationship between amplitude attribute and reservoir thickness developed for a single thin bed.

Two-dimensional (more precisely 1.5-D) seismic forward modeling was implemented to better illustrate the multiple closely spaced model. The wavelet applied in the modeling is a zero-phase Richer wavelet with the same dominant frequency of $57 \mathrm{~Hz}$ as that from the seismic data. The velocities of the sandstone, limestone and shale, based on the statistical results from the acoustic well $\operatorname{logs}$, are 14200, 15200, and $11800 \mathrm{ft} / \mathrm{s}$, respectively. Amplitude attenuation demonstrates the destructive interference between the two closely spaced thin sandstones in a shale-rich sequence (Figure 42).

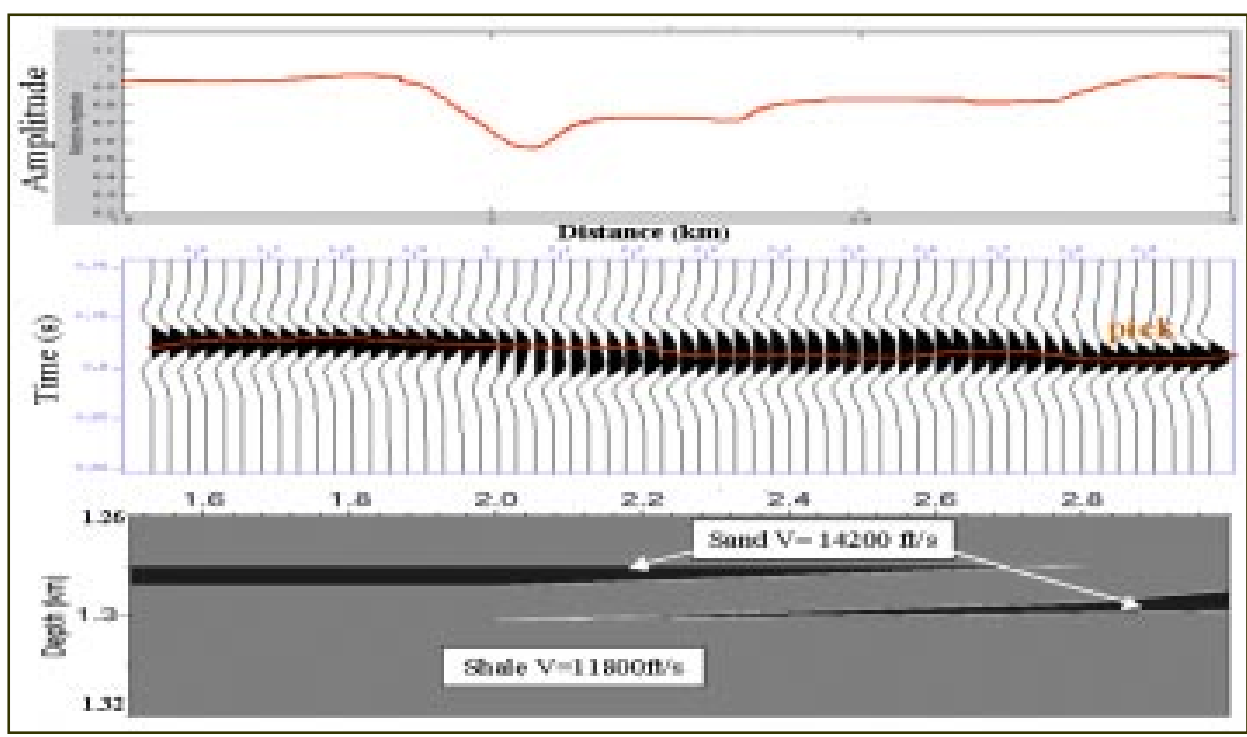

Figure 42: Modeled seismic response showing amplitude attenuation where two closely-spaced thin sandstone layers are developed.

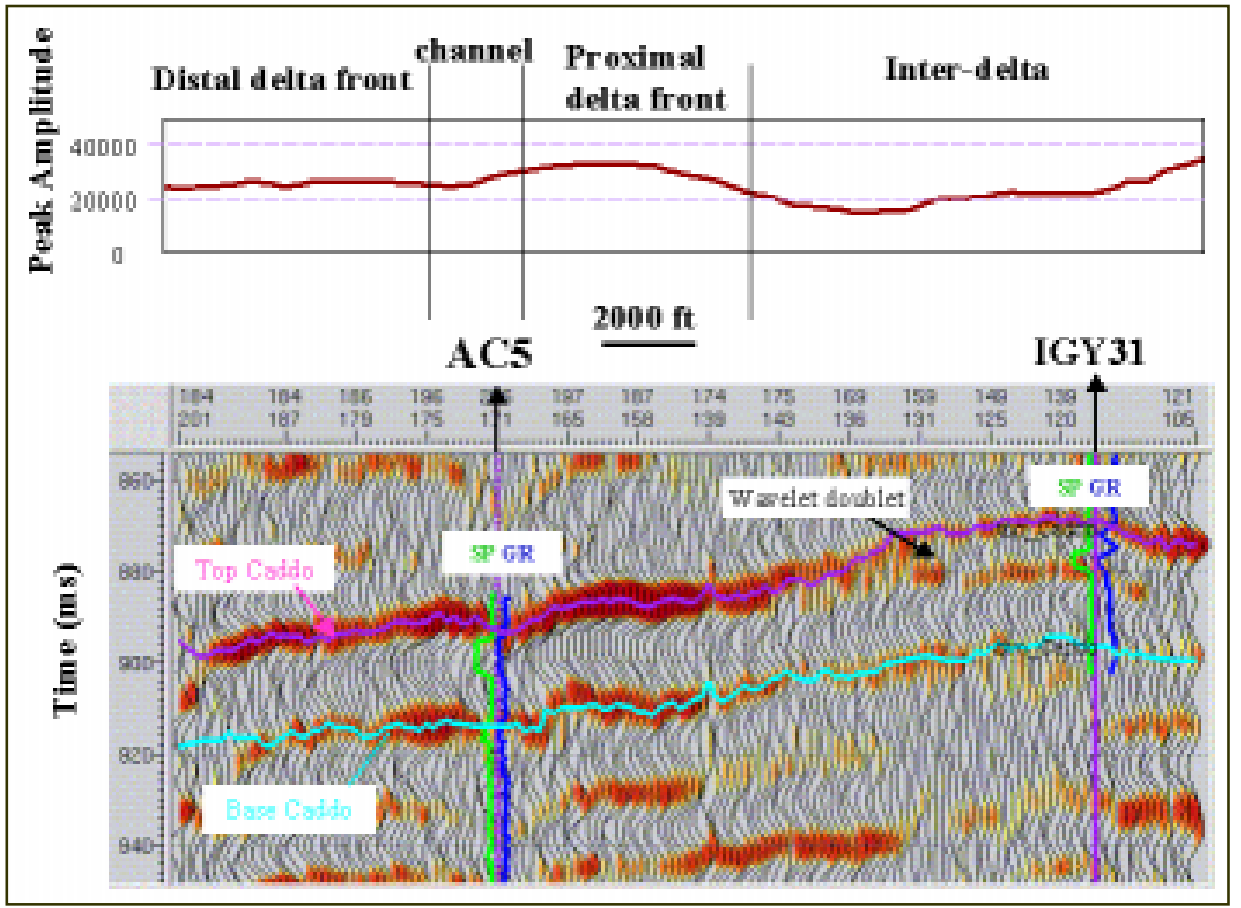

Figure 43: Seismic features through the main delta system showing amplitude decrease and wavelet doublet in interdelta subfacies. 
A seismic traverse line through the main delta system of this study area (oil producing area) is shown in Figure 43. The amplitude attenuates in the inter-delta subfacies where the thin limestone and sandstone are closely spaced. To avoid using biased amplitudes due to a possible peak mistracking, the RMS amplitude computed over $20 \mathrm{~ms}$ time window below the top Caddo horizon was used instead of single point amplitude. The relationship between RMS amplitude and the summed thickness of the sandstone and limestone at 37 wells is shown in Figure 44a. The summed thickness of two types of rock was used because the sandstones cannot be separated from the limestones utilizing the amplitude and/or acoustic impedance alone. It clearly does not agree with the conventional thin-bed tuning model and should not be used for approximating the thickness of sandstone and limestone.
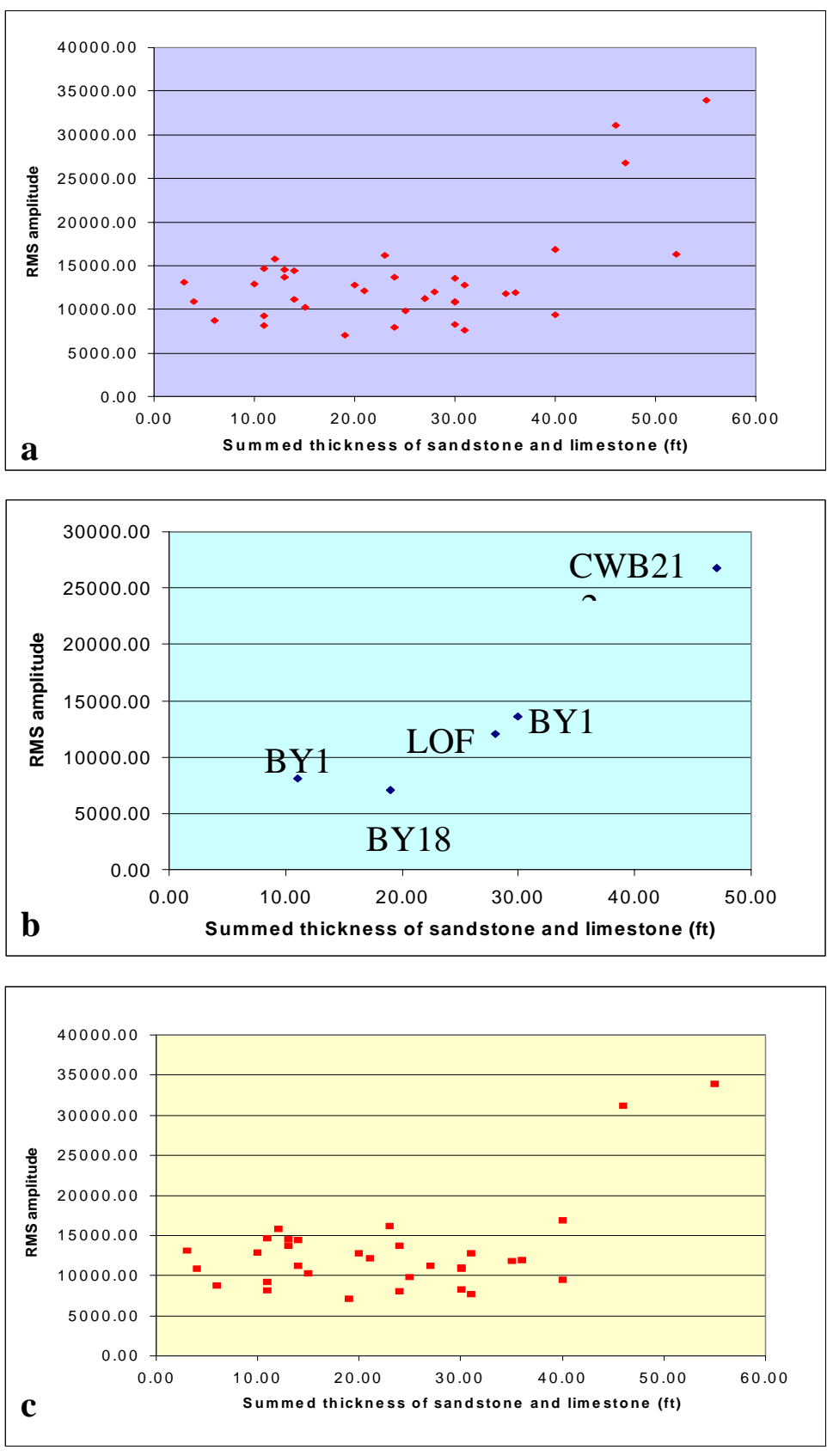

Figure 44: RMS Amplitude plotted against summed thickness of sandstone and limestone.

(a) for 37 wells

(b) for a single thin bed or multiple widely-spaced thin beds

(c) for multiple closely-spaced thin beds.

By interpreting the sandstone and limestone layers and the shale thickness between them, two groups were categorized for this data set: multiple closely-spaced layers and single layer or widely spaced layers. The single layer and widely spaced layers are consistent with the thin-bed tuning model because there is no significant destructive interference between multiple thin beds. The correlation of the amplitude of the second group against the summed thickness of layers is shown in Figure 44b. This suggests that the amplitude increases with increasing thickness at least up to one-quarter wavelet length (50 feet). Therefore, the thin-bed tuning model works for single layer or widely spaced layers (Figure 44b), but fails for the closely spaced thin beds (Figure 44c).

In general, the conventional thin-bed tuning model does not often apply because a single reservoir and/or 
multiple widely spaced reservoirs are rarely developed in a sequence. As a consequence, seismic inversion, which shows more promise in predicting reservoirs, will be investigated in this chapter.

\section{Generalized Regression Neural Network InVErsion}

The prior study showed that the conventional thin-bed tuning model is not applicable due to destructive interference among multiple closely-spaced layers. Here seismic inversion models are employed to derive layer properties (acoustic impedance in this paper). One of our research goals is to use a novel non-linear generalized regression neural network (GRNN) inversion model to predict the thin-bed reservoirs. The results will be compared with two existing inversion software models, that is, the probabilistic neural network (PNN) and constrained sparse spike model (CSSI).

\section{GRNN inversion model}

In this study, a GRNN inversion algorithm was developed and tested on the Boonsville data set. Chen (1996) described the relevant mathematical model in detail. The GRNN algorithm provides estimates of continuous variables and converges smoothly to the underlying regression curve (in case of 1-D). It features instant learning and always gives a smooth transition from one observed value to another. The GRNN model is primarily derived from the radial basis function (RBF) which is a neural network concentrated on localized basis or potential functions and iterative function approximation. This function requires more neurons than standard feedforward back-propagation networks, but it can be designed in a fraction of the time it takes to train standard feed-forward network such as MLFN. Our GRNN model consists of two layers; the first layer is the hidden RBF and the second layer is a linear regression function.

High-cut or low pass filtering should be applied to cut off the very high frequency component of the impedance from wells in order to "upscale" to the seismic frequency from full band frequency of well data. The neural network technique tries to build a non-linear relationship between seismic attributes and impedance by "forcing" processing to incorporate all the information as possible even if some of the "information" is noise. High-cut filtering smoothes impedance curves and ensures that the relationship is not far beyond the seismic resolution. It also reduces some noise. We applied a $120 \mathrm{~Hz}$ high-cut filter (the maximum frequency component) to the impedance logs. Four basic attributes (amplitude, envelope, instantaneous phase, and instantaneous frequency) were used as an input set to the GRNN neural network. A convolution model was applied to better link these attributes to the acoustic impedance. This convolutional operator is consistent with the geophysical model and adds more variables to the input vector, thus improving the accuracy of the neural network (Hampson et al., 2001).

\section{Application of the GRNN model to the Boonsville Field data set}

This GRNN inversion approach was applied to the Boonsville Field data set and six wells (AC5, BY11, BY13, BY18D, CY9, and IGY31) were used for the training data set because their synthetic curves can be tied to the seismic traces without "squeezing and stretching". The correlation coefficient between inverted and actual impedance at the wells in the training set is 0.84. A blind test was conducted in which one well at a time was removed from the training set; the inversion result at the associated seismic trace was compared with the impedance from that 
well, which obtains coefficient of 0.52 . The inverted impedance can be used to predict the presence of the sandstone reservoirs by interpreting high impedances of $32000 \mathrm{ft} / \mathrm{s} * \mathrm{~g} / \mathrm{cc}$ as a base value (Figure 45). The impedances of the sandstone reservoirs and limestones are greater than the base value whereas the impedance of the embedding shale is less than the base value.
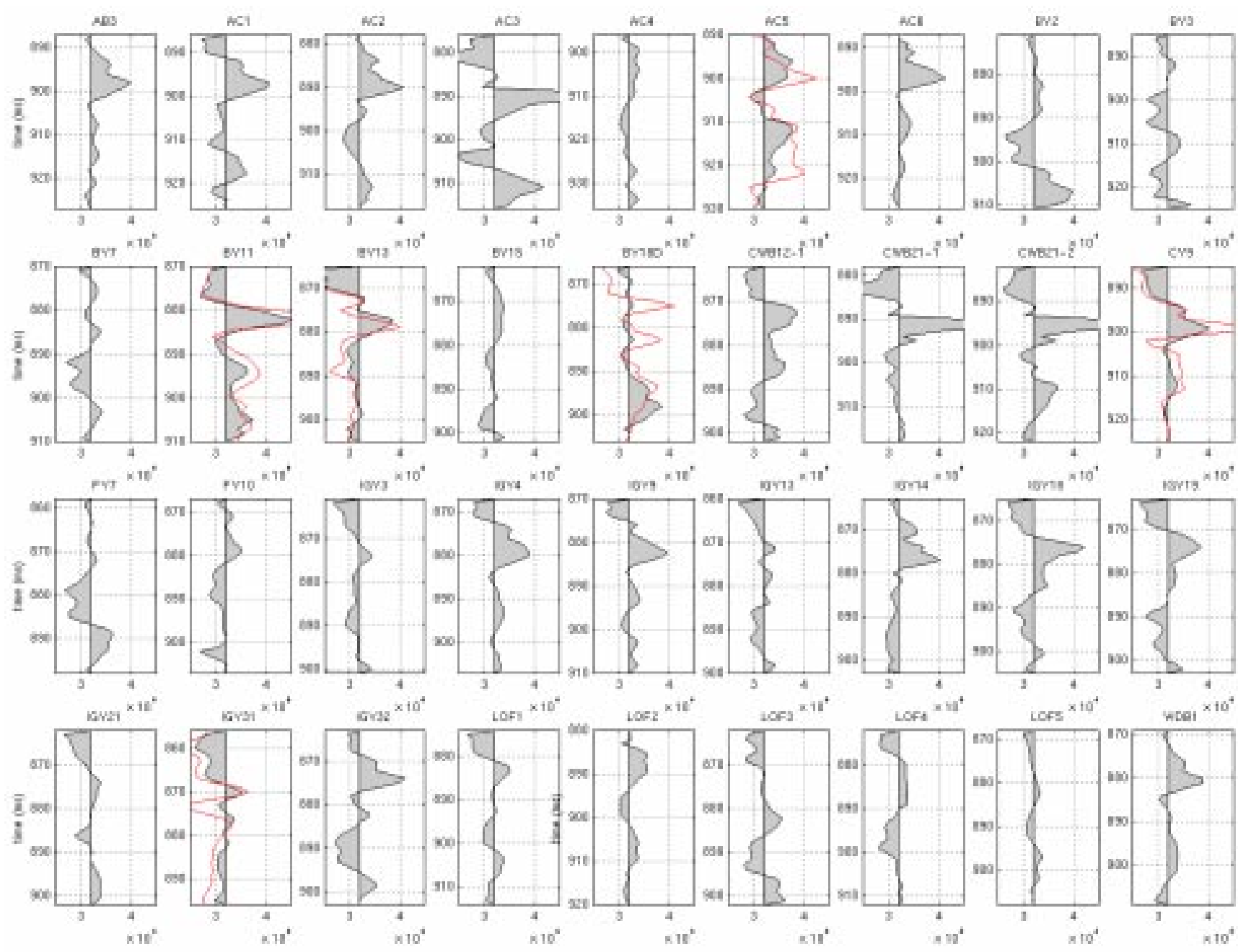

Figure 45: Inverted impedance curves using the GRNN model at 36 wells.

The base line represents $32000 \mathrm{ft} / \mathrm{s} * \mathrm{~g} / \mathrm{cc}$, and the original acoustic impedance curves are shown at the six wells for which they could be computed, in red.

Our GRNN inversion made correct predictions at all well locations in the proximal delta front and distal channel (wells AC3, CWB21-1, CWB21-2, and AC5) where thicker sandstones were developed and inverted impedance is more than $39000 \mathrm{ft} / \mathrm{s} * \mathrm{~g} / \mathrm{cc}$. The inverted impedance at well $\mathrm{AC} 4$ is around $32000 \mathrm{ft} / \mathrm{s}^{*} \mathrm{~g} / \mathrm{cc}$ and consistent with the fact that no reservoir and only thin shaly limestones were deposited. At well BY2 and BY3 where only sandy shales or shaly sandstones are present, the inverted impedance is lower than or near to $33000 \mathrm{ft} / \mathrm{s} * \mathrm{~g} / \mathrm{cc}$. The inverted impedance at the well FY10 ranges from 33000 to $34000 \mathrm{ft} / \mathrm{s}^{*} \mathrm{~g} / \mathrm{cc}$ and agrees with the presence of the shaly sandstones at that transition interval from the delta front to prodelta subfacies. At well FY7, sandy shale was found and its inverted impedance is lower than the base value. All of 
these show that the inverted impedance was able to identify the presence of the thicker sandstones and limestones.

Twenty wells out of the 30 blindly tested wells are located in the distal delta front and interdelta subfacies (AB3, AC1 AC2, AC6, WDB1, LOF1, LOF2, LOF3, LOF4, LOF5, CWB12-1, IGY3, IGY4, IGY9, IGY13, IGY14, IGY18, IGY19, IGY21, and IGY32, see Figure 45). The GRNN approach was able to detect the thin-bed sandstone and/or limestone at 13 out of 20 wells. The model was not able to identify the thin-bed sandstones and limestones at 7 wells in the distal delta front (LOF1, LOF3, LOF4, LOF5, IGY3, IGY13, and IGY21). The high impedance layers (limestones) in the prodelta subfacies were correctly predicted at wells BY11 and BY13, but not at wells BY18D and BY15.

Two inverted impedance cross sections (crossline 125 and 171) are depicted in Figure 46. The high impedance is highlighted in the east, center and west, indicating the main delta sandstone reservoirs, minor distributary channel sandstone, and limestone, accordingly. Notice that the inverted impedance is relatively low at some traces in the center of the crossline 171 (the "doublet wavelet" region). The impedance cube with different opacity cutoffs is shown in Figure 45 which highlights the thicker sandstone in the east (proximal delta front) and the thicker limestone in the west (prodelta subfacies), minor distributary channel sandstone in the center, thin beds in the east and south, and distal delta front sandstones and/or limestones in the east. The inversion technique also easily reveals the lateral discontinuity of the sandstone reservoirs and limestones in the Caddo sequence.

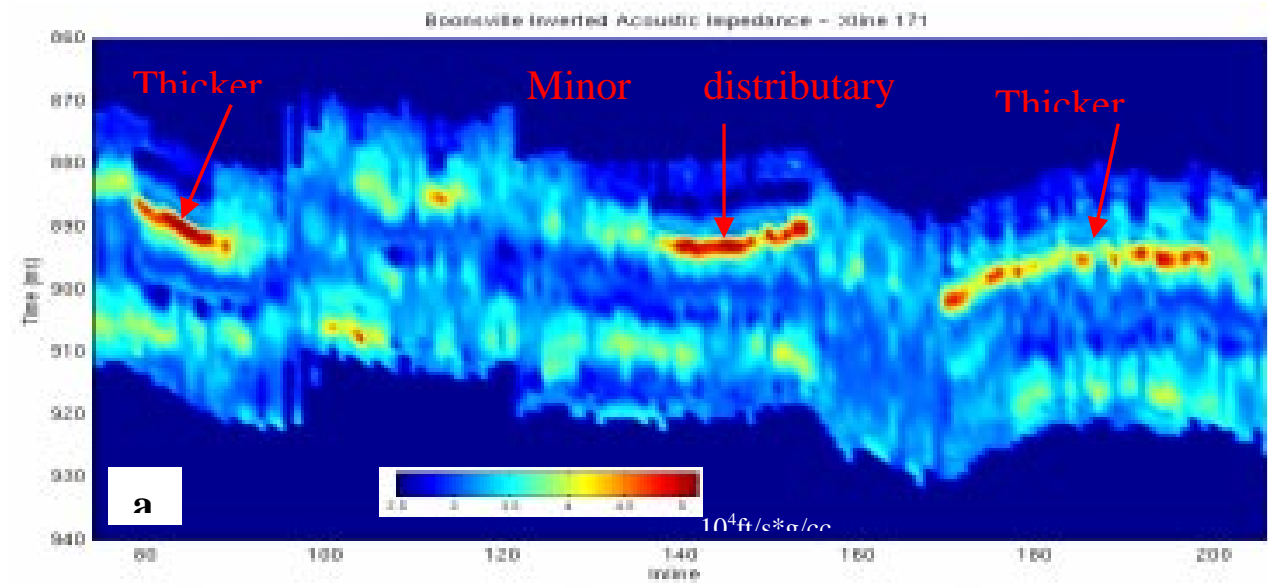

Figure 46:

Crosslines 171

(a) and 125 (b) of

the inverted

impedance data

using the GRNN

model indicating

sandstone and

limestone as

higher

impedances.

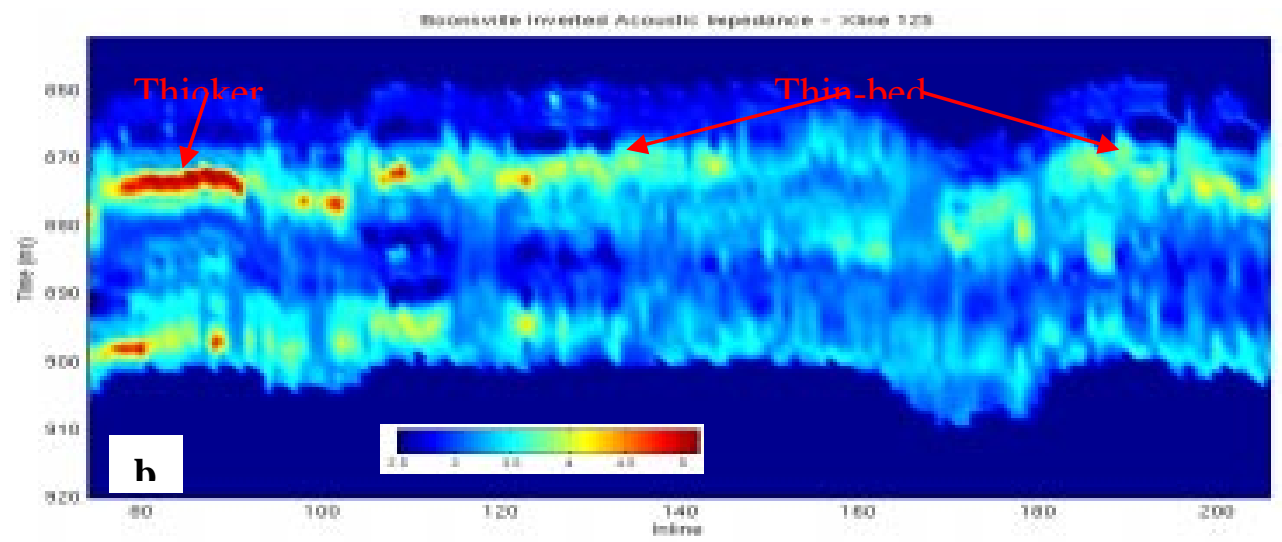

Final Technical Report 


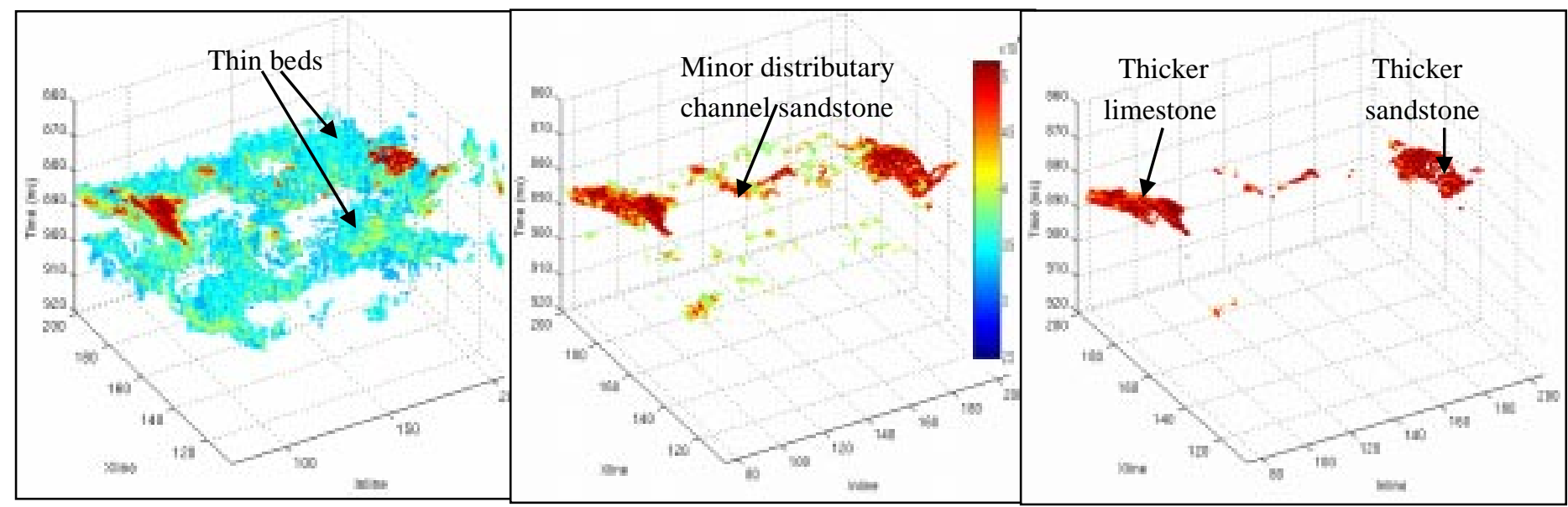

Figure 47: Inverted impedance volume (GRNN model) with different opacity cut-offs.

Left: $32000 \mathrm{ft} / \mathrm{s} * \mathrm{~g} / \mathrm{cc} \quad$ Center: $36000 \mathrm{ft} / \mathrm{s}^{*} \mathrm{~g} / \mathrm{cc} \quad$ Right: $39000 \mathrm{ft} / \mathrm{s}^{*} \mathrm{~g} / \mathrm{cc}$

\section{OTHER INVERSION MODELS}

\section{Probabilistic neural network inversion (PNN)}

The PNN inversion is a two-layer neural network with the hidden layer as its first layer. According to Hampson et al. (2001), this hidden layer might be similar to the RBF in the previous GRNN model because the Gaussian basis function is used in this layer to compute the distances from the input vector to the training vector (Hampson et al., 2001). However, the second layer is different in that it utilizes a compete transfer function based on the probabilities, instead of a regression function in the second layer of the GRNN model.

The PNN inversion proceeded in the following steps: (1) wells were tied to seismic traces, (2) training and validating were preformed on the nine pre-selected wells (AC5, AC6, BY11, BY13, BY18D, CY9, IGY14, IGY19, and IGY31) in an effort to establish a neural network relationship between the seismic attributes and actual acoustic impedance, and (3) the inverted impedance data was analyzed. The training results with correlation coefficient of the inverted and actual impedance is 0.86 , with a validation coefficient of 0.56 , implying the prediction accuracy is about 0.56 . This prediction accuracy is close to our GRNN model of 0.52 .

Figure 48 shows the impedance slice $6 \mathrm{~ms}$ below the top Caddo horizon and demonstrates the main geological features of the Caddo sequence. The highest impedance areas (in red) in the east and west represent the thicker sandstone reservoirs and limestones, respectively. A northwest-oriented zone with the inverted impedance of 33000-35000 indicates the minor distributary channel (no well was drilled yet). In the east and south, the intermediate impedance, ranging from 32000 to $37000 \mathrm{ft} / \mathrm{s}^{*} \mathrm{~g} / \mathrm{cc}$, indicates the presence of the thin-bed sandstone and/or thin-bed limestone, corresponding to the distal delta front and interdelta subfacies. The inverted impedance is slightly lower than the actual impedance in those regions because of the truncation related to the scale problem; however, it is still higher than the inverted impedance in the northcentral parts where no clean sandstone and pure limestone were deposited. Notice that some isolated thin-bed limestones in the prodelta were not predicted, for instance, at well BY15. 


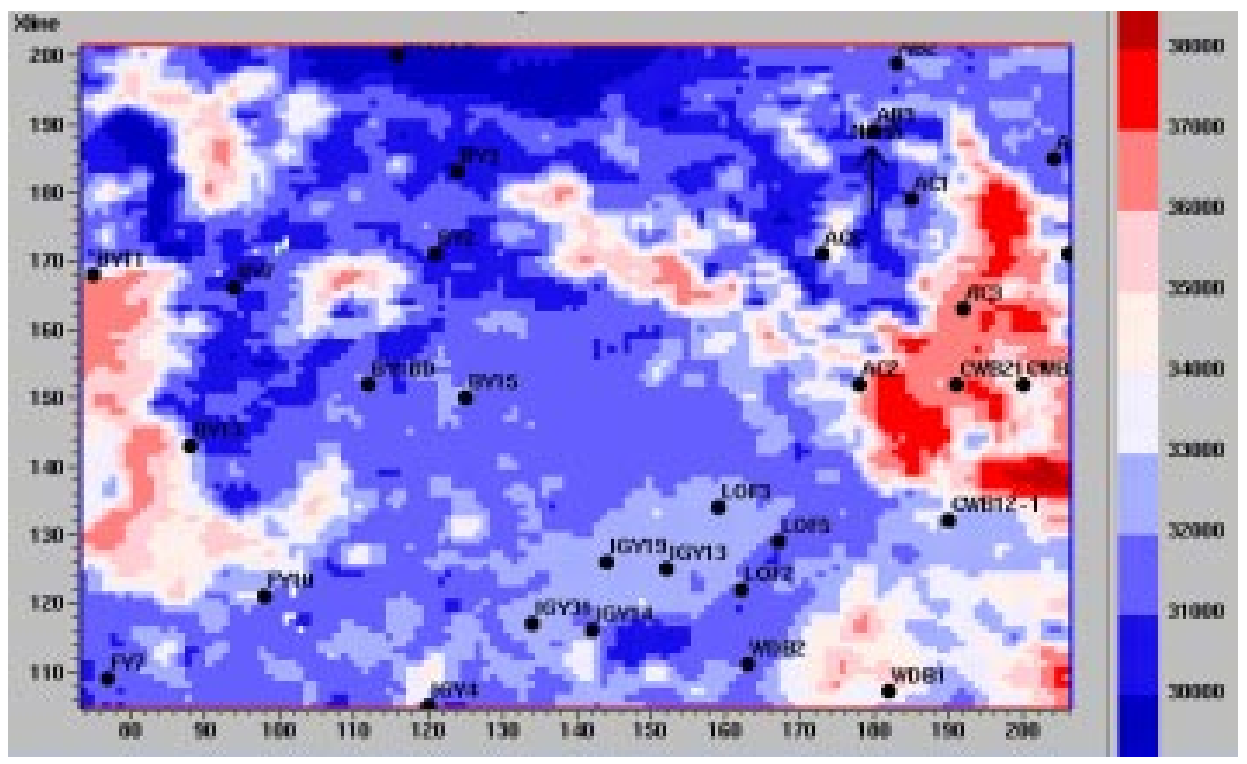

Figure 48: Inverted impedance data using the PNN model.

Time slice $6 \mathrm{~ms}$ below the top Caddo horizon, showing the distribution of high impedances.

One representative crossline (117) is shown in Figure 49. Notice that high impedance, ranging from 37000 to $45000 \mathrm{ft} / \mathrm{s} * \mathrm{~g} / \mathrm{cc}$, indicates the thicker sandstone reservoirs and/or limestone nonreservoirs and the intermediate impedance from 33000 to $37000 \mathrm{ft} / \mathrm{s}^{*} \mathrm{~g} / \mathrm{cc}$ represents the thin-bed sandstone reservoirs and/or thin-bed limestone non-reservoirs because of the truncation. Crossline 117 illustrates the high impedance layers in the east and center. They represent the thin-bed sandstone reservoirs and the closely spaced thin-bed sandstone reservoirs, respectively (compare with the well-based depositional model, Figure 35). The thicker limestones in the west were not revealed in crossline 117 while the GRNN did recognize them (Figure 46). This also demonstrates that the sandstones and limestones are heterogeneous.

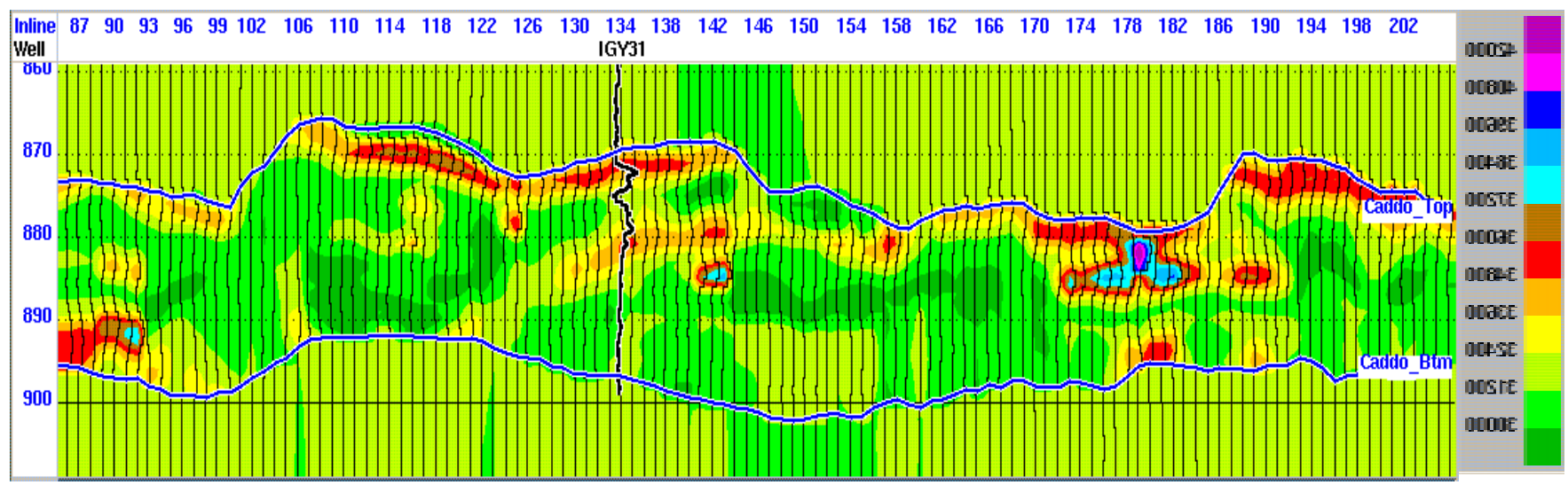

Figure 49: Crossline 117 of the invertedimpedance volume using the PNN model.

It is difficult to discriminate the sandstone from the limestone using the inverted impedance alone. However, they can be separated by knowing the depositional model already constructed using well and seismic data (see previous section in this report). In order to interpret the limestone and sandstone from the impedance cube, the cutoff of $32000 \mathrm{ft} / \mathrm{s} * \mathrm{~g} / \mathrm{cc}$ was obtained on the basis of well data. The thicknesses from 37 wells are shown in Figure 50, against the 
thickness estimated from the inversion results. This relationship demonstrates the advantage of using impedance or layer properties over using the amplitude attribute for thin bed estimates (see Figure 44a). The estimated sandstone reservoir distribution is illustrated in Figure 51. Note that the limestones in the west were manually removed, but we was not able to separate them from the sandstones in the interdelta (i.e. in the south) and western margin of the main delta system. This figure shows that no reservoir sandstone was developed in some parts of the main delta system, supporting the lateral heterogeneity of the thin-bed reservoirs in the shale-rich Caddo sequence.
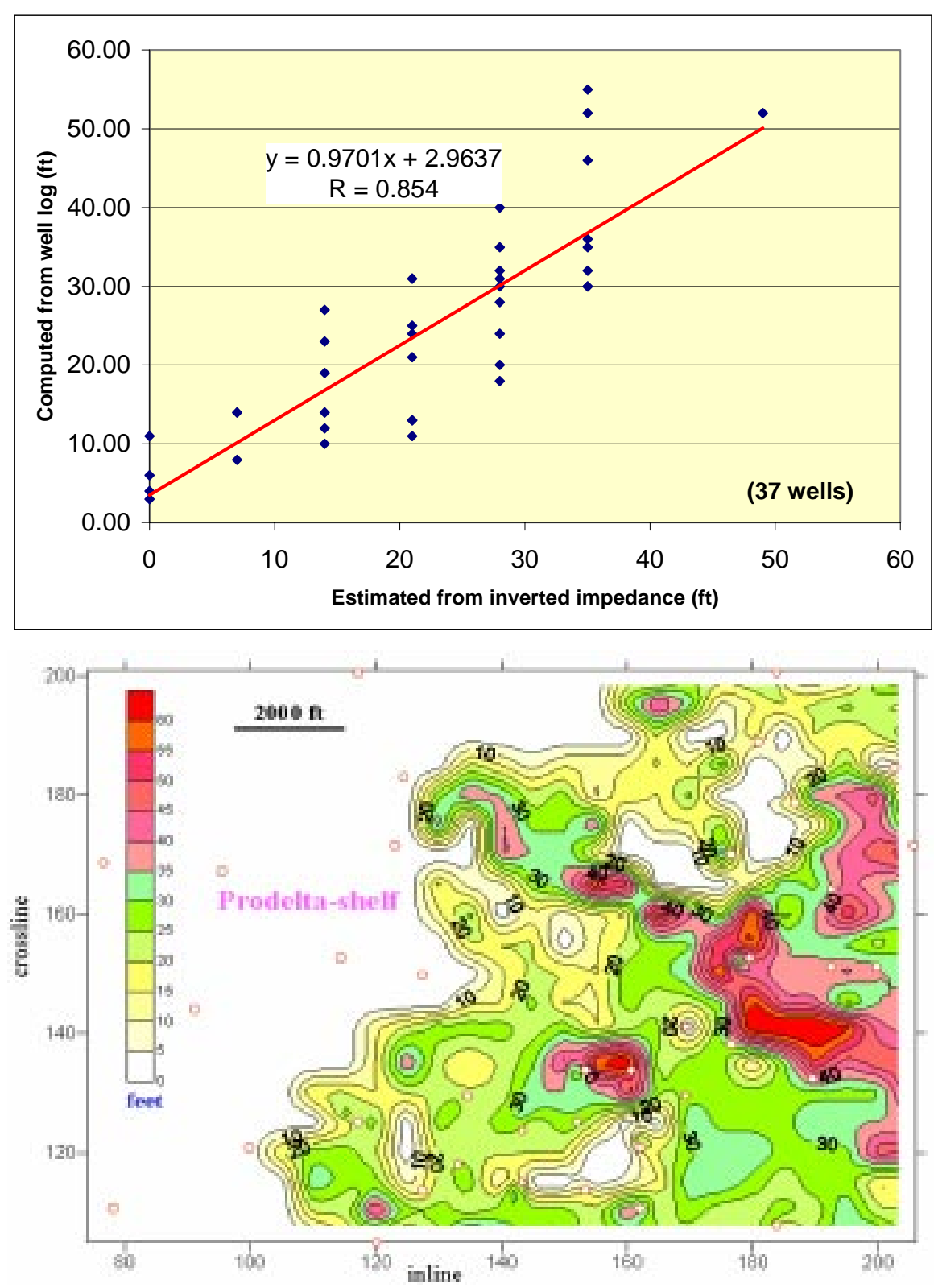

Figure 50: Actual sandstone and limestone thickness plotted against predicted thickness from seismic inversion (PNN model)
Figure 51: Estimated sandstone thickness of Caddo main delta system (PNN model)

Note: the limestones in the west were removed manually in this example) 


\section{CONSTRAINED SPARSE SPIKE INVERSION (CSSI)}

The CSSI inversion model is based on the convolution and deconvolution model. It involves two fundamental techniques: wavelet estimation and balanced spike and wavelet deconvolution (Chi, et al., 1984; Yilmaz, 1987; Russell, 1988; Debeye et al., 1990). The CSSI inversion was applied to the Boonsville data set. Six main procedures were followed in performing the CSSI inversion, with a focus on the quality control: (1) building earth model, (2) estimating wavelets based on 5 wells (BY11, BY18D, CY9, AC5, and IGY31), (3) conducting constraints, (4) inverting trace by trace, (5) merging traces, and (6) interpreting inverted impedance data. The inverted impedance agrees with the actual impedance with the correlation coefficient of 0.72 for the Caddo sequence (Figure 52).

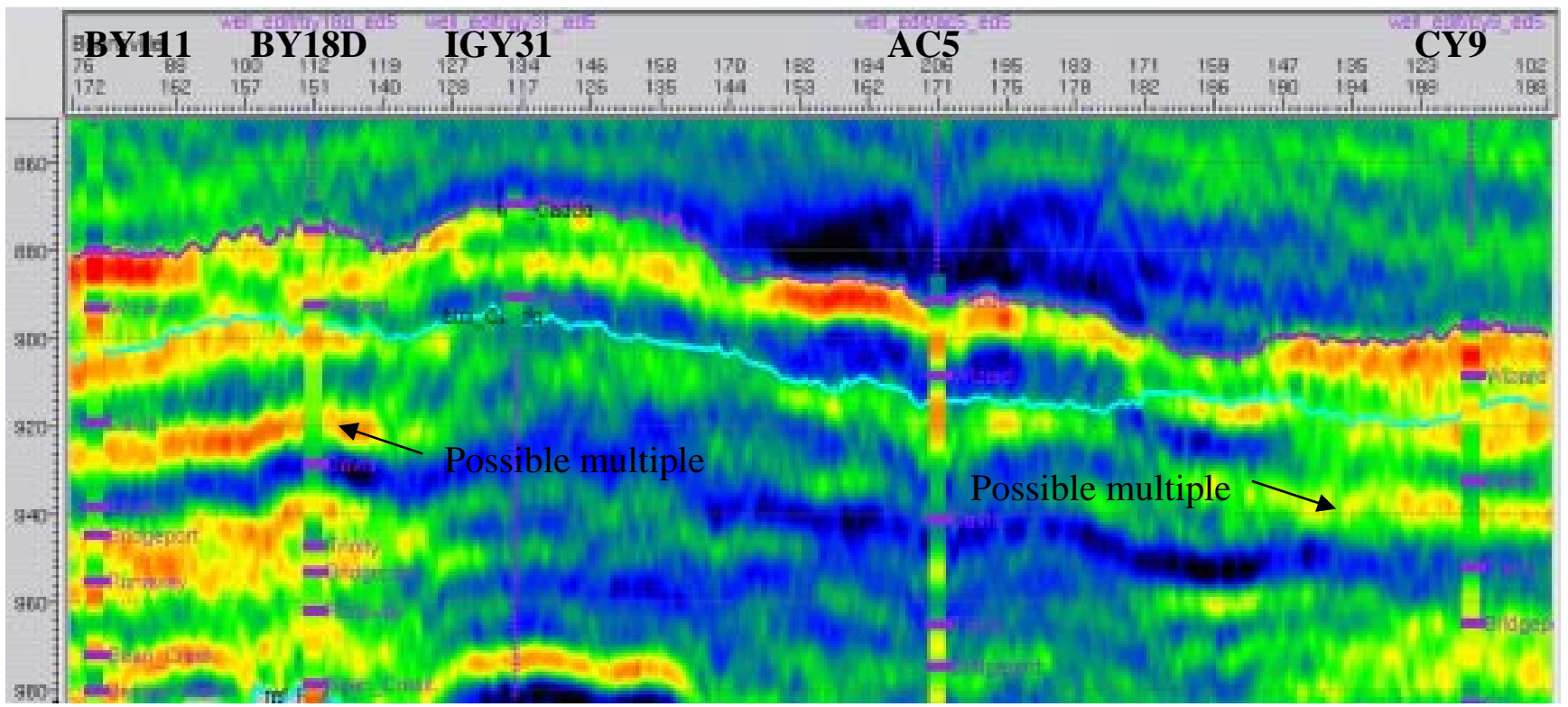

Figure 52: Traverse line showing the inverted impedance using CSSI model and agreement with well data (the five wells indicated were used to estimate the wavelet and provide constraints).

A high quality match between inverted and actual impedance layers would allow us to estimate the sandstone and limestone by tracking the high impedance layers. However, the layers with the impedance higher than $33000 \mathrm{ft} / \mathrm{s}^{*} \mathrm{~g} / \mathrm{cc}$ in the Caddo sequence were found in the entire survey including the prodelta subfacies (Figure 36, top). Furthermore, the inverted impedance in most of the distal delta front and inter-delta subfacies is the lowest, which prevents us from identifying the thin-bed sandstones and limestones. For instance, the inverted impedance at well BY3 and BY3, where no sandstone and limestone were interpreted, is not lower than the impedance in the delta front area. A variety of opacity cut-offs was explored as shown in Figure 53, which reveals that thicker sandstone and/or limestone were able to be delineated (Figure 53, bottom). The CSSI inversion provided few indications of the thin-bed reservoir distribution. The parameter $\lambda$ may be varied in order to obtain more thin-bed results, but at the risk of inducing additional noise. 


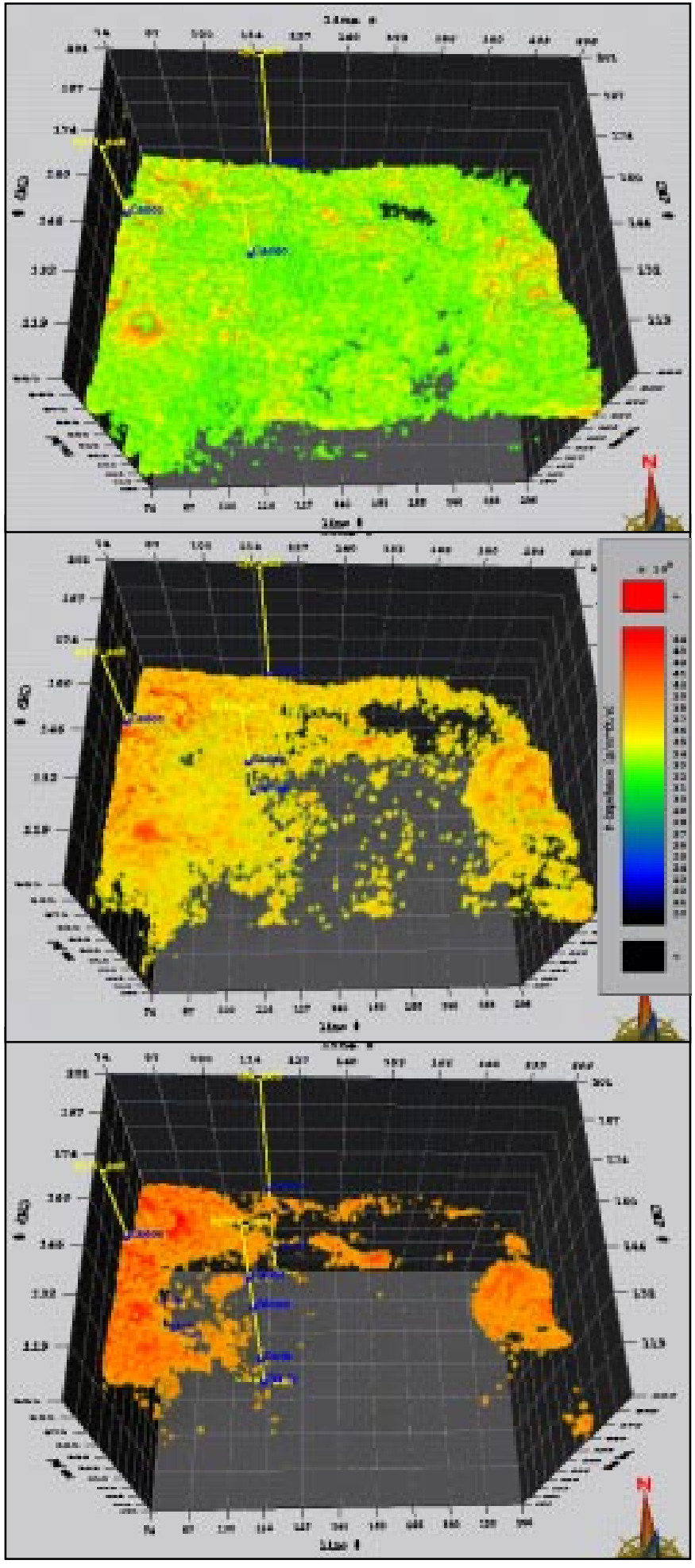

Figure 53: Inverted impedance volume using the CSSI with different opacity cutoffs.

\section{Top: $33000 \mathrm{ft} / \mathrm{s}^{*} \mathrm{~g} / \mathrm{cc}$}

Center: $36000 \mathrm{ft} / \mathrm{s}^{*} \mathrm{~g} / \mathrm{cc}$

Bottom: $39000 \mathrm{ft} / \mathrm{s} * \mathrm{~g} / \mathrm{cc}$

\section{CONCLUSIONS}

Evaluation of the conventional thin-bed tuning model (wedge model) shows that it is appropriate only if one single thinbed is developed or multiple widely spaced thin beds (greater than one quarter of the wavelet length) are present in the sequence of interest. In other words, the model should not be applied for multiple closely spaced thin-beds because of significant destructive interference.

All three inversion models were able to identify the thicker reservoir sandstones and non-reservoir limestones. However, the resulting details for the thin-beds vary. The Boonsville case study shows that our GRNN inversion detected the thin-bed sandstone and limestone at the rate of 13 out of 20 wells. The PNN inversion was also able to predict most of the thin-beds and enhanced the thickness estimates of the thin-bed sandstone and limestone, but the inverted impedance is nosier than that using the GRNN model. The deterministic approach with constraints (CSSI) shows a good agreement of the inverted impedance with the actual impedance logs as a whole, but provided few indications of the thin-beds, perhaps due to our avoidance of noise. For this post-stack data set, it is implicitly assumed that the stack volume represents zero-offset reflections, an assumption that may cause some systematic errors in the deterministic inversion. 
Non-linear statistical inversion focuses on both amplitude and trace shape in the convolution window. This method always forces the system to construct a relationship between multiple seismic attributes and acoustic impedance. As a result, AVO effects have less influence on it. It is more robust in delineating thin-bed reservoirs where multiple wells are available. However, the statistic inversion methods require a number of wells. The GRNN inversion should be very useful in detecting thin-bed reservoirs in existing fields where a number of wells are available.

\section{DFM ANALYSIS OF BOONSVILLE DATA}

The results of the DFM analysis conducted by TransSeismic International entail an interpretation scheme that is different from that used for Stratton (complete report is in the appendix). In Boonsville, the migration paths used by hydrocarbons are sought, in part because the rocks are harder and the fluids require enhanced fracture paths for transport, and in part because the karsting that occurred in deeper intervals has created these pathways. In TSI's conclusion, the potential productive zones could either be in the regions of decreasing effective pressure or along the boundaries between blocks of decreasing pressure and of increasing pressure. Figure 54 shows the relationship of pressures in the productive Caddo zone with the initial oil productivity of wells in the field, from that zone. Figure 55 shows a three-dimensional view (created at MTU using data provided by TSI) of the decompressing regions, shown in red.

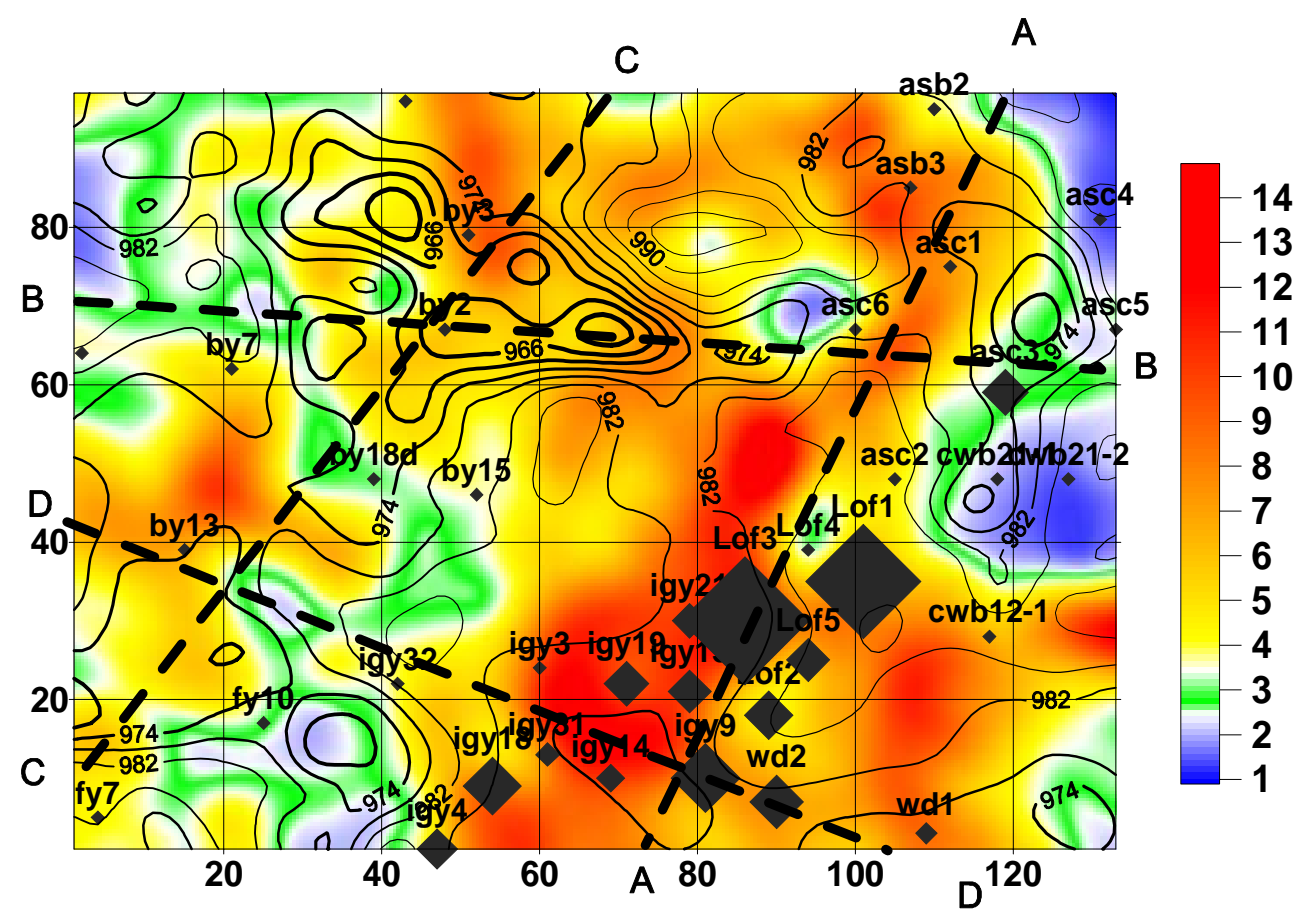

Figure 54: DFM results for the Caddo interval (red represents decompressing regions). The dashed lines indicate block boundaries interpreted by TransSeismic from the data. The size of the diamond symbols is proportional to the oil recovery from the Caddo interval from wells in the field. 


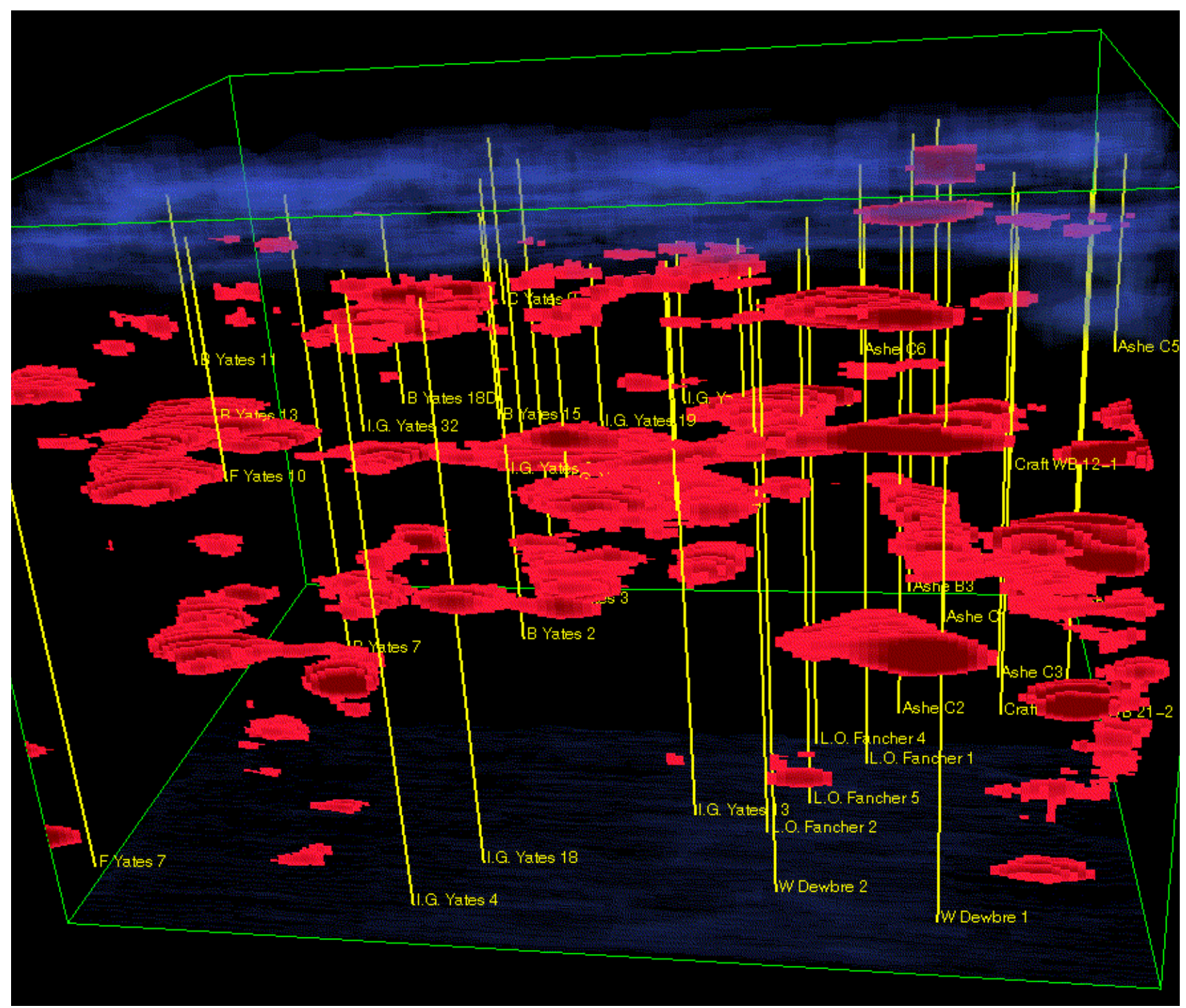

Figure 55: Three-dimensional image of the DFM results for the Boonsville data set, processed by TransSeismic and imaged at MTU; the red colors represent areas of decreasing effective pressure, presumed to have greater potential for hydrocarbon accumulations in the DFM approach. 


\section{Evaluation of seismic attributes in Wamsutter Field, Wyoming.}

Wamsutter field, WY, is operated by BP, and formerly Amoco (see Figure 56 for location). Production here is primarily from the top of the Almond formation with some additional production coming from deeper parts of the Almond in some areas. This field provided a great challenge: conventional attributes were not known to provide any assistance in identifying potential 'sweetspots' or other productive areas. The field is also near the Siberian Ridge field, the subject of a separate, unrelated, DOE/GRI field study which also found that conventional seismic attributes were of little use. There were several reasons for choosing this field for our calibration/test study area. We wanted a site that was would present a challenge to the use of seismic attributes, yet which clearly had wide variation in the production from well to well that was apparently associated with geologic features (and not differences in completion techniques, for example). We also sought a field that presented difficulty in tying seismic data to well $\log$ data due to wide velocity variations within thin beds, and this field contained finely interbedded coals and high-velocity sands in order to examine various aspects of upscaling velocity functions. Ideally, our final field study would also provide a good test case for possible pressure compartments, particularly ones that are associated with fine-scale fracturing; although any pressure compartments that may be present in the Lewis shale above the productive horizons at Wamsutter are not producing oil or gas.

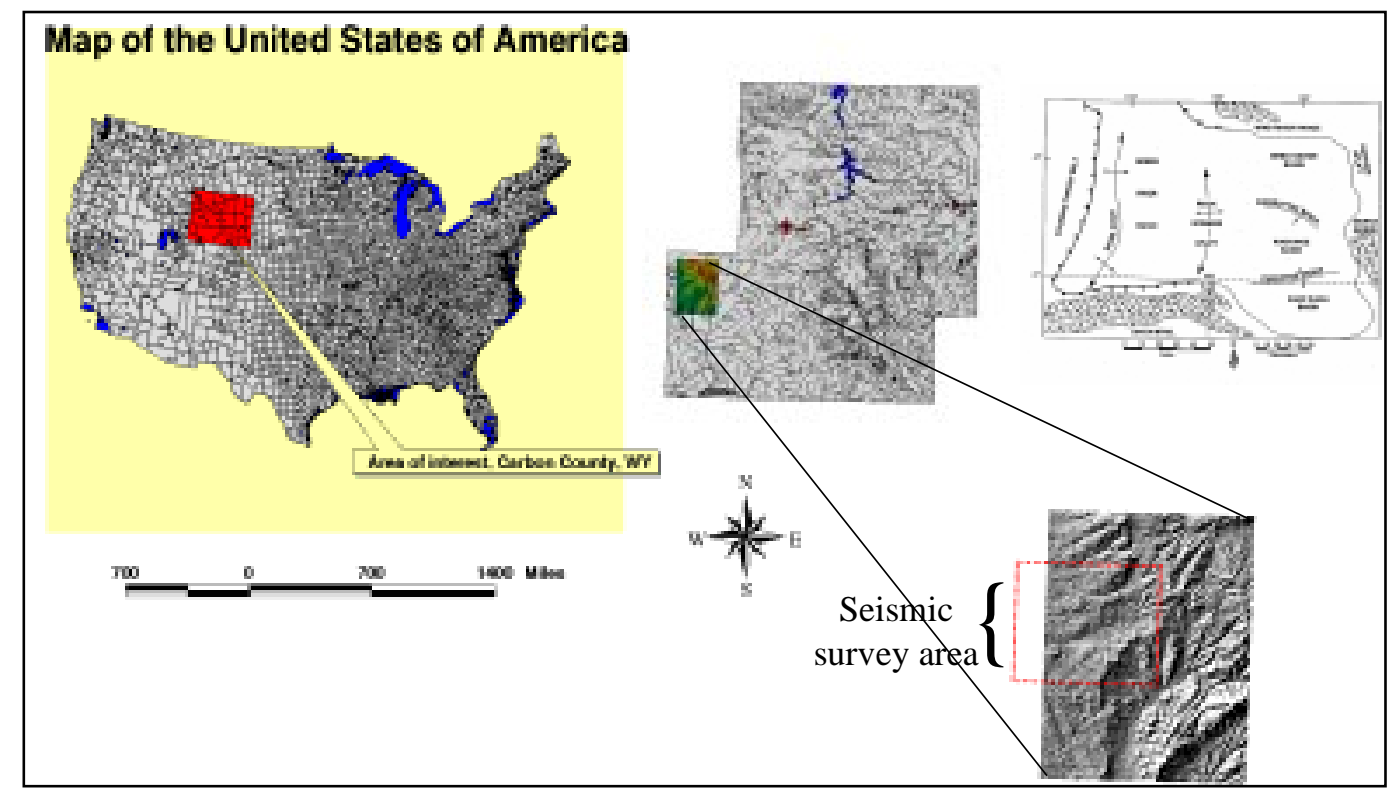

Figure 56. Location maps showing Wamsutter field.

The one piece of data lacking from the field was a vertical seismic profile (VSP) or checkshot, but our upscaling study was completed with some confidence in spite of this shortcoming. Our results demonstrate that the selection of Wamsutter field was appropriate: we have identified unconventional seismic attributes that are convincingly associated with the presence of a sandbar or barrier-island facies that is also highly productive, and other unconventional attributes that may be associated with areas of high fluid pressure and/or dense microfracturing, also areas of higher productivity. 


\section{Location AND GeOlogic SeTting}

The study area, the Wamsutter field, is situated in southwestern Wyoming in the Greater Green River Basin. The area of investigation is in the Rocky Mountain foreland structural province, which is comprised of high desert basins surrounded by breached anticlines with Precambrian cores, and thrust-faults.

Greater Green River Basin contains several geologic structures: the Rock Springs uplift, the Great Divide Basin, the Wamsutter Arch, the Washakie Basin, and the Green River Basin. The Wamsutter Arch separates the Great Divide Basin from Washakie Basin. The Wind River thrust, Thrust Belt, Uinta Mountains, Sierra Madre, and Rawlins uplift define the northwestern, western, southwestern, southeastern, and northwestern margins of the Greater Green River Basin respectively (Figure 57) (Jaworovski and Simon, 1995).

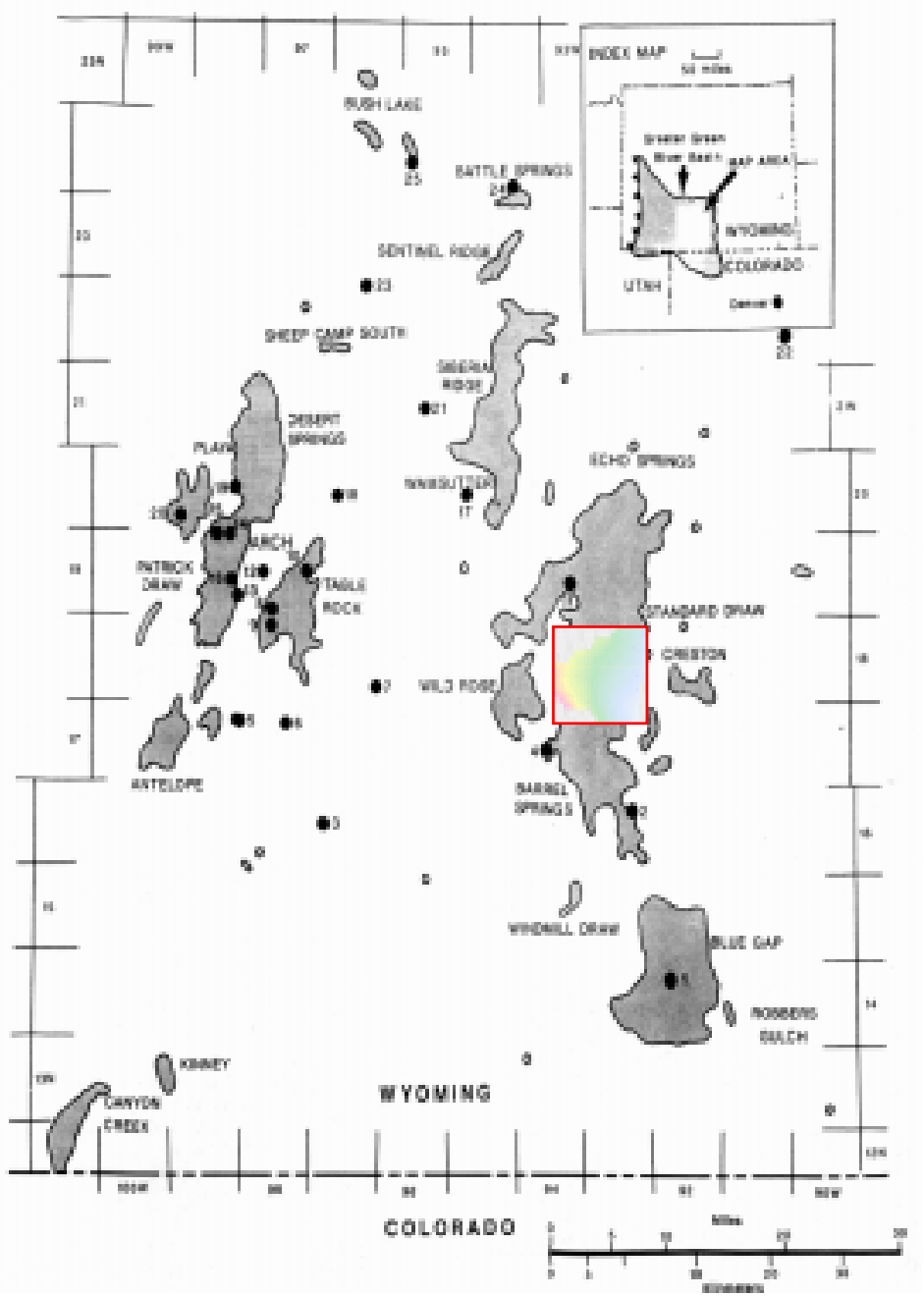

Figure 57: Location of the

Wamsutter survey area. After

Stephenson et al, 1987, and Keighin

et al, 1989.

\section{STRATIGRAPHY}

The main target in Wamsutter field is the Almond Formation. The Almond Formation is the youngest formation (Upper Cretaceous) in the Mesaverde Group of southwestern Wyoming (Figure 58). In the subsurface, the Almond Formation can be subdivided into three genetic units: an upper unit which is transgressive in origin and which includes a highly productive barrier-bar facies and a coal-bearing facies deposited dominantly in back-barrier environments; middle unit, consisting of several dominantly marine shaleto-sandstone sequences that contain little or no coal and a lower, fluvially dominated, coal-bearing unit. The upper Almond is the primary exploration target, significant gas potential may also exist in the middle and lower Almond, as it contains numerous thick coals, which have generated both oil and gas and have expelled significant quantities of gas. (Martinsen et. al., 1995).

The Almond Formation is overlain by the marine Lewis Shale and underlain by the Ericson Sandstones. The thickness of the Almond Formation in the Washakie and Great Divide Basins varies from 250 to more than $500 \mathrm{ft}$. The drilling depths to the top of Almond Formation vary 
from a few thousand $\mathrm{ft}$ to over $16000 \mathrm{ft}$ in the center of the Washakie Basin (Hendricks, 1994). Variations in the formation thickness and in lithofacies may be due, at least in part, to syndepositional movements along basement block faults. Both its upper contact with the Lewis Shale and its lower contact with Ericson Sandstone stratigraphically rise to the west. The Almond-Ericson contact is commonly very sharp (Fig.58). The contact between the Almond and the Lewis is very sharp and is marked by a transgressive surface of erosion (Martinsen et. al., 1995).

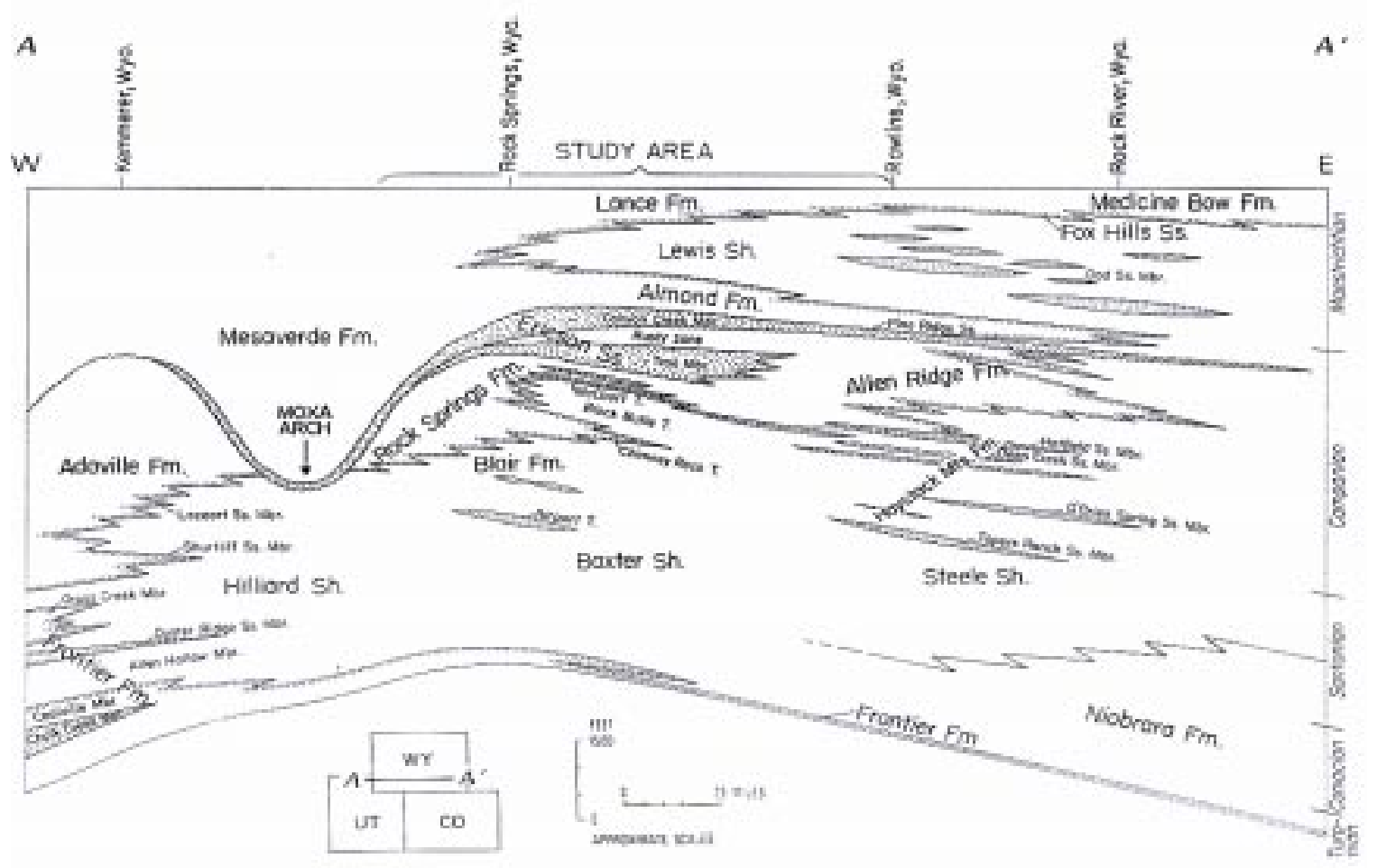

Figure 58: Schematic cross-section (after Roehler, 1990), showing the Wamsutter area of study.

The Almond Formation is one of the most important gas producing units in southwestern Wyoming. Most of the gas production in the eastern areas of the Washakie and Great Divide Basins and intervening Wamsutter arch is predominantly from the marine sandstones of the upper Almond. Within this area approximately 65 fields produce from the Almond Formation.

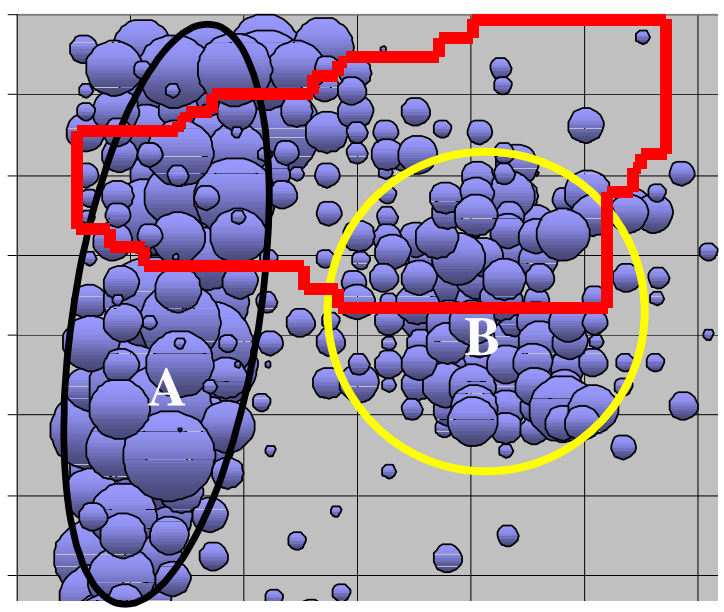
Wamsutter field primarily produces gas. First year gas production for Wamsutter field area is shown on Figure 59. Two areal trends of production can be identified from it. A linear trend on the west shows the largest production from the sand bar associated with Almond Formation. Production from zone B comes from slightly deeper intervals within the Almond.

Figure 59: Location of the seismic survey outlined in red and first-year (gas) production indicated by size of bubbles. 


\section{RefLection Characteristics of Almond Reservoir}

The thickness of the Almond Formation, as derived from well logs through out the survey, varies from 350 to 460 feet. Figure 60 a shows typical well log response for the area. Figure 61 shows the seismic response in the area of the sand bar or barrier island; gamma ray, density and sonic measurements are shown on acoustic impedance (colored) and seismic (wiggle traces) overlay.

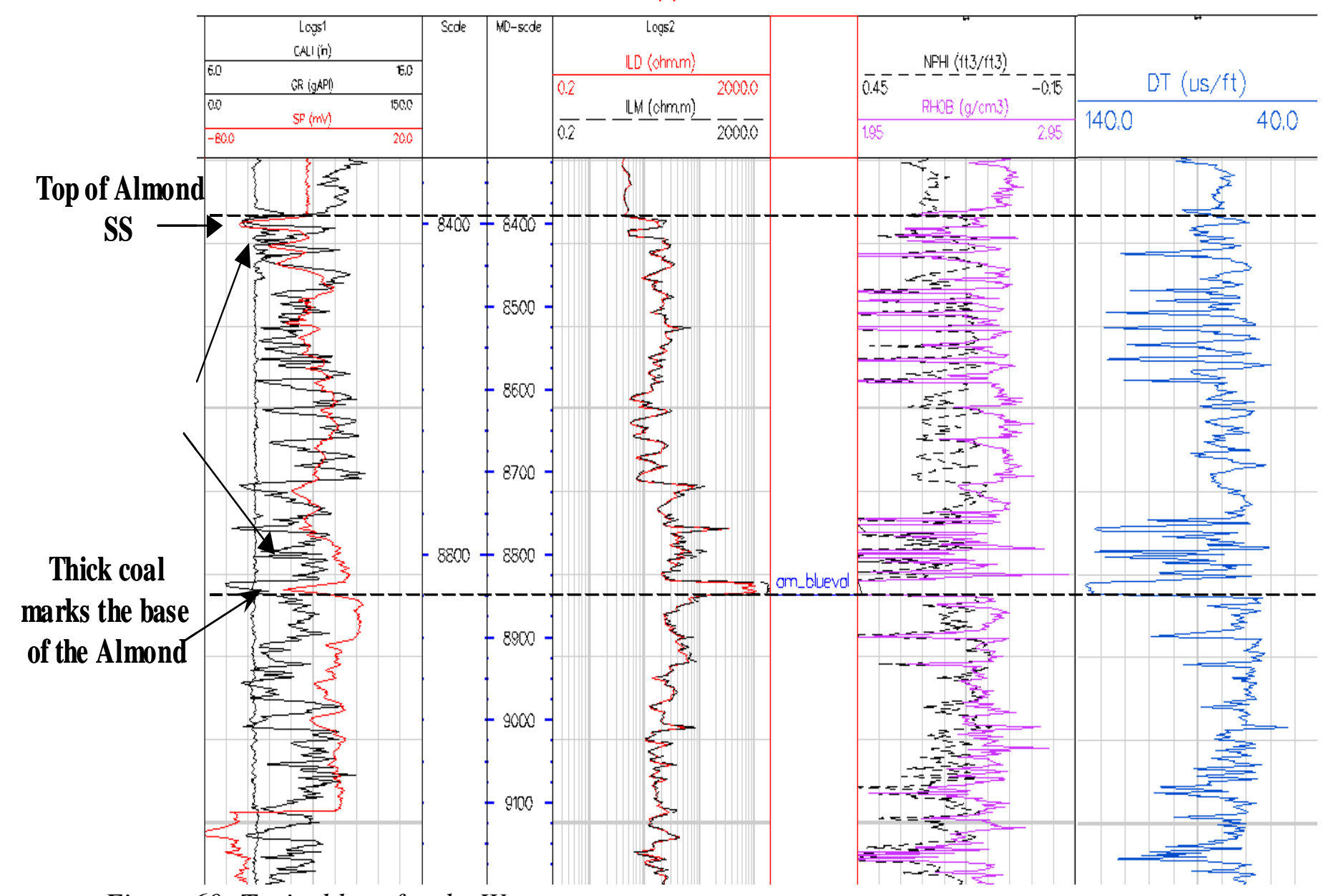

Figure 60: Typical logs for the Wamsutter area.

Although the contact between the Lewis Shale and the Almond Formation is well defined from the gamma ray log and other log measurements, this contact produces mostly a weak negative reflection. Velocity does not change drastically in comparison with the overlaying Lewis shale, and therefore most of the reflection results from the density contrast between these two units. However, within the Almond, interbedded coal layers produce very strong positive reflection. We called this reflection the Almond horizon. Log measurements detect layers of interbedded coals, shales and tight sandstones within the Almond Formation. Typical thickness of these layers varies around 5 feet. At the base of Almond Formation there is a thicker coal bed ( 20 feet thick), which is characterized on the logs by extremely low velocities and densities. This coal bed produces one of the most consistent and well-recognized reflections on the seismic section. 


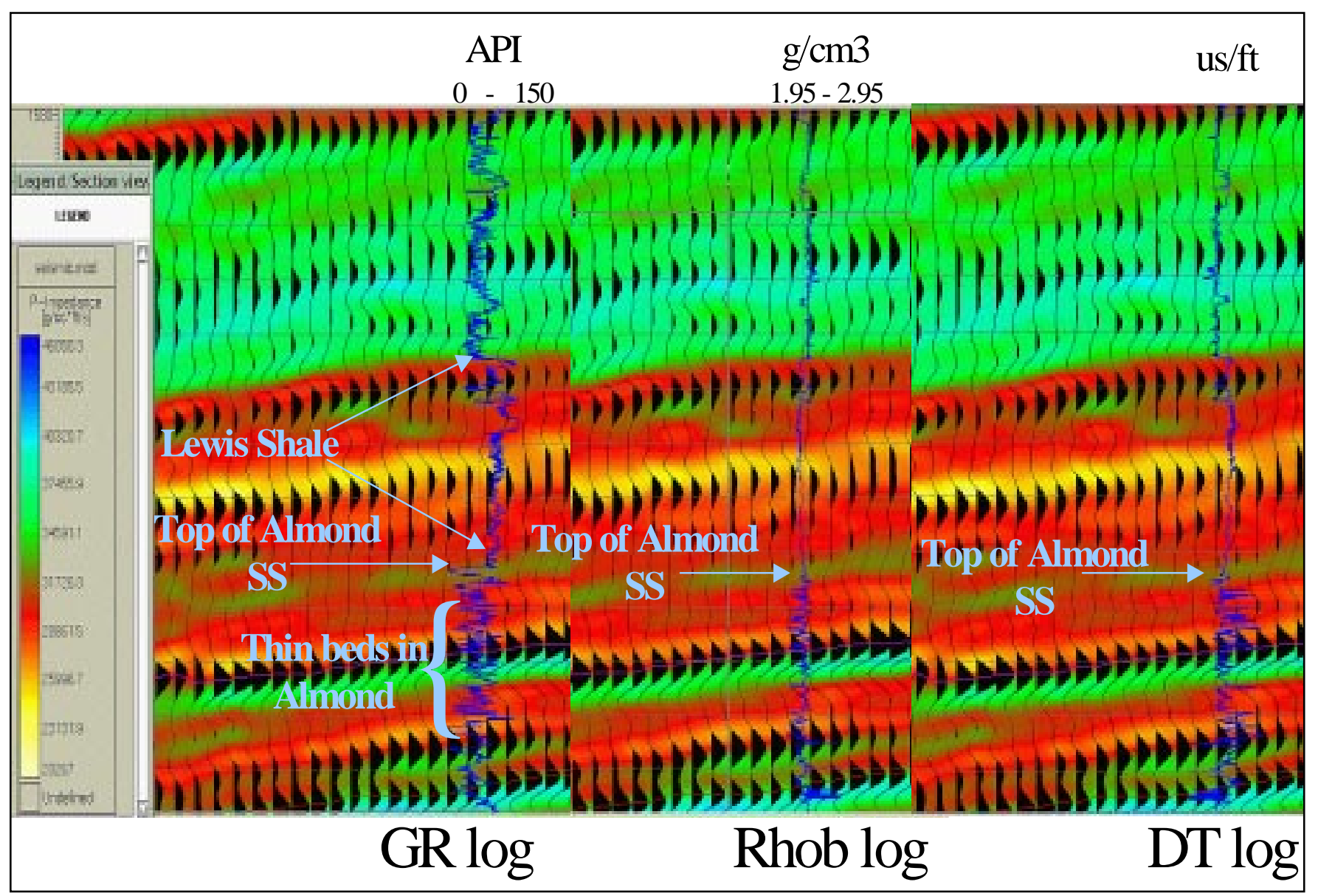

Figure 61: Typical logs of the portion of the Wamsutter field where the sand-bar or barrierisland facies is present.

Seismic data (repeated for each log) are displayed as wiggle lines, and the background colors indicate acoustic impedance resulting from well-constrained inversion.The thin coal beds are apparent from the low density and sonic readings

\section{INTERPRETATION}

Approximately 60-sq. mi. of 3-D seismic data was reinterpreted along several horizons. Those results provide improved structural maps. The use of several high-end software packages made this work easier, providing broader opportunities to evaluate potential attributes in seismic data. Time structure, isochron and attribute maps were constructed.

Figures 62 and 63 show a seismic section through the seismic volume. The Almond seismic horizon is identified and the time structure of the Almond Lower is shown in the map insert. The map shows that horizon is gently dipping to the west. This is a typical time structure for the Wamsutter field as all the identified horizons follow the same pattern. 


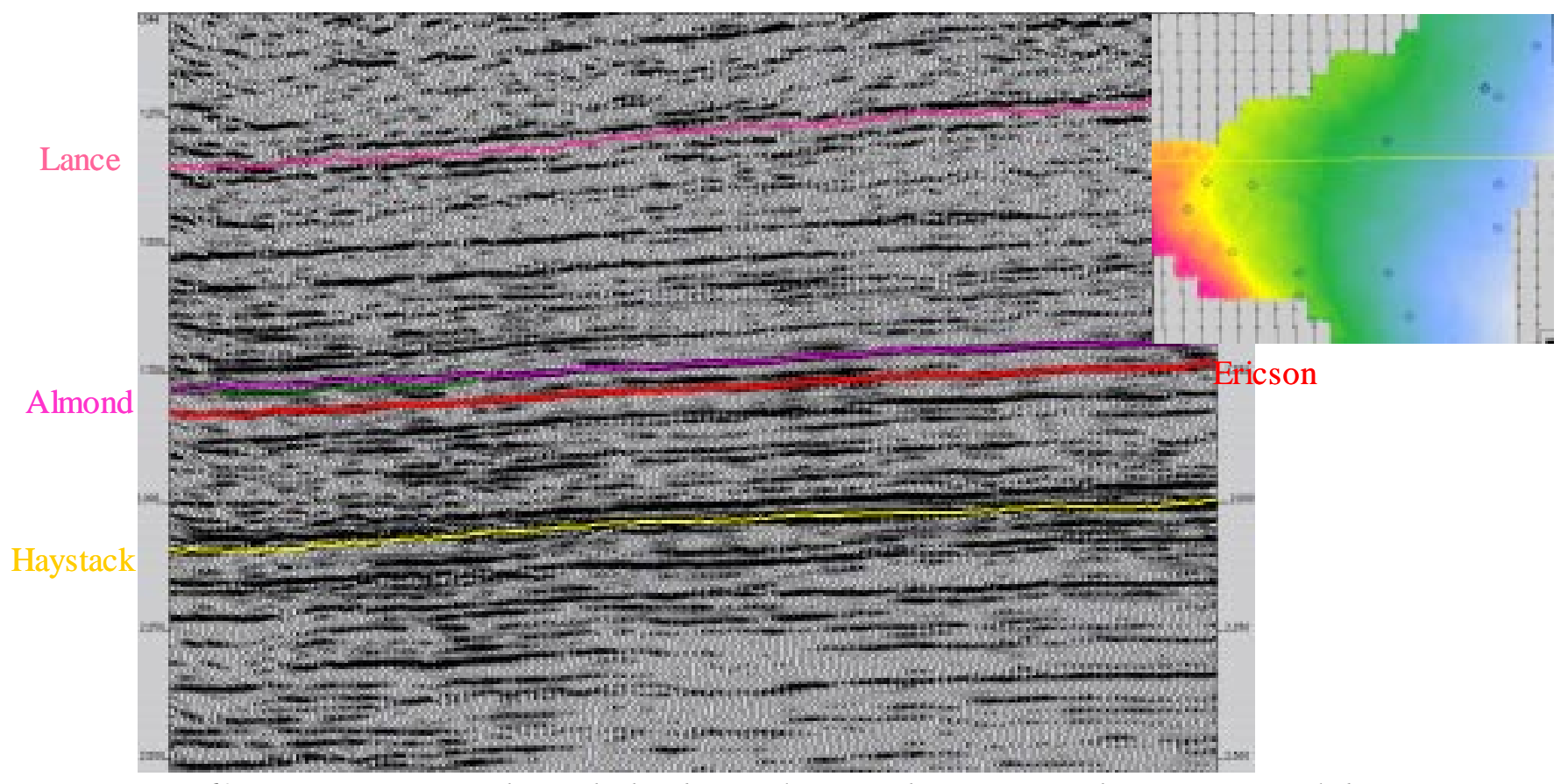

Figure 62: Seismic section through the data volume, with inset map showing general dip

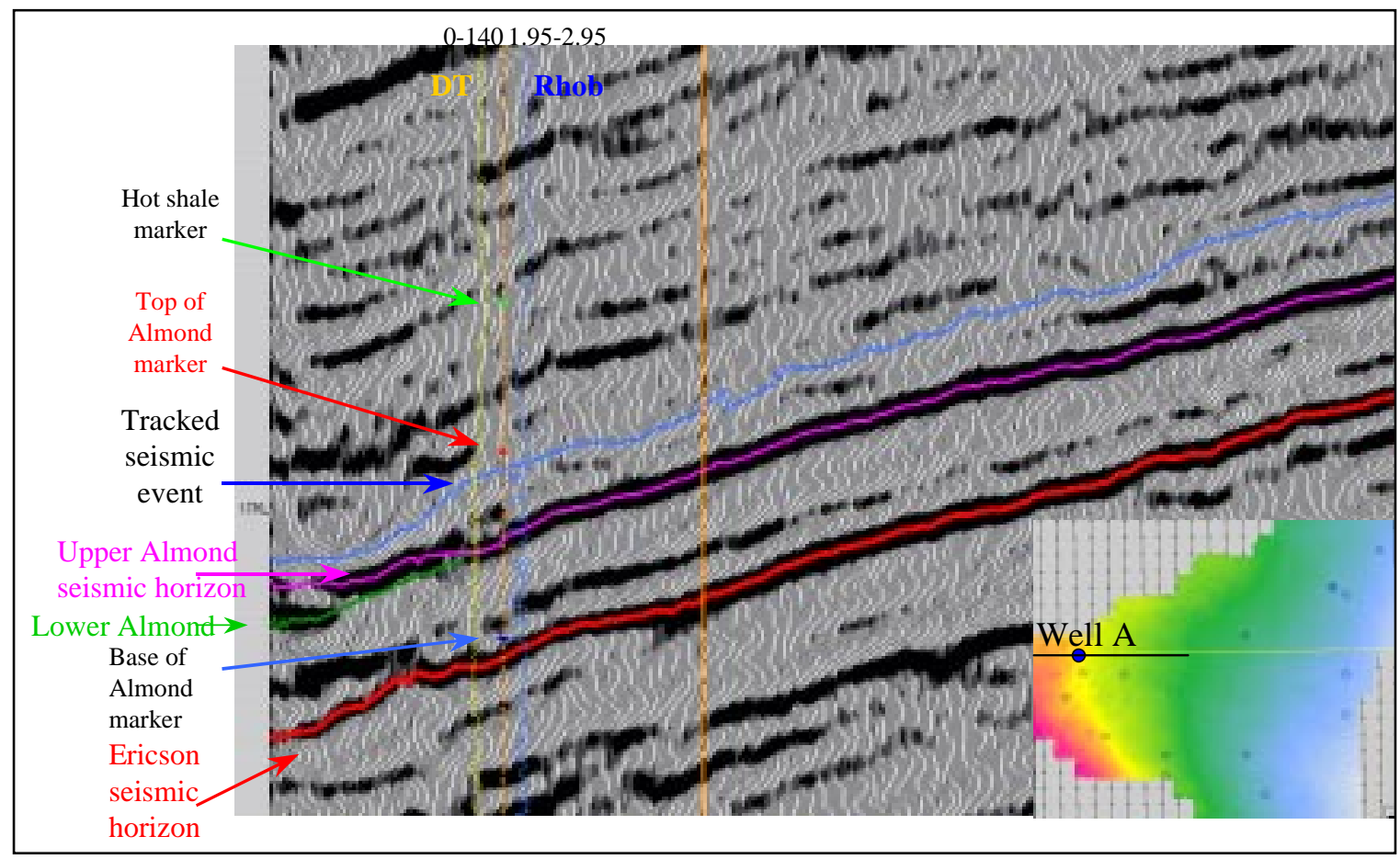

Figure 63: Seismic section in area of the western sand-bar or barrier-island facies.

Note incoherent reflection at location of the sand bar as indicated by 'Top of Almond Marker' from well logs. The nomenclature used here may not be identical to that used in operating company offices. 


\section{Conventional Seismic Attributes}

Various seismic attributes were generated using the Wamsutter data set. Seismic attributes are specific descriptive and quantitative measures of waveform characteristics. Seismic data contains information on time, amplitude, and frequency of the reflected signal. We can extract this information from seismic and use it to characterize seismic data. Attributes can be derived from both pre-stack and post-stack data. They can be calculated along tracked horizon or on the interval.

We first present a series of conventional attribute analyses, which are disappointing in their usefulness, and then (sections 6.2-6.5) present the results of some unconventional attribute analyses, which appear to be much more useful in this study.

\section{Time Derived Horizon AtTRIButes}

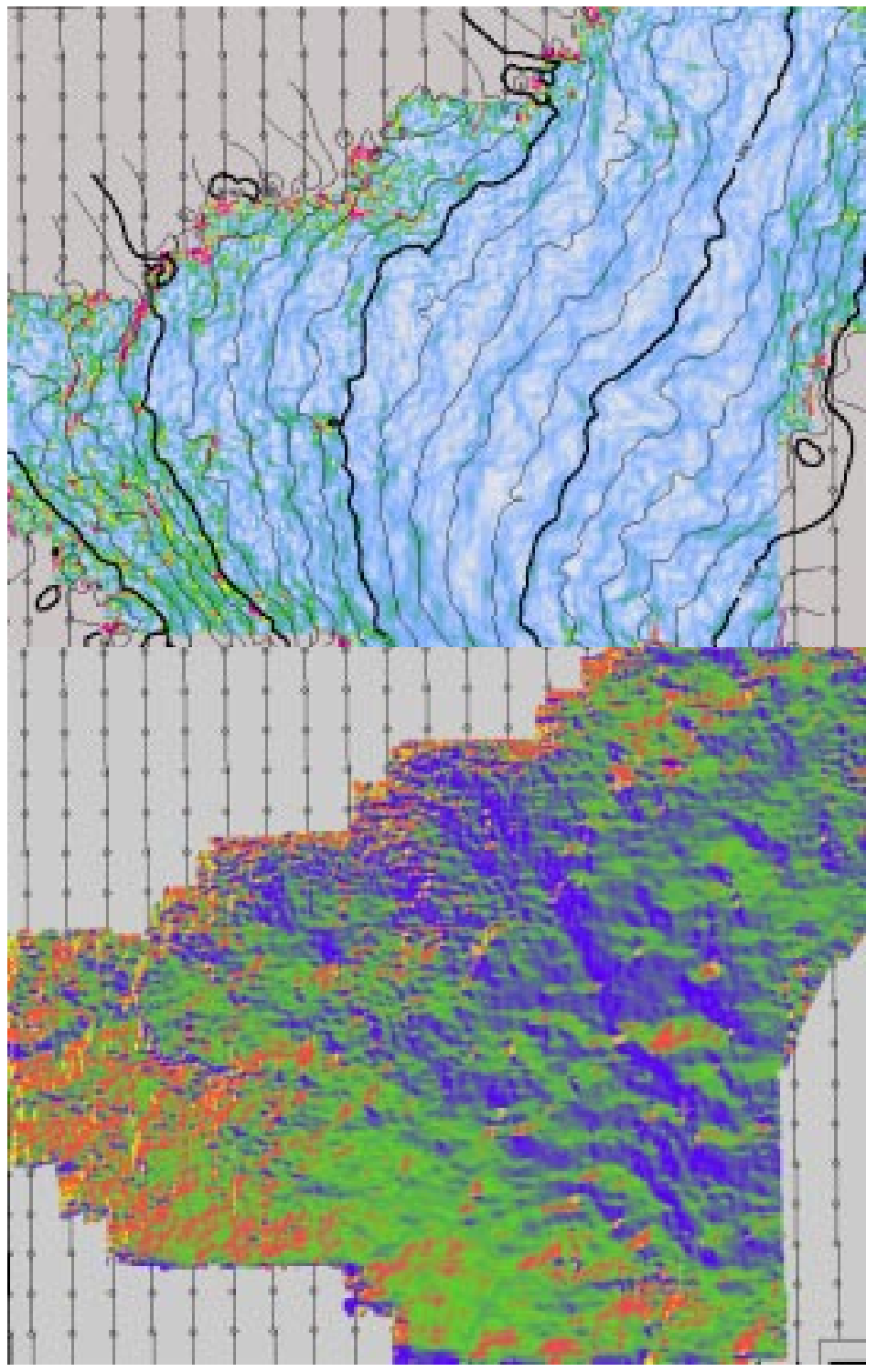

Time-derived horizon attributes were extracted for Almond. Dip, or dip magnitude map is displayed on Figure 64. One time value of a picked horizon is compared with neighboring points and a dip of a local plane is calculated. The dip map shows bad tracking and other artifacts at the edges of a survey. Linear features from the dip map coincide with contours derived from the time structure map.

Figure 64: Dip map with time contours superimposed.

Dip-azimuth maps were also extracted for Almond. Dip-azimuth is similar to the dip attribute but it combines dip and azimuth attributes into one map. Figure 65 shows a dip-azimuth map on Almond-Lower. Dip-azimuth maps are used to improve fault and fracture imaging. Two different color scales emphasize linear trendto: the northeast and the northwest.

Figure 65: Dip-azimuth map with coloring emphasizing two dip directions. 


\section{Window or Trace Based ATtRIBUtes}

A variance cube was computed for the entire seismic 3-D volume to detect possible fracture zones in the Almond. The Gas Research Institute team, during their investigation in 1997-2000, was able to image structural features on some of the horizons in Siberian Ridge Field (approximately 10 miles to the northeast from Wamsutter Field). They identified them as faults and zones of increased fracturing (Sturm, 2000). Coherency, or its inverse variance, measures lateral changes in the seismic response caused by variation in structure by correlating one trace to its neighboring traces and detecting lateral discontinuities. When there is a sudden lateral change of acoustic impedance (lateral reflectivity may vary because of changes in lithology,

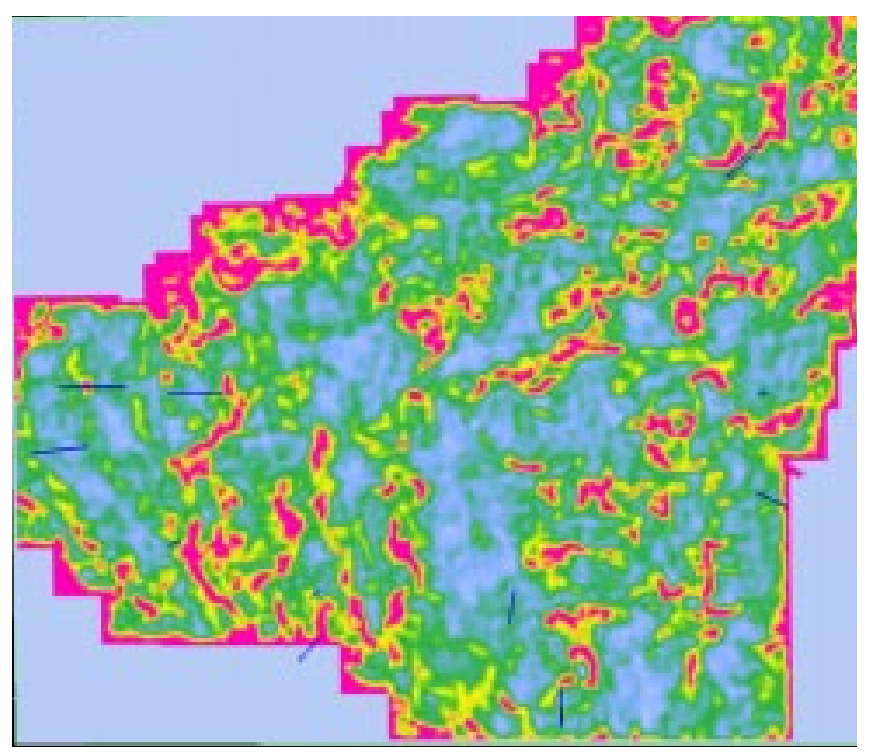
fluid, and thickness) coherency may be able to detect these changes. Coherency is commonly used to detect faults, help, guide, and perform the quality control of the interpretation. It is also independent of tracking involved in horizon interpretation because it is being calculated over the interval or on the whole seismic trace. Figure 66 shows a time slice trough variance cube corresponding to times of the Almond Formation. Curvy and linear features are apparent, and sand bar location are unidentifiable.

Figure 66: Coherence time-slice at $1862 \mathrm{~ms}$ Red represents incoherent features.

\section{AMPLITUde ATTRIBUTES}

Several conventional amplitude attributes were extracted in Wamsutter for different horizons. In general, in conventional interpretations, amplitude attributes point to areas that may contain hydrocarbon accumulations. We describe some commonly used attributes below. We extracted them at some interval (usually from Lower Almond seismic horizon and up to some time interval above) in order to include the top of the Almond formation (which does not generate a strong reflection and therefore is not tracked on seismic data but might contain a significant hydrocarbon potential).

RMS amplitude map on the interval from Almond Lower up to 70msec above is shown in Figure 67. RMS amplitude is a measure of the reflectivity within a time window. To be more specific, it is the square root of the sum of the squares of the amplitudes within the window interval. It is conventionally used as a direct hydrocarbon indicator in a zone. RMS amplitude attribute might be more useful than absolute reflection amplitude because it can be obtained over an interval, while the amplitude alone is extracted along the horizon. Horizon tracking can influence the value of amplitude extracted along the horizon. 
Sum of magnitudes amplitude attribute (SMAA), which might serve as a lithology and porosity indicator was extracted for the same interval. It is the arithmetic mean of amplitudes multiplied by the number of samples in the window and is independent of amplitude sign. This attribute provides a measure of brightness volume, because SMAA is just the arithmetic mean of amplitudes multiplied by formation thickness in time (Figure 67). Sum of magnitudes and RMS attributes look very similar in the map view. Those two attributes indicate anomalous amplitude zones which are very similar in shape.

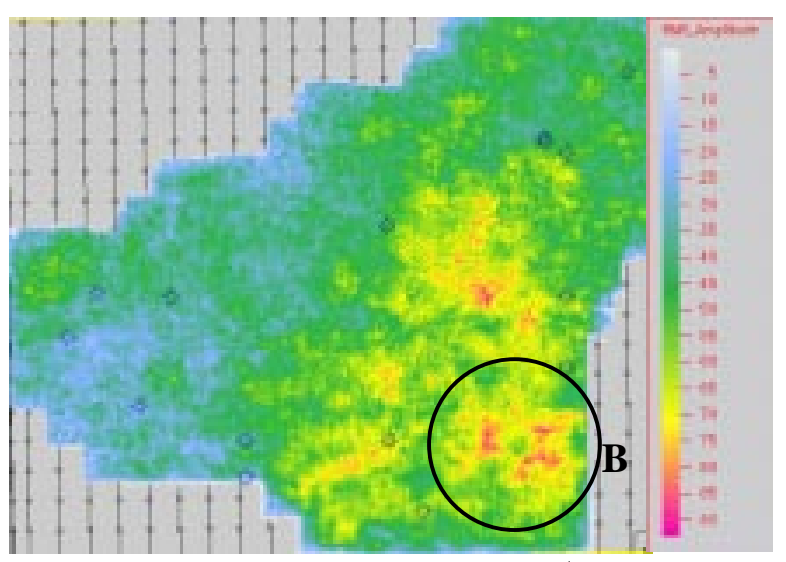

RMS attribute

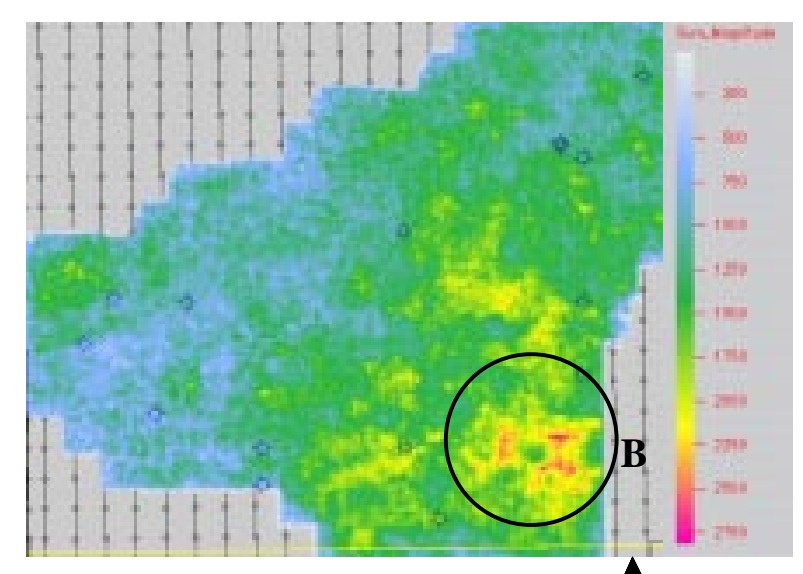

Sum of magnitudes attribute

Figure 67: Maps of two amplitude-based attributes.

\section{FREQUENCY ATTRIBUTES}

Energy half-time attribute is the proportion of time required for the energy within the window to reach one half of the total energy within the entire window. This attribute is often used to identify sand-shale lithologies. If an interval has more sands at the top, the unit might be characterized by a strong reflector, front loading much of the energy towards the upper part of the interval. A shale-rich interval may have a weaker reflection at the top. Thus, the energy halftime attribute may show shorter times for the sands. Energy half-time attribute was derived on the interval Almond Lower and 70msec above. Figure 67 compares attributes described earlier extracted on the same interval.

Instantaneous attributes can be calculated from time, energy, frequency and attenuation, derived from the complex trace analysis (Taner et al 1977, and Taner et al 1979) of seismic trace data at the event location. Various instantaneous attributes were calculated, but are not displayed here. In general, they did not provide insight into the reservoir properties.

\section{SEISMIC INVERSION FOR ACOUSTIC IMPEDANCE}

Seismic inversion is another window or trace based attribute in which each trace is inverted for acoustic impedance. We inverted the entire 3-D seismic volume. Inversion is a powerful tool, making it easier to interpret horizons, faults, stratigraphic units, and recognize geology. Inversion reveals interpretation errors; removes wavelet tuning effects and reduces side-lobes; extends beyond the seismic bandwidth; and allows us to make quantitative predictions of reservoir properties. 
Acoustic Impedance (AI) is product of density and P-wave velocity. AI is a rock volumetric property and not an interface property like seismic data. AI inversion is simply the transformation of seismic data into pseudoacoustic logs at every trace making use of the seismic data, guided by the log data in the volume.

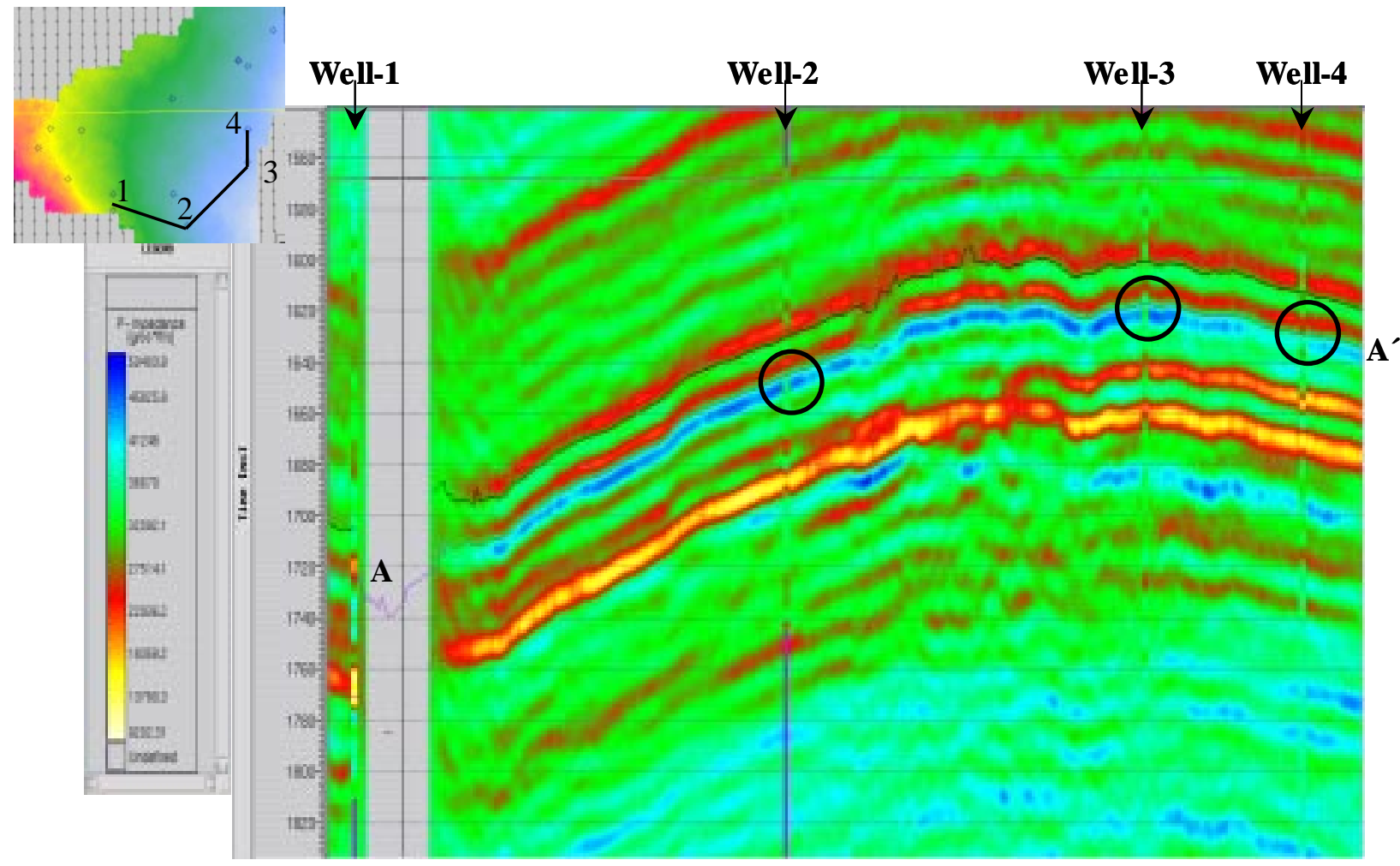

Figure 68:Profile through a well-section of the inverted volume.

The circles identify zones where the acoustic imedance determined by the inversion is significantly higher than that obained from the well logs.

Figure 68 shows the final inversion result. Some unusual observations may be made from this. In some areas through the inverted volume AI values are higher than AI derived from the well data. For instance, one of those zones is marked A- $\mathrm{A}^{\prime}$ and is located below the tracked Almond horizon. This observed phenomenon may be caused by hydrocarbons in the interval, through a hydrocarbon production related effect, or through amplitude-versus-offset effects. If the logging was done prior to the seismic acquisition, then high AI values not present in the well log data might be caused by some changes related to production. As an effect of production the rock matrix stiffens as the fluid pressure decreases. This increase in confining pressure leads to an increase of impedances around well locations. The increased impedances are not near the well locations, leading to the conclusion that production has not significantly affected velocities in this field.

Amplitude variation with offset (AVO) effect can introduce brightening or dimming of stacked seismic amplitudes. Acoustic impedance derived from well data is equal to AI from the normal incidence case. But the amplitudes in the seismic data come from far and near offsets. An increase in amplitude with offset could result in a larger amplitude on a stacked data, which we then mis-interpret as zero-offset amplitudes. One way to test if this is the case would be to 
model the AVO behavior from well data; this requires knowledge of the shear-wave velocities in the well, which we do not have.

\section{SWEET SPOT INVESTIGATION}

Often exploration in basins like the Rocky Mountains basins is not economically successful. Gas is present in the target horizon but flow rates are unsatisfactory low. There are models that suggest that in order to obtain a good production of basin-centered gas, investigators look for "Sweet Spots" or areas of anomalous high hydrocarbon production. According to the USGS definition of Sweet Spot, it is an area of relatively higher productivity within a continuous hydrocarbon accumulation (Figure 69). The causes of Sweet Spot occurrence might vary and different researchers have developed different approaches to the Sweet Spot concept. Yin and Surdam (1993) correlate Sweet Spots with higher than average porosity. Surdam concludes that some Sweet Spots are caused by local overpressure. Iverson (1993) focuses on permeability and flow rates. Billingsley (1999) defines Sweet Spot strictly according to economic standards without reference to cause.

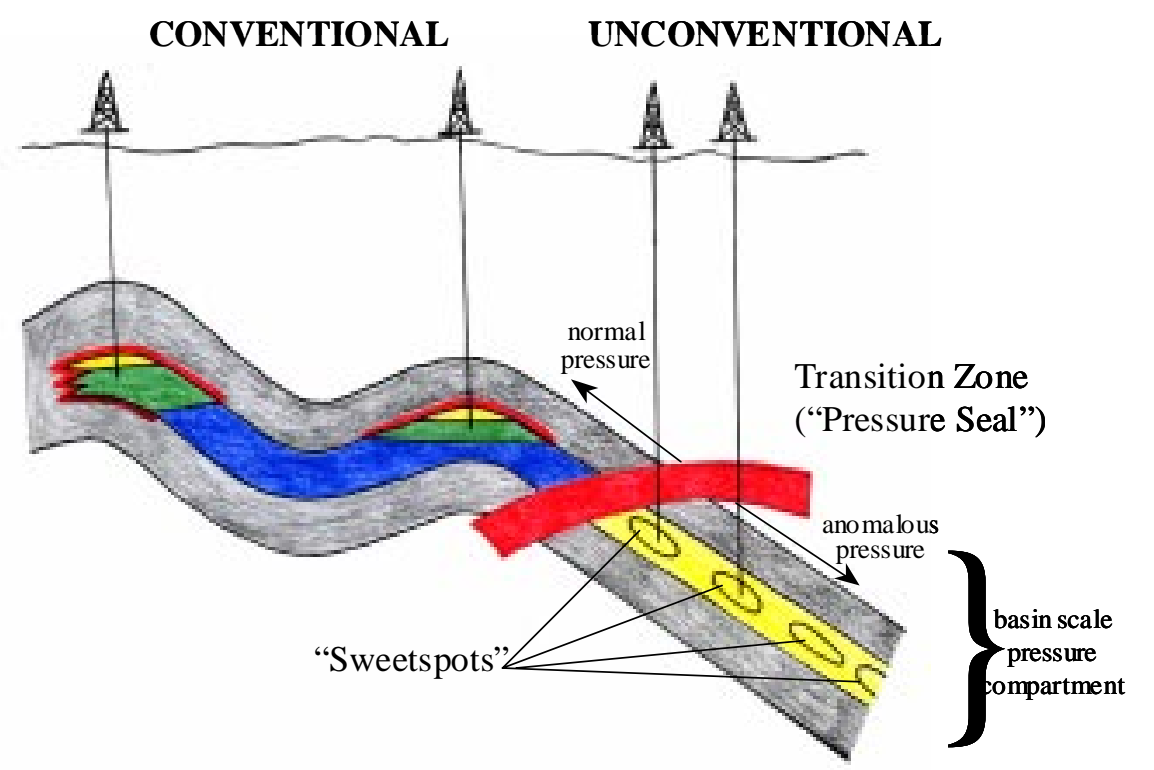

Figure 69: "Sweet Spot" scenario, from (Parter, 2001)

Having generated a number of attributes, we attempted to identify sweet spots in Wamsutter data. Parker et al (2001) has suggested that in Greater Green River Basin sediments are overpressured. In overpressured areas a decrease in velocity usually indicates decrease in effective pressure, and therefore an increase in pore pressure, i.e.,

\section{Effective pressure $=$ Confining Pressure - Pore Pressure.}

The effective pressure anomaly within the interval will be caused by increase in pore pressure. A pore pressure increase is strongly suggestive of a fluid trap.

Researchers from University of Wyoming conducted a similar study in the Siberian Ridge Field, approximately 10 miles northeast of Wamsutter field. They reprocessed a volume of pre-stack seismic data and determined the volume of abnormal velocities from stacking velocities in the Lewis Shale. 
We did not have pre-stack information from which we can determine abnormal stacking velocities. But we have computed a volume of acoustic impedance. We can determine the areas of general velocity decrease by monitoring behavior of $\mathrm{AI}(\mathrm{AI}=\rho \mathrm{V})$ and searching for low values of AI along each stratigraphic horizon. We performed this on an interval starting $100 \mathrm{msec}$ above Almond Upper seismic horizon and extending upward into Lewis shale and above. The total vertical thickness of the interval is approximately $380 \mathrm{msec}$ in two-way travel time. In some similar basins, the shales contain hydrocarbon potential. Some production occurs within Lewis shale and above it. Our study interval was bounded by two roughly (but not precisely) parallel horizons (Figure 70).

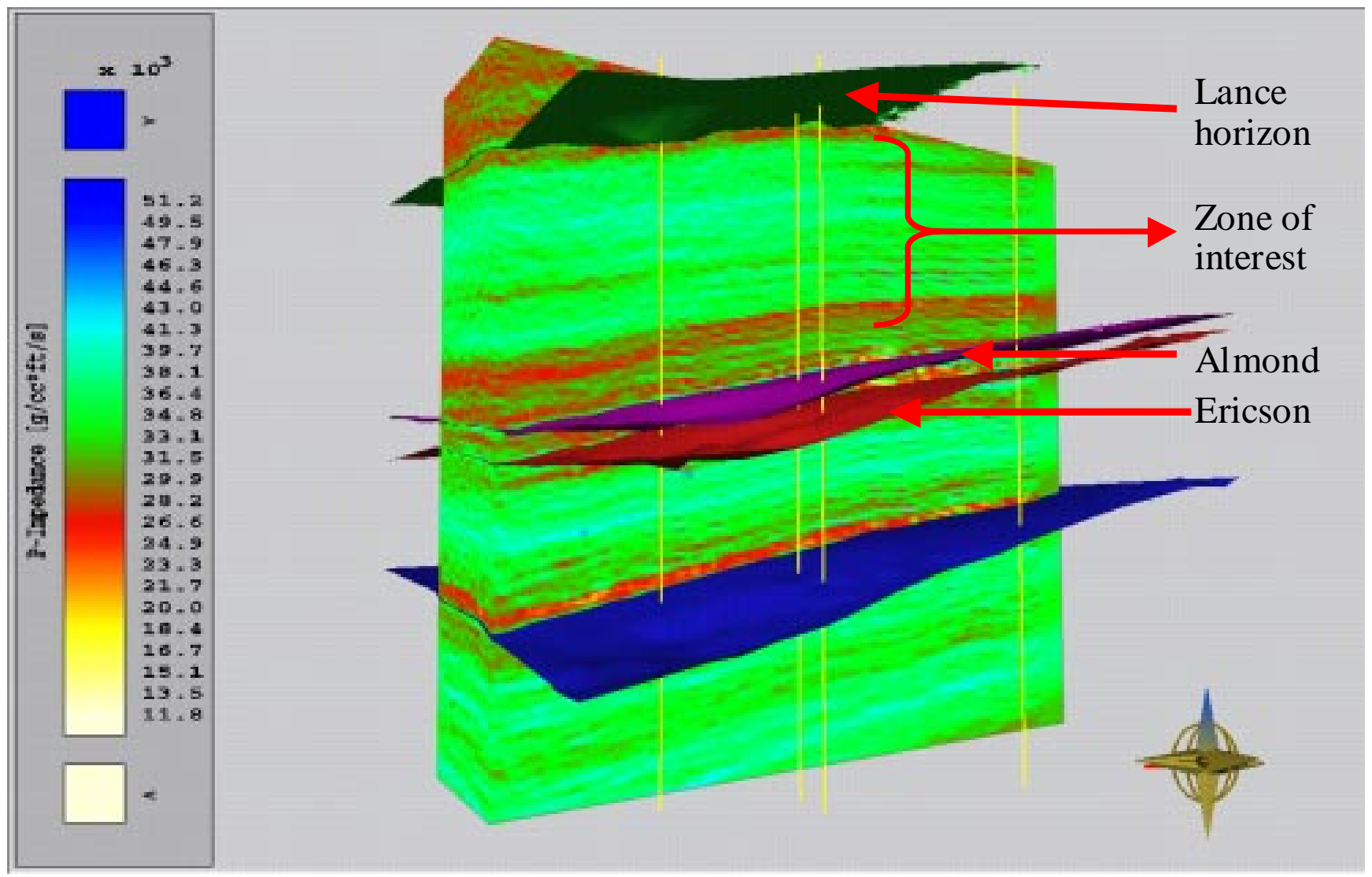

Figure 70: A portion of the inverted volume with specific horizons indicated.

In order to obtain abnormal AI values we divided the interval into smaller slices; those slices (each about 20msec thick) follow the shape of tracked seismic horizons. Because the layering is close to comformable, we were able to track beds within these volume slices (Figure 70). For each of the slices, the average AI value was removed, indicating abnormal AI values. We identified the abnormally low AI values, and found that together, they formed "clouds" or bodylike shapes. Those "clouds" were checked for connectivity and grouped into bodies according to size. This approach enabled us to obtain a 3-D model of possible abnormal pressure distributions within the Lance-Lewis and Upper Almond interval (Figure 71). 


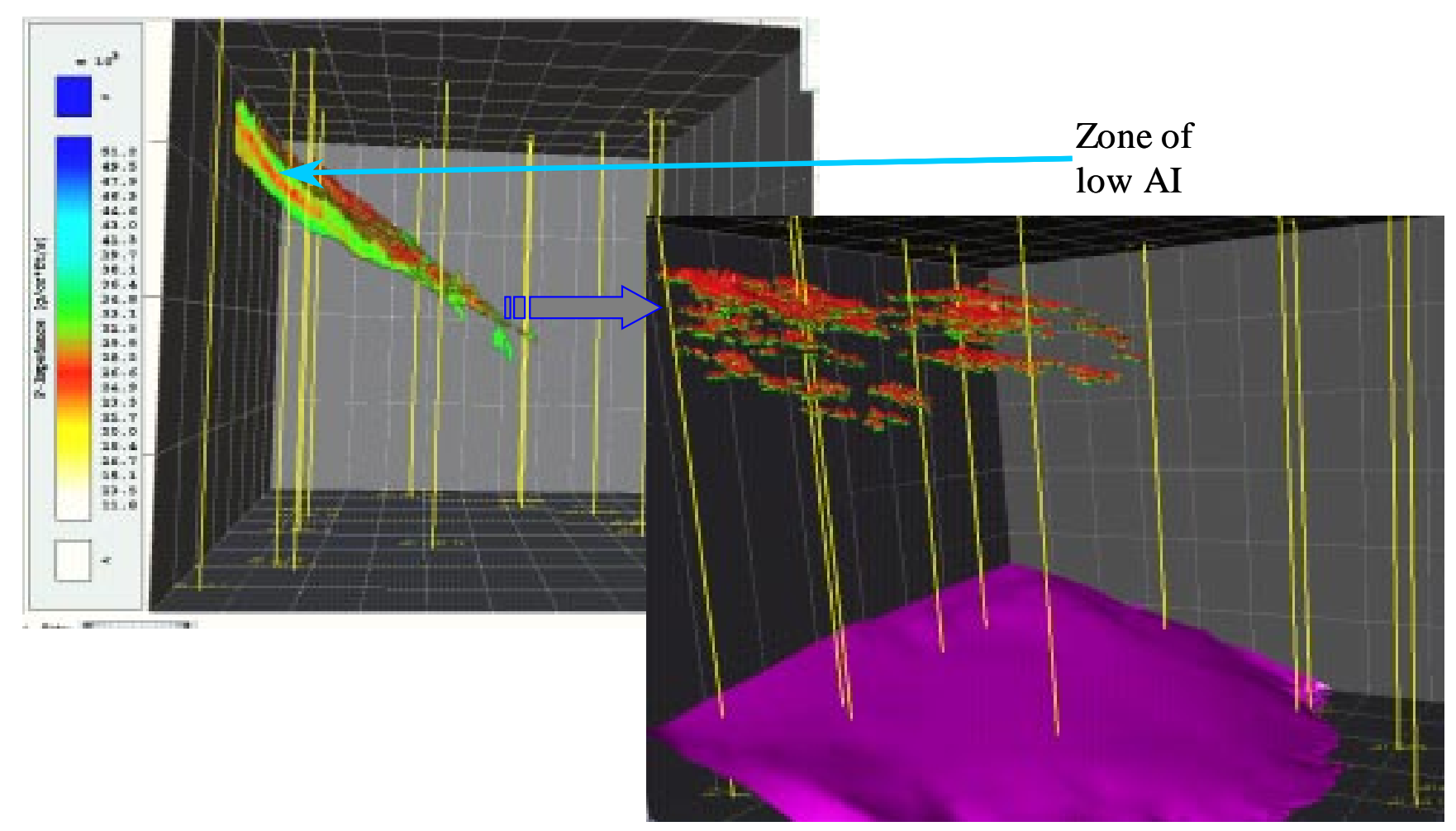

Figure 71: Extraction of low-impedance bodies within small intervals.

We do not have pressure information in the Lewis shale in this portion of the basin, and it does not appear to contain hydrocarbons, but the method used here may be useful in actual prospecting environments.

\section{COHERENCY ATTRIBUTE ANALYSIS}

The largest gas production comes from the sand bar deposit in the western part of the study area. We decided to look at the very top of Almond Formation, which corresponds to the sand bar, and try to identify it on the seismic data. As was mentioned before, the top of the Almond Formation is well recognizable from gamma ray log, but the top produces a weak and inconsistent seismic reflection. This reflection is especially variable in the area of the sand bar (Figure 72a), where the downlap of features associated with the growth of the sand bar is apparent. We decided to try to 'quantify' this changing character of the wavelet. Coherency, or its inverse variance, is the attribute that can measure the lateral discontinuity in the change in acoustic impedance. Time slices through the coherency volume did not provide useful information (Figure 66); probably due to the dipping beds. A horizon-based approach is more appropriate. Maximum variance was extracted as a new attribute from several time intervals above the tracked Almond horizon in order to image the top of the Almond. Figure $72 \mathrm{~b}$ shows several maps of maximum variance for successive intervals as we move upward from tracked Almond horizon. After comparing these maps for different intervals we concluded that the best production trend can be correlated to the maximum variance, particularly with the sand bar interval at 32-38msec above the Almond Lower horizon (Figure 73). There is a good correlation between best production and the incoherent events as indicated by the variance attribute. We think that those incoherent features correspond to the sand bar and represent ancient shorelines, or paleobeaches, and near offshore facies. 


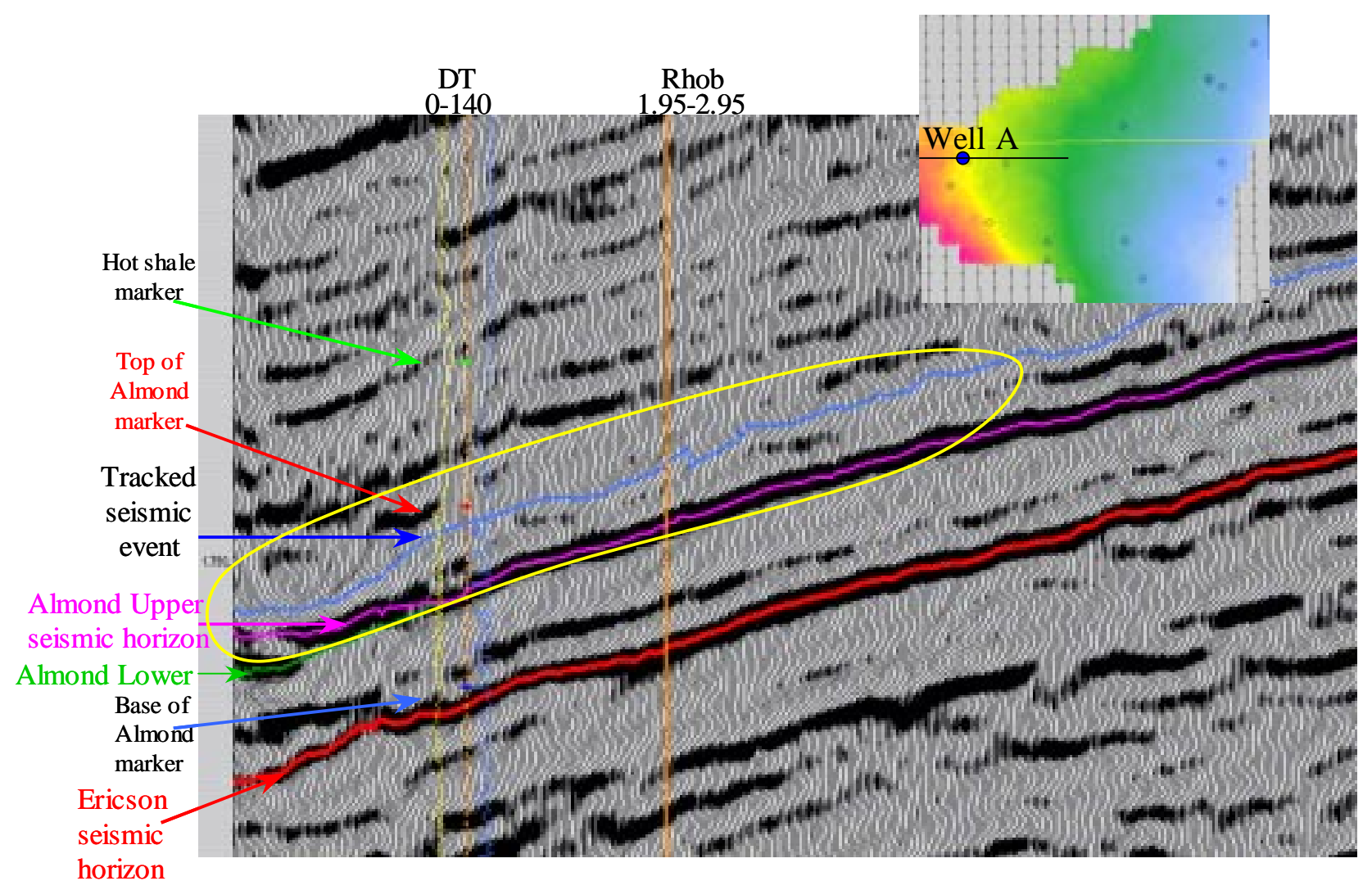
horizon

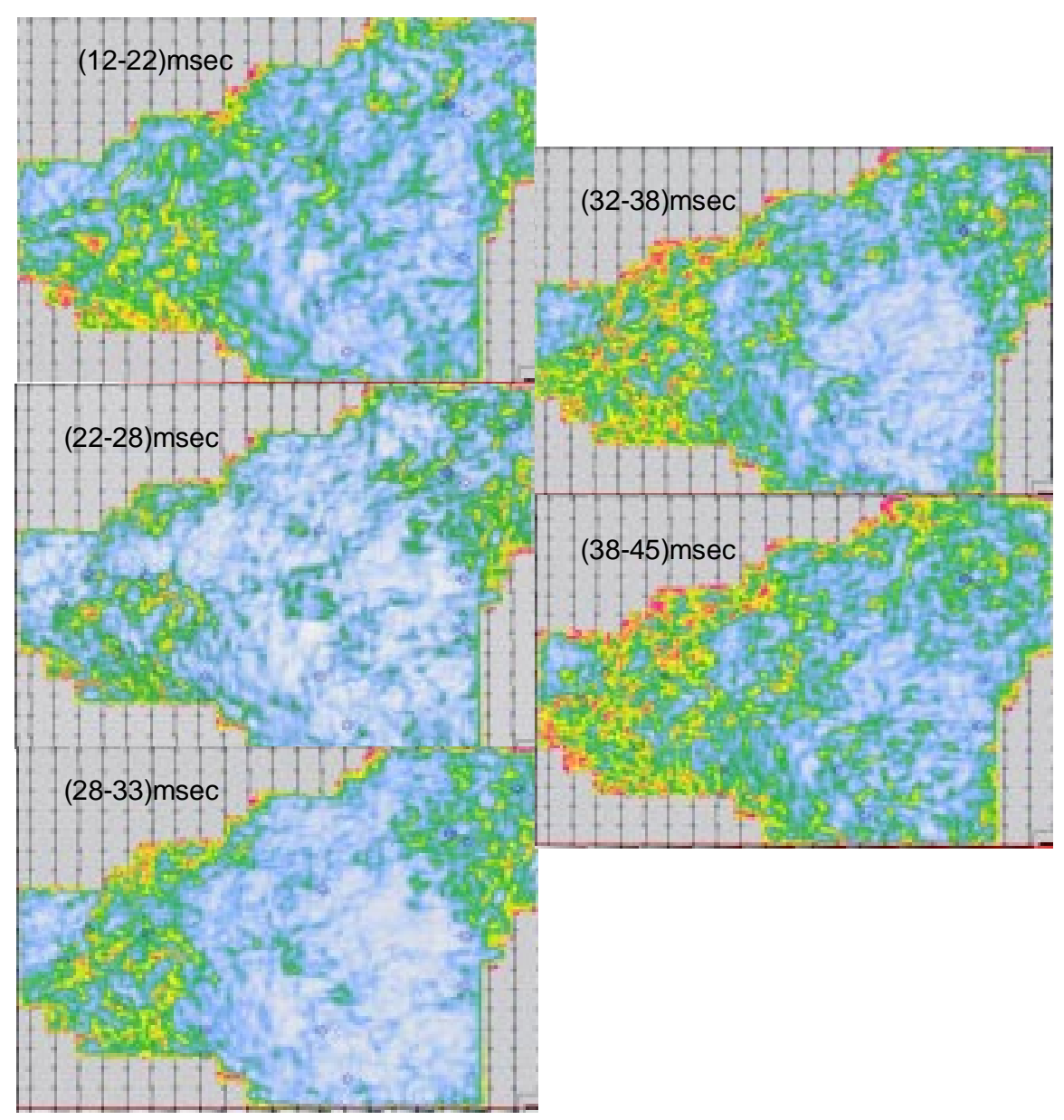

Figure 72a: Seismic section in area of the barrier island or sand bar; its location is circled.

Figure 72b: Variance (incoherency) attributes over several horizon-based windows as labeled in $\mathrm{ms}$ above the Almond.. 


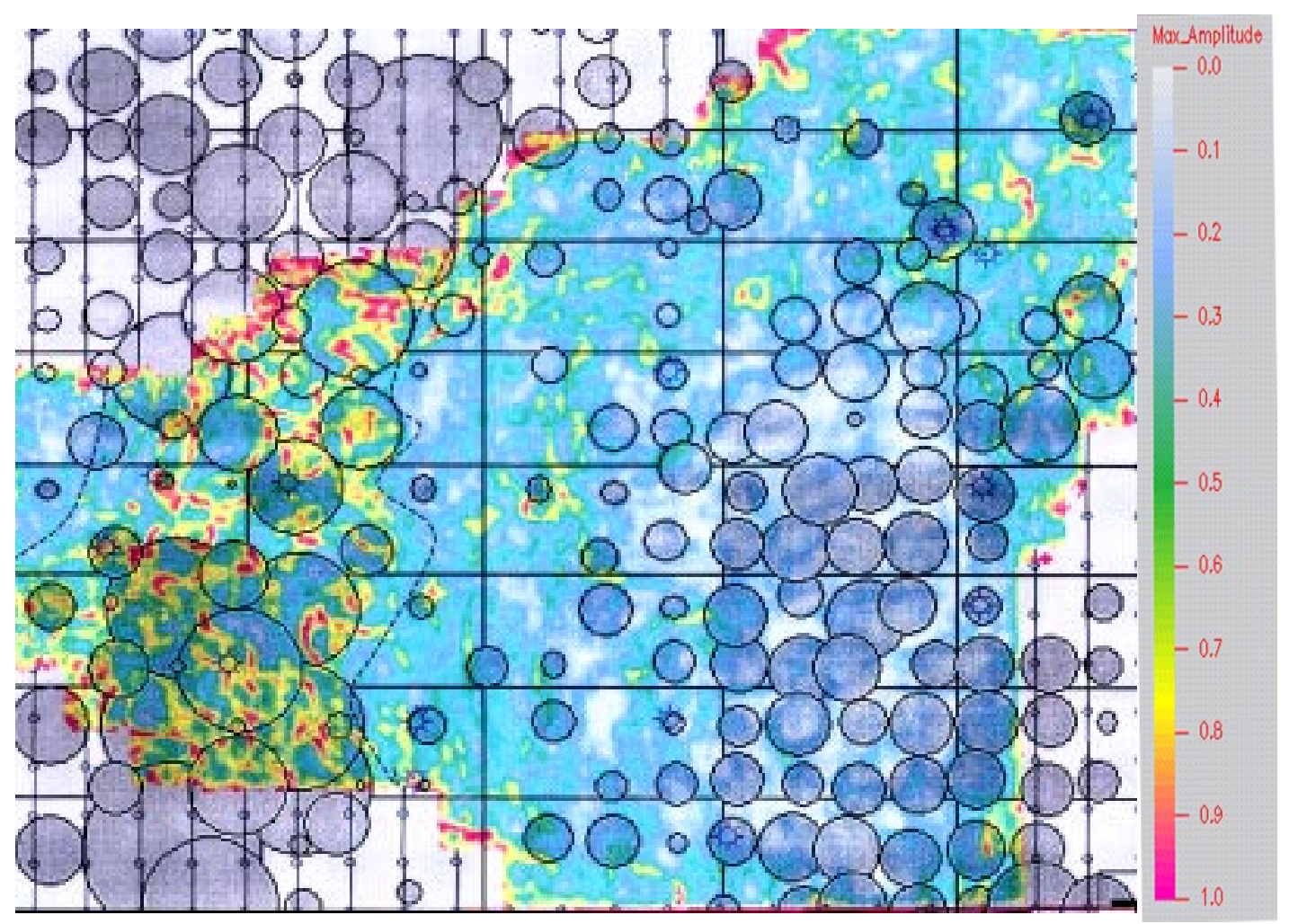

Figure 73: Maximum variance in

Perhaps there is some correlation between other attributes and first year gas production but this is not very obvious. For example, energy half-time (Figure 74, left image) shows no apparent correlaation with production, but sum-of-magnitudes amplitude (right image) correlates with production in the southeast, where production is from the lower Almond. The summing was taken over a 70-ms interval from the lower Almond upward.

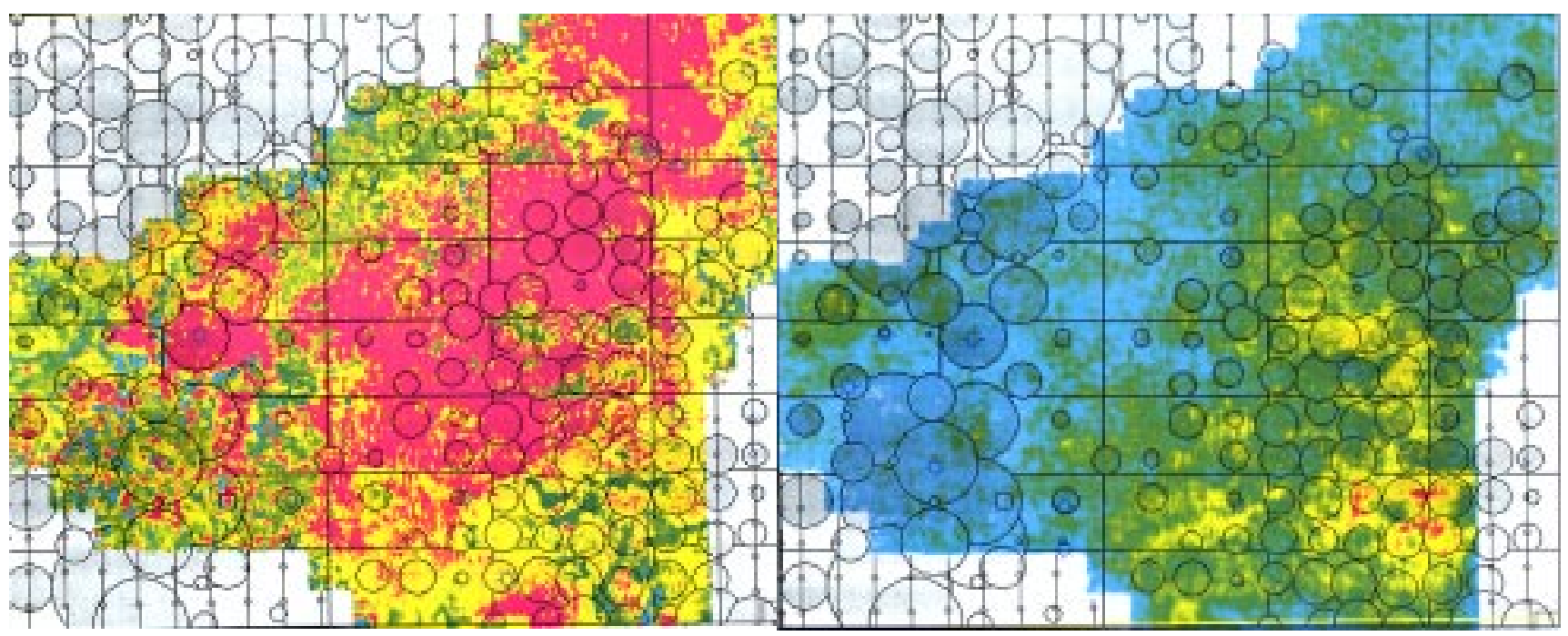

Figure 74: Energy half-time (left) and sum-of-magnitudes amplitude (right) shown with firstyear production. 


\section{Dynamic Fluid Method at Wamsutter:}

The Wamsutter data set was processed using Dynamic Fluid Model (DFM) technique by Transseismic International Inc, who provided the results of their processing to Michigan Tech. Their complete report is provided in the appendix to this report

The attribute computed is considered by Trans Seismic to be instantaneous estimates of the anomalous pressure gradient $\theta_{i}$ (Figure 75), and maps of anomalous pressure estimates can be created for seismic horizons.

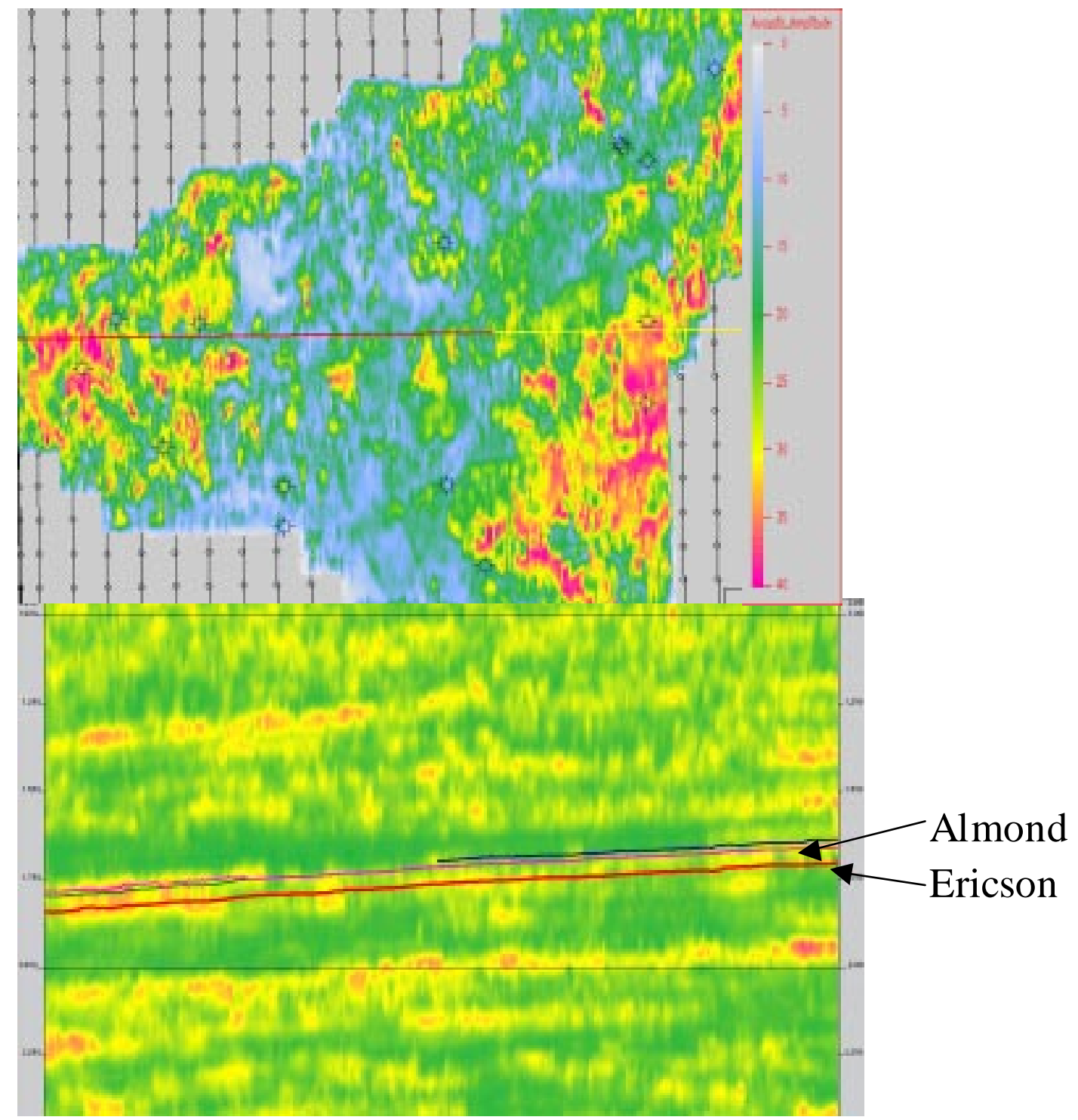

Figure 75: DFM map and section showing anomalies

The map is on the lower Almond; the color bar indicates values of the pressure attribute, with red being maximum fluid pressure or minimum effective confining pressure.

We (Michigan Tech) compared the DFM results in the Lewis shale with our results for lowimpedance intervals (that is, those zones that are lower-than-typical within various small intervals) discussed in an earlier section of this report, since both attributes were designed to identify zones of anomalous pressure and, perhaps, microfracturing. 
Figure 76 shows another vertical section through the DFM volume, focussing on the area of interest. Only the highest DFM amplitudes are visible on Figure 77. These highest continuous amplitudes were captured, grouped and saved as bodies (Figure 78).

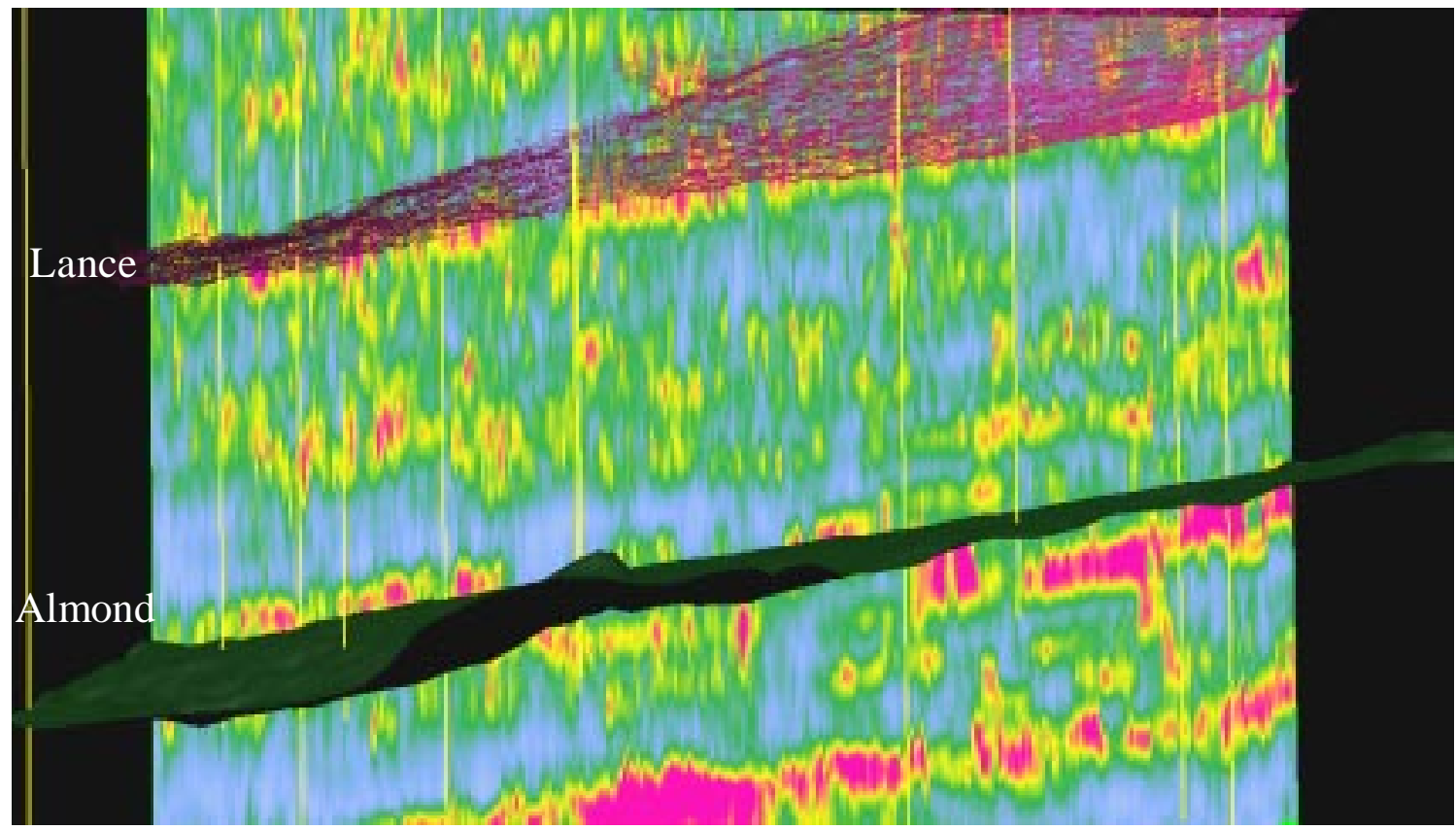

Figure 76: DFM section showing the Lewis shale region between Lance and Almond formations.

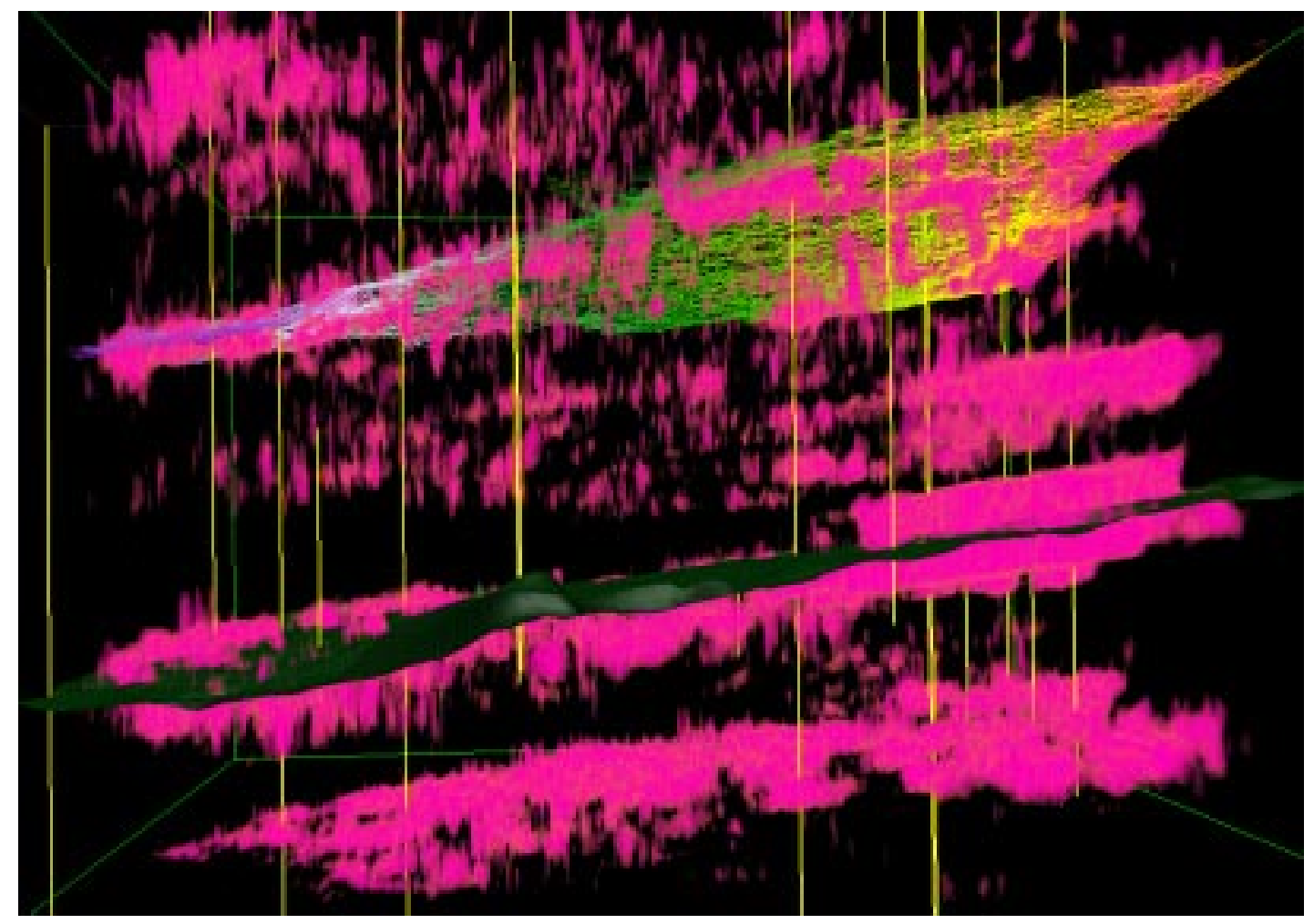

Figure 77: $3 D$ view of the most extreme values of pressure attribute from DFM. 


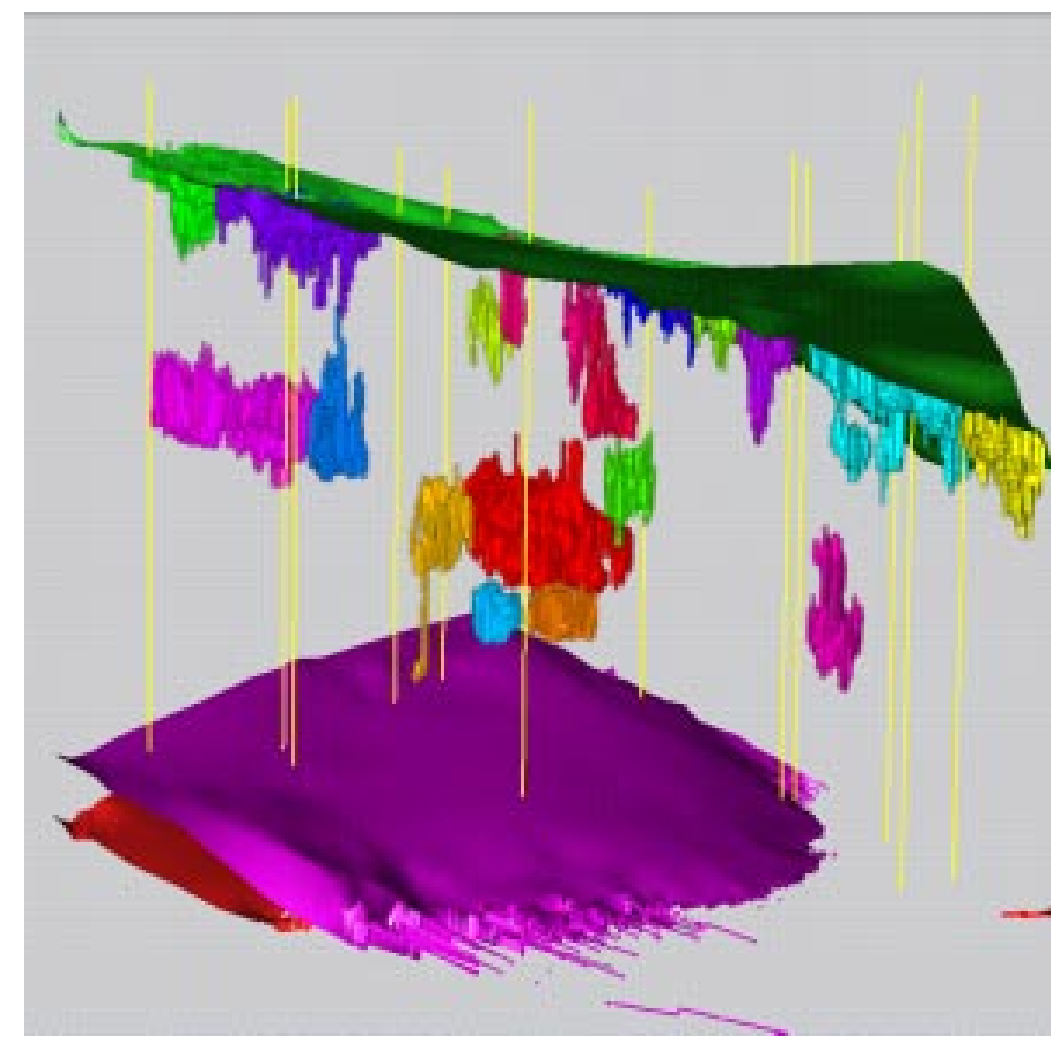

Figure 78: Collected bodies of anomalous pressure values from DFM analysis.

Figure 79 shows a map view of DFM bodies. We compared the time thickness of the bodies and derived an RMS DFM amplitude. Apparently the highest RMS DFM values correspond to the areas of high fluid pressures in the DFM model.

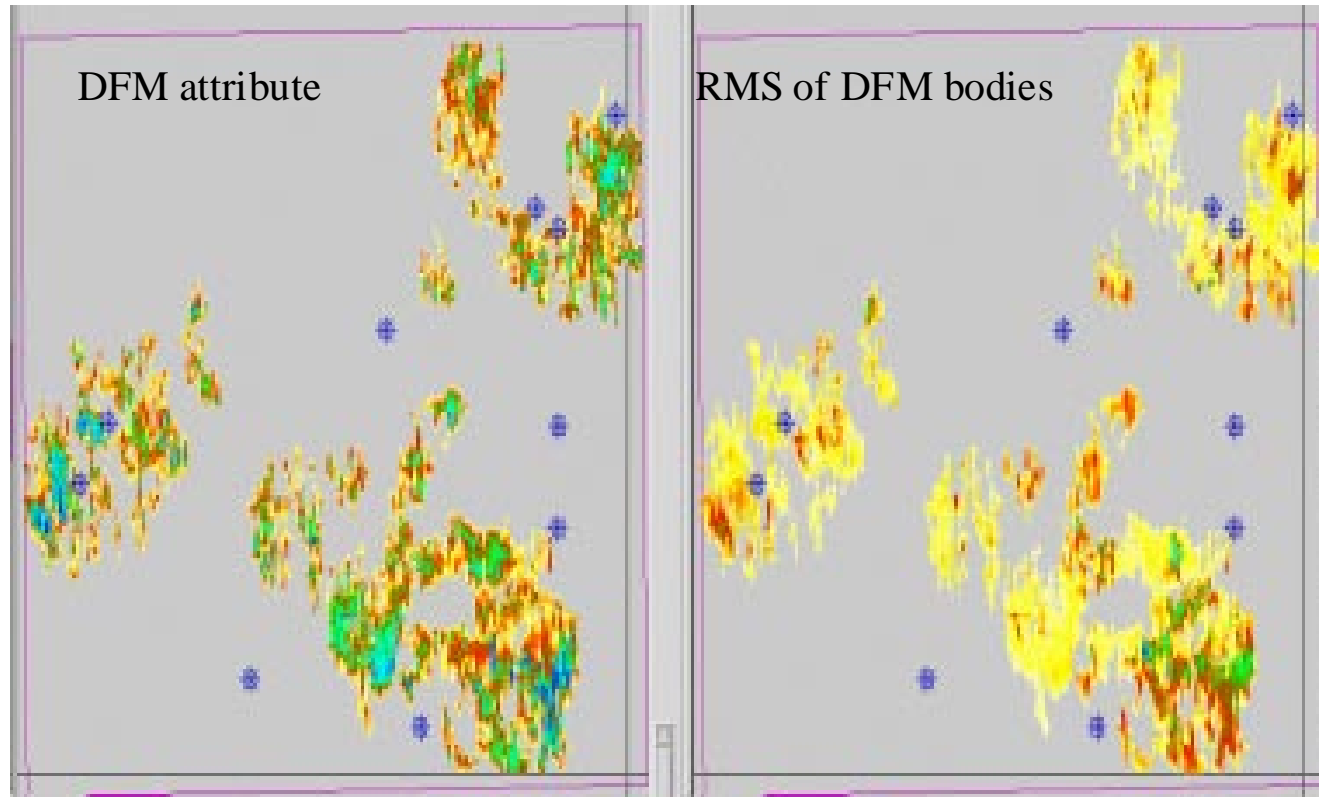

Figure 79: Summed values of the DFM anomalies and the RMS amplitudes of these summed bodies over the eastern portion of the survey area.

As described earlier, we also derived anomalously low acoustic impedance (AI) values in the Lewis formation, from $100 \mathrm{msec}$ above the Almond horizon to the Lance horizon. Maps of low AI bodies and DFM derived high fluid pressures can be compared (Figure 80). Both of these attributes should indicate areas of high fluid pressures. Note the coinciding areas on the south 
and northeast on both maps. Through comparison of the results from the DFM analysis, the anomalously low acoustic impedance summing, and the RMS amplitudes suggests that these attributes indicate pressure compartmentalization in the Lewis shale, but there is insufficient $\log$ data to test this model.

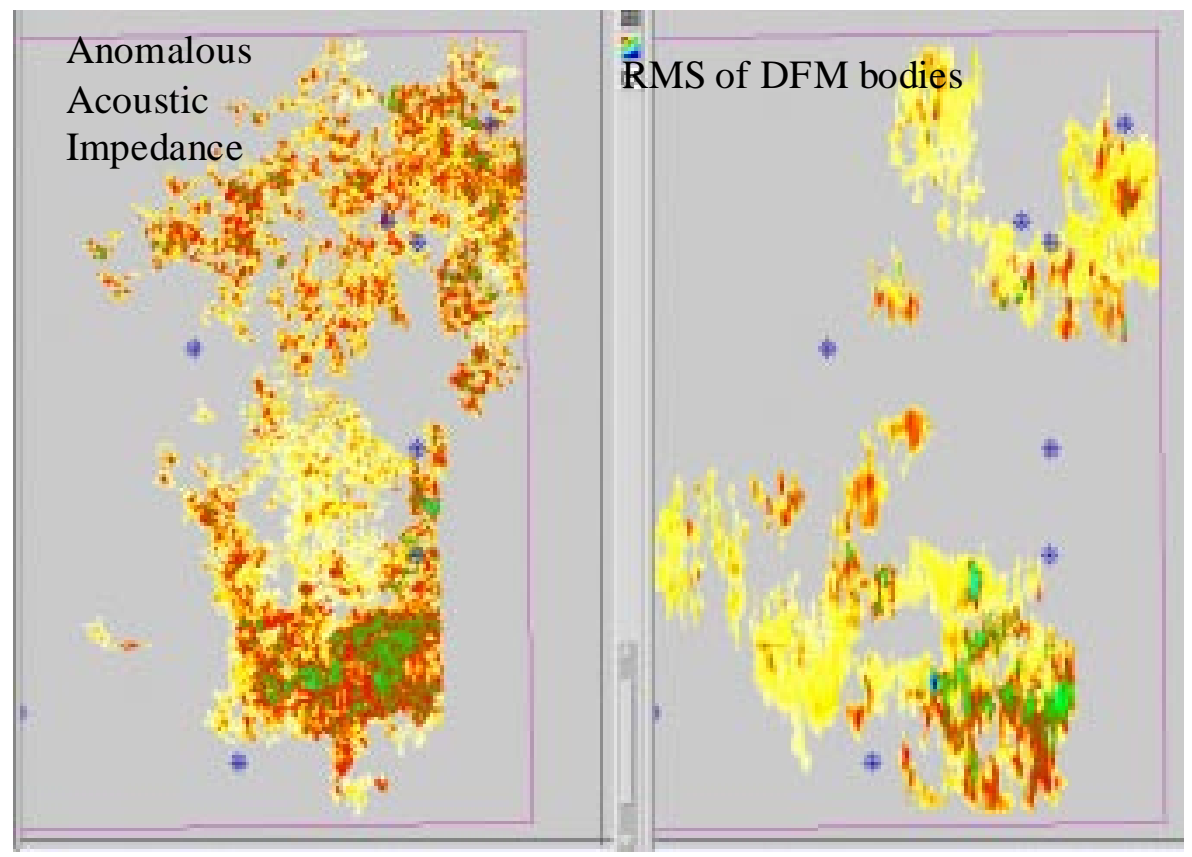

Figure 80: Comparison of the summed low-acoustic-impedance values and the DFM results.

\section{ONE-DIMENSIONAL SYNTHETIC SEISMOGRAMS GENERATED USING BACKUS AVERAGING}

We should always place a great emphasis on obtaining the best tie between synthetic seismogram and actual seismic data. Without this we cannot be very confident in interpretation results. In some environments it is difficult to obtain a reliable synthetic tie. There may be a variety of reasons why this happens. In our data set the conventional synthetics does not tie the data well. Hence, one of our goals is finding a solution for the problem and obtaining a simple and complete workflow for creating a good synthetic seismic tie which we can use on all the data routinely.

In the Wamsutter field we encounter the presence of extremely low-velocity thin beds and the lack of a checkshot survey. Thickness of these beds varies from 3-5ft to a maximum of $20 \mathrm{ft}$. The beds are rich organic coals interbeded with shales and very tight sandstones. Average porosity in these sandstones is 5-10 percent (Figure 60). Although no checkshot is available for any of the wells in the survey, we are able to convincingly tie one regional reflector to the base of the coal sequence (Figure 81). However, the remaining reflectors do not readily tie. We chose to investigate this further, by dealing with the problem of upscaling.

Seismic measurements respond to petrophysical properties at a larger scale than $\log$ measurements. It is impossible to obtain detailed, high-resolution petrophysical reservoir models from bandlimited seismic data. Seismic provides information on the scale of several tens of feet; $\log$ measurements on a scale of feet at the most. Scaling the effect of the interbedded coals and sandstones from the logging scale (used for creation of a synthetic seismogram) to the seismic scale may account for most of the mis-tie observed. 

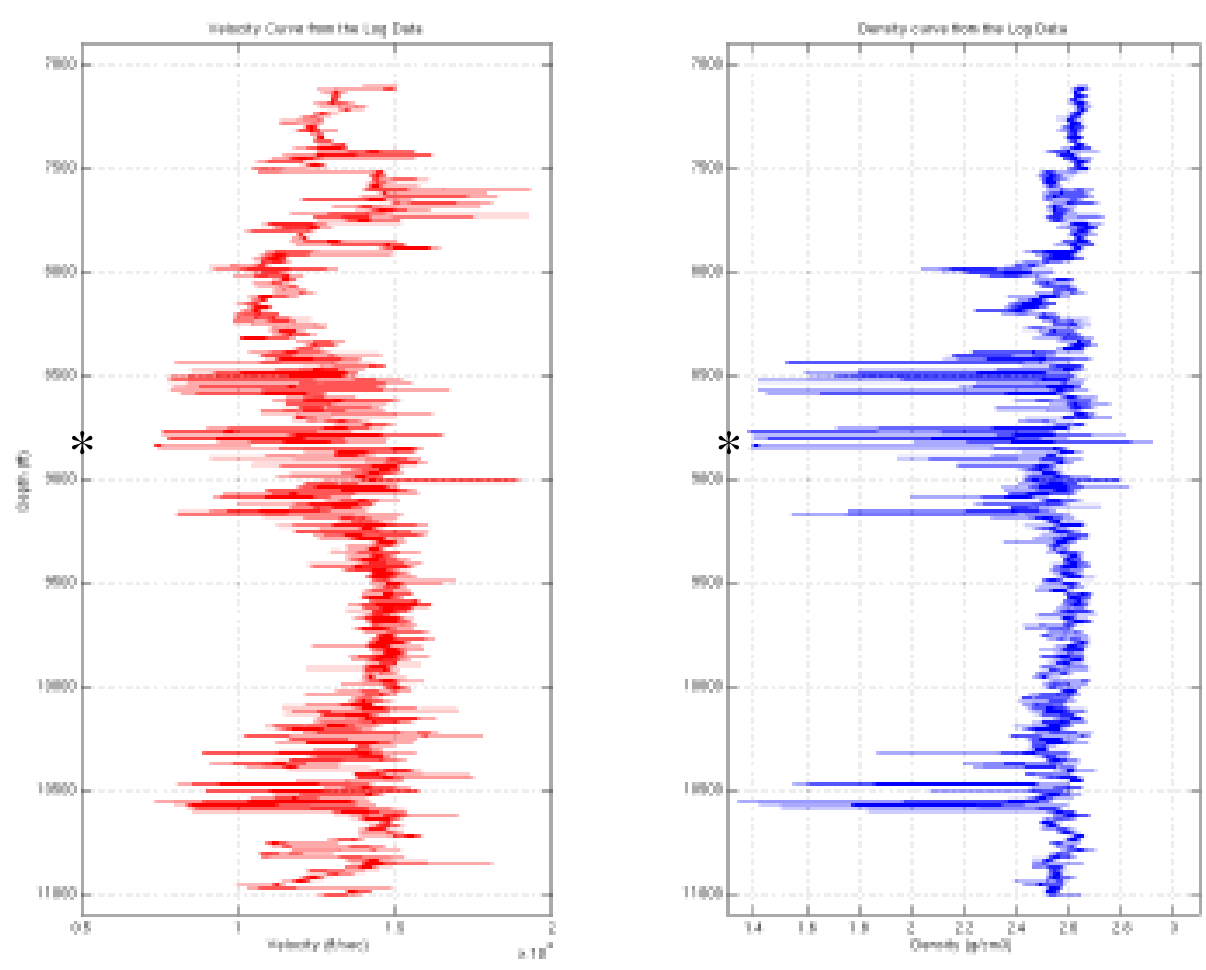

Figure 81:

Velocity and

Density logs.

The only reflector

that is clearly

identifiable on

these logs is the

base of the

Almond (*), at the

deepest coal, recognized by the low density.

\section{Short Summary of Ray Theory and Effective Medium Theory}

Wave propagation in a layered medium depends on wave frequency, layer thickness and rock properties (Backus, 1962; Marion et al., 1995; Mukerji et al., 1995). Marion et al., 1995 performed an experiment where they measured the P-wave velocity through stacks of plastic and steel disks. We will briefly describe a thought experiment based on these published results in order to provide an understanding of upscaling. First, a stack consisting of only steel disks is placed between two transducers, and the P-wave propagated through the stack is recorded. Velocity of this steel "sandwich" is simply found by dividing the recorded time by the thickness of the stack. Now, we add to our steel stack an equal number of plastic disks, resulting in a thick stack of plastic and steel disks. Now the P-wave propagated through the stack consisting of a thick steel layer and thick plastic layer is recorded

We will find that the velocity through the stack of steel and plastic disks, is given by

$$
\frac{1}{V}=\frac{1 / 2}{V_{\text {steel }}}+\frac{1 / 2}{V_{\text {plastic }}} \text { (2). }
$$

This is a time-weighted average, and is appropriate whenever the wavelength of the sonic pulse is short compared with the "layer thickness'. Expression 2 can be generalized to

$\frac{1}{\mathrm{Vrt}(\mathrm{n})}=\sum_{i=1}^{n} \frac{f_{i}}{\mathrm{~V}_{\mathrm{i}}}$

where $f_{\mathrm{i}}$ are the volume fraction of the constituents (equal to the fractional layer thickness), $\mathrm{V}_{\mathrm{i}}$ are the individual velocities and $\mathrm{V}_{\mathrm{rt}}$ is the velocity based on ray theory. The resulting propagation time is 
$\operatorname{Trt}(\mathrm{n})=\frac{\sum_{\mathrm{i}=1}^{\mathrm{n}} l_{i}}{\operatorname{Vrt}(\mathrm{n})}$

where $l_{\mathrm{i}}$ is the thickness of each layer (Rio et al., 1996). In this case velocity is the average velocity of the stack containing different layers. As long as the wavelength of the sonic pulse is short compared with the layer thickness, the velocity is always the time-weighted average of the velocities of the disks. The above thought experiment describes ray theory.

Now we consider another experiment using the same setup (the stack of equal parts of steel and plastic disks) and obtain measurements of the static bulk modulus and shear modulus by slowly compressing and rotating the stack of disks. The average, or composite, bulk and shear moduli are found by averaging their inverses - that is the compressibility (inverse of bulk moulus) is averaged over the various layers, and the bulk modulus obtained from the average compressibility. Likewise, this composite P-wave modulus $(\mathrm{K}+4 / 3 \mu)$ is found by averaging its inverse $[(1 /(\mathrm{K}+4 / 3 \mu)]$, where $\mu$-shear modulus or rigidity; K-bulk modulus or incompressibility. We can determine velocities using linear elasticity and the overall bulk and shear moduli of the stacked media:

$\mathrm{V} p=\sqrt{\frac{\mathrm{K}+4 / 3 \mu}{\rho_{\mathrm{b}}}}$ (5) and $\mathrm{V}_{\mathrm{s}}=\sqrt{\frac{\mu}{\rho_{\mathrm{b}}}}(6)$,

where Vp-compressional-wave velocity; Vs-shear-wave velocity, and $\rho_{\mathrm{b}}$-bulk density.

We should use the composite $\mathrm{P}$-wave modulus calculation of $\mathrm{V}_{\mathrm{p}}$ for extremely long wavelength sonic pulses (compared arithmetic layer thickness). Hence, we do not just average velocities for very long wavelength; we average inverse square of velocities multiplied by density in order to obtain the long-wavelength velocity $\mathrm{V}_{\mathrm{EMT}}$

$$
\frac{1}{\rho \mathrm{V}^{2} \text { EMT }}=\frac{l i / 2}{\rho \mathrm{V}_{\text {steel }}^{2}}+\frac{l i / 2}{\rho \mathrm{V}_{\text {plastic }}^{2}}
$$

The only difference between these two experiments described above is that we are using different wavelengths. Ray theory model uses very small wavelets or extremely high frequencies. On the other hand, the static compression experiment uses very long wavelengths or low frequencies and is equivalent to the effective medium theory.

The overall effect of using the effective medium theory is the enhanced importance of thin lowvelocity layers. Slower layers are weighted more than in ray theory, because the slowness (inverse velocity) of each layer is squared before averaging; the slownesses themselves are averaged in ray theory.

The transition from effective medium theory to ray theory occurs at $\lambda d \sim 10$ to 20 . In other words, when the wavelength is 10-20 times larger than the layer thickness, use of effective medium theory is appropriate. If the wavelength is less then 10 times of the layer thickness then ray theory should be used. Other investigators have found slightly different relationships but all found a transition at $\lambda d \cong 5-20$ (Hovem, 1995). 
When we tie log data to seismic data, we generally create a synthetic seismogram from the sonic and density logs, and then compare this with the (surface) seismic data. In general, there is a disagreement between the time-depth relationship obtained by directly integrating the sonic log and that found from a checkshot survey or vertical seismic profile at the well; or with the timedepth relationship required to obtain a satisfactory match between the synthetic and recorded seismograms. The discrepancy, or 'drift' is usually eliminated by calibration or correcting the sonic-velocity values by an appropriate amount. There may be many causes for this disagreement, such as invasion effects on the sonic log, but here we will investigate the effect of fine-scale layering and practical approaches to implementing an effective medium theory model in synthetic seismogram generation, hoping to avoid the sometimes arbitrary correction applied by stretching and squeezing the synthetic seismogram.

\section{Our Approach}

A summary of time-depth functions, velocity functions, and the scenarios investigated in this paper is introduced in Figure 82. The individual terms are explained in the text as needed.

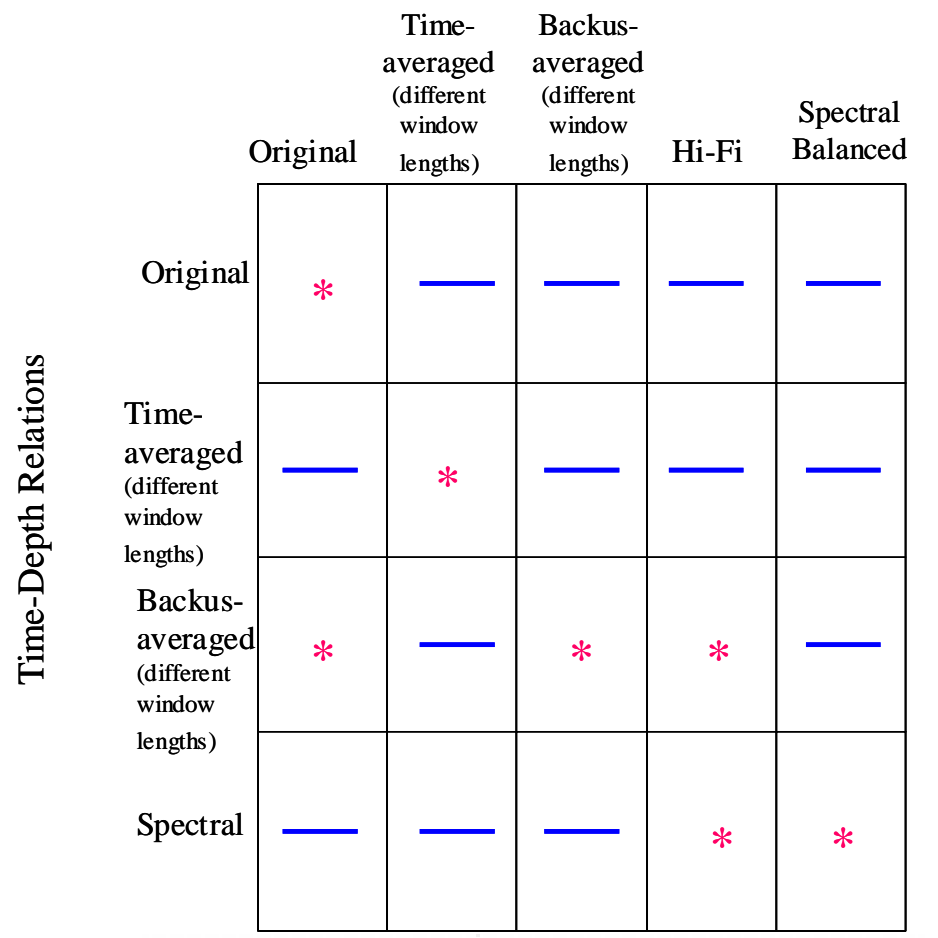

Core D delim 1 as Das Denuly

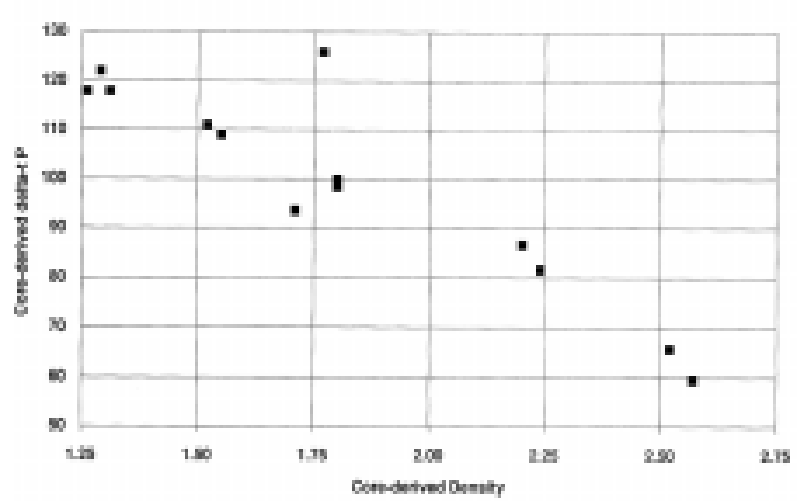

Figure 82: Table of scenarios investigated for upscaling.

Various terms will be defined as needed in the text.

We chose one typical well from our data set, containing thin interbedded coals, lowporosity sands, and shales. Because log data obtained in coal layers is often unreliable due to hole conditions, we compared our logged data with laboratory and $\log$ observations on coal in New Mexico (Figure 83, Pennington, 1993). Our data follows the same ranges and trends, and we believe that our well log data is reliable and not significantly affected by logging tool errors or borehole environment (See Figures 60 and 81).

Figure 83: Relationship between density and velocity in coals, based on core

measurements.

We used the Backus (1962) averaging technique to account for thin layers. For normal incidence, this is equivalent to the formulation presented earlier. We do not have available to us pre-stack data or any other shear wave information and therefore 
we averaged inverse of the compressional-wave modulus $\mathrm{M}$

$\mathrm{Vp}=\left(\mathrm{M} / \rho_{\mathrm{b}}\right)^{1 / 2}(8) ; \mathrm{M}=\left(\mathrm{K}_{\mathrm{b}}+4 / 3 \mu\right)(9)$.

We calculated $\mathrm{P}$-wave moduli $\mathrm{M}$ for the entire well from the sonic and density logs, and then found the harmonic mean of P-wave moduli M over certain averaging window (Figure 84).
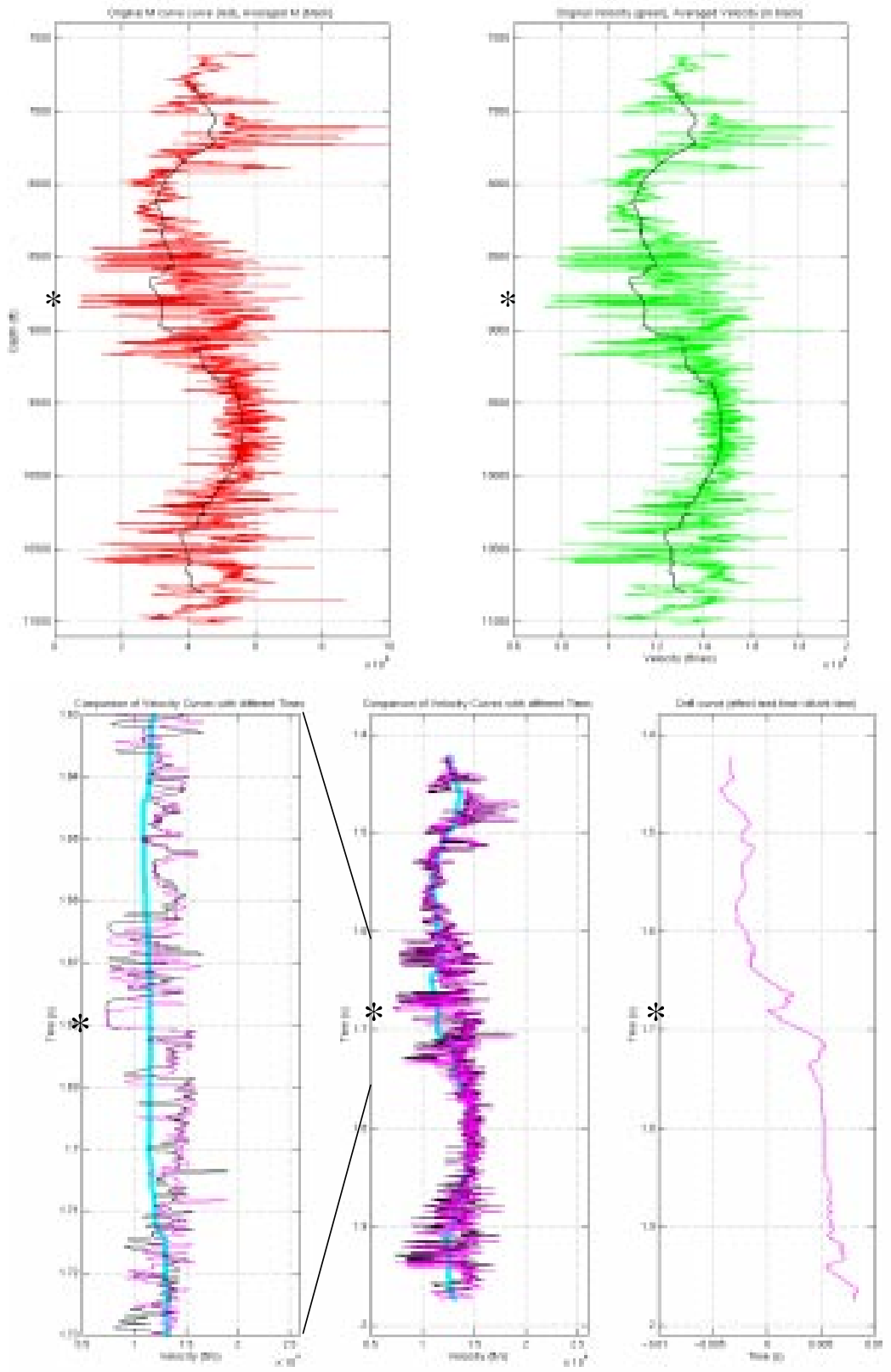

Figure 84: Calculated modulus (left side, red) and Backus-averaged values (black); computed velocity (right side, green) and Backus-averaged (black).

The arithmetic mean of density was found using the same window size. Finally, a new smoothed Backusaveraged velocity was obtained using these values:

$$
\begin{aligned}
& \bar{V}(z)=\sqrt{\bar{M}(z) / \bar{\rho}(z)} \\
& \bar{M}(z)=\left[\frac{1}{n} \sum_{z=1}^{n} \frac{1}{M(z)}\right]^{-1}(11) \\
& \text { and } \bar{\rho}(z)=\frac{1}{n} \sum_{z=1}^{n} \rho(z)
\end{aligned}
$$

At every point, the new Backus-averaged velocity function $\overline{\mathrm{V}}(\mathrm{z})$ represents the local (over the averaging window length) effective medium equivalent of the original layered medium (Figure 84). Now we can estimate the new Backusaveraged time-depth relations from this new locallyaveraged velocity (Figure 85).

Figure 85: Comparison of original and Backus-averaged time-depth relations.

New time-depth relations were obtained from the Backus-averaged velocities. Original velocity values are shown on the new time scale (left panel, magenta), Backus-averaged velocity - Vharm is in cyan. Original velocities are displayed on the original time-depth scale (left panel, black). Drift is shown on the right panel. It is the difference between original and Backus averaged times. Notice that drift is zero at the tying point, marked by the star *. 
In our data set, we can only fix one time-depth pair, by associating the base of a coal sequence on the log to a particular pronounced regional reflector on the seismic data. This is equivalent to having only one check-shot value, at a specific depth and represents a worst-case scenario for most exploration or development targets. The drift curve (Figure 84) is therefore set to zero in the middle of the panel at the single tie point. Drift is defined as a difference between the time (at any depth) obtained from the averaged velocity profile and that obtained from the original sonic log; a positive slope (increase in drift with depth) implies that the averaged velocities are lower than the original velocities. Well logs are uniformly sampled in depth with a sampling rate of $0.5 \mathrm{ft}$. In time domain, however the sampling will be not uniform. We interpolated on our data in the depth domain, keeping the frequency range of our data unchanged, with new depth increment of $0.05 \mathrm{ft}$. After applying the time-depth relationship we had obtained, we resampled our log in time to $0.00002 \mathrm{sec}$. The sample rate of our seismic data is $0.002 \mathrm{sec}$.

In order to directly compare our Backus-averaged results with ray theory results, we calculated the arithmetic mean (using the same window size) of the sonic log, and than converted the averaged sonic values to velocity, yielding time-averaged time-depth relations (Figure 85).
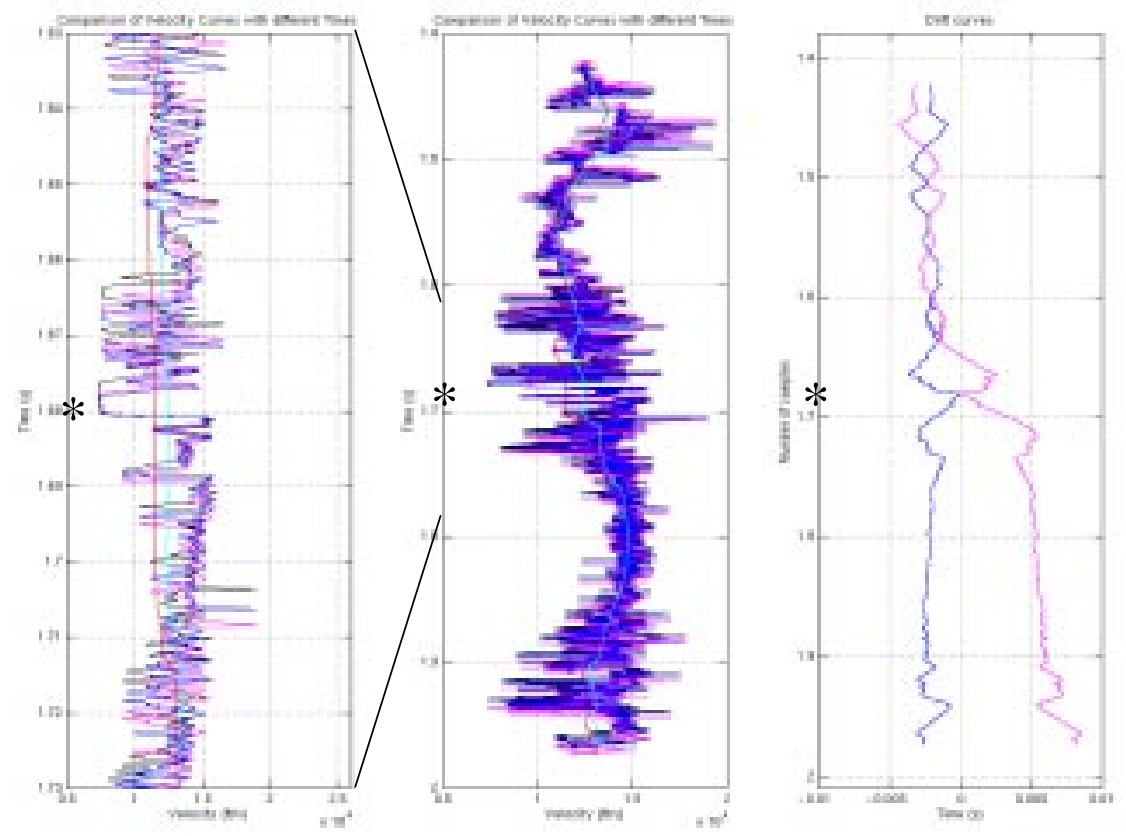

Figure 85: Additional time-depth relations. Original velocities are plotted in the middle and left panels using these time-depth relationships: Blue: arithmetic mean; Magenta: harmonic mean; Black: original sonic Smoothed velocities Vharm (red) and Varith (cyan) are also shown. Right panel: Drift curves (blue corresponds to time difference between original and averaged ray theory times, magenta is time difference between original and Backus).

Having obtained a Backus-averaged time-depth relationship, we have several options for implementing a new synthetic seismogram routine. First, which velocities should we use to construct our new reflection-coefficient series? Should we use the original logged values or the Backus-averaged velocities to construct the new reflection-coefficient series, or should we modify the original sonic log to better portray the values after Backus averaging? We will investigate these possible approaches starting with the last.

\section{"High-Fidelity Backus Averaged" Velocity Function}

To see if the use of Backus-averaged velocities (not just their time-depth relationships) in construction of new reflection-coefficient series will improve our synthetic-seismic tie, we found the ratio of two velocities, Backus-averaged velocity and time-averaged velocity at each point in 
time. Then we multiplied the original velocity by that ratio and obtained a new "High-Fidelity Backus Averaged" velocity curve (Figure 87). The ratio of velocities is lowest in thin-bedded areas. Thus we added some "amplitude" or emphasized the areas of thin beds more on the original sonic log. In effect, we used the new Backus-averaged velocities to emphasize the lowvelocity beds, while retaining the high-frequency transitions at bed boundaries. The integrated transit time of the Hi-Fi Backus averaged log will yield the Backus-averaged time-depth relationship.
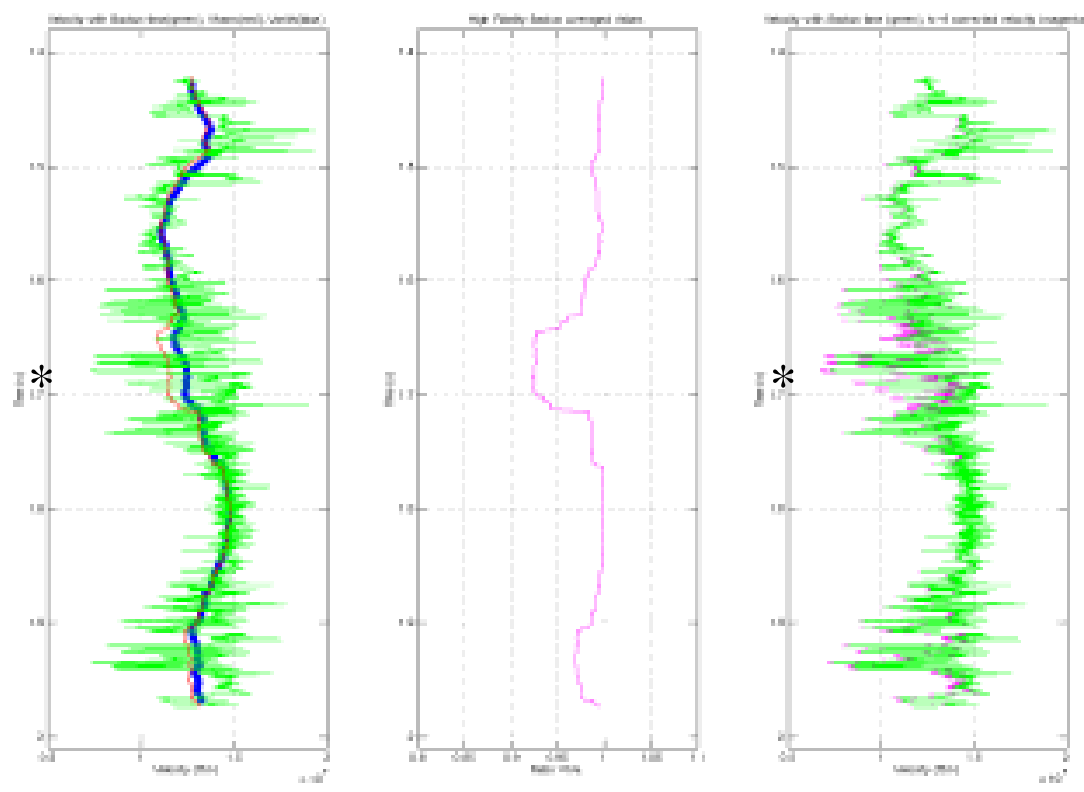

Figure 87: "High-Fidelity" Backus-averaging. Left panel shows the original velocity (green), Vbackus (red) and Varith (blue). Central panel shows he ratio of Backusaveraged to arithmetic-avraged velocities. Notice that the arithmetic averaged velocities are everywhere higher than Backusaveraged velocities. To obtain the "High-Fidelity" Backus-averaged curve we multiplied original velocity (green curve on the right) by that ratio (magenta curve).

This display uses the Backusaveraged time-depth relationships.

We can see that there are different ways to compute a new upscaled velocity function (Figure 84) using any of the following combinations: original velocity and density logs with original timedepth relations; Backus-averaged velocities and resulting Backus-averaged time-depth relations for different averaging window lengths; Hi-Fi Backus-averaged velocity logs; and time-averaged velocity logs with corresponding time-averaged time-depth relationships. We now turn to comparing the window lengths used.

\section{Window length}

So far we have concerned ourselves with a single window length $(400 \mathrm{ft})$ for averaging. This window corresponds to a full wavelength at a frequency of $33 \mathrm{~Hz}$ and velocity of 13,205 ft/sec, for example. But what window length is optimal if we use just one window size for our averaging? Should it be equal to the full wavelength, half of the wavelength, quarter of the wavelength, two wavelengths, ten wavelengths; or perhaps the window length should be connected to the average layer thickness? Because the size of the wavelength typically changes with depth, should we use a dynamically changing operator with depth? Relations between wavelength and frequency for average velocity function and sands and coal velocities are displayed in Figure 88. 


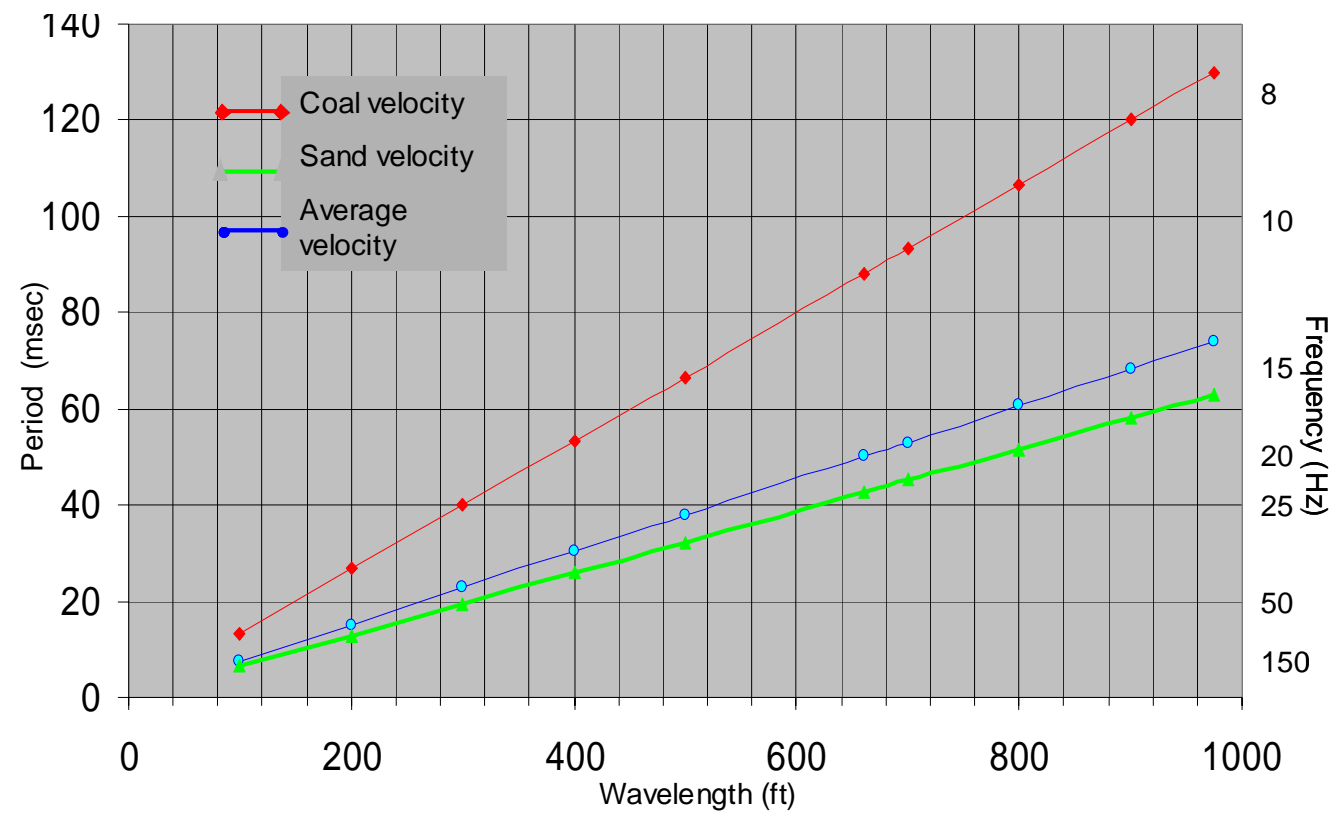

Figure 88:

Relationship between period or frequency and wavelength.

Now we can compare the layer thickness $(d)$ and the wavelength $(\lambda)$. Frequency spectrum of seismic data ranges from 20 to $60 \mathrm{~Hz}$, corresponding to wavelengths of 660 and $200 \mathrm{ft}$, respectively. The thickness of the layers defined from the well log data varies between 3 to $12 \mathrm{ft}$ approximately. $\lambda d$ for these ranges extend from 17 to 220 and fall into the effective medium theory range $(\lambda d>10)$. The only case we studied that falls into the ray theory range is the original time-depth relationship with original velocities.

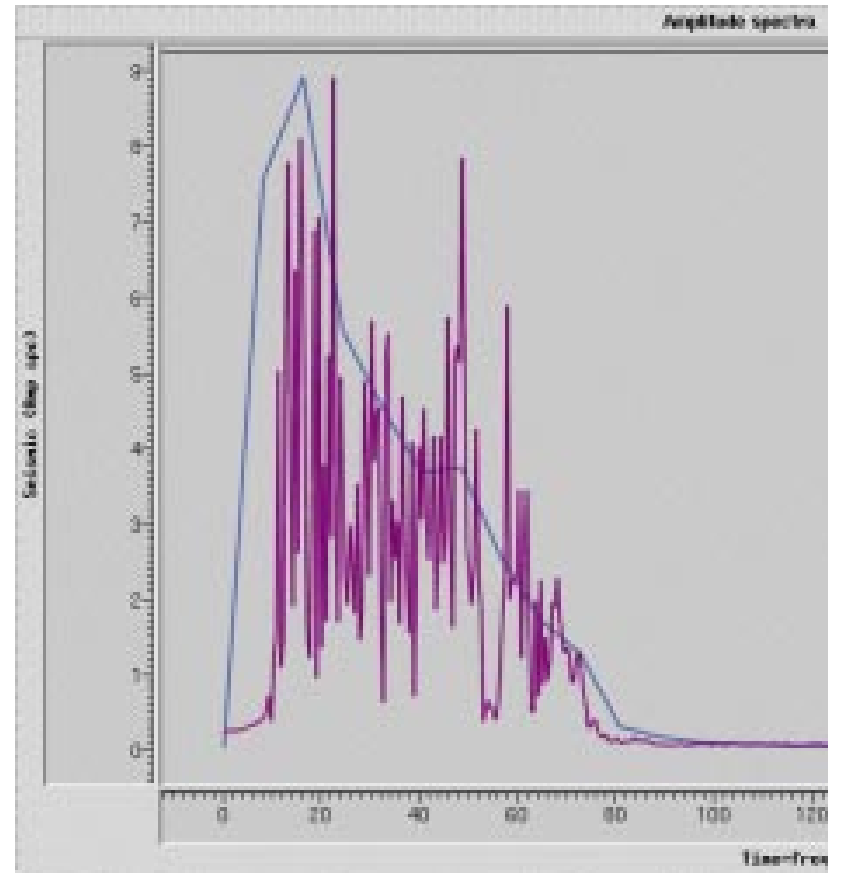

Figure 89 shows seismic bandwidth at the well location within the time interval $1 \mathrm{sec}-2 \mathrm{sec}$. There is no clear "dominant" frequency in this spectrum. We developed a method to account for the range of frequencies present in our wavelet, using different window length.

Figure 89: Amplitude spectrum (purple) of seismic data at a well location, 1000-2000 ms. Estimated wavelet spectrum (blue).

\section{Spectral Balanced Velocity Function}

For each window length we can find the corresponding frequency (assuming a velocity) (Figure 88). We obtained a wavelet that best fit the seismic data, using a conventional synthetic seismogram calculation, allowing the conventional, somewhat arbitrary, stretching and squeezing (Figure 89). We then used an amplitude weighting for different window lengths based on the relative amplitude of wavelet at each corresponding frequency (assuming the 'average' velocity). We generated a suite of smoothed Backus-averaged velocities for a range of window sizes, and then normalized each velocity function according to the weighting assigned, 
and added these weighted curves together in the depth domain to obtain one "spectrally balanced" velocity function (Figure 90). Then we used this velocity function to obtain new timedepth relations. Results are discussed below.
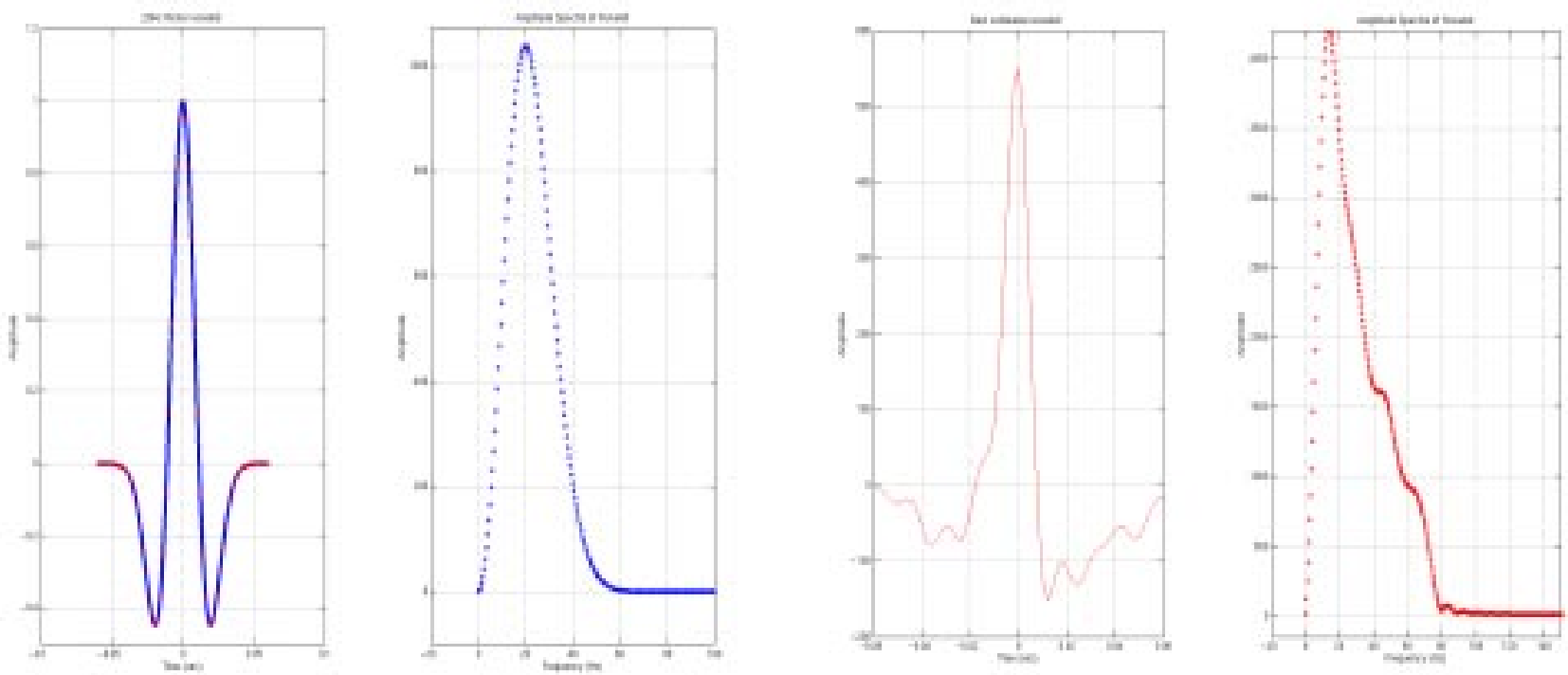

Figure 89: Wavelets used for synthetic seismograms and spectral balanced velocity functions. Correlation coefficients obtained for synthetic seismograms generated using "Best estimated wavelet" (red) showed higher correlation than those generated using $20 \mathrm{~Hz}$ Ricker wavelet (blue). Power spectrums of both wavelets have different frequency ranges and characters.

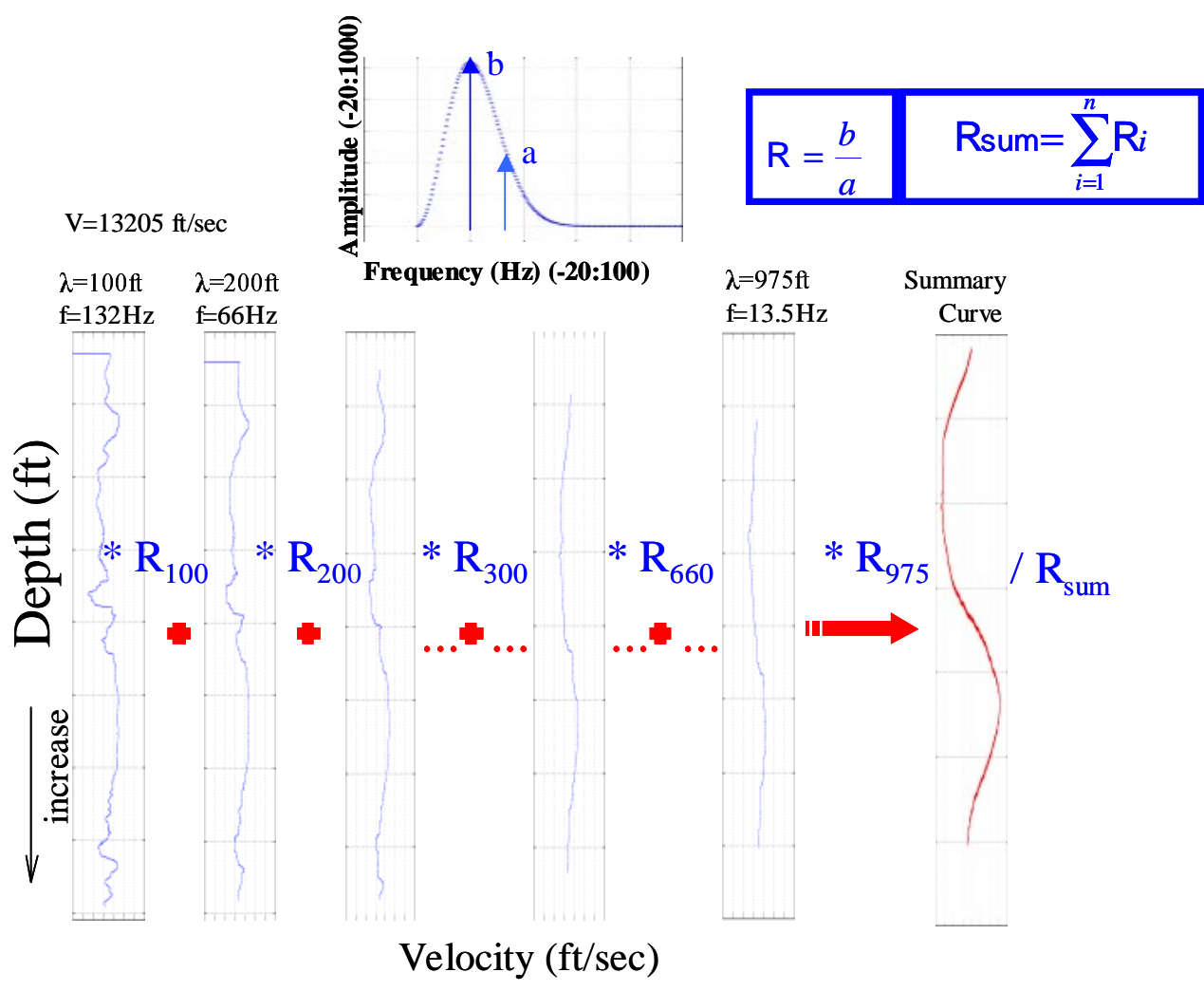

Figure 90: Spectrally balanced velocity function. Each wavelength correspond to certain frequency (average velocity. Using the power spectrum of the best wavelet, coefficients for each frequency (Rn) are found. Smoothed Backusaveraged velocities are normalized by $R$. All weighted Backusaveraged velocities are added together. 


\section{Results}

In order to compare the effects of the various averaging techniques, window lengths, and velocity functions, we investigated two specific measurable results. First, we compared the drift curves of the different techniques, noting the importance of various high-velocity and lowvelocity beds on the time separation between certain markers. Then, we computed synthetic seismograms for a number of the cases, and compared them with the seismic trace recorded nearest the well, finding the correlation coefficient between the synthetic and actual trace.

\section{Drift Curve Comparison}

The time-depth function that results from Backus averaging of thin beds is, in general, slower than the time-weighted averaged (ray theory) function - that is, bed separation will tend to increase in time for the Backus averaging. This occurs because the slowness are squared, then averaged, for Backus averaging, rather than simply averaged as in the ray theory, and the slower layers therefore obtain greater significance. For a 'static' experiment, such as the one described earlier, where a layered medium is compressed, the average is taken over the entire stack. But for an earth model, with thin layers buried at thousands of feet depth, and sonic pulses with wavelengths less than several hundred feet, we need to determine the appropriate averaging scheme and scale length. We can compare the different time-depth relationships by comparing their drift curves.

The drift curve shown in Figure 85, compared the time-shift at such point in two-way time between the original integrated sonic log and the 400-ft windowed Backus average. A positive trend (an increase in time shift with increasing two-way travel time) indicate a longer travel time for the Backus averaged function. Note that if we compared a Backus-averaged log with a ray theory log that was averaged over the same time window, the drift curve would always have a positive (or zero) trend, because of the greater significance of low-velocity layers in the Backus average. On the other hand, the original integrated sonic curve is not averaged at all (beyond the $0.5 \mathrm{ft}$ sample separation) and the drift curve in Figure 85 shows some negative trends. These negative trends occur because the Backus-averaged log at some depth obtains a higher velocity then the original log simply because its window length happens to include a large fraction of high-velocity layers. Locally, therefore, the windowed Backus-averaged log may exhibit higher velocities then the original $\log$, but the overall trend is necessarily to slower velocities and position of drift trends.

Figure 91 shows the effect of Backus averaging within a small section of the log, demonstrating the effect of window length that incorporate increasingly greater fractions of high-velocity rocks surrounding a set of low-velocity layers.

Figure 92 shows a set of drift curves for different window lengths used in Backus averaging. In general, the shorter window lengths result in less drift, as might be expected. Locally, however, the longer window can result in significant negative drift trends, as shown from about $7900 \mathrm{ft}$ to $8300 \mathrm{ft}$ depth. In this instance the negative drift trend can be seen to be caused by the highvelocity layer at about 7600-7800ft depth, which influence the longer window lengths to considerably greater depths then the shorter window length. 


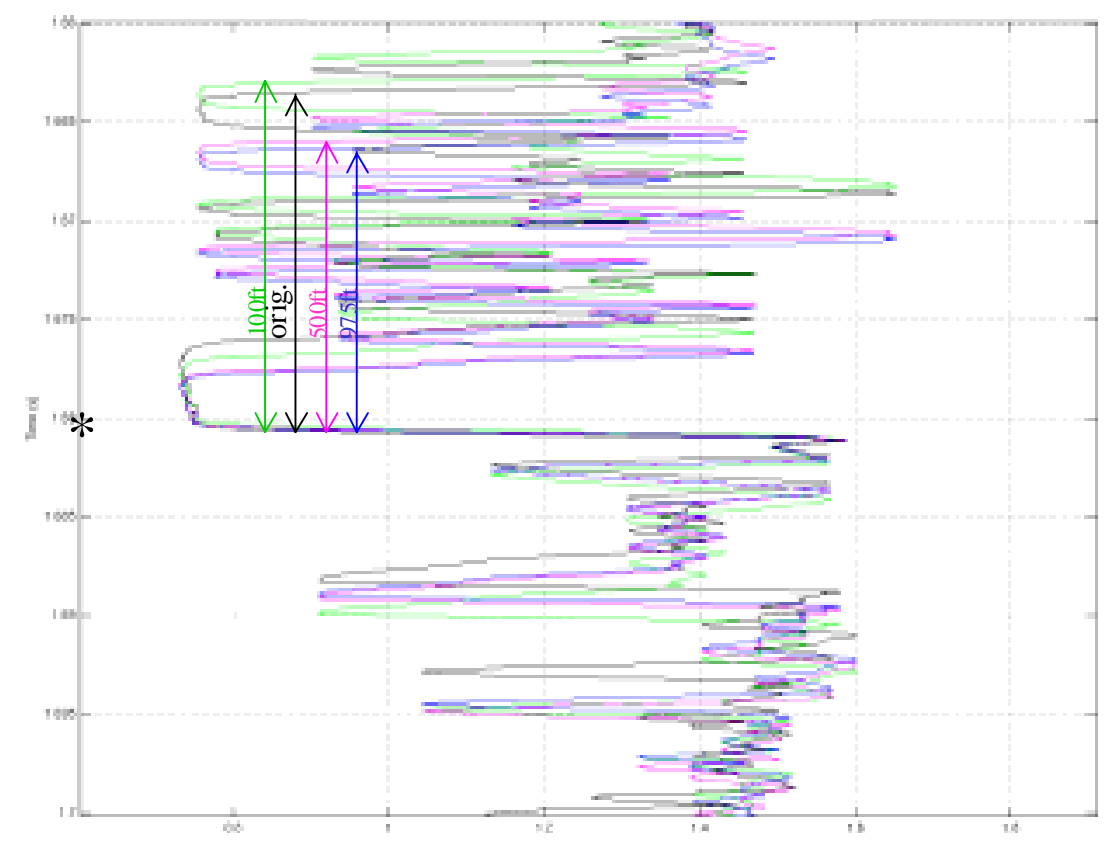

Figure 91: The effect of window length.

Original Velocity $\log$ is plotted using different timedepth relations, details near the tie point (shown): Black: original sonic; Green: Backus averaged time-depth relationship found using $100 \mathrm{ft}$ window; Magenta: Backus averaged time-depth relationship found, $500 \mathrm{ft}$ window; Blue: Backus averaged timedepth relationship found using $975 \mathrm{ft}$ window.
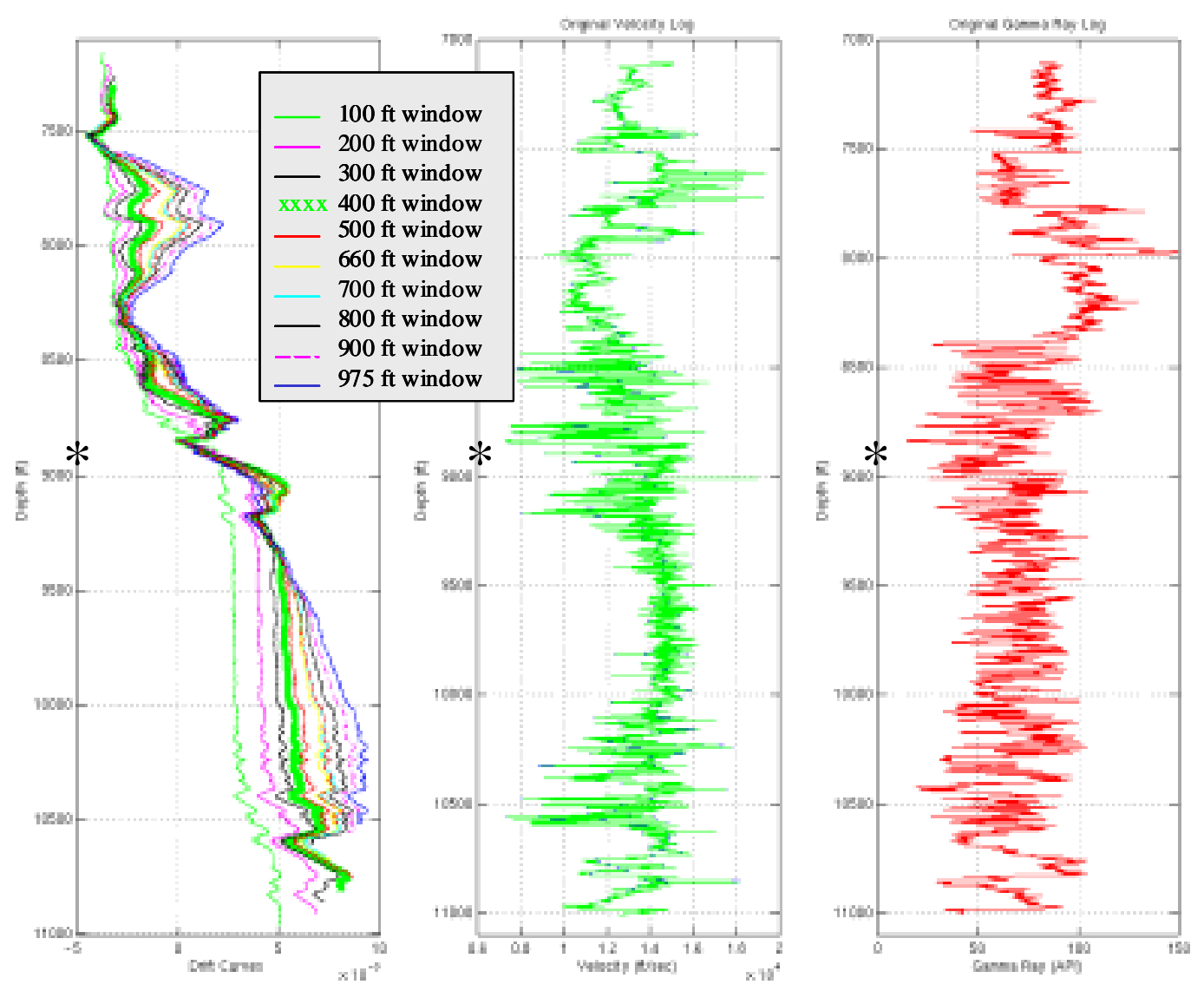

Figure 92: Drift curves corresponding to different window sizes (left panel).

Window sizes are shown in the legend window. Original velocity log (middle panel, green log) and original gamma ray log (right panel, in red) are shown. Marker corresponding to the only tie point is shown. 


\section{Correlation Coefficients}

In our data set, we do not have an independent estimate of drift through a checkshot survey or vertical seismic profile. In order to evaluate the various drift curves (and velocity functions) we obtained correlation coefficients between synthetic seismograms and the seismogram recorded nearest the well. Figure 93 shows that correlation coefficients for original (conventional) synthetic seismogram and seismograms created from the Hi-Fi Backus-averaged velocity logs have much higher correlation coefficients than those obtained from the original velocity logs. Different window lengths have little variation in correlation coefficient values and which is the best window length that should be used for Backus-averaging is not clear in this case.

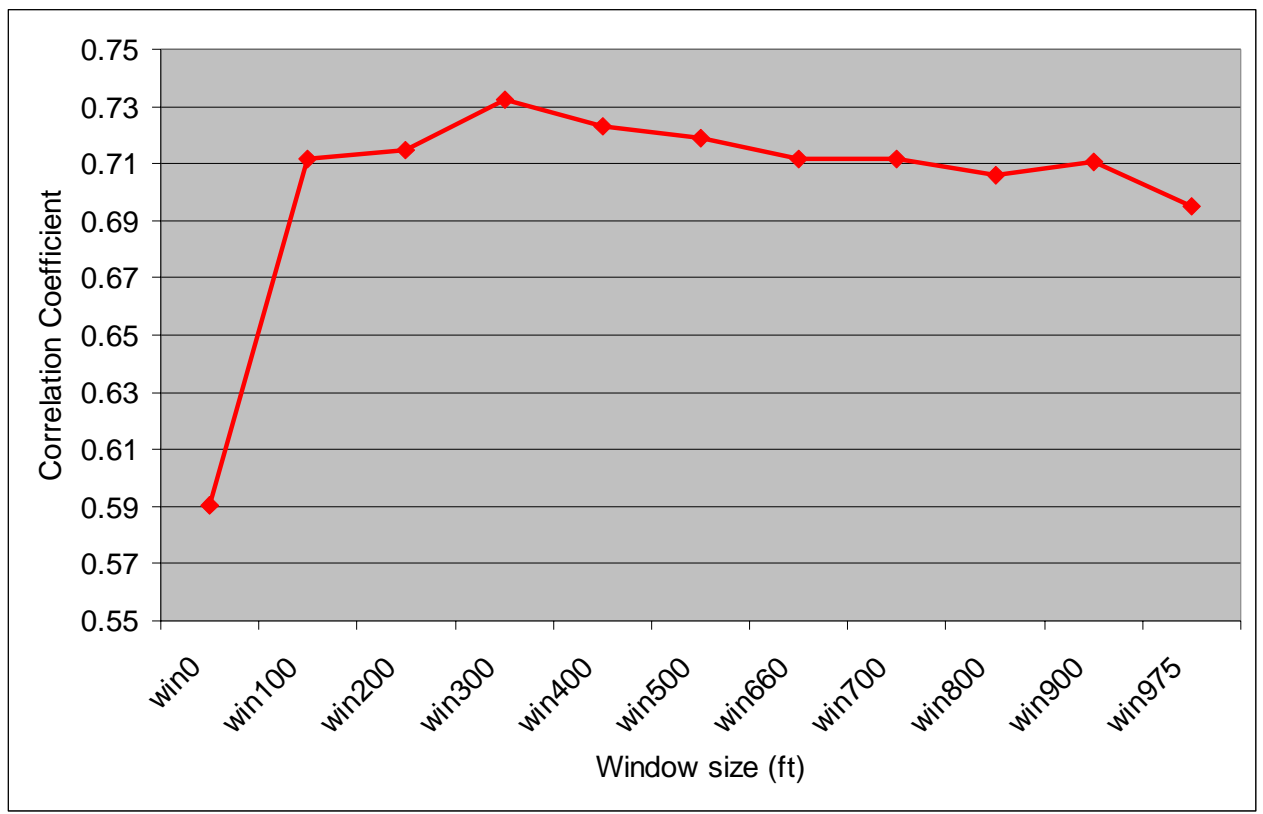

Figure 93: Correlation coefficients for synthetic seismograms from Hi-Fi Backus averaged velocity functions.

Figure 94 shows resulting synthetic seismograms. At first glance, it is difficult to see why there is such a big improvement in correlation coefficient values when all the synthetic traces look so similar.
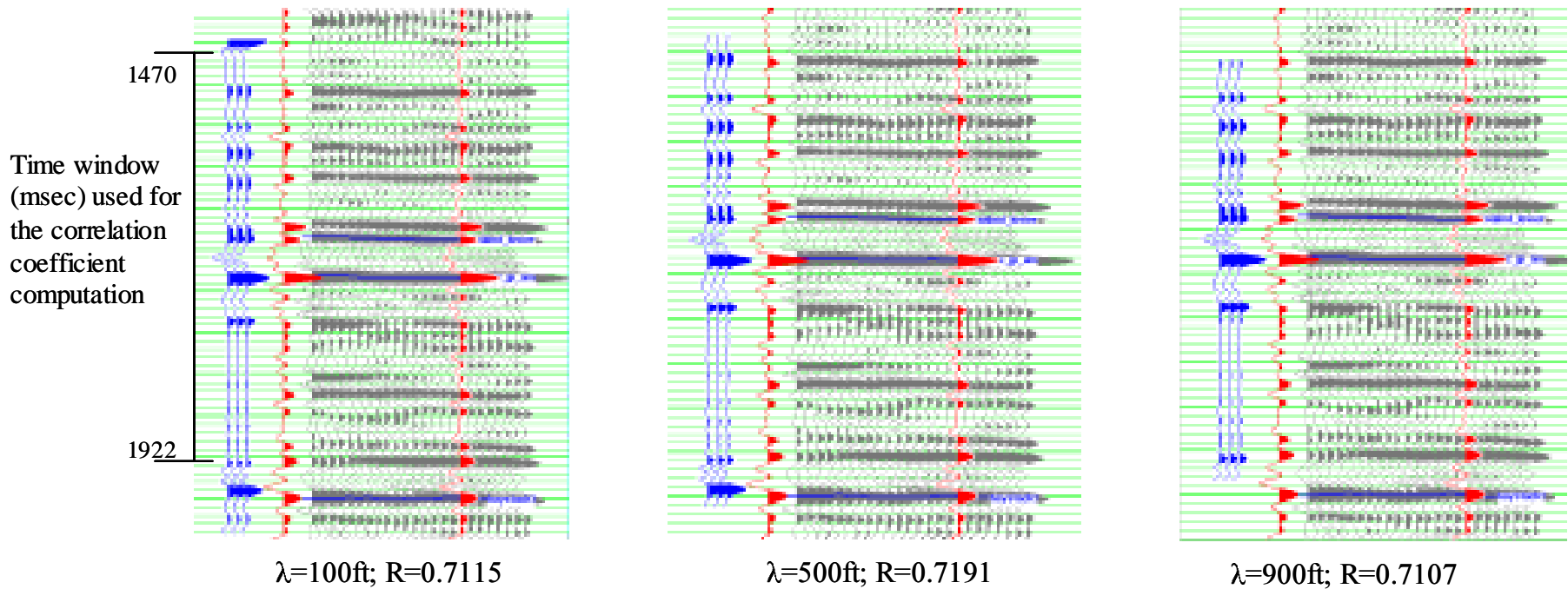

Figure 94: Synthetic seismograms (blue) generated using "high fidelity Backus averaged" velocity logs. 
Figure 95 zooms on two synthetic seismograms. One is created from original velocity log and another is created using Hi-Fi Backus-averaged velocity log with Backus-averaged time-depth relationships. Note that there is a difference in the peak alignment; peak amplitudes themselves differ too. The synthetic seismogram created using Hi-Fi Backus-averaged velocity log correlates with original seismic trace better, apparently due to an improved time-depth relationship

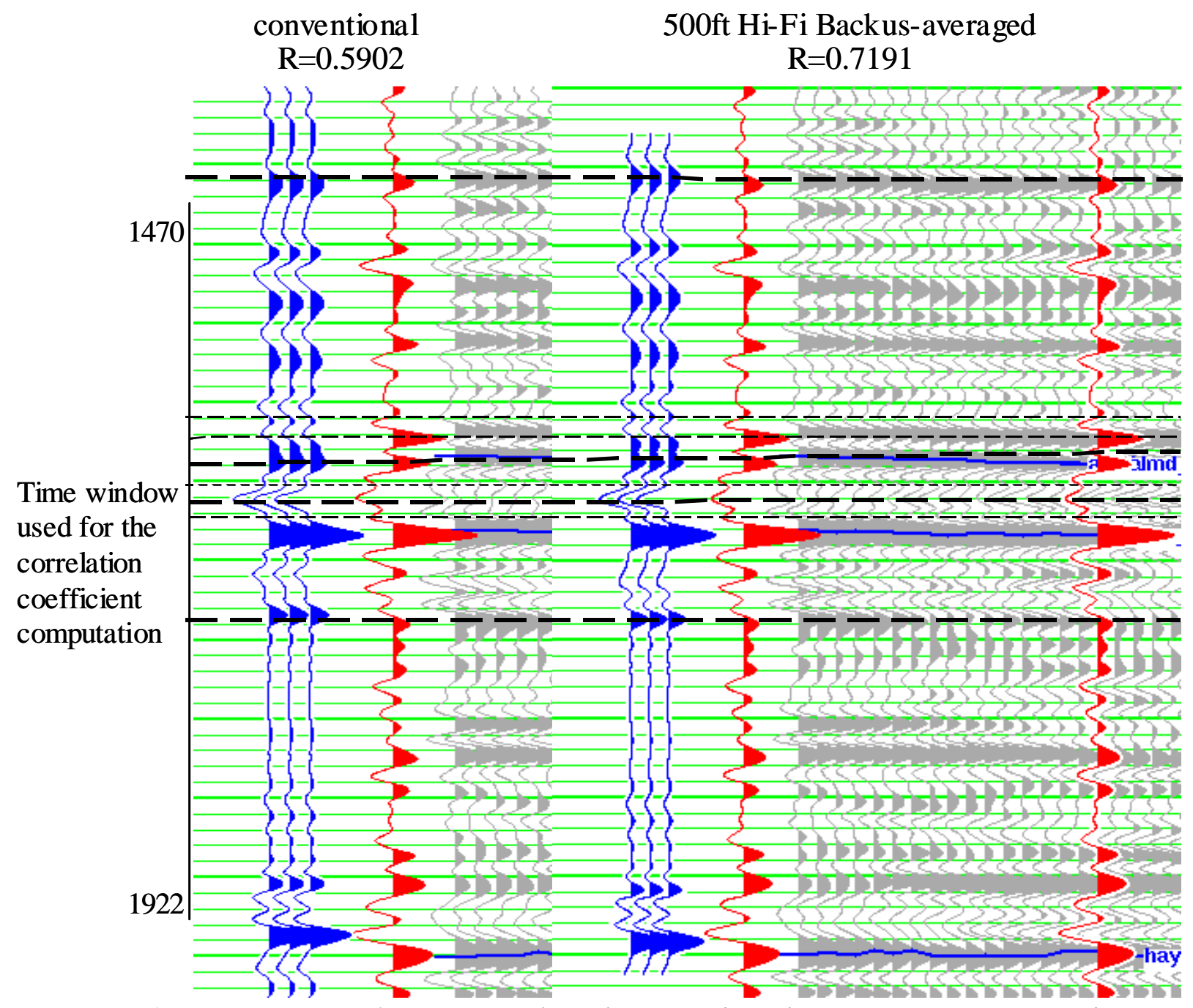

Figure 95: Comparison of conventional synthetics and synthetic seismogram created using Hi-Fi Backus-averaged velocity log. Synthetic traces are shown in blue, seismic trace in red.

\section{Conclusions}

We successfully performed upscaling of well data to seismic, created new velocity functions ("High-Fidelity Backus Averaged" velocity function and Spectral Balanced Velocity Function) which we might investigate further in the future. We compared drift curves of the different techniques and determined what causes unexpected behavior of drift curves. We determined that the correlation coefficient between seismic and synthetic traces improved after appropriate upscaling. 


\section{Teal South Time-Lapse Study, Gulf of Mexico}

The Teal South field lies offshore of Louisiana about 70 miles south of Morgan City. It is a fairly small field, until recently operated by Texaco, that produces from more than one horizon. Texaco chose that field for a time-lapse study because it was expected to deplete quickly and could provide useful information for evaluating time-lapse techniques within a few years. After their initial time-lapse study, Texaco offered the opportunity to study ocean-bottom multicomponent seismic time-lapse techniques to the industry in general, through a consortium organized by the Energy Research Clearing House (ERCH). That consortium grew to include a number of producing companies and service companies, as well as a few universities (additional information can be found on the ERCH web site: http://www.erch.org). Our involvement in the project, supported through this contract with the DOE, began as an effort to analyze the 'legacy' seismic-streamer data for the physical basis of the seismic attributes (this is a conventional 'bright-spot' play), and to predict the observations expected from the time-lapse studies. If we have calibrated the attributes properly, our predictions should be viable; if the predictions are incorrect, then we must not have calibrated the attributes appropriately. Our efforts quickly expanded to include seismic petrophysical aspects of some very surprisning observations in the time-lapse data.

An image of the difference volume found by Texaco in the first phase is in Figure 96.

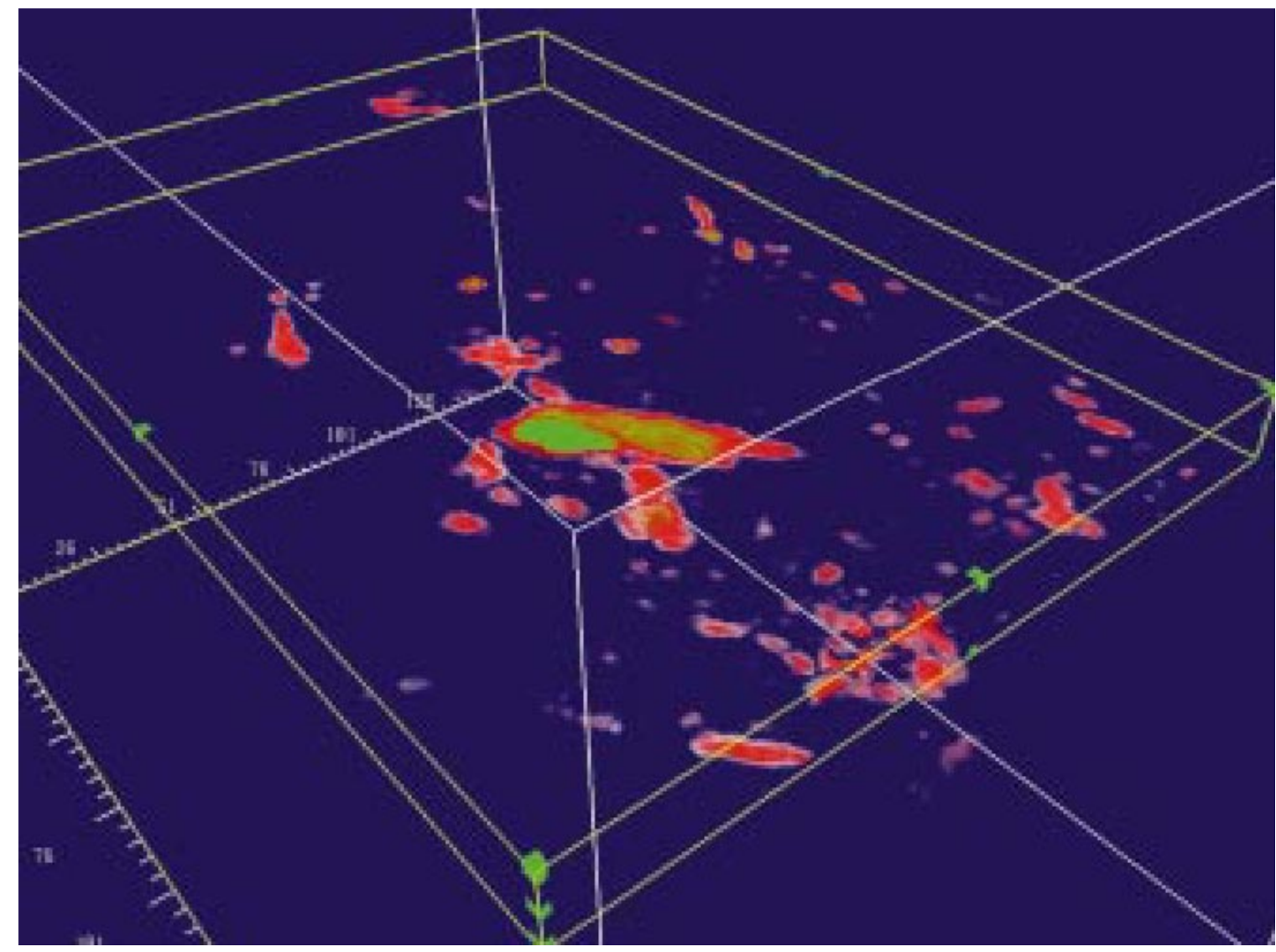

Figure 96: Three-dimensional image of a time-lapse difference volume found by Texaco during the first phase of the Teal South project. Bright colors indicate a change in reflection amplitude. From Ebrom et al, 2000. 
One of the unique aspects of our study of this field involved the use of the acoustic impedance volume (shown in Figure 97) to estimate rock properties in the oil zone and in the water zone of the reservoir formation. By making these two estimates, the 'dry-frame' properties of the formation could be inferred, assuming the Gassmann model. Then, using a reasonable pressuredependence for dry-frame velocities (described in a later section of this report), the reflectivity of the beds during production could be predicted. The results were surprising.

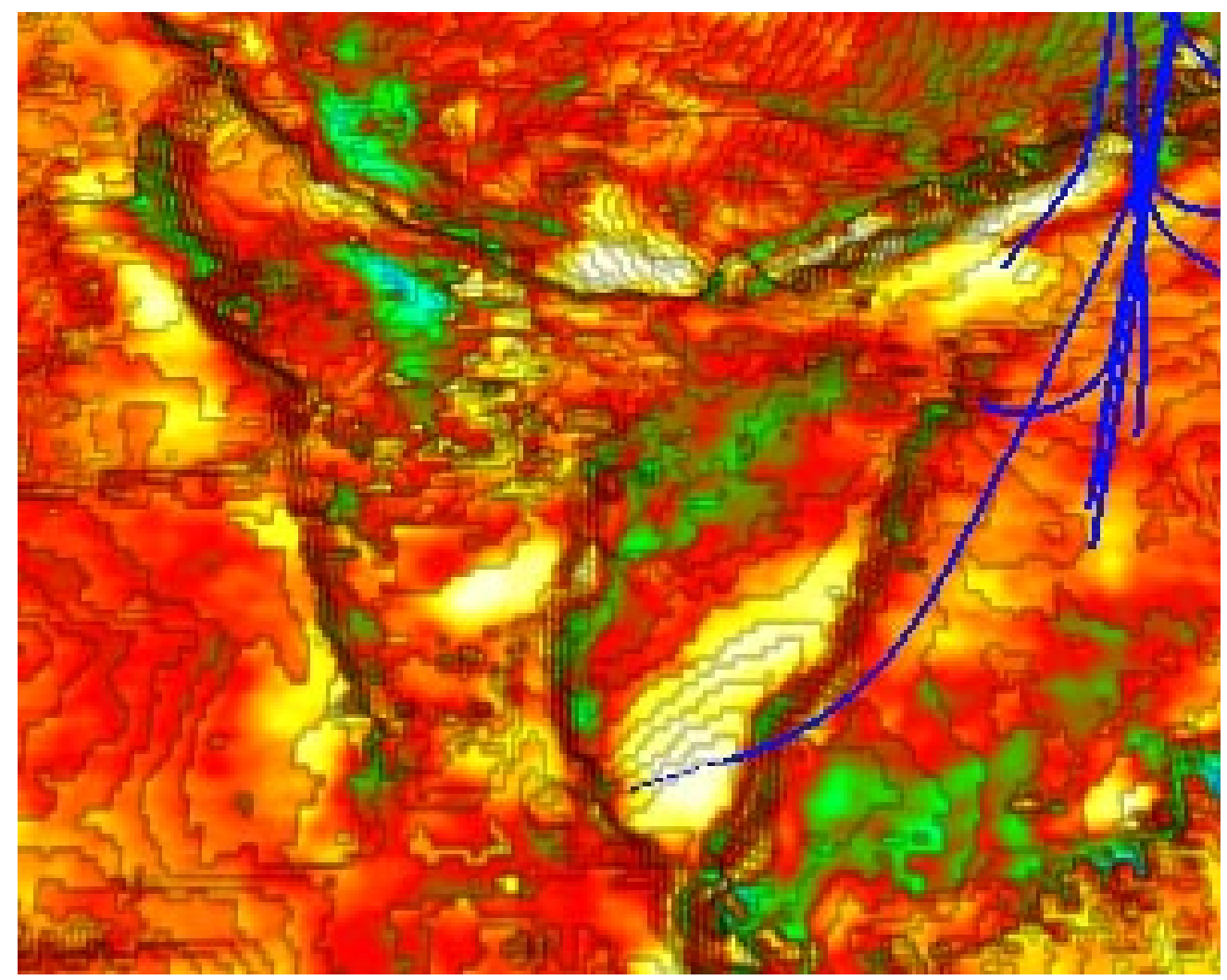

Figure 97: Acoustic impedance map view of the target horizon at Teal South.

\section{INTRODUCTION AND FIELD CHARACTERISTICS}

Geophysicists conduct time-lapse seismic surveys in order to find out what is incorrect in the reservoir model, in a way similar to the production history matching familiar to reservoir engineers as they look for improvements to the model. This being the case, it is difficult to determine in advance of monitoring just what it is we should be monitoring, and survey that are designed specifically to test one feature of a reservoir model may be missing other important features. In this part of the report, we present a set of very surprising results from the Teal South Time-Lapse Multi-Component (4D-4C) study, in Eugene Island Block 354 in the Gulf of Mexico. We will show that time-lapse seismic observations have revealed that an undrilled reservoir near a producing reservoir is exhibiting time-lapse changes consistent with expansion of a free gas phase, and that this implies that oil is being lost through the spill point, never to be recovered, even if that reservoir is eventually drilled for production.

The Teal South 4D-4C study has provided seismic data sets covering three different times: one time prior to production ("legacy" streamer data), and at two times after production (Phase I and 
Phase II, each using 4-component ocean-bottom cables). The project, initiated by Texaco, has been continued through a consortium organized by the Energy Research Clearing House, and some of the results of this project have been described in previous articles in TLE (Ebrom and others, TLE, March 2000; Entralgo and Spitz, TLE, June 2001). Although this project was originally designed specifically as a test of seismic technologies, it has evolved into a test of petrophysics and reservoir models as well.

The field of most interest to the study is the so-called "4500-ft" reservoir (labeled "A" on Figure 98); it quickly generated free gas under production, having been near bubble point at discovery, and appears to have developed a zone of encroached water as well as a gas cap. Reservoirs with an initial liquid expansion drive mechanism undergo an initial very rapid decrease in pore pressure, followed by a less rapid, but nonetheless steady, continued decline in pressure during the solution-gas-drive phase of production (with moderate water drive in this case). The production history of the 4500- $\mathrm{ft}$ reservoir is summarized in Figure 99. Conventional wisdom had predicted that the seismic response of the 4500-ft reservoir would consist of continued 'brightening' of this Class III AVO reservoir with production as the gas saturation continuously increased. A prediction was made (Pennington, 2000) that the situation would actually be quite a bit more complicated: due to the fluid-substitution effects alone, there should be continued brightening at all offsets; but, because there is also an increase in effective confining pressure as a result of pore-pressure decline during production, this bright spot should dim at near offsets after the initial brightening from the exsolution of free gas. This type of petrophysical behavior has also been proposed by Landro (2001) and Bentley et al (2000). Details of our new approach are included later in this report.

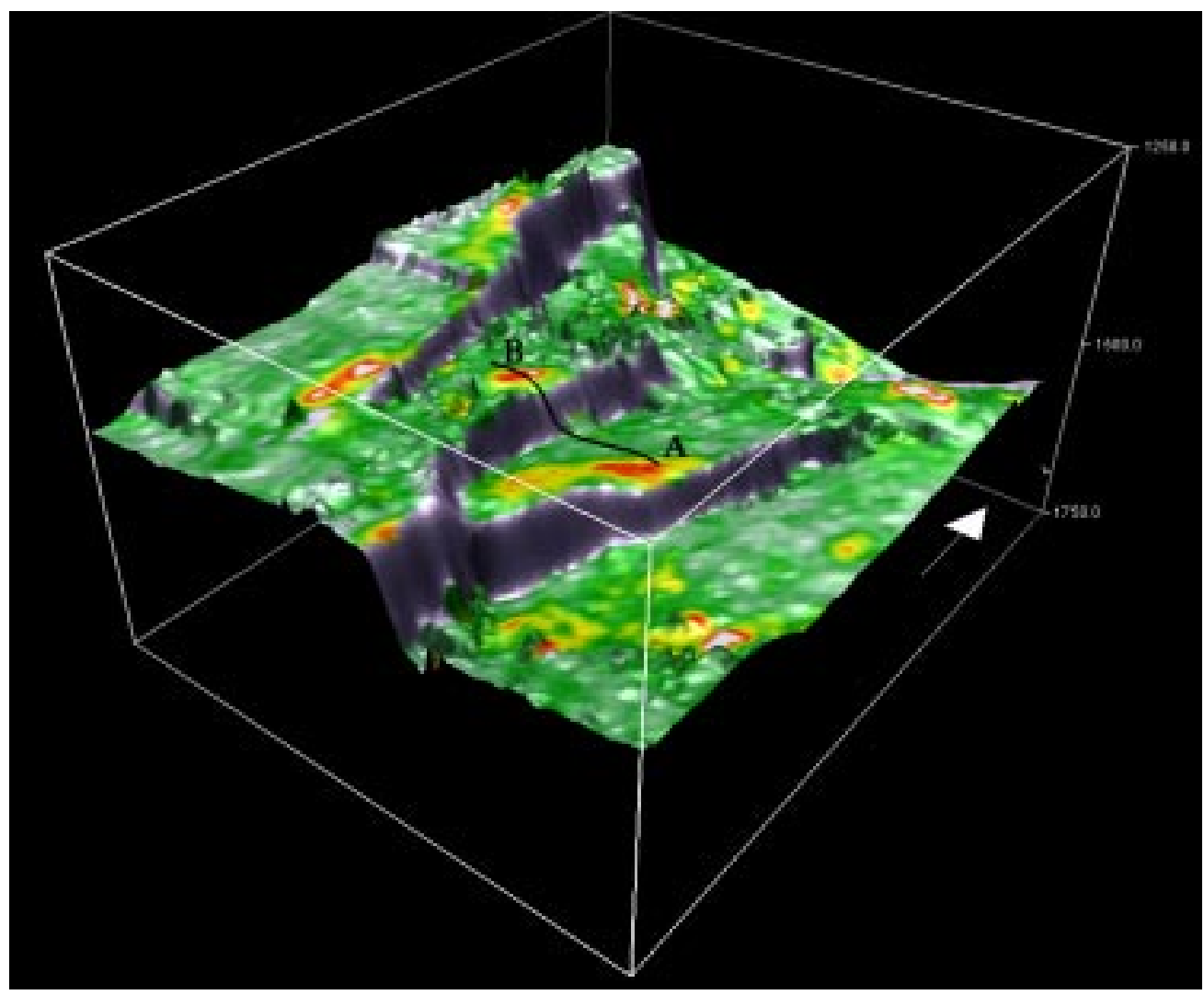

Figure 98: 3D perspective view of the 4500-ft sand.

The currently producing '4500$f t$ ' reservor is labeled A. Nearby undrilled potential reservoirs are visible as bright spots, including the "Little Neighbor" labeled $B$. 


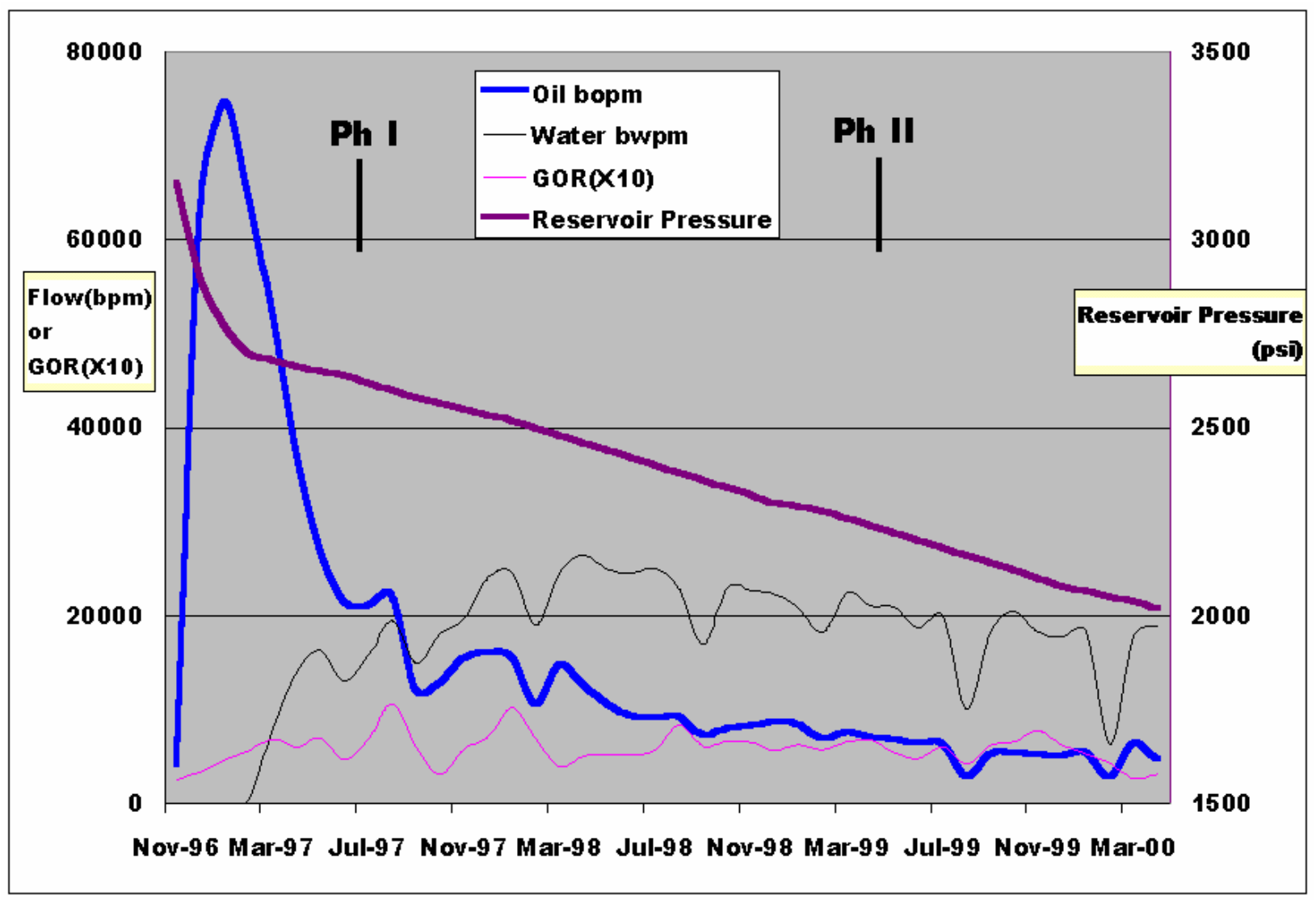

Figure 99: Smoothed production history of the 4500-ft reservoir.

Tthe Oil flow rates (in bbls of oil per month), water flow rates (bbls of water per month), and Gas-Oil-Ratio (GOR multiplied by 10) are all read from the left axis, and the reservoir pressure (in psi) is read from the right axis. The reservoir pressure is simplified from the results of a reservoir simulation conducted by personnel at Heriot-Watt University. Times of the oceanbottom time-lapse surveys Phase I and Phase II are indicated.

\section{ROCK PROPERTIEs Determination}

In order to test and calibrate the model used in time-lapse seismic petrophysical predictions, we first needed to know the properties of the rocks in situ. The log data were inconclusive, and it was necessary to establish confidence in our petrophysical model through inversion of the legacy seismic data for acoustic impedance (see Figure 100). The final result was a model in which fluid-substitution, using Gassmann theory, was consistent between the oil and water legs of the 4500-ft sand - that is, when we used the values obtained for acoustic impedance in the water sand, and made some simple assumptions for dry-frame Poisson's ratio (which, in this case, were not critical), we were able to predict the values for acoustic impedance observed in the oil sands. Thus, we had a set of rock properties on which to base our predictions for seismic response during production, at least to the degree of accuracy required here. 


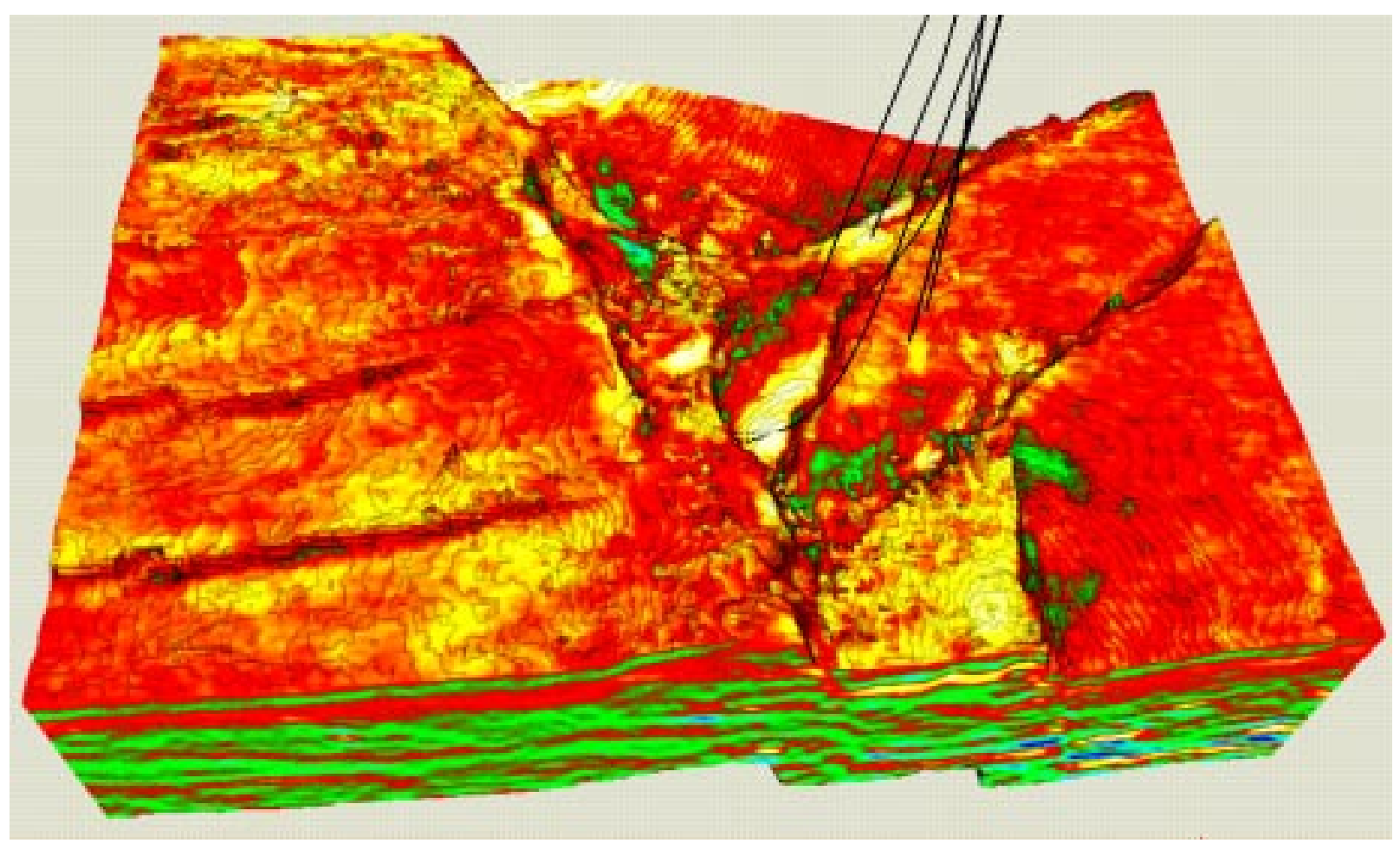

Figure 100: Inverted (legacy data) of the reserovoir interval.

Horizon shown is 12 ms below the top of the tracked 4500-ft horizon, inside the reservoir intervals. The green color indicates high impedances (shales), red colors indicate intermediate impedances (water sands), and the yellow colors indicate low impedances (oil sands).

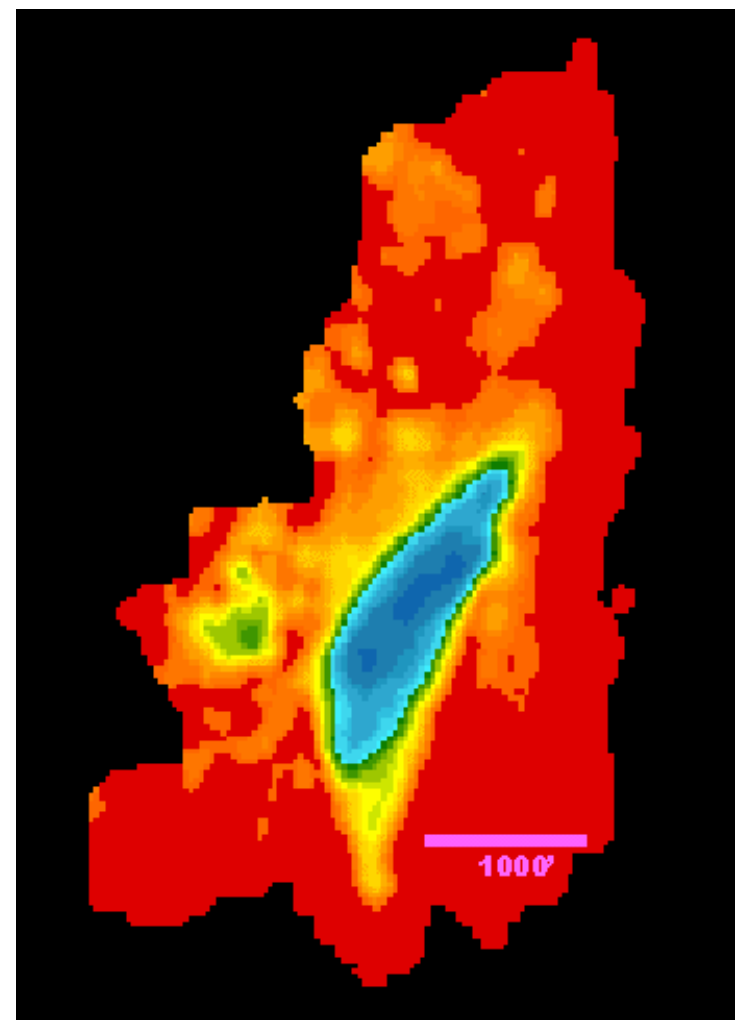

At the same time, additional nearby reservoirs were identified (such as the "Little Neighbor" labeled "B" in Figure 98), and were occasionally of interest to the investigators in the Teal South consortium. The production history from the producing fields was known, yet some effects were consistently showing up in the undrilled reservoirs. For example, Figure 101 shows a difference image obtained by subtracting the amplitudes on the 4500-ft horizon between Phases I and II.

Figure 101: Time-lapse difference.

Mapped on the 4500-ft horizon, showing the change in amplitudes of the stacked seismic data between Phase I and Phase II. The 4500-ft reservoir and the Little Neighbor reservoir both show significant changes (blue and green), although only the larger 4500-ft reservoir is under production. (Image provided by W. Haggard, C. Vuillermoz, S. Spitz, and $P$. Granger of $C G G$,.) 
The model for fluid substitutions due to changing gas saturation and for frame stiffening due to increased confining pressure indicated (Figure 102) that the P-wave velocity should initially decrease and then increase significantly during production, while Poisson's ratio should continually decrease.

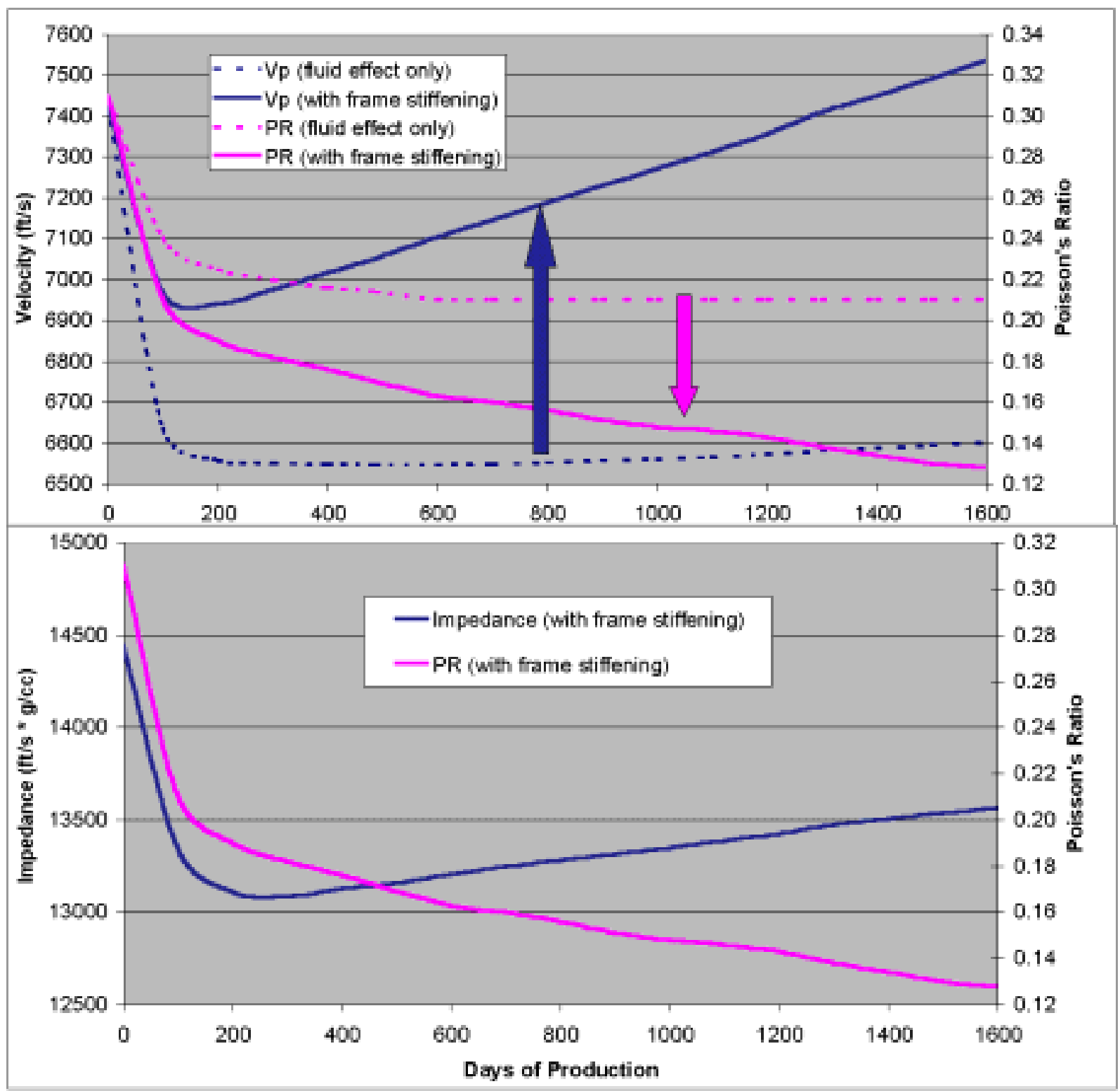

Figure 102: Changes in P-wave velocity (Vp), Poisson's ratio (PR), and Acoustic Impedance with time of production.

The upper graph shows the result of the fluid substitution calculations only (dotted lines) and with the inclusion of frame stiffening effects (solid lines). Notice that the frame stiffening effect eventually more than cancels the reduction in velocity due to the fluid substitution, while enhancing the Poisson's ratio effect. Inclusion of the density changes (oil to gas) decreases the impact of frame stiffening on the impedance results (lower graph), but following an initial dramatic decrease, the impedance increases with time during most of the life of the field. This 
scenario results in an amplitude-versus-offset effect (Figure 103) that includes an initial brightening at all offsets, followed by a dimming at near offsets and a continued brightening at far offsets. Notice that the far offsets are expected to continuously increase in amplitude as production continues, but that the near offsets will initially increase, then subsequently decrease in amplitude. Phase I seismic data was collected about 230 days after production began, and Phase II about 950 days.

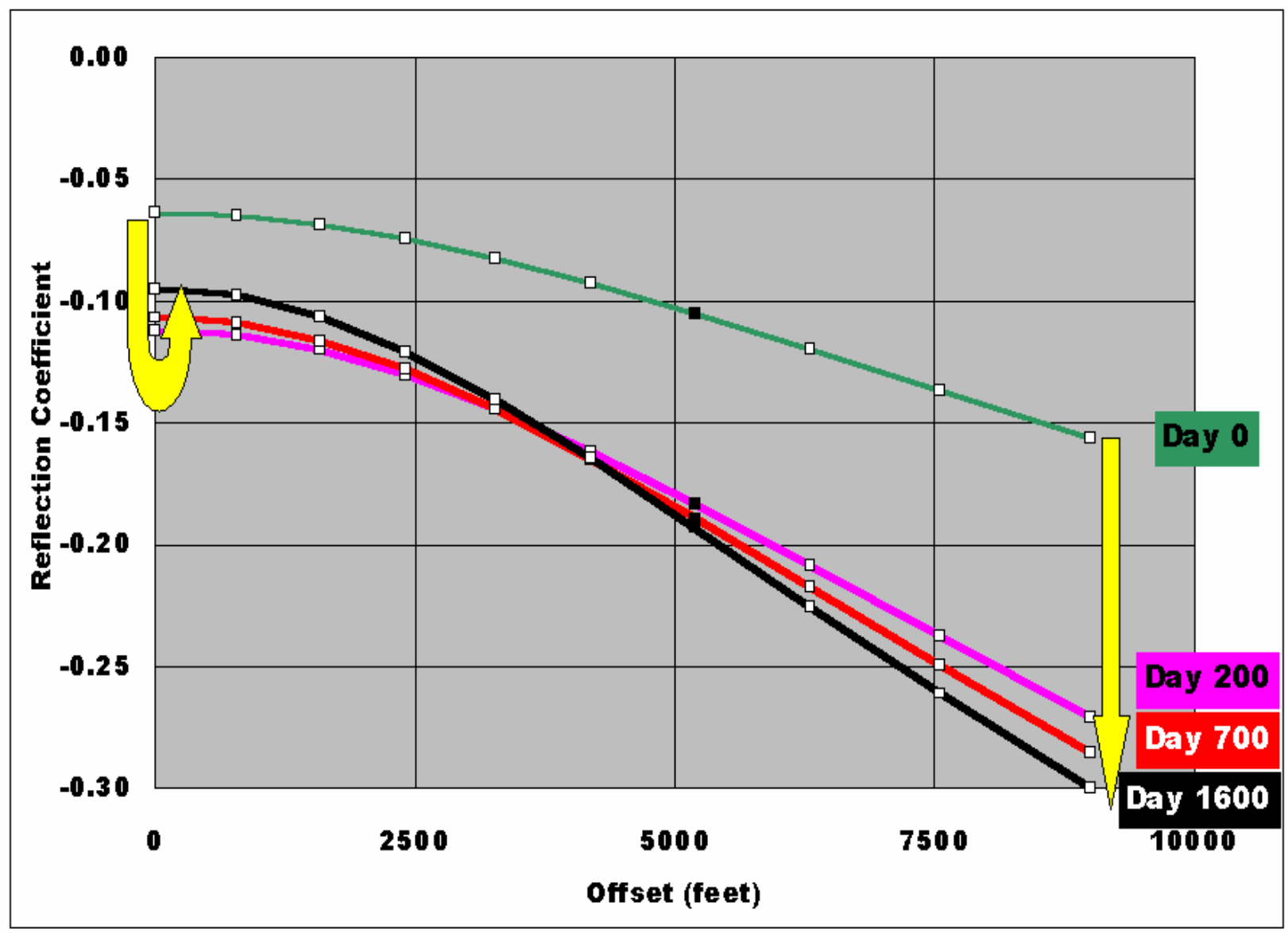

Figure 103: Predictions for Amplitude-versus-Offset effect to be observed in time-lapse data for Teal South. Small squares indicate each additional 5 degrees in angle of incidence, and the black square indicates 30 degrees.

\section{Pre-Stack Time-Lapse Seismic Observations}

We chose to investigate the pre-stack behavior of the reflections from the 4500-ft reservoir and from the Little Neighbor reservoir in the OBC data from Phase I (shortly after the initial release of free gas) and Phase II (after a couple years of continued production). Because the seismic traces are not equally distributed among the offset ranges and their distribution varies among cdp gathers, we grouped the offset traces into different ranges and constructed partial stacks within each range. Results are presented here for every fourth cdp gather along one east-west line intersecting both reservoirs, as indicated in Figure 98. The reflections from the 4500-ft reservoir (Figure 104) show that the far offsets increased in amplitude between Phases I and II, while the small-amplitude near-offsets remained essentially constant. 


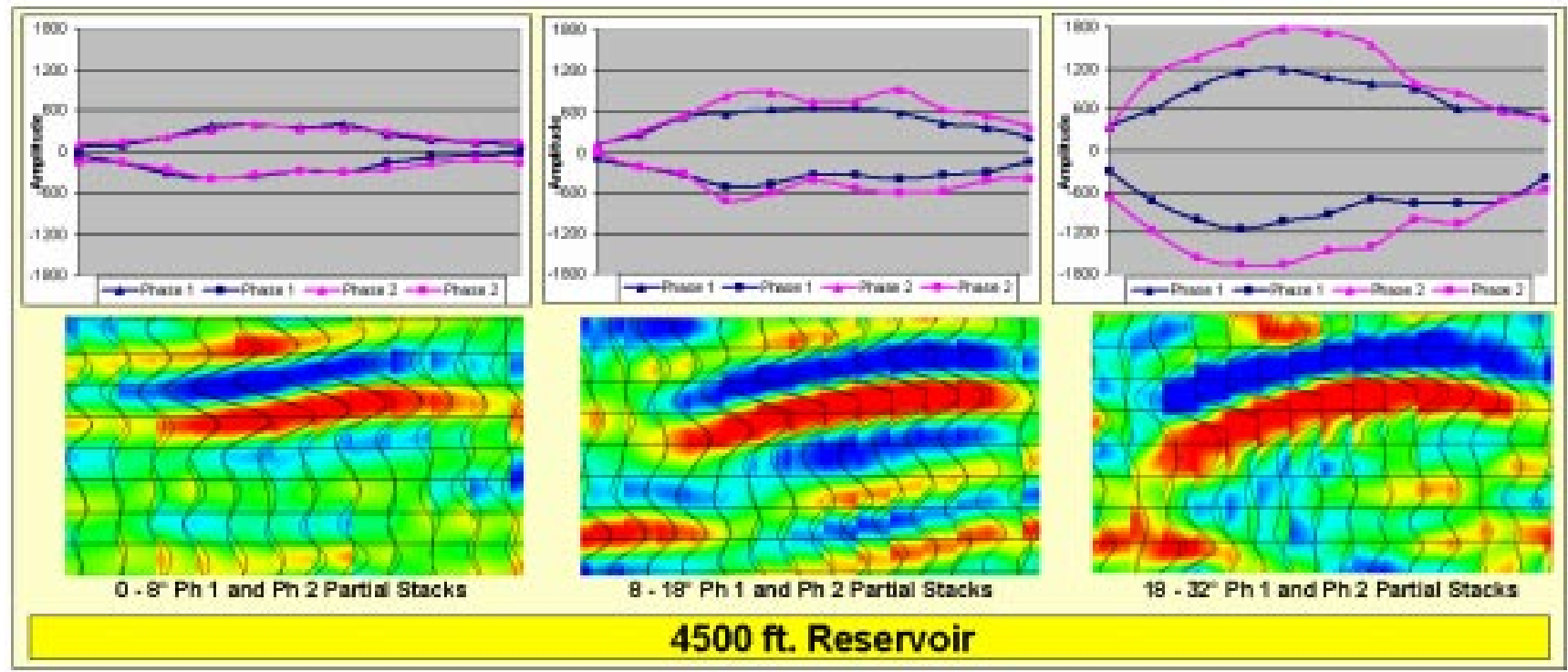

Figure 104: Amplitudes versus offset in the time-lapse data for the producing reservoir. Amplitudes extracted from the partial-offset (unmigrated) stacked seismic P-wave data for the 4500-ft reservoir from Phases I and II. The upper diagrams show the amplitudes of each partialstacked trace, measured on the upper trough and the lower peak as shown on the lower diagrams This figure agrees with the model prediction shown in the previous figure.

The reflections from the "Little Neighbor" reservoir (Figure 105) show that the same situation exists, except that the near-offsets actually decreased slightly in amplitude between the two phases. Both reservoirs show characteristics (within noise limits) of reservoirs that have released free gas, and which continue to increase gas saturation while decreasing reservoir pore pressure.

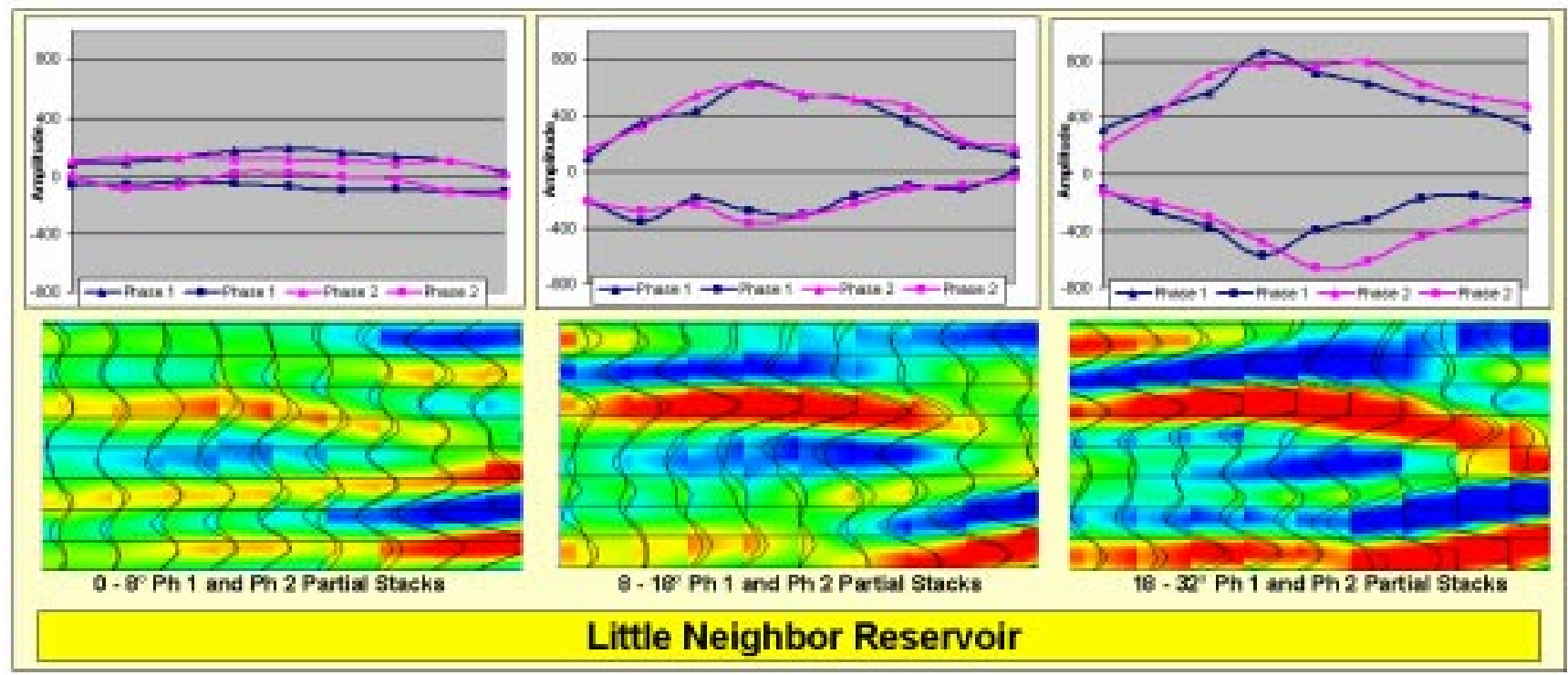

Figure 105: Amplitudes versus offset for the nearby unproduced reservoirs.

Amplitudes extracted from the partial-offset stacked seismic P-wave data over the Little Neighbor reservoir. Compare with the observations for the 4500-ft reservoir in the previous figure, even though this reservoir is not being produced. 


\section{INTERPRETATION}

This behavior contains two surprises for the conventional viewpoint: First, the amplitudes do not monotonically brighten as additional gas is released; instead, the near-offsets eventually decrease (from one time-lapse survey to another after an initial brightening), while the far-offsets increase in amplitude. Second, the Little Neighbor reservoir, originally thought to be separated by sealing faults, is responding to production in the 4500-ft reservoir in a manner that is remarkably similar to the seismic response exhibited by that reservoir. Our conclusion is that the Little Neighbor reservoir is undergoing a decline in pressure due to the production of the $4500-\mathrm{ft}$ reservoir. It must be in pressure communication through some route within the formations for this to occur, and therefore not isolated by the faults which bound either reservoir. By examination of the inverted acoustic impedance volume along an arbitrary seismic path that links the downdip ends of each reservoir, we find that there is indeed a path of continuous (water) sands that connect the two reservoirs, and perhaps others as well (Figure 106).

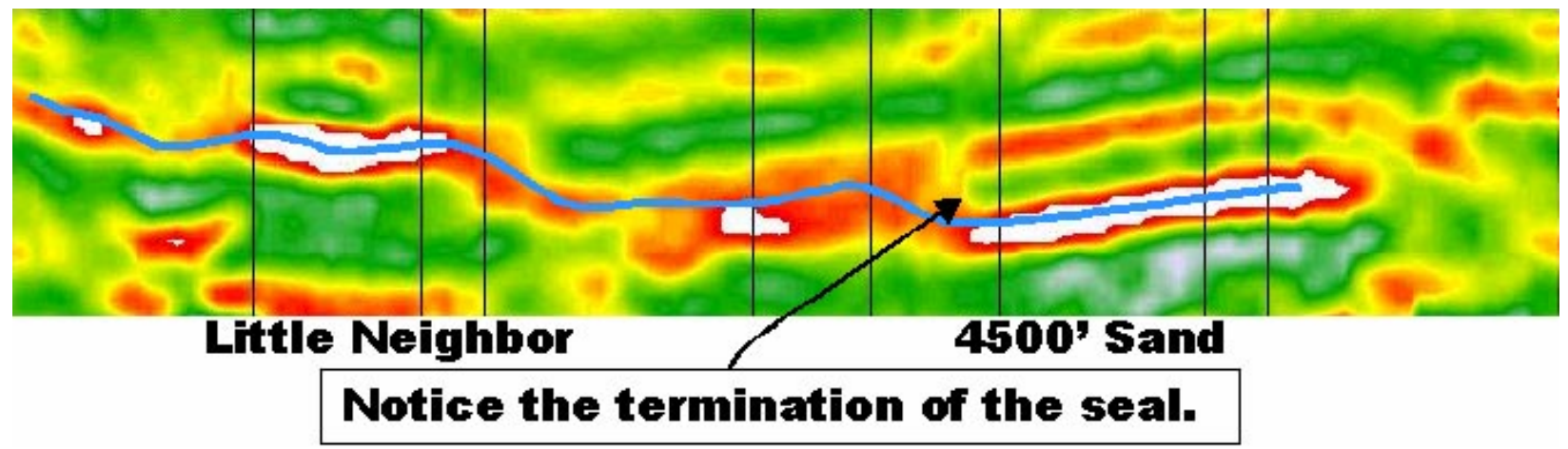

Figure 106: Traverse through the inverted acoustic impedance volume from the legacy data set. Connecting the downdip ends of the 4500-ft reservoir and the Little Neighbor reservoir, the ends of the section meet, providing a full circle starting (at the left edge) updip of the Little Neighbor reservoir, with bends in the section at each vertical line, extending, clockwise, through the 4500ft reservoir, finally returning to the starting point. White is used to highlight the lowestimpedance sands (those likely containing light hydrocarbons); red indicates impedance corresponding to water sands; and green indicates impedance corresponding to shales. Note that the shales form traps, but the each reservoir has its own spill point into water sands; the reservoirs are connected, and in pressure communication, through these spill points.

There are serious implications for reservoir management contained in this interpretation. The fact that the Little Neighbor appears to be in pressure communication and that it exhibits a seismic response appropriate for the creation of a free gas phase results in a volume accommodation problem. The free gas occupies more volume than the oil from which it was released; usually, this volume is more than accounted for by the production of the oil. But in the Little Neighbor's case, the oil contained within it is not being produced. It must be moving down-structure within the formation as the gas cap grows. But downstructure there is no trap to contain it - there is only the spill point (see Figure 107 for a cartoon version of this scenario). The displaced oil of the Little Neighbor reservoir can not migrate to the 4500-ft reservoir and be produced there - that is much too far downstructure, and there are many routes for the oil to escape prior to reaching it. Instead, the oil is likely escaping through the spill point, and either pooling in some other local trap, or escaping into the overlying sands. This oil is likely to be lost for ever, inaccessible to future production, unless it happens to be trapped in some upper zone 
with economics favorable for recovery. If a well were to be drilled into the Little Neighbor reservoir at this time, the oil in place would be found to be much less than that estimated from the legacy data, obtained prior to production of the nearby $4500-\mathrm{ft}$ reservoir.
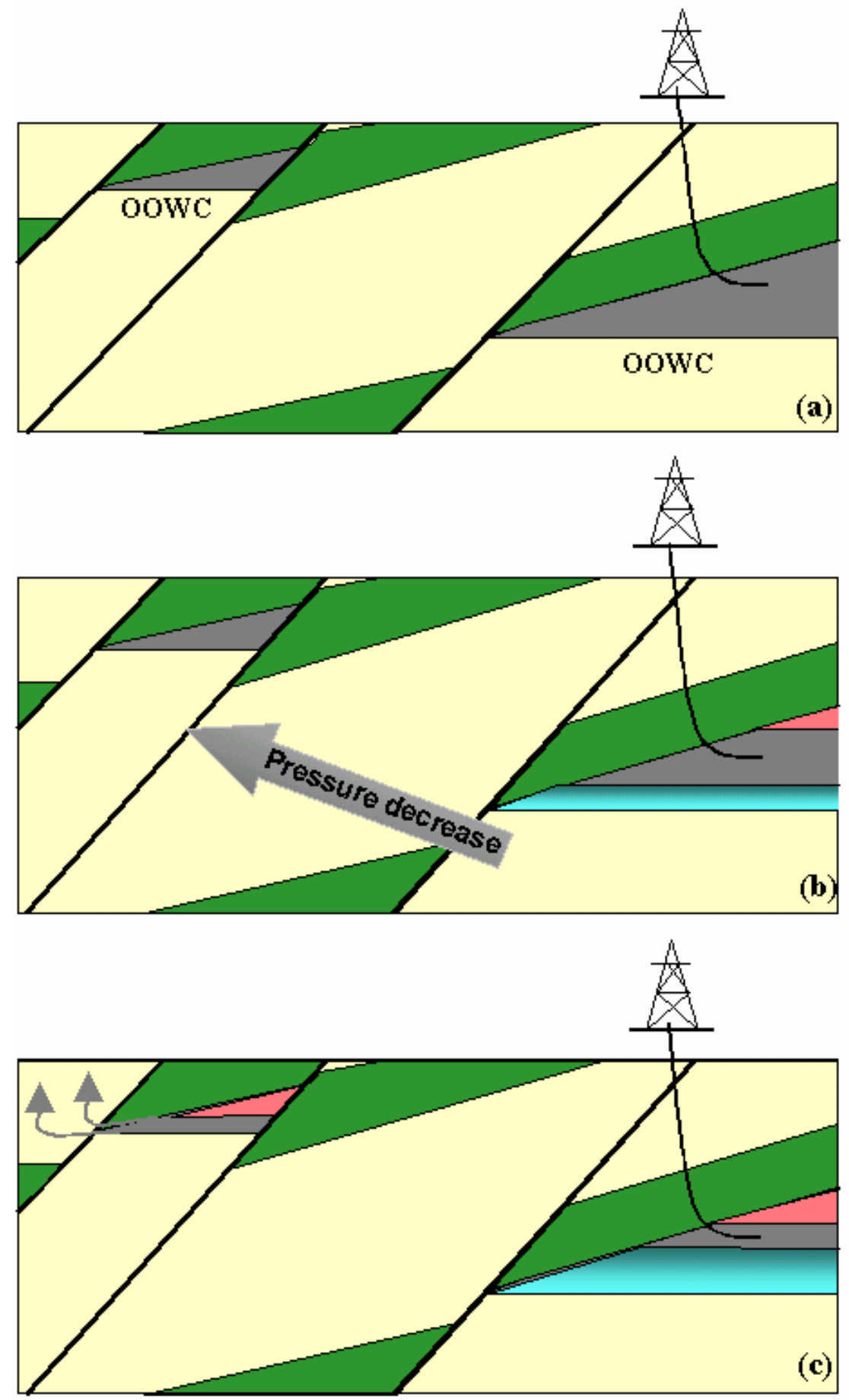

Final Technical Report October, 2002
Figure 107: Cartoon schematic of the sequence of events resulting in the loss of hydrocarbons from the Little Neighbor due to production in the $4500-f t$ reservoir.

The 4500-ft reservoir is shown to the right, and the little reservoir is shown to the left, in a geometry similar to that shown along the traverse line of Figure 9. OOWC is the Original Oil Water Contact. In (a), production has just begun in the 4500-ft reservoir, and no displacement has yet been observed. In (b), production from the 4500-ft reservoir has resulted in free gas and water encroachment in that reservoir, as well as a pressure decrease that is communicated through the water sands. In (c), that pressure drop has resulted in the creation of free gas in the Little Neighbor reservoir, and the downward displacement of the remaining oil. That oil eventually spills out of the reservoir, likely never to be recovered. 


\section{IMPLICATIONS}

The time-lapse survey of Teal South has yielded some completely unexpected results that could be of significant importance for reservoir management of the fields in this block. For this reason, it may not be advisable to design time-lapse surveys to test only one single aspect of production - we feel that our knowledge of the greater reservoir system is, in general, fairly incomplete, and seismic surveys for time-lapse purposes should be designed to allow for the observation of the unexpected. The Teal South experiment was designed in a manner that permitted us to draw the conclusions presented in this paper, which was fortunate. We do not presently know whether the phenomenon observed here - that production in one reservoir is apparently resulting in the loss of hydrocarbons from another unproduced reservoir - are likely to be widespread in the Gulf of Mexico and elsewhere, or not. But we do know that without the time-lapse seismic observations, we would not have recognized it in this instance. The fully-reprocessed seismic data was used to create a time-lapse difference volume, and the amplitudes along the surface of the 4500-ft reservoir sand are shown in Figure 108. The relative brightness of the events corresponding to the neighboring reservoirs appear to show the path of pressure migration.

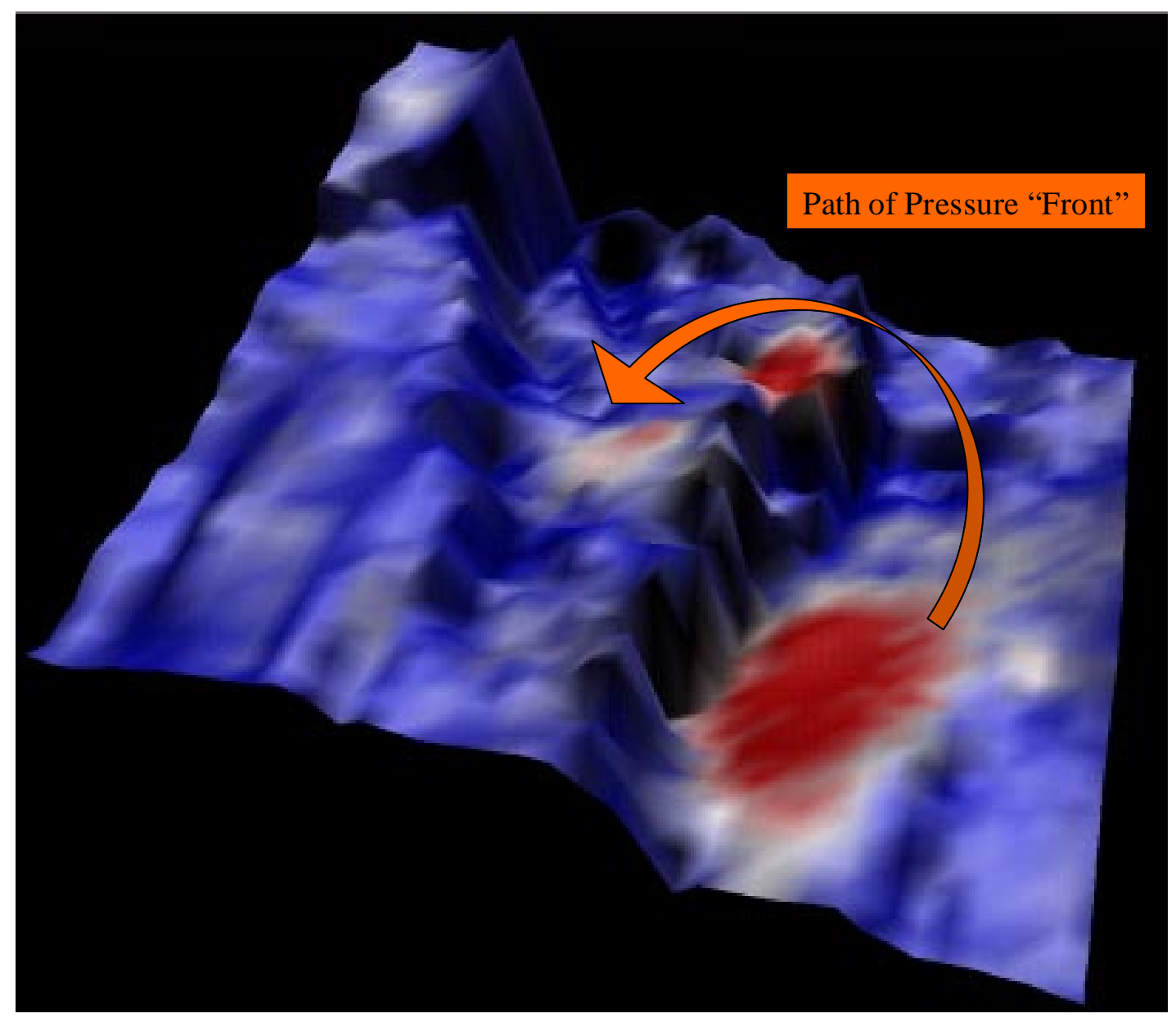

Figure 108: Time-lapse difference image of the 4500-ft sand, showing the relative brightness of the nearby reservoirs and the apparent path of pressure migration. 


\section{EFFECTIVE PRESSURE CHANGES AND SEISMIC VELOCITIES}

\section{Background}

As any reservoir is produced, changes in subsurface rock and fluid properties will occur. This study focuses particularly on how seismic velocities of the rock matrix can change due to pressure changes that often accompany production.

Three types of pressure are of interest in this study: Overburden pressure (confining pressure), pore pressure (reservoir pressure), and effective pressure (here assumed to be identical to differential pressure). Effective pressure $\left(\mathrm{P}_{\mathrm{e}}\right)$ is defined as this difference between confining pressure $\left(P_{c}\right)$ and pore pressure $\left(P_{p}\right)$, such that, $P_{e}=P_{c}-P_{p}($ Wyllie 1962). This expression is not as useful at high pore pressures and confining pressures, where $\mathrm{P}_{c}$ and $\mathrm{P}_{\mathrm{p}}$ do not cancel each other out (Prasad and Manghnani 1997), and a distinction between differential pressure and effective pressure should be made. As a reservoir is produced, effective pressure increases, due to a decrease in pore pressure (Craft et al., 1991).

\section{Micro-crack closure}

The most important pressure effect on velocity is due to closing of pores as effective pressure increases. Velocity-pressure curves are characterized by an increase in velocity that is much sharper at lower effective pressures (Figure 109). The steeper slope in this region is due to the closure of the microcracks. The velocity increase at higher effective pressures is much lower, and nearly linear, since all the thin cracks have closed.

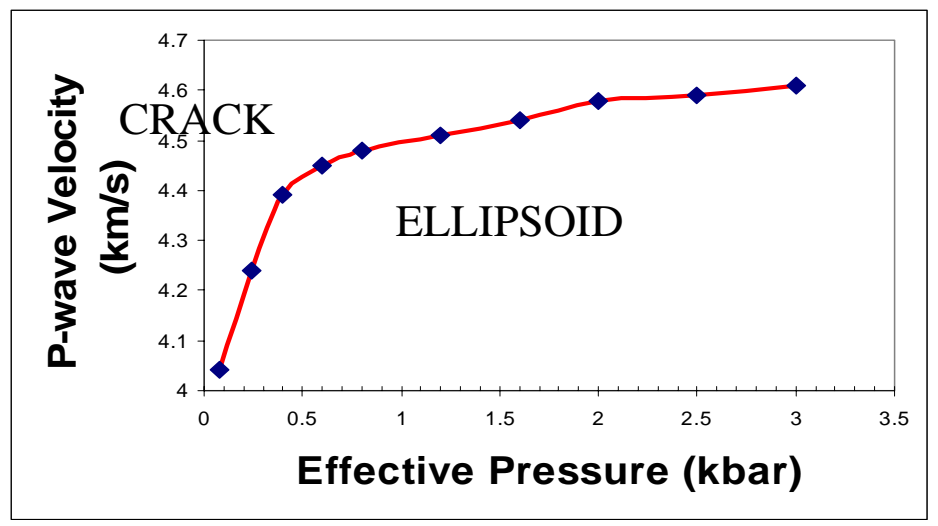

Figure 109: Sharp velocity increases are shown on the left of the graph, due to cracks closing. The velocity increase to the right is very gradual, as the remaining ellipsoids slowly close with pressure.

\section{Velocity-Pressure Data}

The aim of this paper is to develop an empirical relation to predict velocity changes (and therefore bulk modulus changes, also) as effective pressure changes in the microcrack closure region. In order to develop these relations, one data set was used, based on 67 samples studied by De-Hua Han (Han, personal communication). A second data set of 88 samples studied by Freund (1992) was used to test the general applicability of the relations. 
The velocities had been measured on dry samples at ultrasonic frequencies using the pulse transmission technique. For a discussion of the pulse transmission technique see King, 1966. For the Han data set, the dry samples were also saturated and velocity measurements were taken on these wet samples; these saturated velocities were published in Han et al., 1986, but the drysample velocities have not been previously published. The error in P-wave velocities was about 2 percent and the error in S-wave velocities was about 3 percent. Further details concerning these data sets can be found in Freund, 1992 and Han et al., 1986.

\section{Dry Rock vs. Wet Rock}

Most velocity-pressure data is obtained from tests in the laboratory on wet (or saturated) rocks. At first, this may seem to make sense, since any rock in the ground is going to be more saturated than dry. However, rocks under the ground are measured by low frequency seismic pulses, while rocks in the laboratory are measured with a high frequency sonic pulse. The difference turns out to be quite important. Low frequency sonic pulses allow time for the fluids to 'squirt' out of the thin cracks into the ellipsoidal cracks as the pulse compresses the more-compliant cracks of the rock. With low frequency pulses there is enough time in between each pulse for the fluid to flow and equilibrate. High frequency sonic pulses are much more rapid, so the fluid within the thin cracks never has the chance to squirt all the way out into the stiffer equant pore spaces. The fluid thus remains in the thin cracks, and this provides an additional incompressibility, or stiffness, giving an overestimate of bulk modulus (which also causes an overestimate in velocity). Laboratory measurements done on saturated rocks will therefore give overestimates of velocity at seismic frequencies. It can be more accurate to perform laboratory measurements on dry rock, and then convert the dry modulus (or velocity) into wet modulus (or velocity) using Gassmann's equation (Murphy et al, 1986).

\section{Equation Description}

In order to develop an empirical equation that relates effective pressures to compressional and shear wave velocities (or to changes in velocities), previous works in this area were studied. Of particular interest were empirical equations published by Eberhart-Phillips et al, 1989 and Bentley et al, 2000.

Eberhart-Phillips attempted to predict velocity from effective pressure using an exponential relationship:

$$
V=A+K P_{e}-B e^{\left(-D P_{e}\right)} \quad \text { (eq.1) }
$$

The physical meaning behind these coefficients is as follows: A is the crack-free velocity, effected by porosity and clay content, $\mathrm{K}$ is the linear section slope on the velocity-stress curve, $\mathrm{D}$ relates to the closure of microcracks, and A-B indicates velocity at zero effective stress (Khaksar et al., 1999).

Using saturated rock samples that included $\mathrm{P}$-wave and $\mathrm{S}$-wave velocities at pressure ranges from .05 kbar to .50 kbar (Han 1986), Eberhart-Phillips found best fit values for coefficients A, $\mathrm{B}, \mathrm{D}$, and $\mathrm{K}$ for every rock sample in the Han data set. They attempted to correlate the best-fit values of these coefficients to measured values of clay content or porosity. They found that the 
pressure dependence based on these coefficients did not have a statistically significant relationship with clay content or porosity.

Bentley developed equations to predict changes in dry bulk modulus, $\mathrm{K}_{\mathrm{D}}$, and dry shear modulus, $\mu_{\mathrm{D}}$ (from which changes in P-wave and S-wave velocities are easily derived):

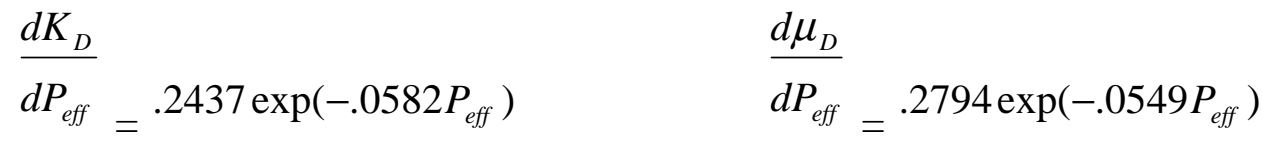

(These expressions assume pressure is measured in MPa, and changes in bulk modulus are also given in MPa. The key to using Bentley's equations is the assumption that bulk modulus and shear modulus (and therefore seismic velocities) are known at one pressure.

This assumption can be utilized in other equations, too, such as the Duffy-Mindlin relationship (White, 1983; Merkel et al., 2001). The assumption is realistic, since in most fields where time lapse seismic is of interest, well logs contain velocities known at the original, pre-production pressure. This assumption, then, adds another "known" to equations that originally were developed to predict velocities using pressure, clay content and porosity alone. Figure 110 shows how much more accurate velocity predictions can be if a seismic velocity is known at just one pressure.

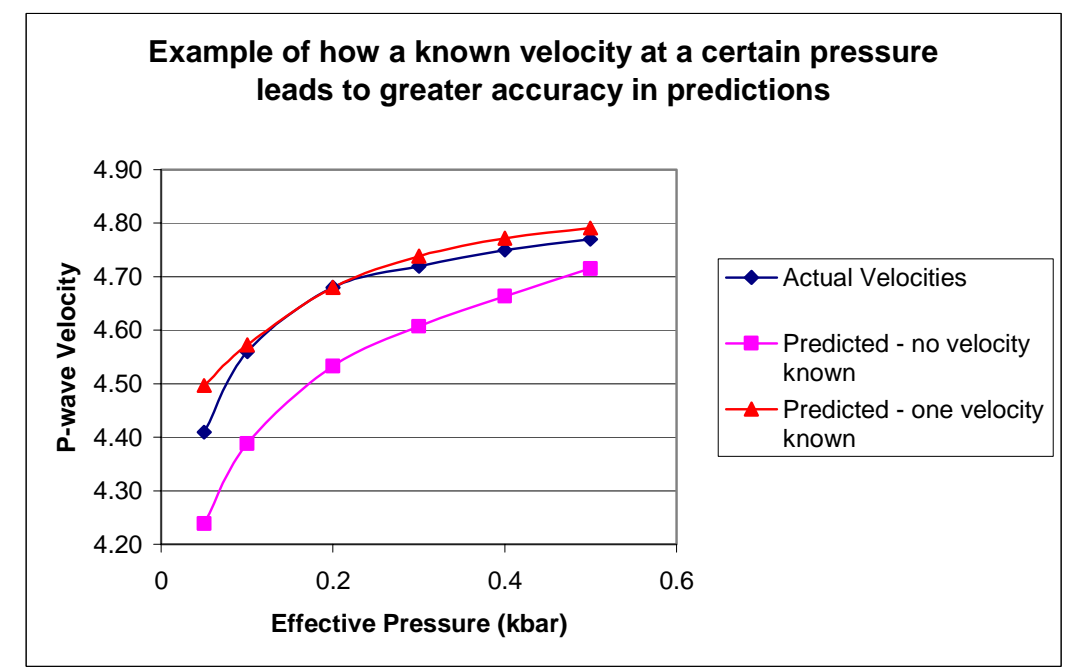

pressure and velocity.
Figure 110: Velocity predictions tied at a point are more accurate than general velocity curves

This is a significant difference, since in other work on this relationship, best fit values for coefficients A, B, K, and D were estimated assuming these coefficients were a function of pressure alone. In this study, since we assume we know a velocity at a certain pressure, we can solve for the best fit coefficients as a function of

It was recognized that if we know a velocity at one pressure, then that adds one more parameter (besides clay content and porosity) to which the coefficients A, B, D, and K could be related. This assumption that we have a known velocity is one of the mainpoints that distinguish the empirical equation formed in this study or Bentley's from others. The further distinguishing factor is our use of dry (unsaturated) rock data instead of wet (saturated) rock data; Bentley's equations were developed using the data published by Han et al., 1986, which were provided for saturated rock samples only. 


\section{Development of the equation}

Eberhart-Phillips' original equation was:

$$
V=A+K P_{e}-B e^{\left(-D P_{e}\right)}
$$

We simplified this equation by combining coefficients $\mathrm{B}$ and $\mathrm{K}$, so that

$$
V=A+B P_{e}-B e^{\left(-D P_{e}\right)}
$$

- or -

$$
V=A+B\left(P_{e}-e^{\left(-D P_{e}\right)}\right)
$$

(As a side note, many others have simplified Eberhart-Phillips' to $\mathrm{V}=\mathrm{A}-\mathrm{Be}^{(-\mathrm{DPe})} \quad$ similar to our equation, except without the $B \times P_{e}$ term). This equation is of the form $y=m x+b$, where $\mathrm{y}=\mathrm{V}, \mathrm{m}=\mathrm{B}, \mathrm{b}=\mathrm{A}$, and $\mathrm{x}=\left[\mathrm{P}_{\mathrm{e}}-\mathrm{e}^{(-\mathrm{DPe})}\right]$ (also termed $\mathrm{P}$ '). For every rock sample, we plotted dry $\mathrm{P}-$ wave velocity vs. $\left[\mathrm{P}_{\mathrm{e}}-\mathrm{e}^{(-\mathrm{DPe})}\right]$ (Figure 111$)$. (Actually, we plotted velocity vs. $\left[\mathrm{P}_{\mathrm{e}}-\mathrm{e}^{(-\mathrm{DPe})}\right]+1$, since it was recognized that at certain values of $\mathrm{D}$ and $\mathrm{P}_{\mathrm{e}}$, the $\mathrm{P}$ ' term would equal zero, and therefore could cause divide-by-zero problems). In this analysis, the velocities measured at the lowest pressure (.05 kbar) were excluded for reasons that will become evident later. Coefficient $\mathrm{D}$ was varied until the best linear correlation between velocity and $\mathrm{P}$ ' +1 was found. The slope for this best fit value would be coefficient $B$, and the intercept at the best fit value would be coefficient A.

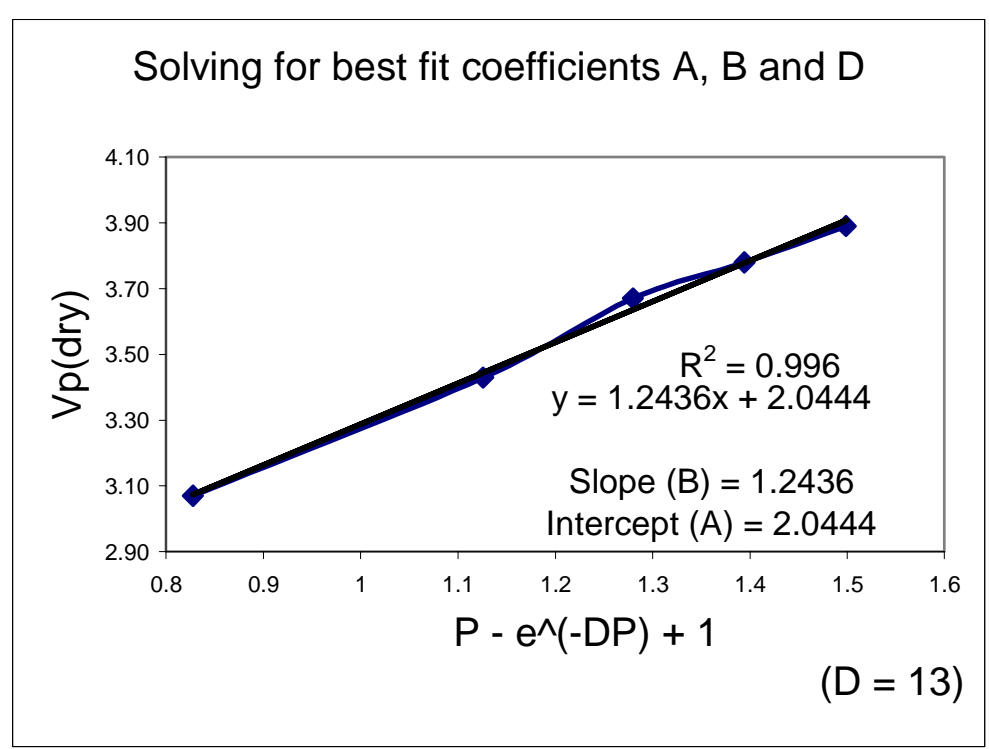

Figure 111: Fitting $P_{e}-e^{-D P e}+1$ to velocities to solve for coefficient $A$ and $B$. Coefficient $D$ was varied until the least $R^{2}$ value was found.

In this way, best fit values for coefficients A, B, and D were found for every rock in the data set of dry P-wave velocity. This method was repeated for dry Swave velocity data.

Just as Eberhart-Phillips tried to correlate clay content and porosity with their coefficients found using Han's wet rock data, we tried to correlate clay content, porosity, and velocity with our coefficients found using Han's dry rock data. In accordance with EberhartPhillips' conclusion, we found that there was no significant pressure dependent relationship between any of the coefficients and clay content or porosity (Figure 112). This was true for both $\mathrm{P}$-wave and S-wave velocities.

We continued the comparison, to observe if velocity and any of the coefficients have a relation. There was no correlation between velocity and coefficient D (Figure 113). This figure shows no 
correlation between coefficient D and P-wave velocity at $.20 \mathrm{kbar}$, but the lack of correlation is true for both $\mathrm{P}$-wave and S-wave velocities at any pressure. This means that in any empirical equations, an average D value could be used for the entire data set. Although this is not entirely desirable, coefficient D does not affect our equation enough to cause significant errors in predictions.

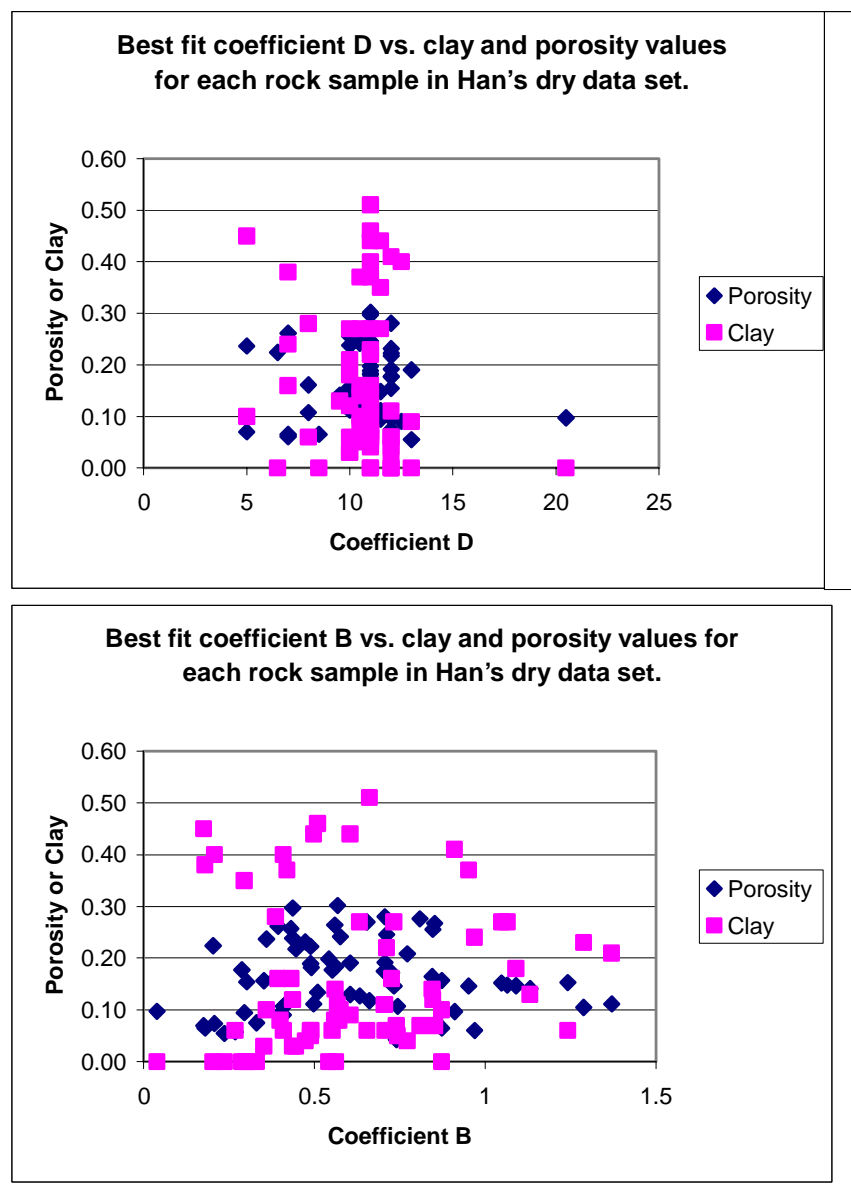

Best fit coefficient A vs. clay and porosity values for each rock sample in Han's dry data set.
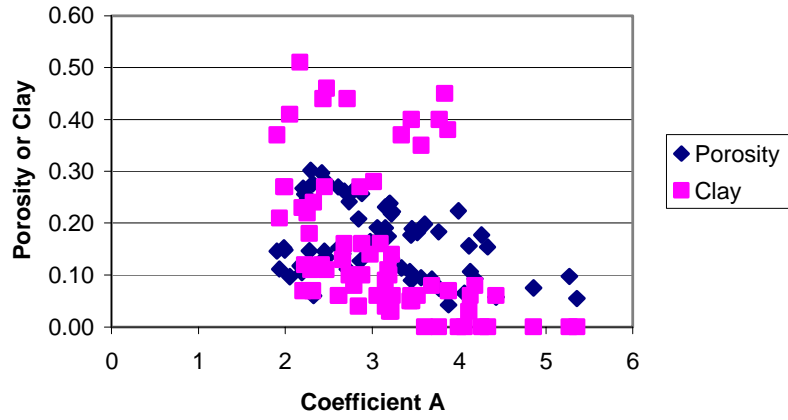

Figure 112: These plots show that there is no correlation between clay or porosity and coefficient A, B or D. These are the same results that were found by Eberhart-Phillips.

Next, having fixed the value of $\mathrm{D}$ at 10.66 , the relation between coefficient $A$ and velocity was examined (Figure 114). Not only are coefficient $\mathrm{A}$ and velocity related at $.20 \mathrm{kbar}$ as shown in this figure, but coefficient A and velocity (either P-wave or

S-wave) show a strong correlation at any pressure. Coefficient $\mathrm{B}$ and velocity relations were also examined (Figure 115). This is not a statistically significant correlation. However, coefficient B vs. change in velocity was plotted, and this does show a strong correlation (Figure 116). The correlation coefficient for velocity changes between pressures of .10 and .20 kbar is

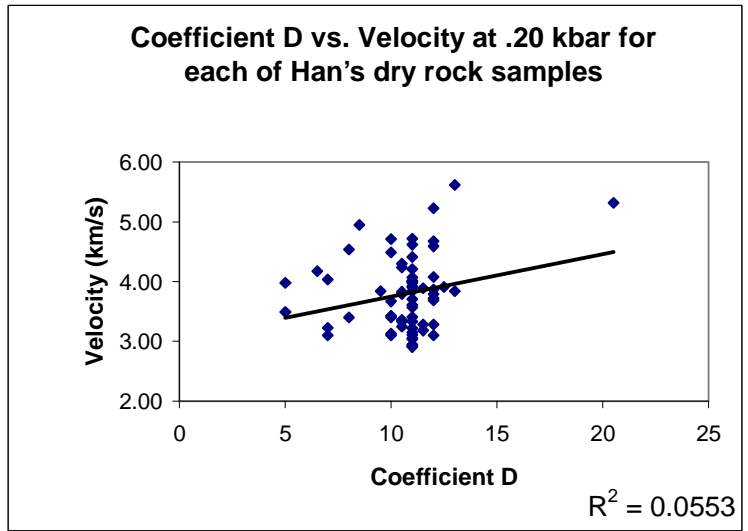
nearly 98 percent for this figure. This correlation coefficient drops as velocity changes between higher pressures are considered, down to about 56 percent for velocity changes between .40 and .50 kbar. However, this shows that there is still some sort of correlation between coefficient B and change in velocity, even at higher pressures.

Figure 113: Best fit coefficient D values show no relation to velocity measurements in Han's data set 

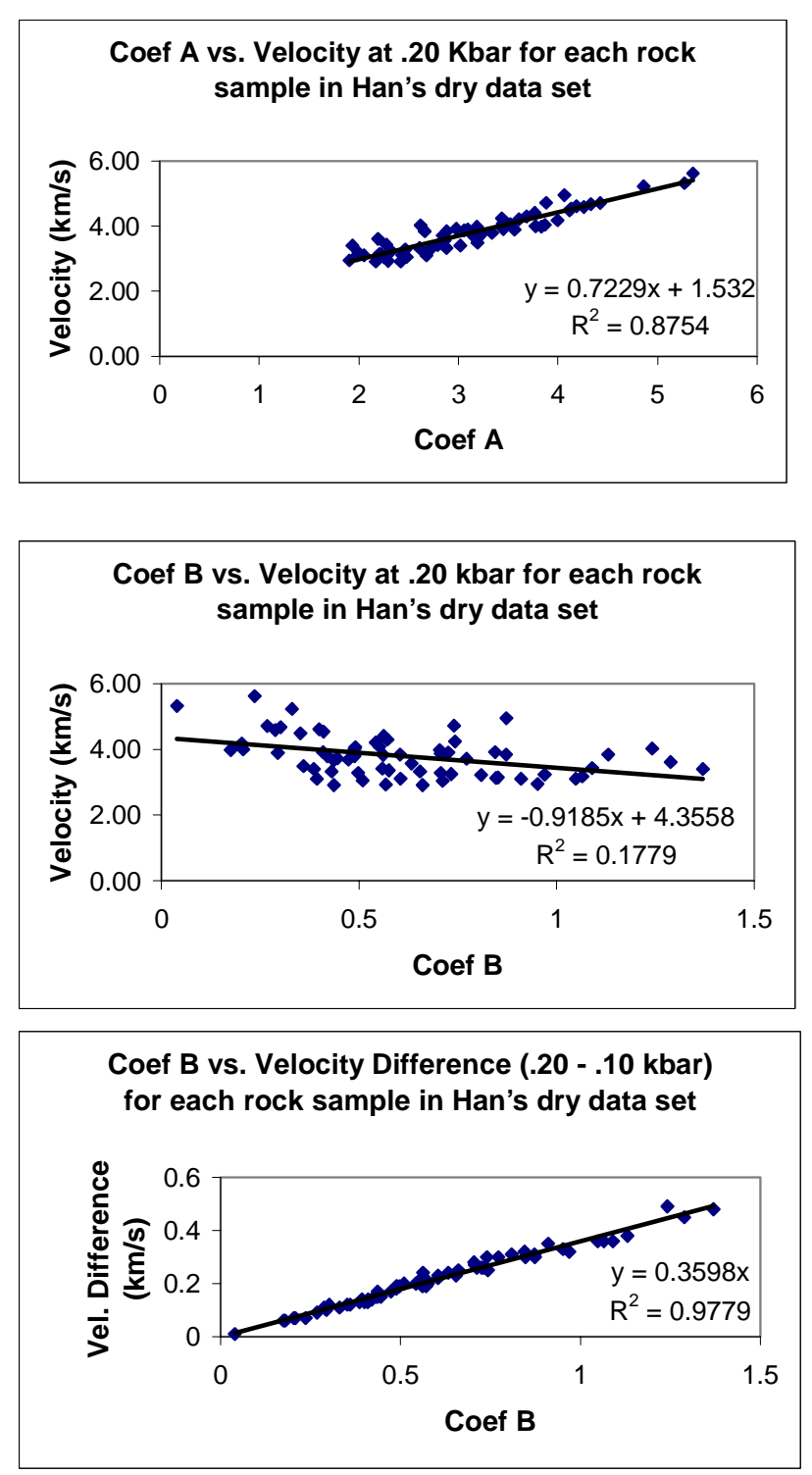

Figure 114 showed that coefficient A can be predicted with a known velocity. This is true at every pressure. But the equation of the line used to make this prediction is different at every pressure, since there are different slopes and intercepts for each of the measured pressures. If we

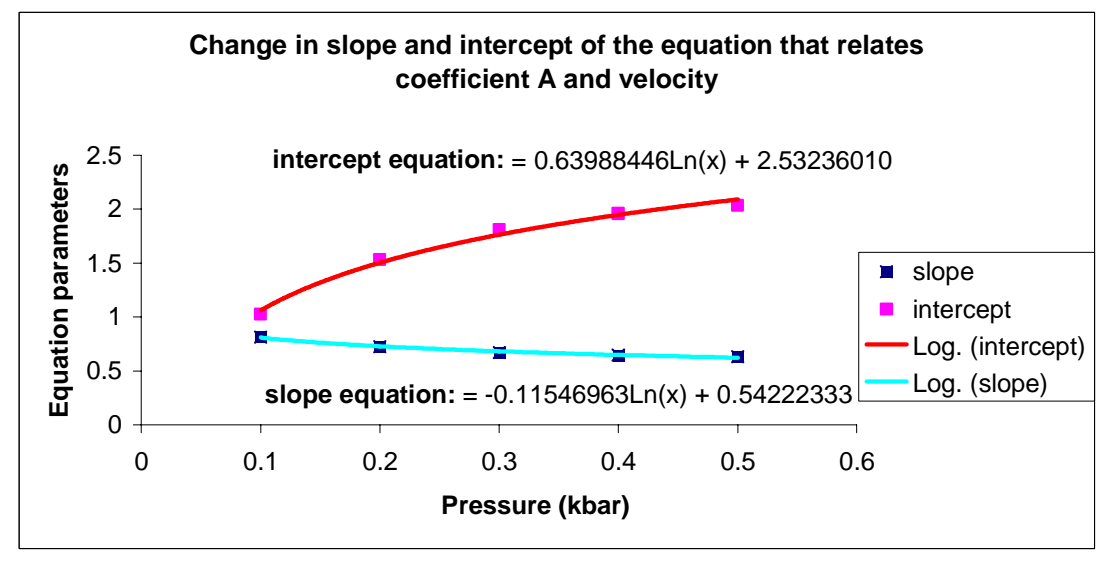

plot these slopes and intercepts against the varying pressure, we obtain a smooth line (Figure 117).

Figure 117: Slope and intercept in the coefficient $A$ vs. velocity best fit line. 
Finding the equation of this line allows us to predict, at any pressure, the slope or intercept that should be used to find coefficient A from a known velocity. For example, if we know the Pwave velocity at a certain pressure - say $3.5 \mathrm{~km} / \mathrm{s}$ at $.23 \mathrm{kbar}$ - we can solve for coefficient A using Figure 117. Just plug in $.23 \mathrm{kbar}$ to find the slope and intercept values - a slope of .7119 and an intercept of 1.592 in this case. Then, we have $\mathrm{V}=.7119 *$ Coef. $\mathrm{A}+1.592$, and since $\mathrm{V}=3.5$, we can solve the equation for coefficient A: 2.68 .

Using the same method to plot the different slopes for the relationship between coefficient B and the change in velocity, a similar plot can be generated (Figure 118).

This equation only gives what coefficient B would be if .10 kbar pressure increments are desired (as is the case in Han's data set). In order to be able to apply this equation between any two pressures, the plot in Figure 118 is integrated, resulting in Figure 119.

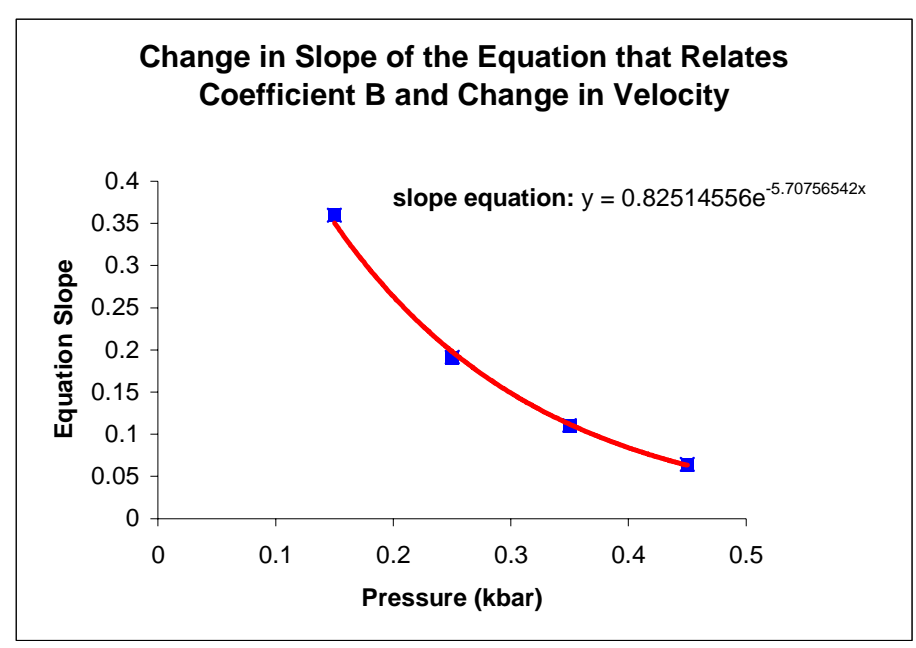

Figure 118: Plot of Table 2, giving the equation that will predict the slope in the best fit line that relates coefficient $B$ and pressure change. Note that the data points for changes between two pressures are plotted at the midpoint of these two pressures.

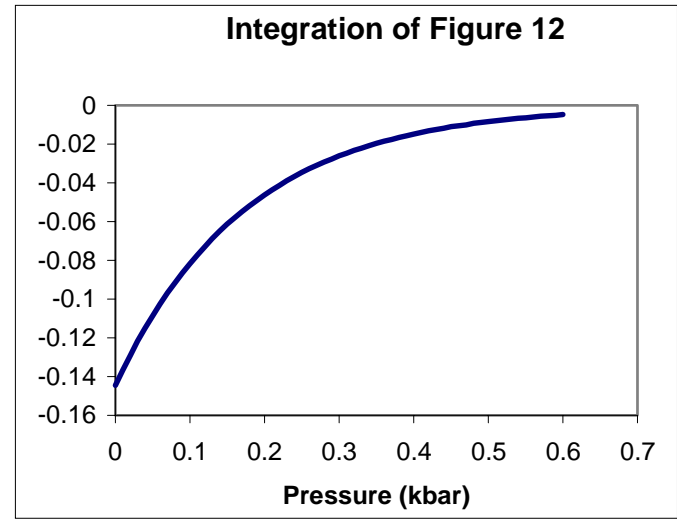

Figure 119: This is simply an example of integrating the equation in figure 11 above, and applying it at different pressures.

The equation of this curve is:

$$
y=-.14457050 \times e^{(-5.70756542 x)} \quad \text { (eq. 5) }
$$

Using this, coefficient $\mathrm{B}$ can be found between any two pressures, by finding $\mathrm{y}$ at a certain pressure, $\mathrm{x}$, and subtracting from the $y$ value at another pressure, $x$. Dividing this by .1 will give the slope, or, in other words, the rate of change of the velocity (in $\mathrm{km} / \mathrm{s}$ ) between two pressures (in kbar). These plots can similarly be generated for $\mathrm{S}$-wave velocities.

The end result of all these correlations, along with the assumption that the equation $1 \mathrm{c}$ is correct, is that an empirical relationship between change in velocity and change in pressure can be developed. A brief summary follows describing the use of these relationships: 
Start with your known velocity and pressure. Use Figure 117 to find the slope and intercept at the pressure of interest. Using these slopes and intercepts, and the known velocity, you solve for coefficient $\mathrm{A}$.

Our original equation was $V=A+B\left(P_{e}-e^{\left(-D P_{e}\right)}\right)$. We have our known velocity at an effective pressure, and we now have A, and D is 10.66 , the average for the Han data set for Pwave velocities. We now solve for the remaining unknown coefficient $\mathrm{B}$.

Once we have coefficient B, we can use the equation that comes from integrating Figure 118 (shown in Figure 119) to find the slope of our equation between the original pressure and whatever new pressure we want. Our new velocity will simply be our original velocity plus or minus coefficient B times the slope we found in this step.

All these steps put together result in the following empirical equations:

P-wave velocities (eq. 6):

$$
\Delta V_{1-2}=\left[V_{1}-\frac{V_{1}-\left(.64 \ln P_{e 1}+2.53\right)}{-.12 \ln P_{e 1}+.54}\right]\left[\frac{-1.45\left(e^{-5.71 P_{e 2}}-e^{-5.71 P_{e 1}}\right)}{P_{e 1}-e^{-10.66 P_{e 1}}+1}\right]
$$

S-wave velocities (eq. 7):

$$
\Delta V_{1-2}=\left[V_{1}-\frac{V_{1}-\left(.35 \ln P_{e 1}+1.39\right)}{-.09 \ln P_{e 1}+.63}\right]\left[\frac{-1.44\left(e^{-5.39 P_{e 2}}-e^{-5.39 P_{e 1}}\right)}{P_{e 1}-e^{-10.54 P_{e 1}}+1}\right]
$$

These equations become more accurate as the step size between the two pressures decreases.

\section{Results}

This method was developed entirely from the Han dry data set by the procedures described above. All correlations between velocity and coefficients A, B, and D were made using all of Han's velocity values at effective pressures ranging from $.10 \mathrm{kbar}$ to $.50 \mathrm{kbar}$ (we originally included the $.05 \mathrm{kbar}$ point to build our method, but we found that our empirical equation was not accurate for pressures below .10 kbar. After redoing the method based only on the data between .10 and $.50 \mathrm{kbar}$, we found our equation could more accurately predict velocities in this range.) For this section, will we only discuss our results relating to P-wave velocity predictions, since the S-wave, bulk modulus and shear modulus results were done in a similar fashion to the $\mathrm{P}$-wave velocity. At the conclusion of this section, we will give results for these remaining three parameters, and discuss any difference in these results and the P-wave results.

The first test to see if this method works is to see how accurately it can predict the entire data set when only given the initial information of one velocity at a certain pressure for each rock in the data set. This means that if we were given the P-wave (or S-wave) velocity at $.20 \mathrm{kbar}$ for each sample in the data set, we should be able to accurately predict the P-wave velocity at all other pressures $(.05, .10, .30, .40, .50)$ for each sample in the data set. For each sample, we took the difference between our prediction and the actual velocity value and squared it, allowing us to measure the error in our predictions (Figure 120). 

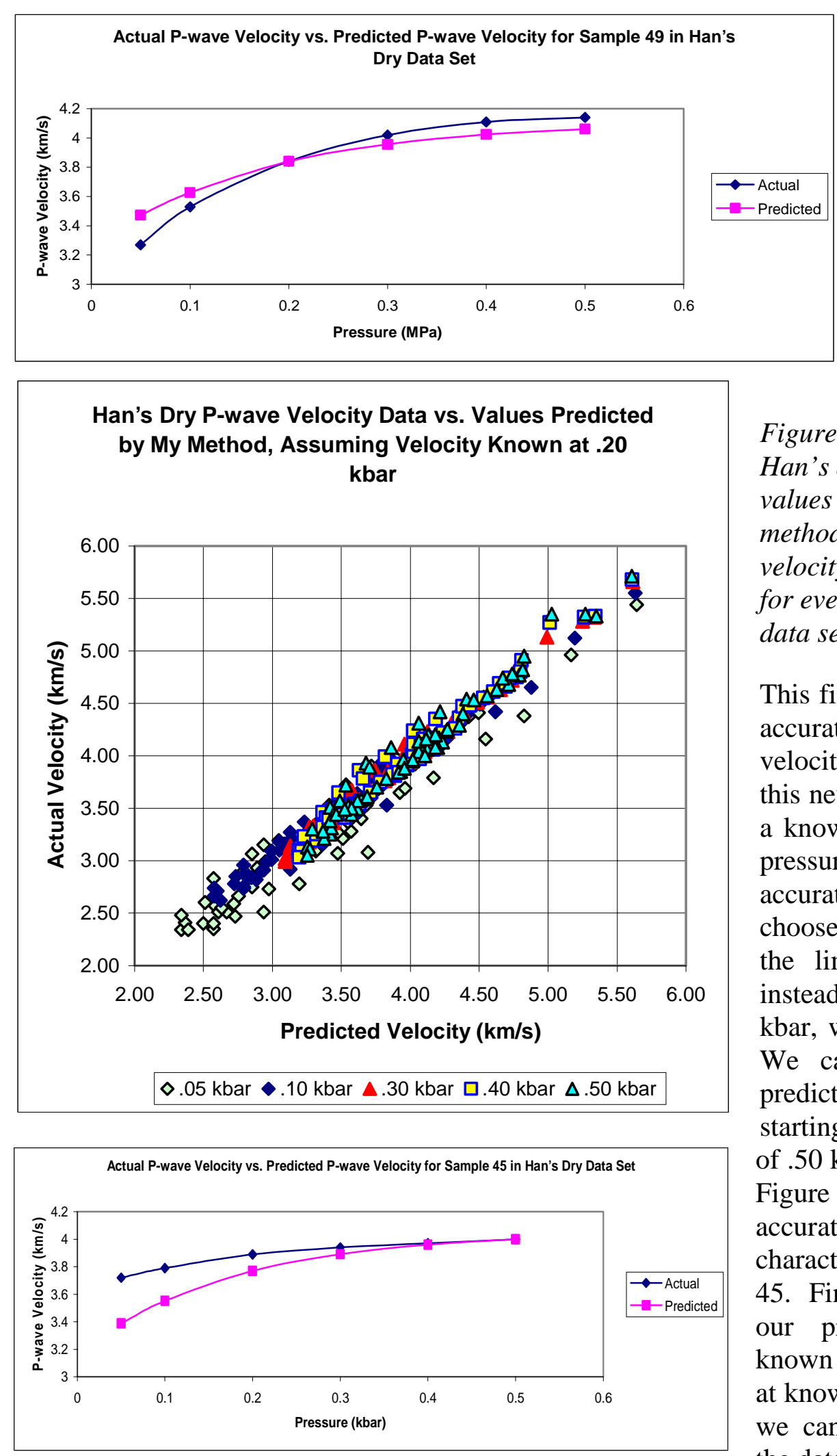

Figure 122: Plot of data for sample 45.
Figure 120: Plot of

data for sample 49.

The average error values at each pressure for the entire data set, along with the average overall error (just the sum of the average errors) is shown in Figure 121.

Figure 121: Actual values in Han's dry P-wave data set $v$ s. values predicted by my method, assuming a known velocity at $.20 \mathrm{kbar}$. This is for every sample in the entire data set.

This figure shows that we can accurately predict the P-wave velocities of this data set with this new method if we assume a known velocity at a certain pressure. However, how accurate is this method if we choose a pressure that is near the limits of our data set, instead of a pressure like .20 kbar, which is in the middle? We can generate a set of predicted velocity values starting with a known velocity of $.50 \mathrm{kbar}$ instead of $.20 \mathrm{kbar}$. Figure 122 shows how accurate this prediction is for a characteristic sample, sample 45. Finally, just as we did for our predictions starting at known velocities at $.20 \mathrm{kbar}$, at known velocities at $.50 \mathrm{kbar}$ we can analyze the error for the data set (Figure 123). 


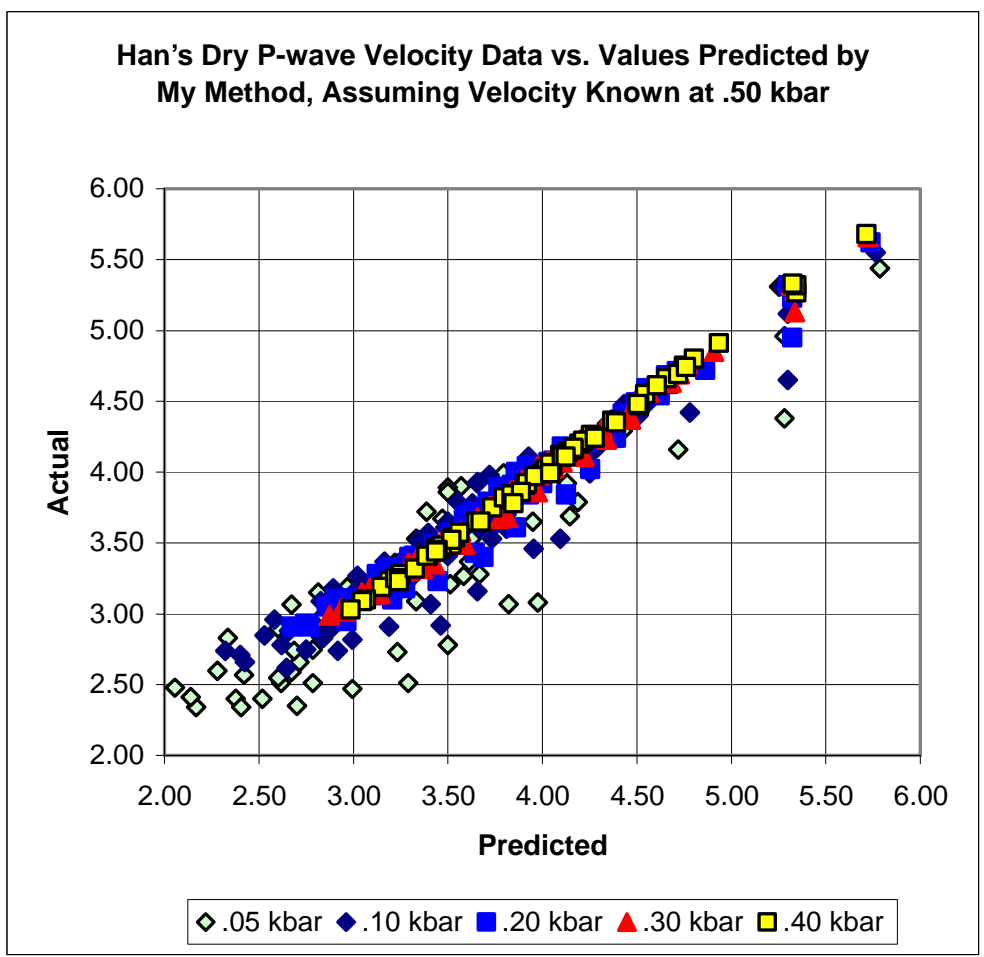

Figure 123: Actual values in Han's dry P-wave data set vs. values predicted by my method, assuming a known velocity at $.50 \mathrm{kbar}$. This is for every sample in the entire data set.

From Figures 120 and 123, it can be seen that, generally, the error increases as the predictions are made with increasing differences in pressure. Comparing the residual values at $.05 \mathrm{kbar}$ in these tables to the residual values at other pressures in the tables, and to the total residual values, it can be seen that the error at the $.05 \mathrm{kbar}$ point is significantly higher than the error at any other pressure. By removing the $.05 \mathrm{kbar}$ point, the error in the .20 kbar original pressure drops from .0749 to .0333 , a decrease in error of 56 percent. For the .50 kbar original pressure, this decrease in error is from .1862 to .0748 , or 60 percent. From these results, the conclusion is made that this method does not accurately predict velocities down to $.05 \mathrm{kbar}$. This conclusion is very similar to that of Bentley et al, 2000, who also concluded that his method is valid only at effective pressures greater than $.10 \mathrm{kbar}$.

This method seems to accurately predict velocities using the data set that it was developed for. This was a good internal check on the method, but to truly prove useful, we need to see how accurately this method can predict velocities given a new data set. The data set used to do this is from Freund, 1992. Freund's data set contained dry velocity measurements at pressures ranging from $.08 \mathrm{kbar}$ up to $3.0 \mathrm{kbar}$. Effective pressures in a reservoir generally range from near $0 \mathrm{kbar}$ up to about $.50 \mathrm{kbar}$, and our method was based on data to $.50 \mathrm{kbar}$. For these reasons, we will only test our method on the $.08, .24, .40$, and .60 kbar pressures in Freund's data.

To test our method on Freund's data, we first assumed that we only knew the velocities at the .24 kbar pressure. From this central pressure, we predicted velocities up to $.60 \mathrm{kbar}$ and down to .08 kbar, and compared them to the actual values. After this, we started with the velocities at .60 kbar as known, and predicted velocities down to $.08 \mathrm{kbar}$, to check how accurate our method would be when starting at the edge of the data we're interested in. A plot of the predicted values vs. the actual values for both cases are shown in Figure 124 and Figure 125.

Our conclusion from Han's data about predictions below a pressure of .10 kbar being inaccurate holds for Freund's data as well. The total error reduces from .2809 per sample to .1243 per sample (a decrease of 56 percent) for the $.24 \mathrm{kbar}$ starting point, and for the $.60 \mathrm{kbar}$ starting point, it decreases from .7255 per sample to .1430 per sample (a decrease of 80 percent). This reinforces our conclusion that this method is not accurate below $.10 \mathrm{kbar}$. 


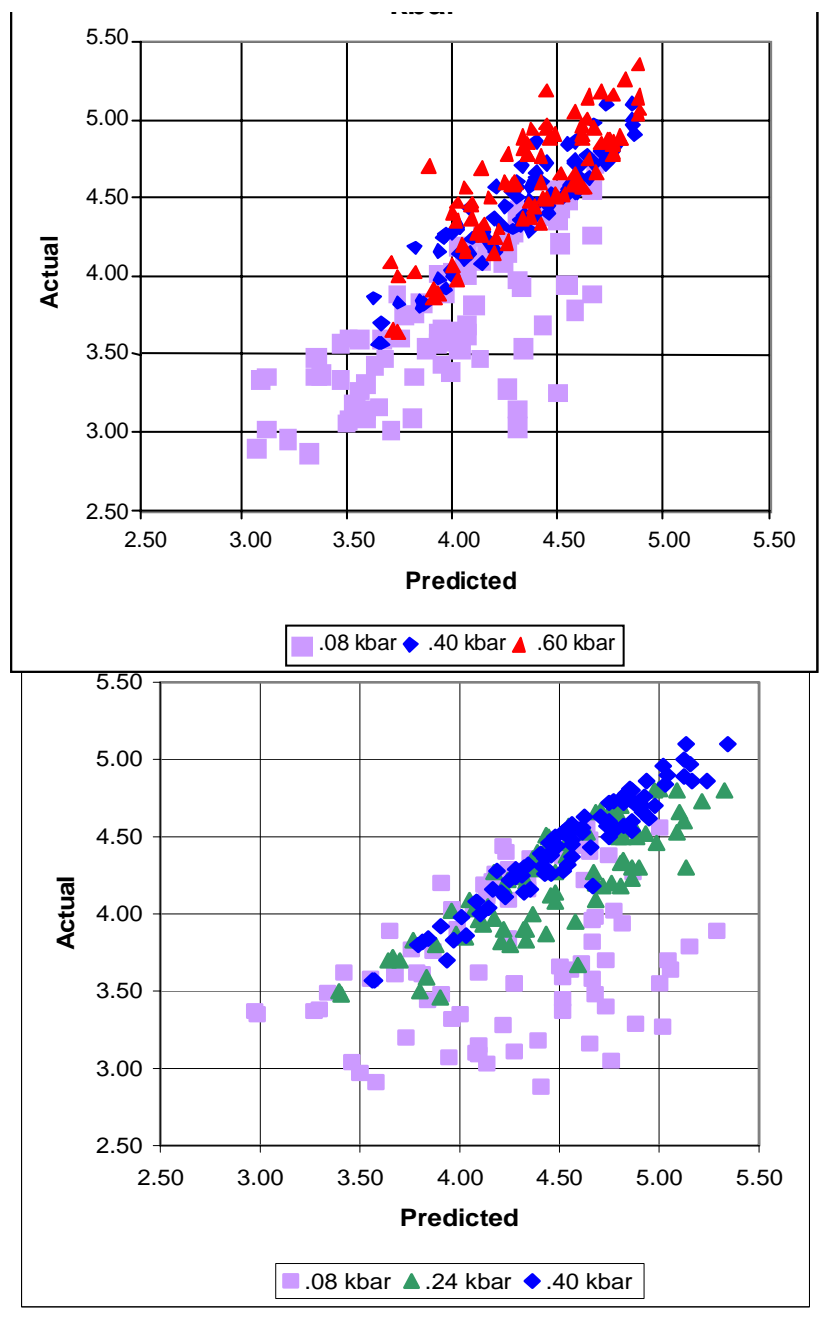

Figure 124: Actual values in Freund's dry Pwave data set vs. predicted values, assuming a known velocity at 24 kbar. This is for pressures .08, .40 and .60 for every rock sample in the entire data set.

Figure 125: Actual values in Freund's dry Pwave data set vs. predicted values, assuming a known velocity at $.60 \mathrm{kbar}$. This is for pressures .08, .24, and .40 for every sample in the entire data set.

But, looking at figures 124 and 125, we can see that predictions for Freund's data are much less accurate than the predictions for Han's data. This is to be expected for a few reasons. First, the method was developed empirically for Han's data, so it should fit this data set better than an outside data set like Freund's. Second, we're looking at a greater range in Freund's data - all the way up to $.60 \mathrm{kbar}$ - and as we said before, the accuracy of the predictions decrease the further from a known velocity you go. This being said, the best way we can determine how accurate our method is would be to compare it to other methods. Many have worked on this problem to develop an empirical equation that would predict velocity, so a comparison to these equations can show how useful this new method is.

\section{Landro's Method}

Another equation that predicts velocity changes due to pressure changes is described by Landro, 2001. Landro's technique utilized the equation

$$
\frac{\Delta \alpha}{\alpha} \approx k_{\alpha} \Delta S+l_{\alpha} \Delta P+m_{\alpha} \Delta P^{2} \text { (eq. 8) }
$$

where $\alpha$ is $\mathrm{P}$-wave velocity, $\Delta \mathrm{S}$ is change in oil saturation, $\Delta \mathrm{P}$ is change in net pressure, and $\mathrm{k}_{\alpha}$, $1_{\alpha}$, and $\mathrm{m}_{\alpha}$ are empirical parameters estimated from saturation and pressure curves from laboratory studies of core samples (Landro, 2001). Note that the change in net pressure for Landro's equation must be entered with the units of $\mathrm{MPa}(1 \mathrm{kbar}=100 \mathrm{MPa})$. A similar 
equation exists for S-wave velocities also, with slightly different empirical parameters. If we want to look at pressure effects alone (instead of pressure and fluid effects), we can simplify the equation to:

$$
\frac{\Delta \alpha}{\alpha} \approx l_{\alpha} \Delta P+m_{\alpha} \Delta P^{2} \text { (eq. 9) }
$$

Empirical parameters $1_{\alpha}$ and $\mathrm{m}_{\alpha}$ are solved for by plotting change in P-wave velocity vs. effective pressure, giving a curved line (Figure 126). A second order polynomial can be fit to this curved line - the coefficient in front of the $\mathrm{x}^{2}$ term is $\mathrm{m}_{\alpha}$, and the coefficient in front of the $\mathrm{x}$ term is $1_{\alpha}$. While $1_{\alpha}$ is positive, $\mathrm{m}_{\alpha}$ is usually negative.

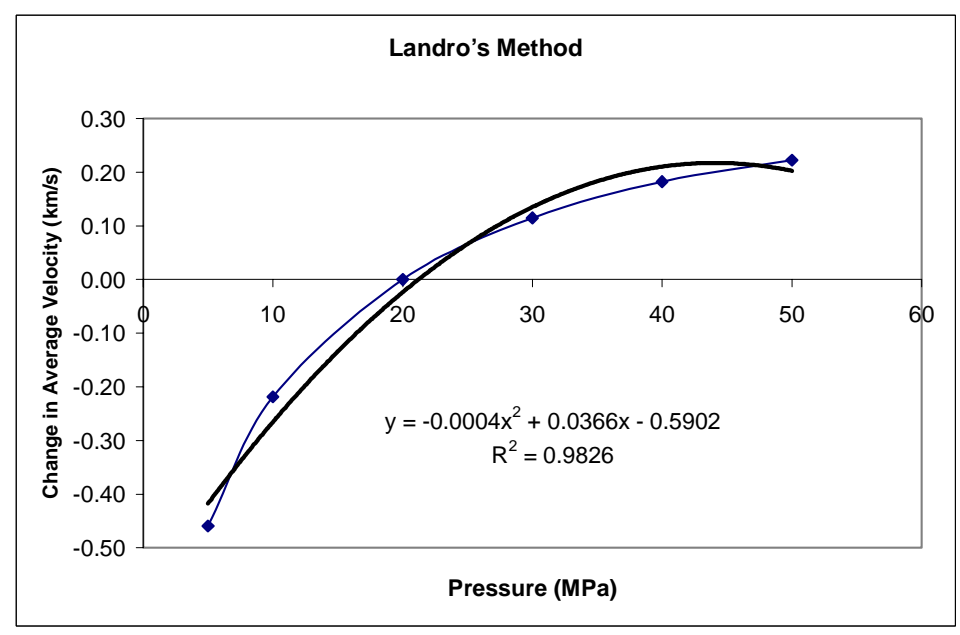

Figure 126: Changes between two pressures for the average velocities of the entire Han data set. Coefficient $l_{\alpha}$ is .0366 and $m_{\alpha}$ is .0004 .

Although Landro intended his method to be used for specific rock samples, we have applied it to an aggregate set of velocities from all the samples in the Han data set, assuming a known velocity at a pressure of $.20 \mathrm{kbar}$. The accuracy of this method can be compared with

the accuracy of our new method. Landro's method is accurate, but for this group of samples our method is a significant improvement. These results can be explained because our implementation of Landro's method uses a best fit polynomial from the entire data set to predict all velocity changes. This means that the magnitude of the velocity change is not dependent upon the original velocity. This point will be illustrated in the next section.

Also, it's important to note that Landro developed this equation based on the assumption that several core measurements already exist. In order to find coefficients $1_{\alpha}$ and $m_{\alpha}$ and solve for new rock velocities, velocity measurements at various pressures must be available for rocks in the area of interest. Our method only requires one known velocity at one known pressure to predict its velocities at other pressures.

\section{Bentley's Method}

Both Bentley's method (eq. 2 and eq. 3) and our method are empirically derived equations based on Han's data. However, Bentley et. al. used Han's wet rock data, and did not include any data above pressures of .40 kbar. Our method used Han's dry rock data, and also included pressures up to $.50 \mathrm{kbar}$ (Han, personal communication).

There is another significant difference between Bentley's method and our method. This difference is most evident at the highest velocities on Figure 126. Looking closely at the upper right velocities in this figure, it can be observed that the predicted velocity is actually increasing as the pressure decreases. This is because, according to the empirical method we developed, 
velocity changes decrease linearly as original velocity increases. At about $5.6 \mathrm{~km} / \mathrm{s}$ (depending on the original pressure), the velocity changes predicted by our method hit zero. At velocities higher than this, the predicted velocity changes will be negative, suggesting that velocity would actually decrease with a pressure increase. This is a limitation in our method, and allows our method to be applied to rocks only with velocities under about $5.6 \mathrm{~km} / \mathrm{s}$.

Figure 127 shows how our method and Bentley's method predict velocity changes for different original velocities (this figure assumes a starting pressure of .20 kbar), and compares them with the measured velocity changes in Han's data. The method developed in this paper shows a linear relationship, while Bentley's method shows a relationship roughly in the shape of a hyperbola. Interestingly, the relationship for Bentley's method does not produce a well defined function. This is caused because in Bentley's method, predicted velocity changes are not dependent on Pwave velocities alone, but also on S-wave velocities. Bentley's original equations were developed for bulk modulus and shear modulus, each of which is dependent on both P-wave and S-wave velocities.

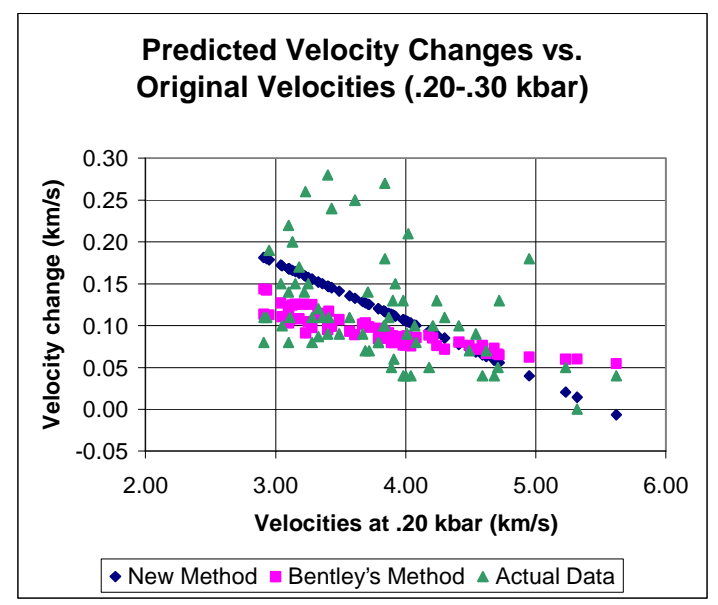

Figure 127: Comparing velocity change predictions between our new method and Bentley's method.

The original pressure of .20 kbar and pressure change between .20 and .30 kbar are arbitrarily chosen to use in the respective empirical equations. Other pressure values would alter the placement of the data slightly, but the same trends would still be observed. This figure uses P-wave velocities only.

This leads into another interesting aspect of Bentley's method. Bentley's method predicts changes in bulk modulus and shear modulus, regardless of what the original modulus is. Bentley's method is dependent only of the original pressure - the original modulus has no effect on further modulus predictions, meaning that the change in modulus predicted by Bentley's method is always the same for a given pressure and change in pressure. The method presented in this paper takes into account the original modulus - that is, changes in modulus predicted for a sample with a high known modulus would be different than changes predicted for a sample with a low known modulus at a certain pressure. Our new method predicts more realistic modulus changes compared to Bentley's. However, as we noted earlier, this appendix again shows that our method can predict negative changes in velocity or modulus at high velocity samples. So our method more accurately predicts modulus changes, but just as with high velocities, a high modulus can lead to unrealistic predictions.

Therefore, Bentley's method is preferred over our method at higher velocities, but our method can be used when only P-wave velocities are known, compared to Bentley's method that requires both P-waves and S-waves, and our method predicts modulus changes more realistically. The question that this leads to is: at what velocities is it better to use Bentley's method, and when is it better to use this new method? To examine this, we plotted our velocity predictions and 
Bentley's velocity predictions versus actual velocities for characteristic samples exhibiting high, medium and low original P-wave velocities (Figures 128, 129 and 130). From these figures, we make the conclusion that Bentley's method is more accurate at higher velocities (above about 5 $\mathrm{km} / \mathrm{s}$ ), but our method is more accurate for original velocities that are lower (below about 4 $\mathrm{km} / \mathrm{s}$ )

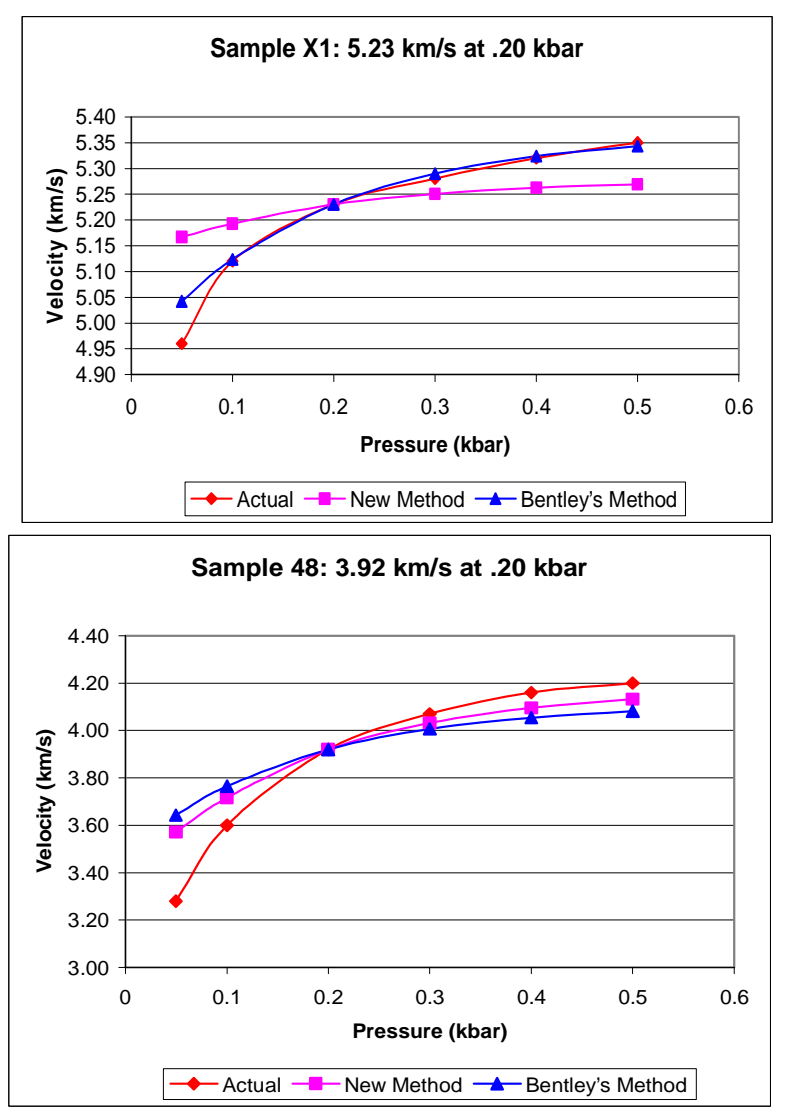

Figure 128: For this typical high velocity sample, Bentley's method predicts velocity changes more accurately than our new method. The new method predicts values that are too low for the slope of the curve at high velocities.

Figure 129: The typical medium velocity sample shows that the new method is more accurate than Bentley's method in this situation.

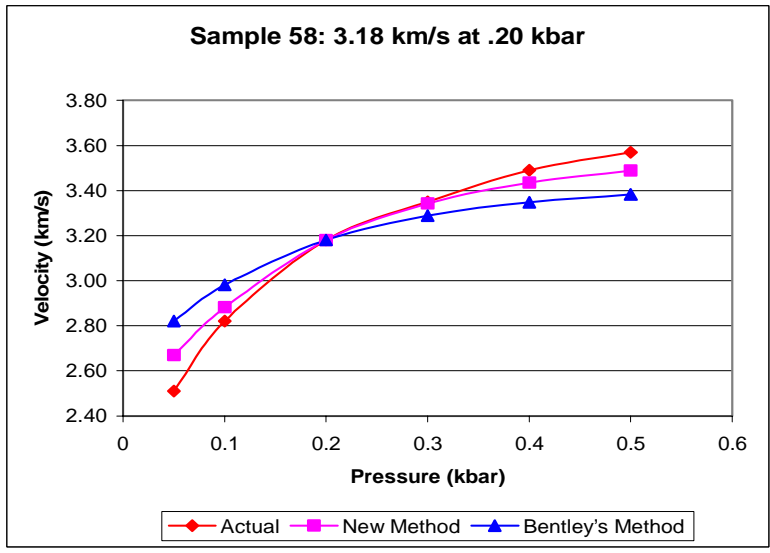

Figure 130: The new method predicts the actual velocity values even more accurately at this typical low velocity value.

\section{Comparison of Results}

We will compare tables showing total residual for this new method for P-wave, S-wave, bulk modulus, and shear modulus, for both the Freund data set and the Han data set. These will be compared to Bentley's method. Also, plots of predicted vs. actual values for all four parameters 
will be shown for both methods. The S-wave values for our method were calculated in the same way that we calculated the P-wave values. Our predictions for shear and bulk modulus were done two ways: 1 . Simply by converting our predicted P and S-waves to shear and bulk modulus and 2. Building an entire empirical equation (solving for coefficients A, B, and D) by using bulk modulus and shear modulus values alone. The results showed that it was significantly more accurate to predict bulk and shear modulus by the first method - directly converting our P and Swave predictions to shear and bulk modulus.

Two other pieces of data that we have for both the Han and Freund data set are porosity and clay content. These are also two parameters that many have often attempted to correlate to observed velocity changes. Both porosity and clay content in the Freund data show no correlation between error and porosity. However, Figure 131 shows clay content vs. residual error and displays a strong relationship. It's obvious from this figure that as clay content increases, the error in prediction tends to decrease. Particularly, sands with a clay content below 30 percent seem to have a much higher error. This observation is consistent for both this paper's predictions and Bentley's equation's predictions. Residual error vs. clay content was plotted for Han's data (Figure 132), but no relationship similar to that in the Freund data was observed.

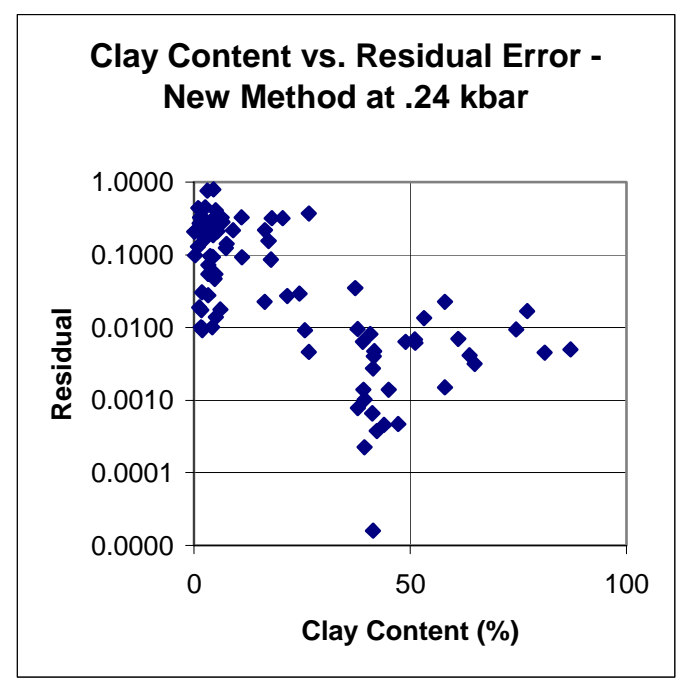

Figure 131: Clay Content vs. residual error for Freund's P-wave data is shown here. Samples with clay content below about 30 percent show a much higher error.

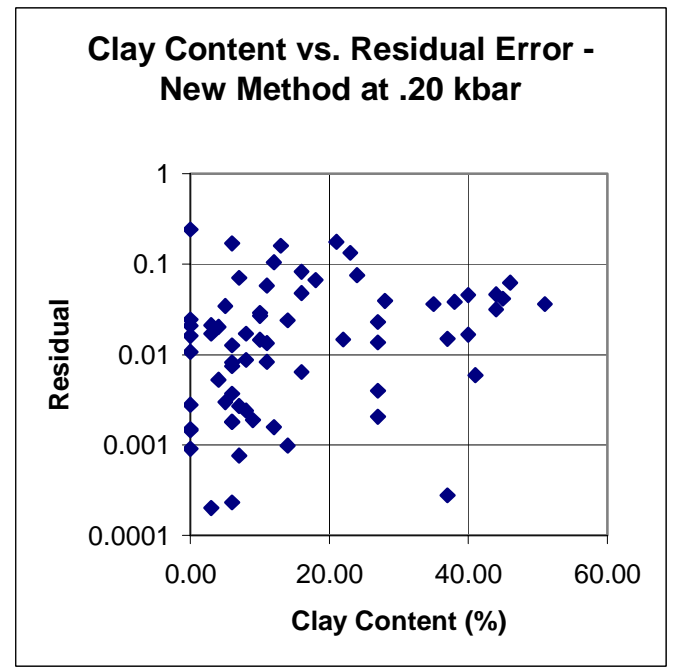

Figure 132: Clay Content vs. $R^{2}$ error (residual) for Han's P-wave data is shown here. Unlike the Freund data set, the magnitude of error does not seem to be affected by clay content.

\section{Summary of Results}

1. For both P-wave and S-wave velocities, the method presented in this paper is more accurate than Bentley's method when starting at lower pressures and predicting to higher pressures. However, when starting at higher pressure ranges (.50 or .60 kbar) and predicting to lower pressures, Bentley's method is more accurate. 
2. In almost every case, the new method presented here is more accurate than Bentley's method in predicting bulk modulus values. Bentley's method is more accurate in almost every case at predicting shear modulus, though. This seems to difficult to explain, since differences in Bentley's method and this method only depend on starting pressure for velocities. One possibility may be that Bentley's velocity predictions are compounded when converting to bulk modulus. Bulk modulus is calculated in part by subtracting S-wave velocity from P-wave velocity. If estimates for $\mathrm{P}$-wave velocity were too high, and estimates for S-wave velocity too low, then the bulk modulus would be significantly overestimated. The error in bulk modulus estimates in this case would be greater than if velocity predictions overestimated (or underestimated) the velocity in both cases.

3. Clay content and error in velocity predictions are strongly related in the Freund data set. The trend shows that both velocity predictions become less reliable in cleaner sands. Han's data set shows that this isn't always the case - the clean sands in this data set show no more error than other samples.

4. Comparing AVO effects observed in an actual producing field (the Teal South study, previously reported) to those predicted with the inclusion of pressure effects successfully demonstrated that velocity changes due to pressure variation must be considered. Pressure changes can have as significant an effect on velocity as fluid changes do, and are important to consider in any reservoir characterization. 


\section{The Dynamic Fluid Method}

The Dynamic Fluid Method (also previously translated as the 'Dislocation Fluid Model') was developed by Dr. Vladimir Pisetski of the Urals Mining Academy, in Ekaterinburg, Russia. The technique is patented, and Pisetski's USA-based company, TransSeismic International (TSI), is licensed to perform the DFM analysis. The DFM approach is both a computational technique and a conceptual interpretational technique. In this report, we are primarily concerned with the computations. Many additional details can be found in their complete report in the appendix.

The DFM computations are based on seismic attributes, particularly the instantaneous frequency and instantaneous amplitude, combined in such a way as to remove the average values along horizons or time-slices, and to enhance those aspects presumed to result from open cracks. In essence, the value of instantaneous amplitude (or frequency) is normalized at every point along the horizon (or time slice) by the average value along that horizon. These relative values are presumed to correspond more to the differences in physical state of the rock along the horizon than the original total value, which in turn correspond more to the change in lithology vertically in the section. That is, the lithologic differences generally sought through reflection seismology are in effect removed, by normalizing by the average values (along horizons or time-slices) of the attributes. The DFM method presumes that those differences remaining are indicative of open fractures, which in turn result from 'decompression' of blocks of rock, due mostly to unloading and differential compaction.

In summary, the 3-D seismic volume was first processed using DFM method to determine the total ground pressure $P_{i}$ :

$P_{i}=P_{i g}+P_{i d}$

where $P_{i g}$ is the pressure caused by gravity load, $P_{i d}$ is the anomalous pressure caused by the modern movements and by anomalous fluid pressure. The second step was interpretation of the parameters of total ground pressure and finding anomalous pressure zones. The sequence of seismic data processing is summarized as follows:

- Interpretation of seismic horizons;

- Determination of the values of instantaneous amplitude and frequency at every sample point;

- Determination of the average estimates of instantaneous amplitude and frequency for given horizon;

- Calculation of the value $\theta_{i}$ at each reflecting point at the interface (an equation for $\theta_{i}$ is given in Appendix A) ;

- Normalization of the calculated values $\theta_{i}$ in the range between the minimum and maximum values.

The specific attributes of instantaneous frequency and amplitude are combined in the following equation 


$$
\theta_{i}=\left[\frac{A_{i}}{\tilde{A}_{i}}\right]^{b}\left[\frac{\tilde{\omega}_{i}}{\omega_{i}}\right]^{c}
$$

where the subscript $i$ refers to the $i$-th reflection point, A refers to the instantaneous amplitude, $\omega$ refers to the instantaneous frequency, and the tilde $\sim$ indicates an average value. The powers $b$ and $\mathrm{c}$ are fitted to the data by the investigator, in an effort to best make the term $\theta$ correspond to effective pressure as indicated by other information. Their report, submitted separately, provides more details on the DFM procedure to relate this parameter, quantitatively, to pressure.

TSI was invited to perform their DFM analysis on three of the fields described in this report (Stratton, Boonsville, and Wamsutter), and an additional data set not specifically studied by Michigan Tech, but studied by the Texas Bureau of Economic Geology (Waha). At the start of this project, microfracturing and pressure differences were believed to be a significant factor in the production at Wamsutter and Waha, as well as portions of Boonsville. Stratton was believed to be entirely a stratigraphic play. TSI has interpreted their DFM results in terms of the DFM theoretical framework which places great importance on differential vertical compaction during diagenesis and later vertical tectonics and associated compression and decompression.

We found the similarity of the DFM results for the Lewis shale interval in Wamsutter to our differential acoustic impedance analysis to be intriguing, and suspect that both techniqes are revealing features directly related to pressure variations. Results from the other two data sets studied by both groups were not obviously comparable, although TSI found strong correlations between their results and the production data that was available to them during their study. Three of the data sets studied by TSI are available in the public domain, and other investigators are invited to compare their observations with those of TSI as described in their reports, included in the appendix. 


\section{Conclusions}

In order to make judicial use of seismic attributes for reservoir characterization, one must take into account the seismic petrophysical aspects of the goals of the characterization in designing the attribute suite, or even the processing flow leading to the attribute suire. The use of acoustic impedance from inverted seismic data is often warranted, and leads to additional insight for layer, and not interface, properties, but care must be taken to avoid confusing elastic (offsetdependent) impedance with acoustic impedance.

With the use of well-designed attributes, one can often determine properties of reservoir intervals that are of interest. Care must be taken whenever horizon-based attributes are used without tracking the peak or trough on which they are based. For subtle features, or those not readily amenable to seismic detection, specially designed attributes can be of tremendous value.

The attributes which we have found most valuable include acoustic impedance from trace inversion and amplitude. In order to use these attributes, however, a realistic expectation of the various responses for different reservoir conditions must be available. Then, the use of these attributes may become apparent. The straightforward application of attributes without a firm grasp of the physics involved is not usually recommended - even in the 'simplest' environment of a bright-spot in the Gulf of Mexico, we found that interpretation of the magnitude of the bright spot (over time) could not be conducted without a thorough understanding of the rock physics and its effect on the stacked section, after amplitude-versus-offset effects were included.

Calibration of seismic attributes must be undertaken in every field study, independently, in order to have confidence in the interpretations that result. 


\section{Acknowledgments}

The Energy Research Clearing House, particularly Roger Entralgo and Gene Sparkman, and the members of its Teal South 4D-4C consortium, provided the Teal South data and helpful discussions. Preliminary models were aided by advice provided by J. Ashbaugh and P. Flemings of Penn State. Legacy streamer data was provided by Diamond Geophysical, and Phase I and Phase II data was provided by CGG; W. Haggard and S. Spitz provided additional details and specially formatted data for our use. Assistance in performing the inversion for acoustic impedance was provided by B. Rees and D. Graham of Jason Geosystems.

$\mathrm{BP}$ and its predecessors have generously provided the Wamsutter data set for our use, and have been extremely helpful in discussions and advice. We gratefully acknowledge their support of this project.

Software used in this project has been graciously provided by Landmark Graphics, Jason Geosystems, Schlumberger-GeoQuest, Hampson-Russell, Paradigm, Scott-Pickford, Mercury International, Paradigm Geophysical, Seismic Micro-Technology, and GXTechnology.

All interpretations presented in this report are those of the authors, and not necessarily those of the people or companies that support this research. The work of TransSeismic International is their own work, and the interpretations and conclusions made by them are not necessarily those of Michigan Tech nor of the other people, companies, or government agencies that supported the work. Responsibility for all errors rests with the authors. [Additional disclaimer is presented at the start of this report.] 


\section{References}

Batzle, M. and Wang, Z., 1992, Seismic properties of pore fluids, Geophysics, 57, 1396-1408.

Backus, G, 1962, Long-wave elastic anisotropy in transversely isotropic media: Geophysics, v. 44, p. 896-917.

Bahorich M. and, S. Farmer, 1995, 3-D seismic discontinuity for faults and stratigraphic features: the coherence cube: The Leading Edge, v. 14, p. 1053-1058.

Bentley, L.R., Zhang, J., and Lu, H.-X., 2000, Evaluating feasibility of seismic fluid monitoring: 70th Ann. Internat. Mtg., Soc. Expl. Geophys., Expanded Abstracts

Brown, A. R., 1996, Interpretation of 3-D seismic data (4th edition): AAPG Memoir 42, published by AAPG, Tulsa, OK.

Brown, A., 2001, Understanding seismic attributes: Geophysics, 66, 47-48.

Carr, D. L., R. A. Johns, and R. Y. Elphick, 1997, High-resolution reservoir characterization of midcontinent sandstones using wireline resistivity imaging in Boonsville (Bend Conglomerate) gas field, Fort Worth basin, Texas: The Log Analyst, v. 38, n. 5, p. 54- 69.

Chen C., 1996, Fuzzy logic and neural network handbook, McGraw-Hill, Inc.

Chen, Q. and S. Sidney, 1997, Seismic attribute technology for reservoir forecasting and monitoring, The Leading Edge, May, p. 445-456.

Chi, C., J. Mendel, and D. Hampson, 1984, A computationally fast approach to maximumlikelihood deconvolution: Geophysics, v. 49, v. 5, p. 550-565.

Chopra, S., 2001, Integrating coherence cube imaging and seismic inversion, The Leading Edge, April, p. 354-362.

Coleman, J. M. and D. B. Prior, 1982, Delta environments of deposition, in Sandstone depositional environments edited by P. A. Scholle and D. Spearing, published by AAPG, Tulsa, OK, p. 139-170.

Cooke, D. and W. A. Schneider, 1983, Generalized linear inversion of reflection seismic data: Geophysics, v. 48, n. 6, p. 665-676.

Cooke, D., A. Sena, and G. O'Donnell, T. Muryanto, and V. Ball, 1999, What is the best Seismic attribute for quantitative seismic reservoir characterization?: SEG 1999 Expanded Abstract.

Craft, B.C., Hawkins, M., 1991, Applied Petroleum Reservoir Engineering, Prentice Hall

Ebrom, D., P. Krail, D. Ridyard, and L. Scott, 1998, 4-C/4-D at Teal South, The Leading Edge.

Dillen, M.W.P, Cruts, H.M.A., Groenenboom, J., Fokkema, J.T., and Duijndam, A.J.W., 1999, Ultrasonic velocity and shear-wave splitting behavior of a Colton sandstone under a changing triaxial stress: Geophysics, 64, 5, 1603-1607.

Dvorkin, J., Nur, A., and Chaika, C., 1996, Stress sensitivity of sandstones: Geophysics, 61, 2, 444-455.

Dvorkin, J. and Prasad, M., 1999, Elasticity of marine sediments: Rock physics modeling: Geophysical Research Letters, 26, 12, 1781-1784. 
Dvorkin, J., Nolen-Hoeksema, R., and Nur. A, 1994, The squirt-flow mechanism: Macroscopic description: Geophysics, 59, 3, 428-438.

Eberhart-Phillips, D., Han, D-H., and Zoback, M.D., 1989, Empirical relationships among seismic velocity, effective pressure, porosity, and clay content in sandstone: Geophysics, 54, 1, 82-89.

Ebrom, D., P. Krail, D. Ridyard, and L. Scott, 1998, 4-C/4-D at Teal South, The Leading Edge.

Emery, D. and K. Myers, 1996, Sequence stratigraphy, published by Blackwell Science Ltd., p. 61- 177.

Freund, D., 1992, Ultrasonic compressional and shear velocities in dry clastic rocks as a function of porosity, clay content, and confining pressure: Geophys. J. Int., 108, 125-135.

Gastaldi, C., D. Roy, P. Doyen, and L. Den Boer, 1998, Using Bayesian simulations to predict reservoir thickness under tuning conditions: The Leading Edge, n. 4, p. 589- 593.

Glover, G., 1982, A study of the Bend Conglomerate in S. E. Maryetta area, Boonsville field, Jack county, Texas: Petroleum Geology of the Fort Worth basin and Bend Arch area, edited by C. A. Martin, Dallas Geological Society, p. 353-354.

Gurney, K. and Wright M., 1992, A self-organizing neural network model of image velocity encoding, in Biological Cybernetics, v. 68, p.173-181.

Han, D-H., Nur, A., and Morgan, D., 1986, Effects of porosity and clay content on wave velocities in sandstones: Geophysics, 51, 11, 2093-2107.

Hardage, B. A., R. A. Levey, and V. Pendleton, etc., 1994, A 3-D seismic case history evaluating fluvially deposited thin-bed reservoirs in a gas-producing property: Geophysics, v. 59, n. 11, p. 1650-1665.

Hardage, B. A., R. A. Levey, V. Pendleton, 1994, A 3-D seismic case history evaluating fluvially deposited thin-bed reservoirs in a gas-producing property, Geophysics, v. 59, n.11, pp 16501665.

Hardage, B. A., J. L. Simmons, and Jr. D. E. Lancaster, etc., 1996a, Boonsville 3-D seismic data set: a technology transfer product generated as part of the secondary gas recovery project (GRI96/0182).

Hardage, B. A., D. L. Carr, D. E. Lancaster, et al., 1996b, 3-D seismic imaging and seismic attribute analysis of generic sequence deposited in low-accommodation conditions, 1996: Geophysics, v. 61, n. 5, p.1351-1362.

Hardage, B. A., D. L. Carr, D. E. Lancaster, et al.., 1996c, Secondary natural gas recovery: targeted applications for infield reservoir growth in midcontinent reservoirs, Boonsville field, Fort Worth basin, Texas: Gas Research Institute, Topical Report(GRI-95/0454.1-2), v. I and v. II.

Hoover, A. R., T. Burkhart, and P. B. Flemings, 1999, Reservoir and production analysis of the K40 sand, south Timbalier 295, offshore Louisiana, with comparison to time- lapse (4-D) seismic results: AAPG, Bulletin, v. 83, n. 10, p. 1624-1641. 
Horkowitz, K. O., and D. R. Davis, 1996, Seismic delineation of thin sandstone reservoirs in a shale-rich sequence using instantaneous frequency and reflection amplitude attributes from 3-D data, Texas Gulf Coast, in AAPG Studies in Geology no. 42 and SEG Geophysical Development Series No. 5, edited by P. Weimer and T. L. Davis, AAPG/SEG, Tulsa, OK., p. 35-44.

Hovem, J.M., 1995, Acoustic waves in finely layered media, Geophysics, 60, 1217-1221.

Jason Geoscience Workbench, Trace-Based Inversion and Modeling Training Manual, 1997.

Jaworowski, C., and Simon, R., 1995, Relating fractures in the eastern Greater Green River Basin to oil and gas fields: integrating field measurements, linear features, and digital geologic data: Wyoming Geological Association 1995 Field Conference Guide Book, 61-75.

Khaksar, A., and Griffiths, C.M., 1999, Influence of Effective Stress on the Acoustic Velocity and Log-Derived Porosity: SPE Reservoir Eval. \& Eng., 2, 1, 69-74.

Khaksar, A., Griffiths, C.M., and McCann, C., 1999, Compressional- and shear-wave velocities as a function of confining stress in dry sandstones: Geophysical Prospecting, 47, 487-508.

King, M.S., 1966, Wave velocities in rocks as a function of changes in overburden pressure and pore fluid staturants: Geophysics, 31, 1, 50-73.

Kohonen, T. 1990, Self-organizing map, Proceedings of the EEE, v.78, n.9, p.1464-1480.

Lahti, V. R. and W. F. Huber, 1982, The Atoka group (Pennsylvanian) of the Boonsville field area, north-central Texas: Petroleum Geology of the Fort Worth basin and Bend Arch area, edited by C. A. Martin, Dallas Geological Society, p. 377-399.

Landro, M., 2001, Discrimination between pressure and fluid saturation changes from time-lapse seismic data: Geophysics, 66, 3, 836-844.

Landro, M, 2001, Discrimination between pressure and fluid saturation changes from time lapse seismic data: 69th Ann. Internat. Mtg., Soc. Expl. Geophys., Expanded Abstracts

Lindsay, R., and Van Koughnet, R., 2001, Sequential Backus Averaging: Upscaling well logs to seismic wavelengths, TLE, Vol. 20, No.2, 188-191.

Liu Z. and J. Liu, 1998, Seismic-controlled nonlinear extrapolation of well parameters using neural networks: Geophysics, v. 63, n. 6, p. 2035-2041.

Lumley, D.E., Behrens, R.A. and Wang, Z., 1997, Assessing the technical risk of a 4-D seismic project, The Leading Edge, 16, 1287-1291.

Marion, D., Mukerji, T., and Mavko, G., 1995, Scale effects on velocity dispersion: From ray to effective medium theories in stratified media, Geophysics, 60, 1613-1619.

Marfurt K., R. Kirlin, S. Farmer and M. Bahorich, 1998, 3-D seismic attributes using a semblance-based coherency algorithm: Geophysics, v. 63, n. 4, p. 1150-1165.

Marfurt K., V. Sudhaker, A. Gersztenkorn, K. Crawford, and S. Nissen, 1999, Coherency calculations in the presence of structural dip: Geophysics, v. 64, n. 1, p. 104-111.

Martinsen, R.S., et al., 1995, Stratigraphy and lithofacies of the Almond Formation, Washakie and Great Divide Basins, Wyoming: Wyoming Geological Association 1995 Field Conference Guide Book, 297-310 
Mavko, G., Mukerji, T., and Dvorkin, J., 1998, The Rock Physics Handbook: Tools For Seismic Analysis In Porous Media, Cambridge University Press.

Mavko, G., Mukerji, T., Godfrey, N., 1995, Predicting stress-induced velocity anisotropy in rocks: Geophysics, 60, 4, 1081-1087.

Meadows, M., 2001, Enhancements to Landro's method for separating time-lapse pressure and saturation changes: 71st Ann. Internat. Mtg., Soc. Expl. Geophys., Expanded Abstracts

Merkel, R.H., Barree, R.D., and Towle, G., 2001, Seismic response of Gulf of Mexico reservoir rocks with variations in pressure and water saturation: The Leading Edge, 20, 3, 290-299.

Michelena, R. J., E. Gonzalez S., and M. Capello De P. 1998, Similarity analysis: A new tool to summarize seismic attributes information: The Leading Edge, v.17, n. 4, p. 545-548.

Murphy, W.F., Winkler, K.W., and Kleinberg, R.L., 1986, Acoustic relaxation in sedimentary rocks: Dependence on grain contacts and fluid saturation: Geophysics, 51, 3, 757-766.

Oldenburg, D. W., T. Scheuer and S. Levy, Recovery of the acoustic impedance from reflection seismograms: Geophysics, v. 48, n. 10, p. 1318-1337.

Parker, R., 2001, Identifying "Sweet Spots" in basin centered gas accumulations: Gas TIPS, June 2001

Partyka, G., J. Gridley, and J. Lopez, 1999, Interpretational applications of spectral decomposition in reservoir characterization: The Leading Edge, vol. 18, p. 353-360.

Pennington, W., 1993, Summary of petrophysical measurements for scaling of seismic properties: Colorado School of Mines Reservoir Characterization Project - Phase IV V, Sponsor Meeting Report, April 22, 1993.

Pennington, W. D., 2001, Reservoir geophysics, Geophysics, vol. 60, no. 1, p. 25-30.

Pennington, W., Acevedo, H., Haataja, J., and Minaeva, A., Seismic time-lapse surprise at Teal South: That little neighbor reservoir is leaking!: The Leading Edge, 20, 10, 1172-1175.

Pisetski, V., 1999, The Dynamic Fluid Method, The Leading Edge.

Poupon, M., K. Azbel, and J. Ingram, 1999, Integrating seismic facies and petro-acoustic moldering: World Oil magazine, June 1999, p. 75-80.

Prasad, M. and Dvorkin, J., 2001, Velocity to Porosity Transform in Marine Sediments, Petrophysics, 42, 5, 429-437.

Prasad, M. and Manghnani, M.H., 1997, Effects of pore and differential pressure on compressional wave velocity and quality factor in Berea and Michigan sandstones: Geophysics, $62,4,1163-1176$

Riel, P., 2000, The past, present, and future of quantitative reservoir characterization: The Leading Edge, no. 8, p. 878-881.

Rio, P., et. al., 1996, Velocity dispersion and upscaling in a laboratory-simulated VSP, Geophysics, 61, No.2, 584-593.

Roehler, H.W., 1990, Stratigraphy of the Mesaverde Group in the Central and Eastern Greater Green River Basin, Wyoming, Colorado, and Utah: U.S. Geological Survey Prof. Paper 1508. 
Russell B., 1988, Introduction to seismic inversion methods, S. N. Domenico, Series Editor Course Notes Series, Volume 2 (an SEG continuing education short course).

Salleh M. S. and S. Ronghe, Reservoir characterization on thin sands in south west Ampa 21 area (BLK11) using seismic inversion: SEG 1999 Expanded Abstracts.

Sturm, S.D., et al., 2000, Multi-disciplinary analysis of tight gas sandstone reservoirs, Almond Formation, Siberia Ridge Field, Greater Green River Basin: GRI report number GRI-00/0026.

Taner, M.T., and Sheriff, R.E., 1977, Application of amplitude, frequency and other attributes to stratigraphic and hydrocarbon determination, in C.E. Payton, ed., Seismic Stratigraphy: Applications to Hydrocarbon Exploration: AAPG Memoir 26, 301-327.

Taner, M.T., Koehler, F., and Sheriff, R.E., 1979, Complex seismic trace analysis, Geophysics, 44, 1041-1063.

Taylor, H, S. Banks, and J. McCoy, 1979, Deconvolution with the L1 norm: Geophysics, v. 44, p. 39-52.

Thompson, D. M., 1982, Atoka Group (Lower to Middle Pennsylvanian), Northern Fort Worth basin, Texas: Terrigenous depositional system, diagenesis, and reservoir distribution and quality (Investigation report No. 125).

Torres-Verdin C., M. Victoria, G., Merlrtin, and J. Pendrel, 1999, Trace-based and geostatistical inversion of 3-D seismic for thin-sand delineation: an application to San Jorge Basin, Argentina: SEG 1999 Expanded Abstracts.

Widess, M. B., 1973, How thin is a thin bed? Geophysics, v. 38, p. 1176-1180.

Yilmaz, O., 1987, Seismic data processing; published by SEG, (Investigations in Geophysics, Volume 2), Tulsa, OK.

White, J.E., 1983, Underground sound; application of seismic waves: Methods in Geochemistry and Geophysics, 18, 253.

Wyllie, M.R.J., Gardner, G.H.F., and Gregory, A.R., 1962, Studies of elastic wave attenuation in porous media: Geophysics, 27, 5, 569-589. 


\section{List of Acronyms and Abbreviations}

2D------------- Two dimensional

3D ------------- Three dimensional

BEG-------------- University of Texas Bureau of Economic Geology

CMP -----------Common Mid-Point

DOE ---------- United States Department of Energy

DFM -----------Dynamic Fluid Method

ERCH------------Energy Research Clearing House

MTU ------------- Michigan Technological University or Michigan Tech

NMO-------------Normal Move-out

SEG ------------ Society of Exploration Geophysicists

TSI ------------- TransSeismic International

USA-------------- United States of America 


\section{Appendices: Reports from TransSeismic International}

[The reports are reproduced as submitted by TransSeismic Internationa, with minor editing and reformatting only. They do not necessarily reflect the opinions of Michigan Technological University nor the US Dept of Energy. Editorial comments by MTU are in brackets and italics].

\section{DISCLAIMER}

This report was prepared as an account of work sponsored by an agency of the United States Government. Neither the United States nor any agency thereof, nor any of their employees, makes any warranty, express or implied, or assumes any legal liability or responsibility of the accuracy, completeness that its use would not infringe privately owned rights. Reference herein to any specific commercial product, process, or service by trade name, trademark, manufacturer, or otherwise does not necessarily constitute or imply its endorsement, recommendation, or favoring by the United States Government or any agency thereof. The views and opinions of authors expressed herein do not necessarily state or reflect those of the United States Government or any agency thereof.

\section{Stratton and Boonsville Fields}

The subcontractor ( Trans Seismic International, Inc., address: 1329 Clay Street, suite 4, San Francisco, CA 94109) retains all rights to the invention named the Dynamic Fluid Method and the software programs DYNAMIX (as well as its versions DYNAMIXt and DYNAMIXs), which were invented, developed and applied prior the commencement of work under the agreement \# 970821Z in the Stratton and Boonsville fields (Texas, the USA) as well as outside of the agreement \# 970821Z for other applications in different fields (e.g. White Tiger, Vietnam, Kaliningrad, CIS, Tartastan, CIS).

[United States Patent number 5,796,678 filed August $11^{\text {th }}, 1997$, as well as other continuationin-part patents are pending (with the filing date of August $11^{\text {th }}, 1997$ ). Patent title: "METHOD FOR DETERMINING THE PRESENCE OF FLUIDS IN A SUBTERRANEAN FORMATION", inventor: Dr. Vladimir B. Pisetski, Ekaterinburg, Russian Federation, assignee: Trans Seismic International, Inc., San Francisco, California, the USA. Method and techniques, outlined in the 10 claims of the patent and patents pending mentioned above cover the method and the techniques used in the data analysis under the agreement \# 970821Z. Also, please, note, that the Dynamic Fluid Method, as well as the algorithms and the software programs constituting DYNAMIX (as well as its versions DYNAMIXt and DYNAMIXs) have been applied ("inhouse" prior to the obtaining of the patent) in over 20 different fields since 1982.]

\section{INTRODUCTION}

The purpose of the work is to test the Dynamic Fluid Method-based technologies of processing and interpretation of 3D seismic data for identifying oil and gas deposits in the conditions of fluvial basins and in the regions of karst formations on the datasets from the South and North Texas (Stratton and Boonsville fields).

Involved in the projects: O.Voronin, V.Samsonov, Yu.Patrushev (processing), V.Pisetski (interpretation and report compilation), V.Kormiltsev, A.Ratushnyak (calculations and modeling), A.Minaeva (horizon picking, processing), E.Herzog (design and management). 


\section{ABSTRACT (EXECUTIVE SUMMARY)}

The process of hydrocarbon pool generation occurs in a generally accepted order: generation $=>$ migration $=>$ accumulation. Mostly, prediction of oil and gas resources is geared towards the last part in this chain, i.e. accumulation, - to finding traps of different types. The multitude of technologies for and approaches to the interpretation of seismic data compete in accuracy and reliability of the prediction of the traps of structural, stratigraphic, or lithological type. «Direct» indication of small and non-structural traps in complex sedimentation settings (including the conditions of fluvial and karst formations in the fields under consideration, i.e. Stratton and Boonsville) is not always possible with a traditional technology. The term "direct" refers to a technique of the prediction of petrophysical anomalies and those of a characteristic shape. Statistical methods are widely applied for the identification of such targets, as well as the techniques for the direct prediction of the acoustic impedance values with the use of calibration of well-logging data.

The DFM approach to analyzing seismic data is based on the understanding that the migration and, consequently, the accumulation of fluids are controlled by two main parameters, i.e. the permeability of the medium and the super-hydrostatic pressure (the Darcy's flow). If we direct our efforts to the prediction of these two parameters, we can then detect a closed contour of abnormal pressures (determining also the outline of a trap of any possible type), or areas that are most likely areas of fluid accumulation based on the derived parameters of fluid flow (the spatial distribution of pressure or permeability determined on the basis of pressure values). In essence, the total pressure and its variation in the geological time scale determine the fluid dynamics and make the fluid migrate from the regions of a compression into the regions of low pressures (the areas of decompression). Why do we benefit from locating regions of low pressures?

1. Any heterogeneity (geometrical or lithological) of the medium distorts the normal total ground pressure within the outline of this heterogeneity, as well as rather far beyond it, in overlying and underlying formations. Therefore, the region of an anomalous pressure, as a rule, exceeds several times the size of the heterogeneity source, and this region can also be detected on the properties of the seismic horizons distant from the target. For instance, if a sandy body (a channel) is located in a plastic formation (clayey deposits), then, a region of anomalous compression will be located above the channel, and a region of decompression will be located below it. Both anomalous regions will be several times wider than the transverse size of the channel. Figuratively speaking, the channel will throw a peculiar shadow to the overlying and underlying horizons, and this shadow will repeat the shape of the channel with some enlargement.

2. Heterogeneities of some formation type (traps) originate from certain dynamics of the sedimentary basin caused by the active impact of the basin at the moment of generation of this heterogeneity. The scheme of this dynamics or its peculiarities should be preserved in the modern conditions of a basin and can help us in identifying regions of the medium where generation of the traps of this or that type is most probable. The active region of the medium (the region of decompression or compression) is determined in a given moment of time (the geological time) by the anomalous pressure. If we find a current estimate of the anomalous pressure, we can then define the contour of a possible 
prospect or a trap, or a localized zone of decompression which can not be identified as a trap because of the small size or some complicating factors. For example, such a region can be represented by a karst zone in the vicinity of the boundary between the blocks with different moments of modern tectonic movements. Therefore, the karst generation processes should develop faster in the decompression region located near the boundary between the blocks than in a region of compression. The contact region between the blocks can be undefined visually on the seismic sections since it has the shape of a narrow vertical zone.

The physical basis for estimating the pressure parameters on seismic data is the direct functional relationship between the total ground pressure in a certain region of the medium and the instantaneous pressure created by a seismic wave propagating in the same region: the wave equation in its fundamental form is formulated via initial (the components of total ground pressure) and instantaneous (dynamic) stresses (the equation of M.Biot). The necessary condition for reflection of a seismic wave is a contrast of elastic and density parameters at the stratigraphic interface. The same parameters determine also the contrast of total ground pressure components at a given interface. Besides, the modern dynamics of a basin cause an extra pressure with respect to the normal gravitational value, and this extra pressure will lead also to a change of the elastic and density properties of the earth. Hence, the idea of estimating the total pressure from the parameters of seismic wave propagation is, in our opinion, more promissing than the idea of estimating some selective properties of the earth simply because the pressure parameters "automatically" integrate the geometry of the interfaces of formation units and their properties such as density, elastic moduli, general porosity (the matrix porosity plus the fracture one), etc. The value of a DFM-based analysis is its capability of finding the parameters of anomalous ground pressure as well as defining the fluid parameters i.e. the permeability and the fluid pressure. We use seismically derived estimates of total pressure for predicting the relative (effective) permeability within the entire investigated medium. We call such concept "dynamic", and the method for predicting the parameters of permeability and pressure as dynamic fluid method (DFM).

The purpose of this work is to investigate possibilities of the DFM application in several geologically complex areas with "old" recovery of oil and gas. The first stage of the work was devoted to investigations of the areas of oil and gas development (the main product is gas) in the Stratton and Boonsville fields. The main purposes of DFM processing and interpretation of seismic data in the mentioned areas were formulated for the first stage of processing and interpretation as follows:

- to construct detailed maps of anomalous ground pressures for the main seismic horizons and for the whole initial information "cube",

- to evaluate the possibilities for detecting local bodies such as a "channel" and "karst" on the pressure anomalies in the maps of these values for certain horizons and in the volume of the data,

- to collate quality and reliability of detection of these bodies with the results of other known interpretation techniques, 
- to collate the anomalies of the ground pressure with the well productivity data and the fluid pressure in the wells, and to evaluate DFM possibilities in direct prediction of the anomalies of fluid pressure in such conditions.

The general strategy of investigations consists of the two main successive processes:

1. Processing of the seismic data represented in the form of a stacked "cube" with the aim of determining the parameters of total ground pressure on the DFM technology.

2. Interpretation of the parameters of total ground pressure in order to detect localized regions of an anomalous pressure caused either by a formation unit such as a channel, bar, or karst area, or by the current anomalous dynamics in the vicinity of the contact of separate blocks of the sedimentary cover, or by other reasons (for example, by long development of a field, and correspondingly, by distortion of the natural stressed condition, which in turn could result in remigration processes).

To a certain extent this strategy does not depend on the traditional approaches to interpretation, and therefore the results may be independently confirmed, supplemented or become alternative to the conclusions which were formulated on the basis of different approaches (statistical, pseudo-acoustical, etc.). In general, comparison of all results of interpretation should lead to more objective understanding of the geological and physical fundamentals of the fluid process nature in the considered conditions. The role of DFM is to connect the whole series of apparently incompatible parameters related to the basin's geology and derived with the aid of different technologies.

DFM helps understand the fluid geology by determining relative spatial estimates of two significant parameters in the fluid geology, i.e. pressure and permeability.

PROCESSING AND INTERPRETATION OF SEISMIC DATA IN DFM TECHNOLOGIES (GENERAL INFormation)

The fundamentals of DFM model of a sedimentary basin are the following:

- each formation unit of a sedimentary basin from the lowest level (layer) to the highest one (basin itself) is a naturally destroyed system or, in other words, a discrete medium where the total permeability is determined as a tensor and depends on the size of separate samples and their ordered multitude (the fracture openness) and on the porosity of the sample material (the rock porosity),

- the "basin - basement" system with a discrete structure is by definition an active medium continuously found in the state of a "viscous flow" as the response to the modern movements of the basement, and in this "basin - basement" system is characterized with the total pressure continuously varying in time (on the geological scale).

- irregular distribution of the total pressure predetermines coordinated variation in time of the fluid pressure, and consequently, a directional fluid flow from the high-pressure regions to low-pressure regions appears in different intervals of a layered system with the discrete structure.

The more detailed information on DFM is given in the Appendix 1 and in the papers listed in this appendix. (see a more detailed explanation on 
1. the concept that the contact of two discrete formation units of any hierarchical level is considered as a plane of break of the medium continuity independently of the contrast of the elastic and density properties of the rocks at the contact;

2. that such a plane of break acts as a seismic reflector according to DFM;

3. that the attributes of the wavelets reflected from this contact depend on the applied total ground pressure (and on the contrast of the elastic moduli at the contact, since the difference in the elastic moduli increases the pressure contrast).

In general case this dependence $\theta$ in $i$-th point of the reflecting interface occurring at the depth $z$ is given by:

$$
\begin{aligned}
& \theta_{i}=f\left(\frac{\delta P_{i}}{\delta z}\right)=\left[\frac{A_{i}}{\widetilde{A}_{i}}\right]^{b}\left[\frac{\widetilde{\omega}_{i}}{\omega_{i}}\right]^{c} \approx \frac{\delta P_{i d}}{\delta z} \frac{1}{k_{i s}} \\
& k_{i s}=s \frac{\delta P_{i}}{\delta \xi_{i}} \\
& P_{i}=P_{i g}+P_{i d}
\end{aligned}
$$

where:

$P_{i}$ is the total ground pressure,

$P_{i j}$ is the pressure caused by the gravity load,

$P_{i d}$ is the anomalous pressure (exceeding the hydrostatic pressure) caused by the modern movements (external loads) and by anomalous fluid pressure,

$k$ is is the coefficient of effective stiffness of a boundary,

$s$ is the scale coefficient depending on the discreteness of the medium from both sides of the boundary (the discreteness is expressed via the volume of one sample normalized by the considered volume of the medium),

$\xi_{i}$ is the value of total deformation of the boundary caused by $P_{i}$,

$A_{i}, \omega_{i}$ are the amplitude and frequency of the reflected wavelet,

$\widetilde{A}_{i}, \widetilde{\omega}_{i}-$ are the amplitude and frequency of the incident wavelet,

$b, c$ are the coefficients taking into account the type of formation units (terrigene or carbonate sediments).

In essence the value $\theta \mathrm{i}$ corresponds to the reflection coefficient for a normally incident elastic body wave and, in contrast to the classical definition, is the function of the frequency of incident and reflected wavelets. Thus, the reflection coefficient of an elastic wave in the model of a discrete irregularly stressed medium is functionally dependent on the anomalous pressure acting in the reflection point. And if we determine the relative variability of the reflection coefficient 
along the reflecting horizon, then this variability will serve as the relative estimate of the anomalous pressure gradient along the same horizon. Further in this paper we will often use the term "anomalous pressure" without mentioning the sense of its spatial and physical definiteness as a gradient, but we will always have this in mind. On the basis of the said considerations, the general idea of seismic data processing with the purpose of estimating the anomalous pressure gradient and its subsequent interpretation lies in the following:

\section{A. Seismic data processing targeted at estimating the anomalous pressures.}

A stacked seismic section obtained as a result of the full cycle of classical processing of CDP gathers using 2D or 3D technologies will serve as the initial information. We will accept that the attributes of the reflection events in this section correspond to the true amplitudes and frequencies of the reflection wavelets. Then we will accept/consider(?) the values $A_{i}, \omega_{i}$ in the formula ( 1 ) as $a_{i}$ and $f_{i}$ of Hilbert transformation of each wavelet of given reflection (the values of instantaneous amplitude and frequency), and we will denote the values $\tilde{A}_{i}, \widetilde{\omega}_{i}$ as $\tilde{a}, \tilde{f}$ and find them as the average values of all $a_{i}$ and $f_{i}$ for the selected horizon. In this case the average estimate $\tilde{a}, \tilde{f}$ is a certain estimate of the incident wavelet in the medium with a constant total pressure $P$ at the depth of given reflecting horizon. We can obtain a more complex estimate of $\tilde{a}, \tilde{f}$ on the strength of horizontal variation of the pressure $P$ along this horizon due to lithological changes in the sequence (variability of the density and the elastic moduli in the overlying sequence, for example the velocity of seismic wave propagation). The general scheme of estimating $\theta_{i}$ for one selected horizon can be as follows (calculations are based on the DYNAMIXt scheme):

1. Picking the same cycle of the wavelet along the reflecting horizon.

2. Determination of the values of instantaneous amplitude and frequency $\left(a_{i}\right.$ and $\left.f_{i}\right)$ in each point $t_{0 i}$.

3. Determination of the average estimates $\tilde{a}, \tilde{f}$ for all values $a_{i}$ and $f_{i}$ for given horizon.

4. Calculation of the values $\theta_{i}$ in each point using the formula ( 1 ).

5. Normalization of the calculated values $\theta_{i}$ in the range between the minimum and maximum values.

It is necessary to realize this scheme for maximum possible number of the reflecting interfaces in given seismic section aiming at subsequent calculation of the integrated estimates $\theta_{i}$ and construction of the vertical maps of these estimates in the plane of a seismic section. The alternative variant for estimating $\theta_{i}$ in the plane of a seismic section is the scheme based on the principle of regular selection of arbitrary number of the horizontal lines with constant values of $T_{0}$ and application of the described scheme for each line with the use of the specified window $T_{0}$ $\pm \Delta T_{0}$ where the values $a_{i}$ and $f_{i}$ are defined as the average values in the window. The obtained estimates $\theta_{i}$ in the plane of given section are the instantaneous estimates of the anomalous pressure gradient, and these estimates can be used for deriving the integrated estimates $\tilde{\theta}_{i}$ by summing the values $\theta_{i}$ in the specified time interval of the seismic section or for several picked horizons. In this case the values $\tilde{\theta}_{i}$ will reflect some generalized estimate of the anomalous pressure gradient, and the latter estimate should reflect the general trend of pressure distribution in the plane of the section. Analysis of such estimate can lead to the conclusions about the 
boundaries of anomalous areas in the context of general modern dynamics of a sedimentary basin.

The second possible scheme for estimating the anomalous pressure can be built in the spectral domain of given time section (the calculation scheme is DYNAMIXs). Here we proceed from the following idea:

$$
S_{j}=S_{j} \theta_{j S}
$$

where

$S_{j}$ is the spectrum of $\mathrm{j}$-th trace of the section (the whole trace or a specified time interval),

$S_{j \theta}$ is the spectrum of reflectivity time function (the function of $\theta i$ values),

$S_{j s}$ is the spectrum of seismic source (the spectrum of incident wavelet).

Since the needed function is the function $\theta_{i}$, then:

$$
S_{j \theta}=\frac{S_{j}}{S_{j s}}
$$

The estimate $S_{j S}$ is the most difficult to derive and the most unstable. We can determine this estimate using the same principles which were used in the first scheme, i.e. as an average value obtained in the specified interval by applying smoothing in different windows to the averaged spectrum. After the optimal spectrum $S_{j \theta}$ is found for each trace, we will proceed again to the time domain and determine the estimate $\theta_{i}$ using the scheme described above. In general, this approach allows for a more detailed analysis of the anomalous pressure estimate, which is leads to detecting local bodies such as channels and karst.

Processing of all initial information «cube» was carried out independently for each vertical section (in in-line or cross-line direction) or for each horizontal section. In the latter case we derived estimates which were slightly different from the estimates in the vertical sections since the average estimate in a horizontal plane will somehow differ from the average estimate in one vertical section in the same time interval. These differences are significant if the geometry of the seismic interfaces is quite complicated within the cube, or there are numerous "pinchouts" of formation units. However, based on our experience in data processing in different basins, the differences in the mentioned technologies are small enough. Taking into account possible weak effects from local bodies, in this work, we tested a variety of techniques estimation of the anomalous pressures.

The anomalous pressure estimates derived from the amplitude and frequency parameters of the reflected signals can be essentially supplemented if we see fulfilled two more considerations:

- an estimate of the total ground pressure includes the gravity component (3) with a magnitude depending on the depth of each considered reflection point. But the formula (1) has been deduced for the variant free from the static influence of the main gravity component (indirectly, via the general influence of pressure on the stiffness coefficient of 
a boundary, the gravity component has already affected the value of the reflection coefficient),

- beside the vertical gradient of the anomalous pressure (which is accounted for in the formula (1)), a horizontal gradient of the anomalous pressure is also observed along the seismic horizon, and the horizontal gradient is caused by the horizon shape as well as by possible variation of lithological and other petrophysical properties of sedimentary rocks within given stratigraphic interval.

Therefore, the derived estimate of the anomalous pressure $\theta \mathrm{i}$ may be supplemented on the basis of the following expression:

$$
\bar{\theta}_{i}=\theta_{i}+e \Delta t_{i}+\left.w \frac{\delta \theta_{i}}{\delta x}\right|_{\max }
$$

The formula (6) has dimensionality of pressure in relative physical units $(P=\rho g h)$ in the case if we take the density $\rho$ of the overlying sequence as unity, and all pressure components are normalized in their own ranges from minimum to maximum values (i.e. in the range $0 \div 1$ ). Each component, except the main one, is added with its own weights e, w. Let's call the obtained estimate as an integrated one since it has been determined with account for the dynamic pressure as well as with additional account for static (structural) components.

The derived sets of the anomalous pressure estimates $\theta_{i}$ or $\overline{\theta_{i}}$ for the «cube» of seismic data are presented in three possible variants:

- a map of the anomalous pressure estimates for the seismic reflecting horizon in the form of a matrix of the values $\theta_{i}$ in the points $X_{i}, Y_{j}, T_{0 k}\left(Z_{k}\right)$. This map will be further called as DFM map,

- a section of the anomalous pressure estimates in the plane of one vertical cross-section of the «cube», or DFM section,

- a multitude of the functions $\theta \mathrm{i}$ ( $\mathrm{t}$ or $\mathrm{z}$ ) in the format of seismic traces, or «DFM cube».

\section{Interpretation of the anomalous pressure estimates.}

The derived set of the anomalous pressure estimates for all the cube or for its vertical or horizontal sections and selected horizons (DFM sections and maps) may represent a rather specific basis for interpretation which can be divided into two stages, i.e. a qualitative interpretation and a quantitative interpretation.

The qualitative interpretation is similar to the analysis of the displayed seismic traces in traditional technologies, but in this case it is necessary to account for some features of the anomalous pressure distribution from the ground of geodynamics and the mechanics of the elastic media. Here, we mean the following general circumstances:

- a localized anomaly of the pressure estimate in given seismic horizon may be a consequence of the spatial influence of a local body (sand channel, karst, etc.) with the elastic properties that are different from those of the background medium. This local body can be actually located below the given horizon or above it, 
- the same circumstance is true for the areas of "old" durably developed oil or gas fields where complex but regular contours of the pressure anomalies may be observed above and below the developed reservoir, because the development processes strongly influenced on the distribution of natural pressures (which existed in the earth before beginning of the field development),

- within an active block of the sedimentary basin, a common bias toward increased or decreased pressures (compression or decompression blocks) should be observed for all reflecting horizons from top to bottom, and this common indicator may be used for determining outlines of a block characterized with certain dynamics,

- lines of tectonic faults or block boundaries should be reflected in plane and cross-section in the form of corresponding linear zones of anomalous pressures. And anomalies of different signs with a noticeable linear gradient zone between them may be observed.

To jointly evaluate the geometry of a reflecting or stratigraphic horizon and the anomalous pressures, the corresponding isochron map or depth map is superimposed on the DFM map. In this case the anomalous pressure estimates are presented using a color palette, while the contours are presented in the vector form.

Certainly, the main result of DFM processing and interpretation includes the outlines of the regions of low total pressure, since the fluid flow will be directed into these regions if all other circumstances are favorable, and fluid accumulation and retaining is most probable in such regions. Here we should fix the sign and general physical perception of the anomalous pressure:

- a decompression region has the positive sign in the components of stress,

- high fluid pressure has the same positive sign and high absolute value,

- in the geological time scale at slow fluid flow and low speed of deformation ("flow") of the solid medium the total anomalous pressure may be considered as a positive sum of anomalous ground and fluid pressures, and further we can consider them as interconnected values.

In that way we will further suppose that the value $\theta_{i}$ is always positive, and its large numerical magnitude we interpret as a decompression region from the ground of solid phase, and as a high fluid pressure from the ground of liquid phase.

Quantitative interpretation of the results of anomalous pressure estimation includes prediction of fluid flow parameters within the cube. We will further accept that the derived relative estimate of the total anomalous pressure can be calibrated and recalculated with the aid of borehole data (initial or current formation pressure) into the true anomalous pressure $P_{d}=u \theta_{i}$, where $u$ is the transfer coefficient. In this case the speed and direction of the fluid flow $\vec{V}$ will be determined by the Darcy's equation in the following form:

$$
\vec{V}=-\frac{c}{\mu} \nabla P_{d}
$$

Here $\tilde{n}$ is the permeability of the medium in given point, and $\mu$ is the viscosity of a fluid.

Now we will consider the case where the sources and drains of a fluid are generated in each unit volume of the discrete medium due to variation of the total voidness as a consequence of 
continuous change of the total ground pressure toward its increase (compression) or decrease (decompression). Then from the continuity equation we have

$$
\frac{\bar{o}}{\bar{o} t}(F r)+\nabla(r V)=0
$$

for an incompressible fluid with the constant density $\rho$ it follows that

$$
\nabla V=-\frac{\bar{o} F}{\bar{o} t}=-q
$$

where $V$ is the speed of Darcy flow, $F$ is the total voidness (the fracture and porosity space), and $q$ is the speed of its variation $\left(\tilde{\mathrm{n}}^{-1}\right)$. We will also accept that the speed of volumetric deformation $\Delta D / \Delta t$ of some volume of the discrete medium $D$ is related only to the speed of change of the total voidness:

$$
q=\frac{\Delta D}{D \Delta t}
$$

By substituting the equation for the speed of Darcy's flow $V=c / \mu$ grad $P_{d}$ into the formula (3), we will obtain the Poisson's equation for pressure in the following form:

$$
\nabla^{2} P_{d}=-\frac{\mu}{c} \nabla \frac{c}{\mu} \nabla P_{d}+\frac{\mu}{c} q
$$

In the case of a medium with homogeneous permeability $c_{0}$ and a homogeneous fluid with the viscosity $\mu_{0}$ we have:

$$
\Delta P_{d}=-\frac{\mu_{0} q}{c_{0}} \quad \text { and } \quad P_{0}=-\frac{\mu_{0}}{4 \pi c_{0}} \int_{D_{0}} \frac{q d D_{0}}{R}
$$

where $d D_{0}=d x_{0} d y_{0} d z_{0}$ is the deformed volume, and $R^{2}=\left(x-x_{0}\right)^{2}+\left(y-y_{0}\right)^{2}+\left(z-z_{0}\right)^{2}$.

The formula for pressure in the presence of a heterogeneous region $D$, in which $c$ and $\mu$ are the functions of coordinates, can be written in the form:

$$
\begin{aligned}
P_{d} & =P_{0}-\frac{1}{4 \pi} \int_{D}\left(\frac{c \mu_{0}}{c_{0} \mu}-1\right) \nabla_{D} P_{d} \frac{\mathbf{r}}{r^{3}} d D \\
\mathbf{r} & =(x-\xi) \mathbf{i}+(y-\eta) \mathbf{j}+(z-\zeta) \mathbf{k}, d D=d \xi d \eta d \zeta
\end{aligned}
$$

where $\boldsymbol{Ð}_{0}$ may in particular correspond to the equation ( 12 ).

The formulae ( 12 ) and ( 13 ) can be directly generalized for the case of several deformed and heterogeneous volumes, and the values $D$ and $D_{0}$ can coincide partially or completely. Let's suppose that a small deformation does not cause an essential displacement of the permeability boundaries in the considered volumes, that there are several deformed volumes $D_{0}=\Sigma D_{0 j}$, 
$j=1,2,3 \ldots, n$, and that each volume $D_{0 j}$ is characterized with its own speed of volumetric deformation $q_{j}$ and of different signs in the directions of compression and strain of the volumes. Just so the sedimentary cover can be modeled, in the form of a discrete dynamic system. In this case, the problem of basin's dynamics corresponds directly with the problem of the medium permeability variation, and in the form of time function. This finally leads to generation of a natural "communicating" block patterns, i.e. one system of blocks squeezes out the fluid, while the other system of blocks absorbs it.

For regions with sufficient extent along the axis OY comparatively to the dimensions along the other axes, we will rewrite the equation for two-dimensional case (by analogy with the equations (12) and (13)) in the following form:

$$
P_{d}=\frac{\mu_{0}}{2 \pi c_{0}} \int_{S_{0}} q \ln R d S_{0}-\frac{1}{2 \pi} \int_{S}\left(\frac{c \mu_{0}}{c_{0} \mu}-1\right) \nabla_{D} P_{d} \frac{\mathbf{r}}{r^{2}} d S
$$

Using the equation ( 14 ) we can deduce an integral equation for the gradient of potential for the inner points of the section $\mathrm{S}$ :

$$
\nabla_{A} P_{d}=\frac{\mu_{0}}{2 \pi c_{0}} \int_{S_{0}} q \frac{\mathbf{R}}{R^{2}} d S_{0}-\frac{1}{2 \pi} \nabla_{A} \int_{S}\left(\frac{c \mu_{0}}{c_{0} \mu}-1\right) \nabla_{M} P_{d} \frac{\mathbf{r}}{r^{2}} d S
$$

where $\nabla_{A}=\mathbf{i} \partial / \partial x+\mathbf{k} \partial / \partial \mathrm{z}, \quad \nabla_{D}=\mathbf{i} \partial / \partial \zeta+\mathbf{k} \partial / \partial \zeta, d S=d \xi \partial \zeta, \mathbf{R}=\left(x-x_{0}\right) \mathbf{i}+\left(z-\mathbf{z}_{0}\right) \mathbf{k}, \quad \mathbf{r}=(x-$ $\xi) \mathbf{i}+(z-\zeta) \mathbf{k}, d S_{0}=d x_{0} d z_{0}$.

When the integral equation ( 15 ) is solved, we will derive pressure from the equation ( 14. ) using the same matrix of inner values $\nabla_{D} P_{d}$.

Now we will consider a thin horizontal layer placed in the medium which is impermeable for fluids, and also we will unite the volumes of deformation and heterogeneity $\mathrm{S}$ and $\mathrm{S}_{0}$. In this case the equations (14) and (15) shall be written in the form:

$$
\begin{gathered}
P_{d}=\frac{1}{2 \pi} \int_{S} \frac{\mu_{0}}{c_{0}} q \ln R d S-\frac{1}{2 \pi} \int_{S}\left(\frac{c \mu_{0}}{c_{0} \mu}-1\right) \nabla_{M} P_{d} \frac{\mathbf{r}}{r^{2}} d S \\
\nabla_{A} P d=\frac{1}{2 \pi} \int_{S} \frac{\mu_{0}}{c_{0}} q \frac{\mathbf{r}}{r^{2}} d S-\frac{1}{2 \pi} \nabla_{A} \int_{S}\left(\frac{c \mu_{0}}{c_{0} \mu}-1\right) \nabla_{M} P_{d} \frac{\mathbf{r}}{r^{2}} d S \\
\nabla_{A}=\mathbf{i} \partial / \partial x+\mathbf{j} \partial / \partial \mathrm{y}, \nabla_{M}=\mathbf{i} \partial / \partial \zeta+\mathbf{j} \partial / \partial \eta, d S=d \xi \partial \eta, \mathbf{r}=(x-\xi) \mathbf{i}+(y-\eta) \mathbf{k}
\end{gathered}
$$

After scalarization the vector integral equation (17) can be considered as a system of integral equations of Fredholm of the second type if the distribution $c / \mu$ is known and we need to determine the pressure gradient, or as the equation of Fredholm of the first type in the case of unknown distribution of $c / \mu$ if we know the pressure gradient distribution. If our target objective is estimation of the correspondence between pressure and permeability, then it is logical to solve 
the forward problem of determining the pressure gradient on the specified distribution of permeability using formula ( 17 ) as the equation of Fredholm of the second type, and then to calculate pressure using the formula (16).

Thus, as a result of quantitative interpretation we can proceed from the relative estimates of the anomalous pressure derived exclusively from the seismic data, to quite valid final product, i.e. the permeability and the true fluid pressure. For oil engineers this can represent a very useful basis for defining more accurately the resources of the product and for correcting the plan of its recovery. There is no ground to carry out the quantitative interpretation at given stage of investigations, since additional information extracted from all interpretation technologies is required. The next stage will include calculations with the use of the described algorithm and with account for all circumstances revealed at the first stage. As a result the permeability maps will be constructed, and the outlines of actual fluid saturation will be drawn.

It should be noted that all discussed approaches to processing and interpretation of seismic data with the use of DFM technologies have been published and patented (the USA patents from 1997 and 1999). These approaches are described in general in the Appendices 1 and 2 of this report and were tested in different areas within the basins of Western Siberia, Caspian sea, China, Vietnam, Baltic sea, North sea, etc.

\section{PROCESSING AND INTERPRETATION OF 3D SEISMIC DATA IN THE AREA OF STRATTON FIELD}

Stratton field is located within the Kleberg and Nueces counties in South Texas and is related to the type of fluvial sediments of the stratigraphic interval of Oligocene Frio Formation (Bob A. Hardage, Raumond A. Levey, Virginia Pendleton, James Simmons, and Rick Edson,1994: A 3-D seismic case history evaluating fluvially deposited thin-bed reservoirs in a gas-producing property, Geophysics, vol.59, NO.11, p.1650 - 1665, 22 FIGS.). The considered formation FR-4 is related to Vicksburg Fault Zone where the sand-shale sequence is a gas-bearing reservoir.

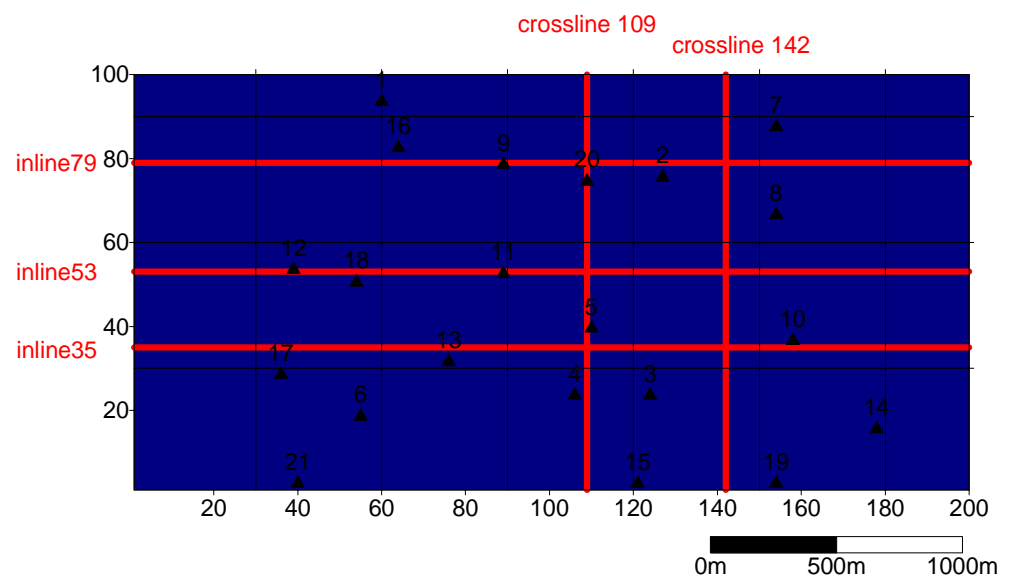

Fig.1. Map of the well locations and cross sections (in-lines 3, cross-lines 2, - red color lines)
The initial seismic data are represented by a seismic "cube" consisting of 100 in-line stacked sections after migration (Fig.1). Several in-lines and cross-lines which were used for estimating the anomalous pressure in the vertical sections are marked by red lines in this figure. Also shown here are the exploration and development wells.

The most consistent reflection interfaces (SH1, SH 2, SH 3, SH 4, and SH 5, see Fig.2) were selected as the main seismic horizons for predicting the anomalous pressures with the aid of the DFM technology. Also shown here are the stratigraphic interfaces delineated with the aid of the borehole data (B 46 - F 39). Thus, the selected seismic horizons completely represent the whole producing interval of fluvial sediments of the producing formation within the interval of $1.05-1.75 \mathrm{~s}$. With account for locations of the mentioned seismic and stratigraphic interfaces we have divided the whole producing interval into 
three intervals (IA, IB, and IC) also shown in the same figure. The choice of such intervals is

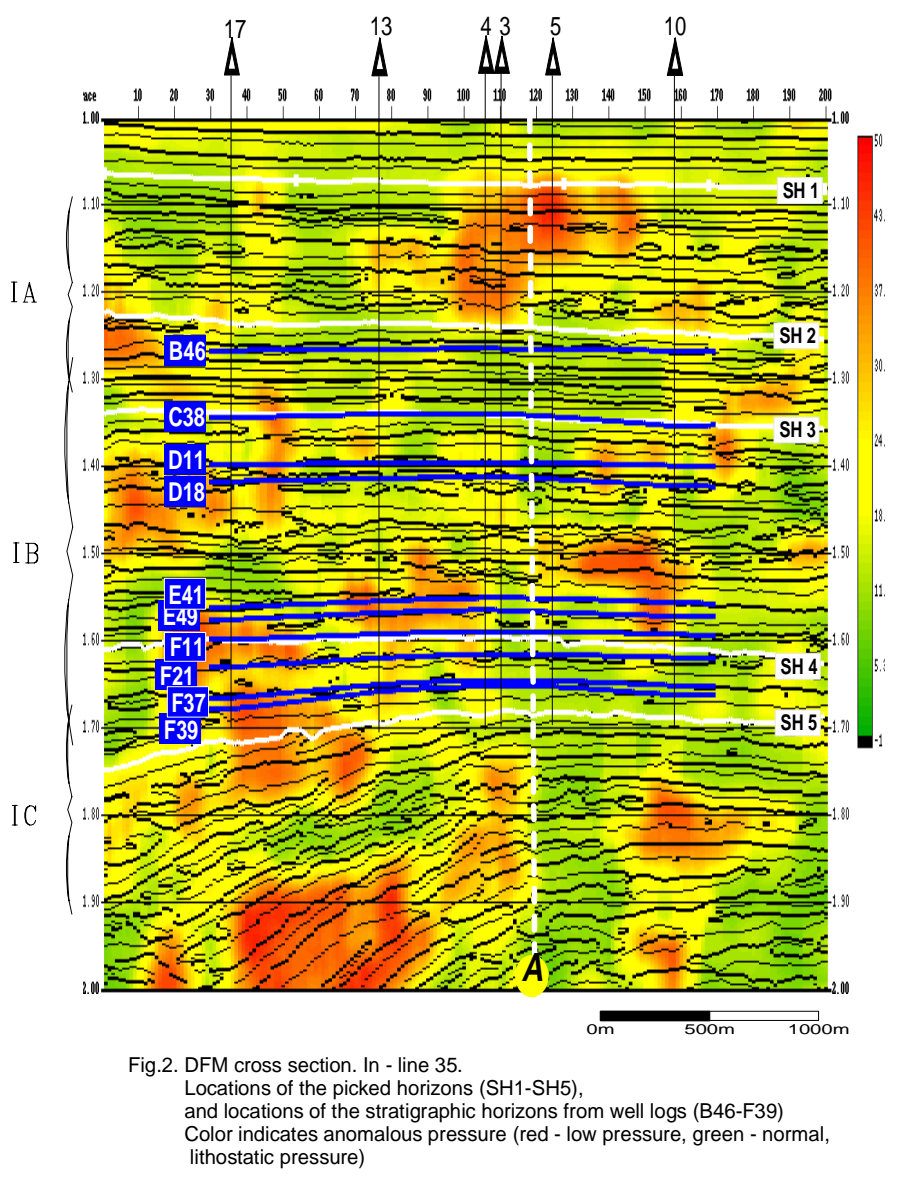
dictated by subsequent application of the DFM technology. A DFM section shown in given figure as the physical basis represents the anomalous pressures estimate (color) and the singular points of the phase characteristic of each seismic trace. These singular points indicate the values of $+/-180$ degrees and zero-crossings (larger black dots correspond to the 180-degree values, while smaller black dots correspond to zero-crossings). This mode is the most convenient and informative one for displaying a DFM section with the purpose of tying the pressure anomalies to corresponding seismic horizons. The color palette for the anomalous pressures is always the same for vertical sections and corresponds to the following principle: the red color indicates a relative decrease of the total pressure gradient (a decompression region), the green color corresponds to the values of normal lithostatic gradient of the total pressure.

\section{À. Processing of the initial «cube» according to DFM technology.}

The general scheme of data processing in given area includes the following logical stages:

1. Calculation of the anomalous pressures in several time intervals.

Figs.3, 4, and 5 show the DFM maps for the time intervals IA, IB, and IC correspondingly. In this case the anomalous pressures were estimated in the time domain with the aid of DYNAMIXT scheme described in the previous section. The values $\theta_{i}$ were calculated for horizontal slices of the cube with the interval of $4 \mathrm{~ms}$ TWT and the vertical averaging window of 5 samples $(-4 \mathrm{~ms}+4 \mathrm{~ms})$. All the matrices of the calculated values $\theta_{i}$ were summed in each time interval, and in that way one DFM map was produced for each time interval. These DFM maps are related to the centers of corresponding time intervals and represent generalized estimates of the anomalous pressures for the whole volumes of selected time intervals. The same color palette is used to indicate the anomalous pressure estimates in each map: the red color corresponds to low-pressure regions (decompression zones), while the blue color corresponds to regions of normal or increased loads (compression zones). Besides, the isochron map for one of the seismic horizons located in given interval is drawn on each DFM map (fig.3 - SH1, fig.4 $\mathrm{SH} 3$, fig.5 - SH4). In this case the pattern of time contours aids in understanding of the correspondence between the pressure anomalies and the general geometry of the layered structure in each time interval. 


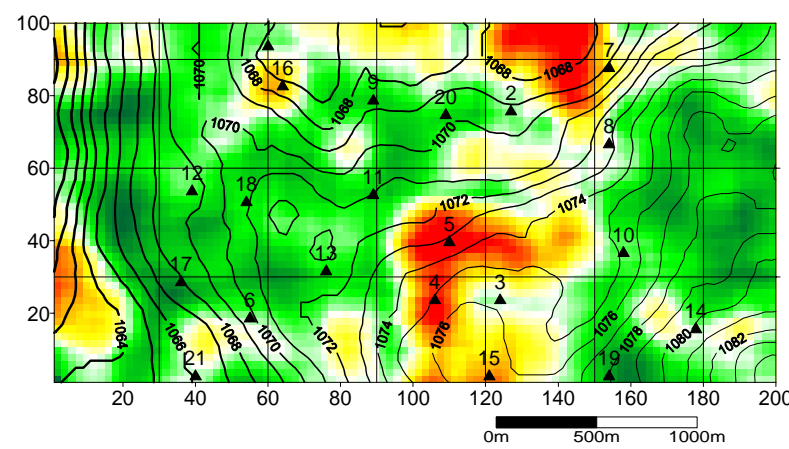

Fig.3. Map of anomalous pressure for the time section IA (interval A - 1100 - $1300 \mathrm{msec}$.) and SH1 isochrons (black lines)

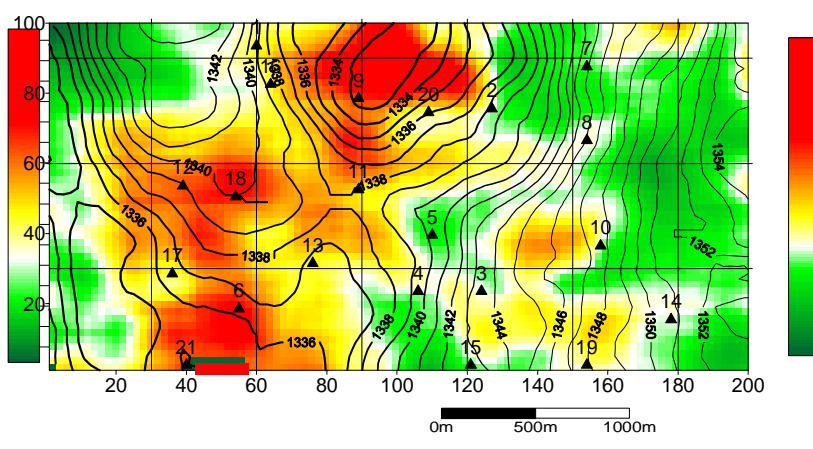

Fig 4. Map of anomalous pressures for the time section IB (interval B $1300-1700 \mathrm{msec}$ ) and SH3 isochrons (black lines)

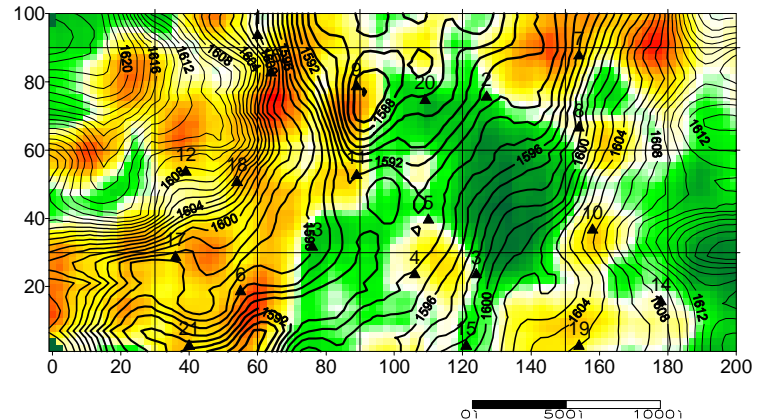

Fig 5. Map of anomalous pressures for the time section IC (interval C 1700 - $1900 \mathrm{msec}$ ) and SH4 isochrons (black lines)

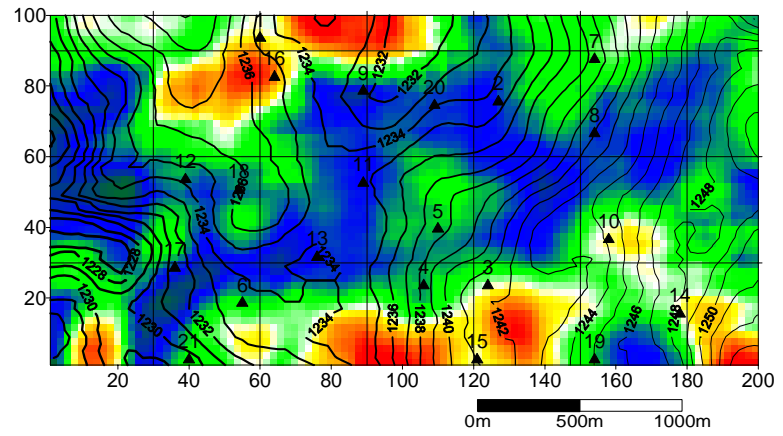

Fig 7. Map of anomalous pressures (colors) \& isochrons (black lines) along the horizon $\mathrm{SH} 2$

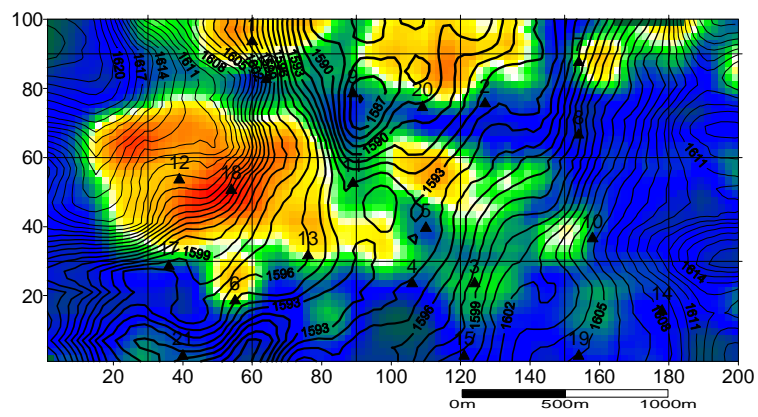

Fig.9. Map of anomalous pressures (colors) \& isochrons (black lines) Horizon SH4.

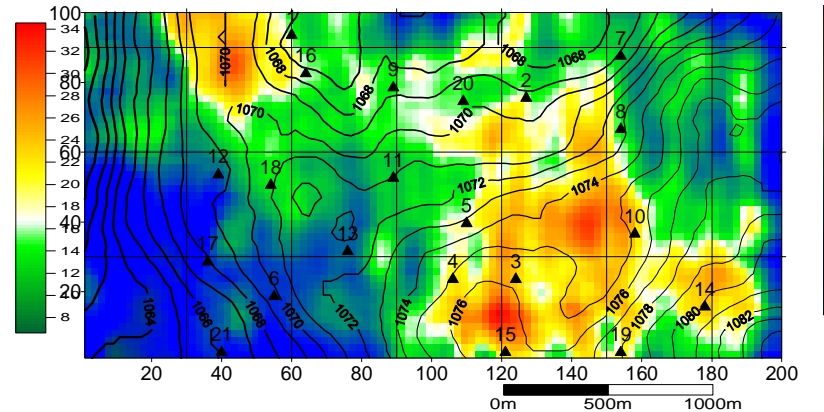

Fig.6. Map of anomalous pressures (colors) \& isochrons (black lines) along the horizon $\mathrm{SH} 1$

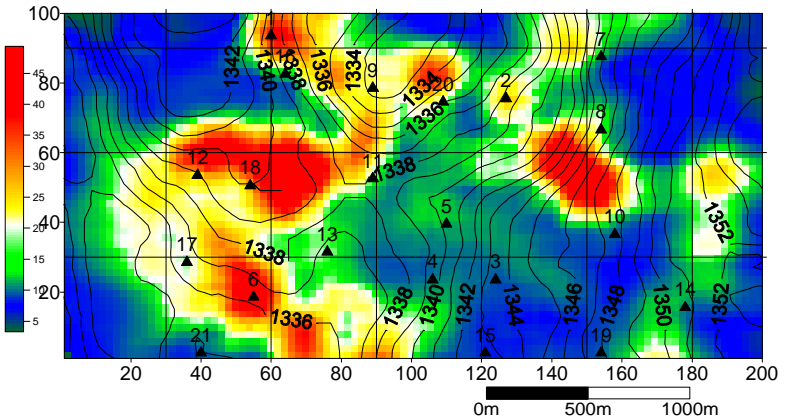

Fig.8. Map of anomalous pressures (colors) \& isochrons (black lines) along the horizon $\mathrm{SH} 3$.

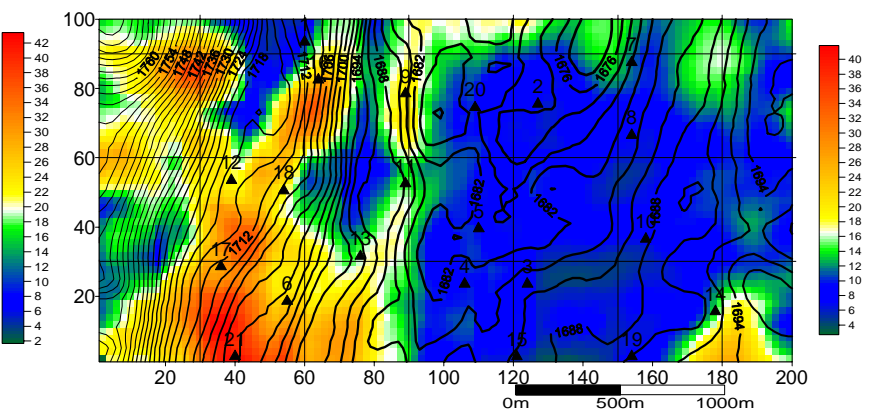
Fig.10. Map of anomalous pressures (colors) \& isochrons (black lines)
along the horizon SH5.
Final Technical Report

October, 2002
Michigan Technological University

DE-AC26-98BC15135

Page 147 
2. Estimation of anomalous pressures on the reflecting horizons.

Figs.6, 7, 8, 9, and 10 show the maps of anomalous pressure estimates calculated with the aid of the main scheme (DYNAMIXT) for each selected and manually tracked seismic horizon (SH1, $\mathrm{SH} 2, \mathrm{SH} 3, \mathrm{SH} 4, \mathrm{SH} 5)$. The same color palette is used in these maps to indicate the anomalous pressure estimates. The isochron maps for corresponding horizons (the result of horizon tracking in the coordinates of the initial cube) are drawn on the DFM maps.

3. Calculations of the horizontal gradients of anomalous pressure.

Fig. 11 and 12 show the maps of the horizontal gradients for several seismic horizons (SH2, SH3, and SH4) and the sum of the derived gradients for these horizons. The horizontal gradient was determined with the aid of the following principle. Maximum of the gradients calculated in each point of the DFM map for the rays passing through given point in each 45 degrees was selected. The gradient was determined on the basis of 100-meter interval (about one dominant wavelength). The gradient values are indicated in the figures with the aid of color (the red color corresponds to high gradients, and the blue color corresponds to low gradients).

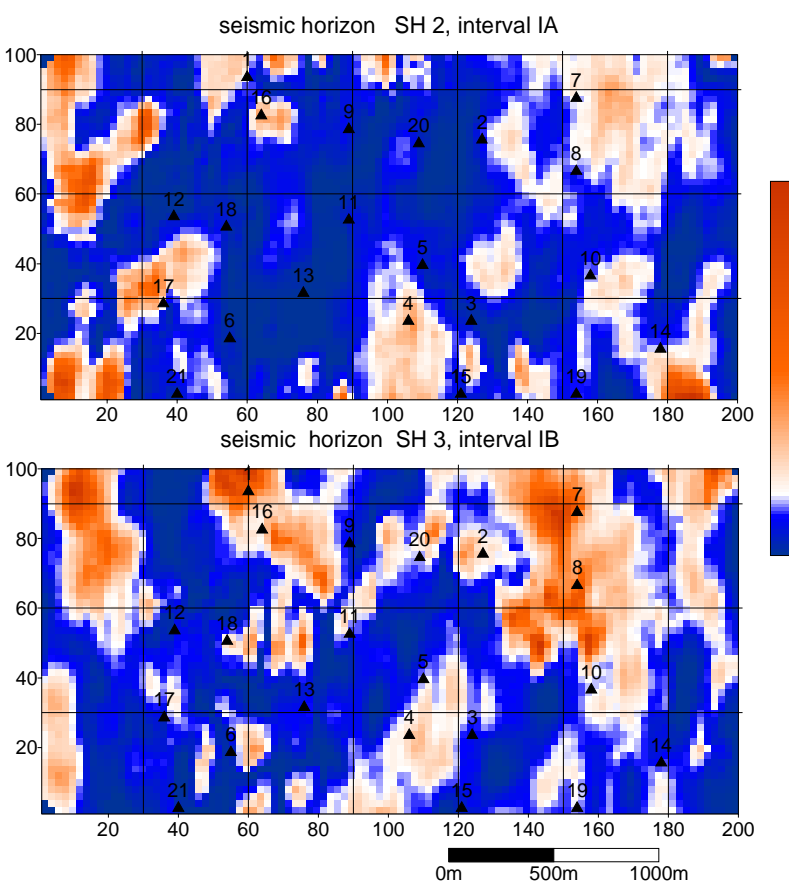

Fig.11. Maps of integrated pressure gradients on intervals IA and IB

4. The structural DFM maps.

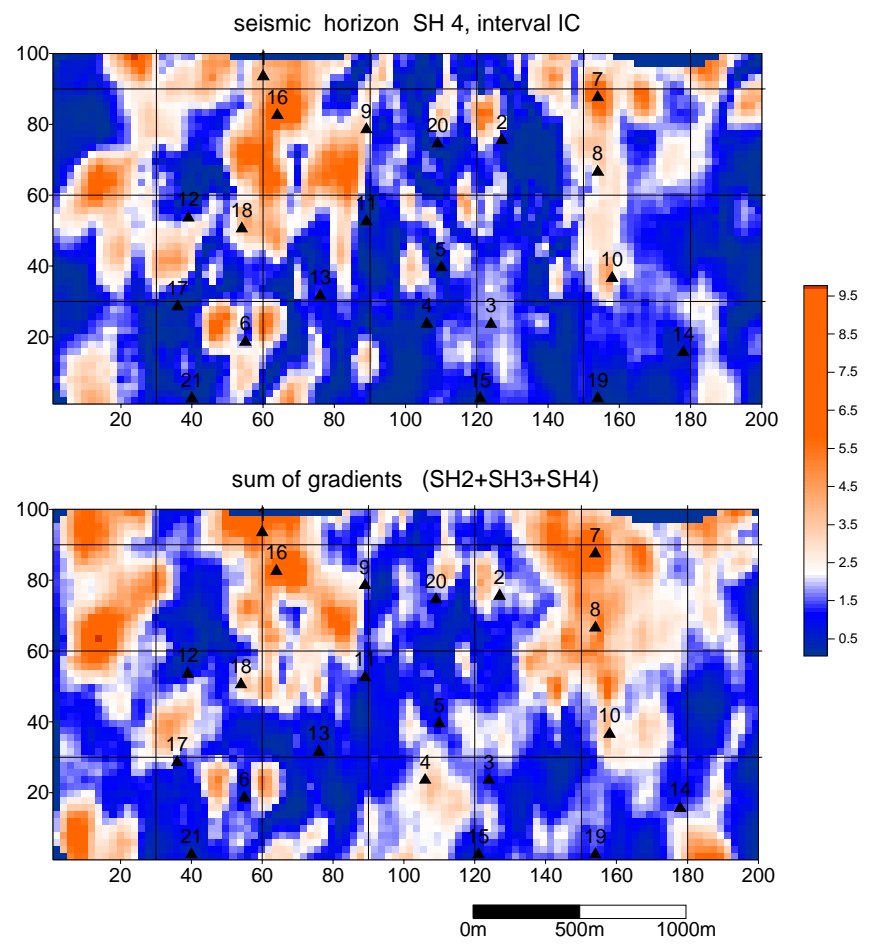

Fig.12. Map of integrated pressure gradients in the IC interval (upper) and map of integrated pressure gradients in the 3 intervals : IA+IB+IC Stacked pressure gradients (lower)

Fig. 13 shows the structural DFM maps for three stratigraphic intervals: b46, c38, f11. The choice of these stratigraphic intervals is explained by the proximity of the tracked seismic horizons: b46 to SH2, c38 to SH3, and f11 to SH4. The maps are obtained by superimposition of the anomalous pressure maps for the mentioned seismic horizons (these map are shown separately in figs. $7,8,9)$ on the depth maps for the stratigraphic horizons. These maps will serve as the physical and geological basis for estimating the parameters of fluid flows at the stage of subsequent quantitative interpretation. 

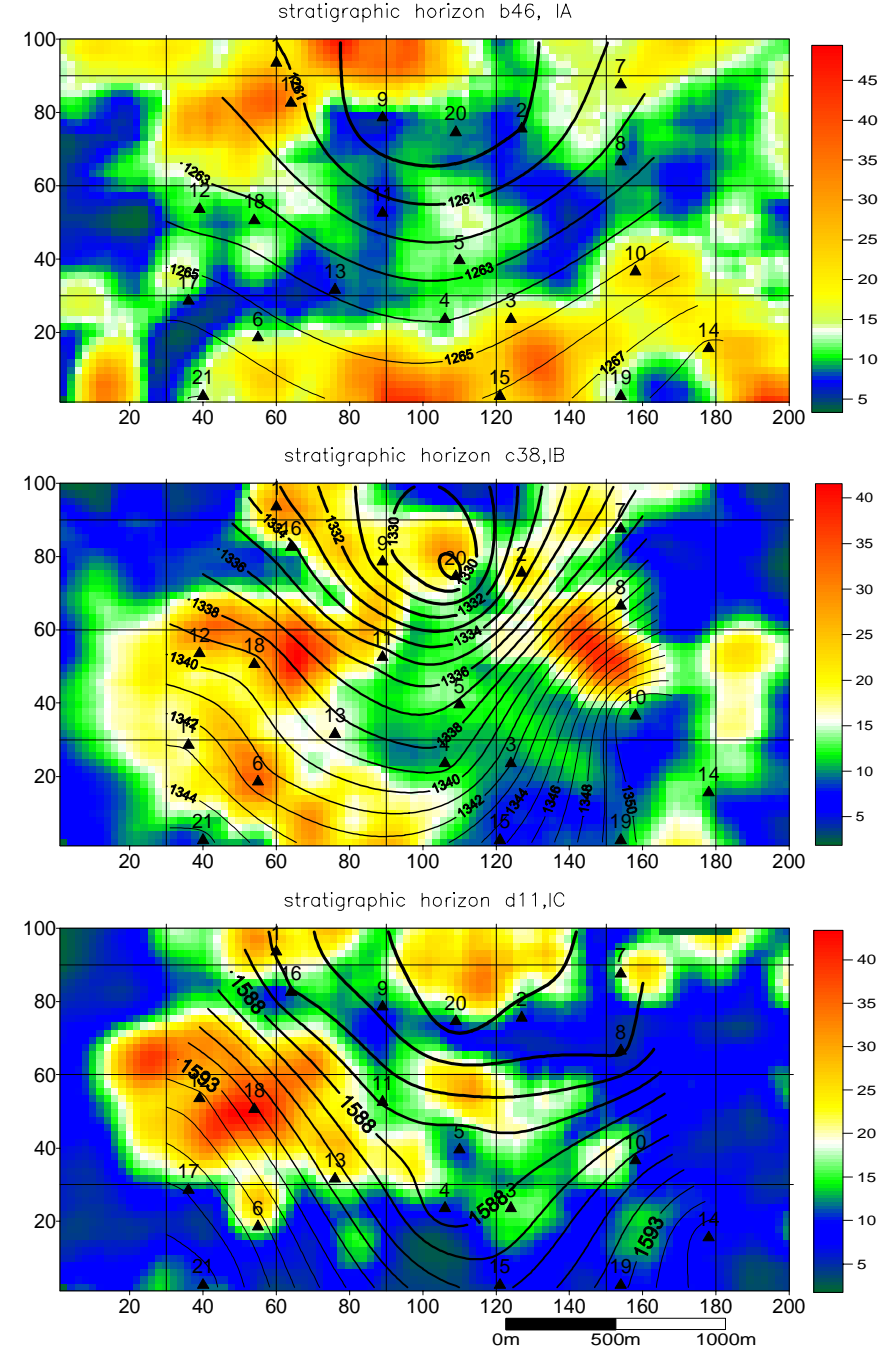

Fig.13. Map of pressure anomalies for interval IA,IB,IC overlaid on structural gelogic maps respectively b46,c38,d11

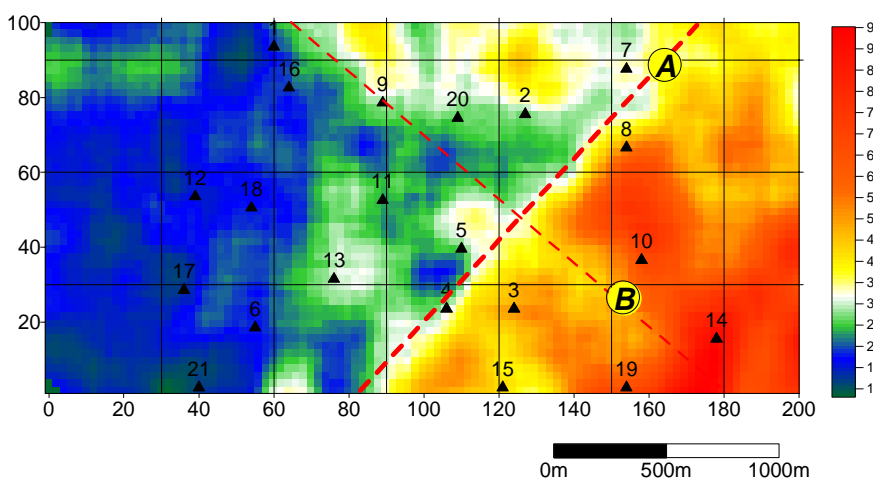

Fig.14. Map of pressure anomalies on "deep section" interval (2200 - $2700 \mathrm{msec})$
5. Estimation of the anomalous pressures in the sequences underlying the producing intervals.

Such estimation is needed to clarify general elements of the dynamics of the fluvial basin's base. As a matter of fact there should be some general scheme of the dynamics of the basin's base to determine river flows in the periods of generation of sand bodies such as «channels». Most likely, the boundaries of active blocks should serve as such general dynamic distorting elements, and corresponding local highs and lows on the ground surface (horizontal gradients of the surface relief) appear along these boundaries in each stratigraphic interval in the period of its generation. In this case we have selected a time interval of $2.200-2.700 \mathrm{~s}$, and the DFM map has been produced for this interval according to a scheme similar to that applied for calculating the interval estimates of the anomalous pressures in the overlying intervals (IA,IB,IC). This map shown in Fig.14 completely justifies our hopes to determine the boundaries of active blocks. The outlines of the anomalous pressure regions have strictly rectangular (block) shape thus indirectly testifying to the modern dynamic conditions of the basin on the one hand, and to its connection with the dynamics during earlier periods, comprising the time of generation of the fluvial basin, on the other hand. 
6. Calculation of the anomalous pressure estimates in the vertical sections.

Figs.15,16,17,18 show the DFM sections produced with the aid of DYNAMIXt scheme in vertical sections of «cube» for selected directions (correspondingly, in-line sections 79,53,142 and cross-line section 109 ).
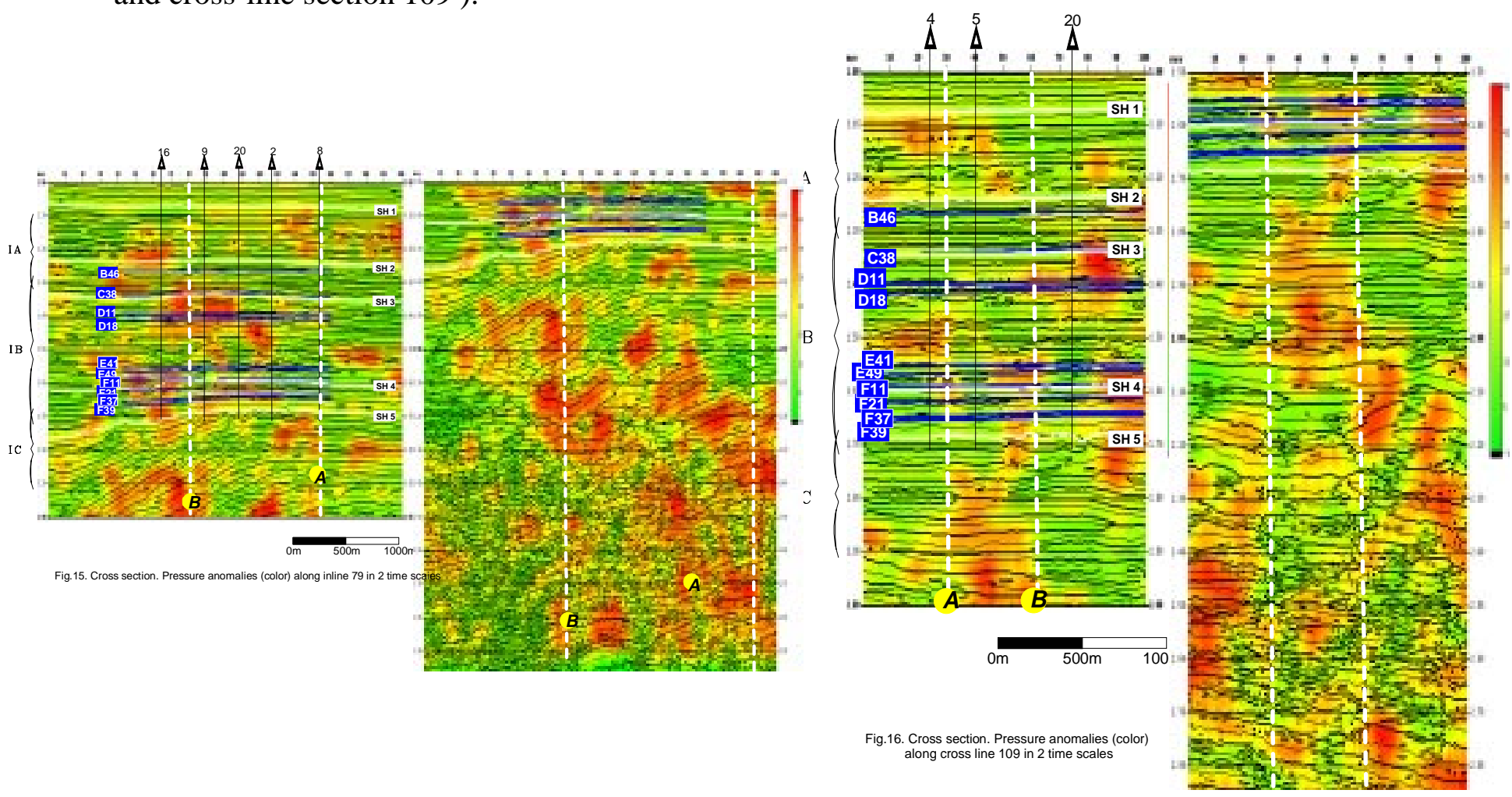

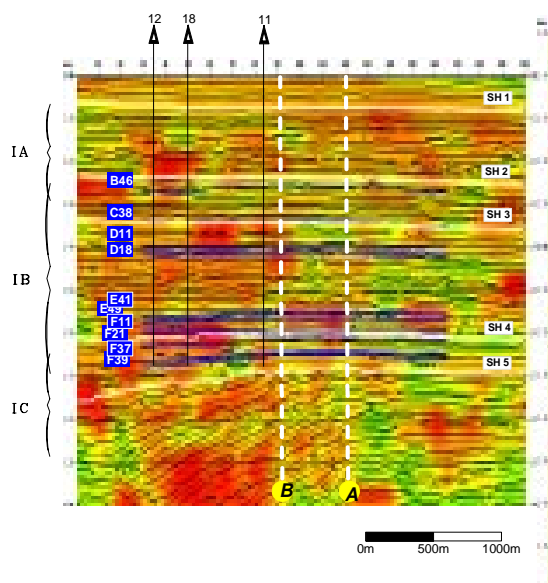

Fig. 17. Cross section. Pressure anomalies (color)
along in line 53 in 2 time scales
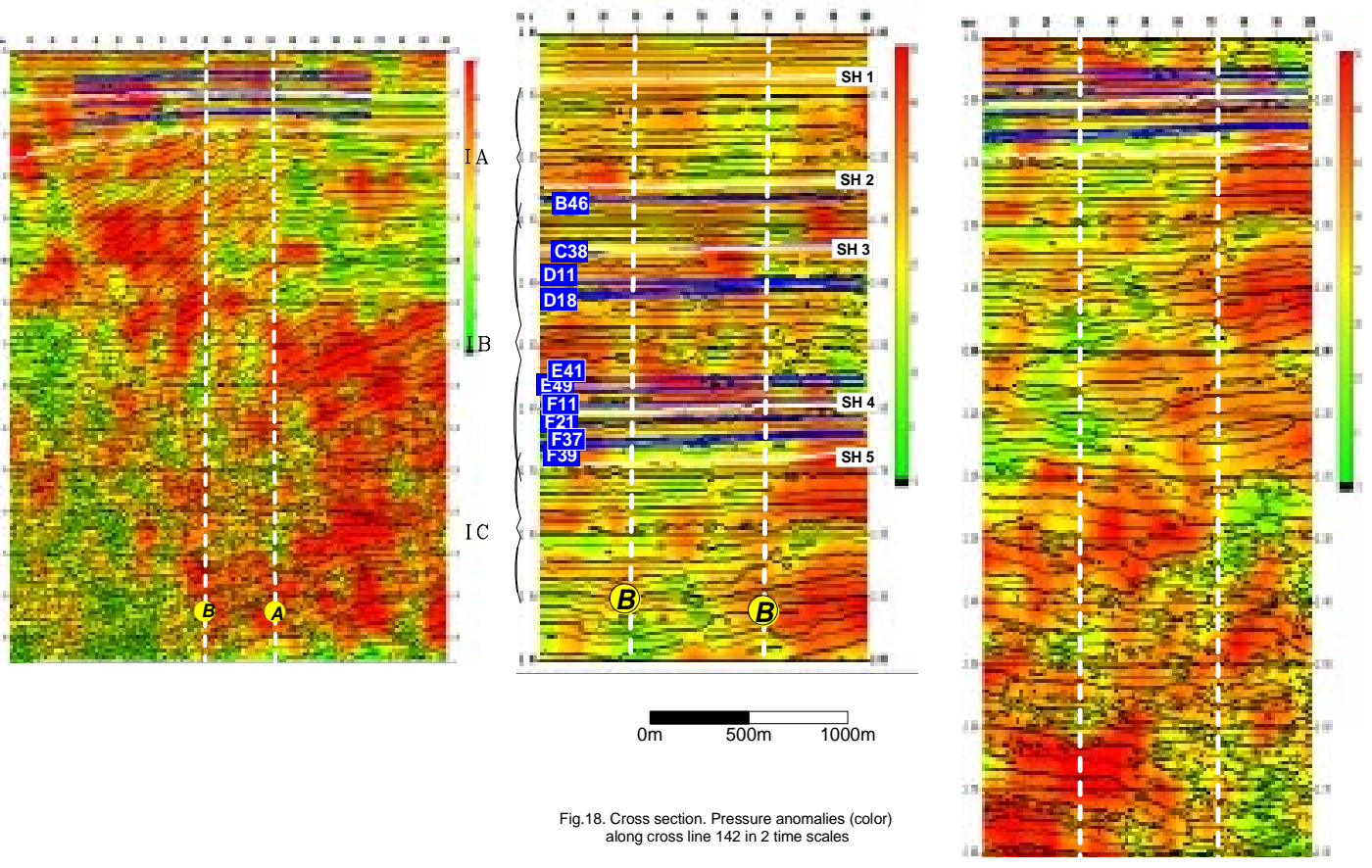

Final Technical Report

October, 2002
Michigan Technological University

DE-AC26-98BC15135

Page 150 
7. Calculation of the anomalous pressure estimates in the whole volume of the «cube».

Calculations of the anomalous pressure estimates in the whole volume of the «cube» were carried out with the aid of DYNAMIXt scheme in two variants:

a) for horizontal slices with interval of $\Delta t_{0}=4 \mathrm{~ms}$ in the range from $t_{o}=0$ to $t_{o}=2.5 \mathrm{~s}$,

b)for vertical sections of the cube for all in-lines in the same range of TWT $\left(t_{o}\right)$.

It is obvious that these two variants may have differences in the levels of anomalous estimates because of the differences in estimation of the average values in horizontal and vertical planes for the same TWT $\left(t_{o}\right)$. The format of the pressure values corresponds to the initial format and the structure of the cube (SEG Y), thus giving opportunities for analysis of spatial regularities of the estimates of the anomalous pressures with the aid of any known seismic technologies such as GeoQuest, Stratimagic, etc. The calculated «cubes» of the pressure estimates were transmitted to MTU for analysis.

Interpretation of the anomalous pressure.

All the mentioned results of processing were used at the stage of qualitative analysis which was carried out in the following interconnected directions:

- estimation of the general scheme of dynamics of a fluvial sequence,

- delineation of local anomalous stratigraphic elements such as «channels» as the main producing bodies,

- preliminary evaluation of the productivity of the studied stratigraphic interval,

- determination of the initial conditions for the stage of quantitative interpretation which includes calculations of the parameters of fluid flow (permeability and fluid pressure).

Now we will bring the main results of the stage of qualitative interpretation in this sequence and will formulate a number of recommendations for the final stage of interpretation and practical usage.

The general scheme of dynamics of a fluvial sequence.

Development of the scheme of dynamics of the sedimentary cover is mainly targeted at detection of the boundaries of blocks into which all the sedimentary cover or its sufficiently large interval can be divided with a certain degree of probability. Development of this scheme is based on the following general principles of geodynamics of discrete media:

- the outlines of the blocks should be mainly rectangular,

- the sign or magnitude of the anomalous pressures is different from both sides of the boundaries of dynamically active blocks, and their outlines on the DFM maps should coincide with the zones of contrast horizontal gradients of the anomalous pressures,

- since the modern movements of the blocks have a vertical direction, then the boundaries of active blocks should mainly have the vertical dip on the DFM sections and serve as natural separating planes between the regions with different levels and structure of the anomalous pressures, 
- the transverse size of the blocks can not be smaller than the thickness of a formation sequence (in given case the thickness of all fluvial formation sequence is equal to about 1000-1500 m. Therefore, the transverse size of the blocks should not be smaller than the latter value).

According to these principles, the block scheme in the form of a system of lines marked as A, B,

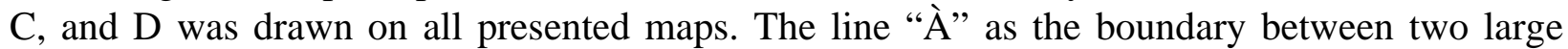
blocks is reliably detected on the DFM map complied for a deep interval below the producing formation (fig.14). This map shows a clear image of a rectilinear boundary between two regions with principally different estimates of the anomalous pressures. This may indicate either the boundary between two different structural and lithological sequences, or the boundary of a regional fault dividing the base of the fluvial basin into two blocks with different dynamics. The same boundary is clearly visible on all the in-line DFM sections (Figs. 2, 15, 16, 17, 18). The lines $\mathrm{B}, \mathrm{C}$, and $\mathrm{D}$ were detected by analyzing the maps of the horizontal gradients of the anomalous pressure estimates (Figs.11, 12) and DFM sections (Figs. 15, 16, 17, 18). All the system of lines (or the boundaries of dynamically active blocks) does not contradict to all other results of processing, i.e. to the DFM maps for time intervals and for reflecting horizons. In all these maps the local regions of anomalous pressures are located beside the detected boundaries or related to the crossings (nodes) of the block boundaries, thus increasing fidelity and reliability of the constructed scheme as a whole.

\section{Local bodies in the fluvial sequence.}

A contrast local region of the anomalous pressure estimates emerges in the DFM map for the reflecting horizon $\mathrm{SH} 2$. The meandering boundary of blue color indicates a compression region similar to the image of a channel in the plan view. In the map for more deep horizon SH3 we observe a decompression region of the same pattern but with a certain shift in the plan view. According to the general principle of anomalous pressure distribution in the vicinity of a local solid body, as a sand channel should be, we can assume with a sufficiently high probability that a channel is located between the two mentioned horizons, and this channel creates a peculiar shadow in the anomalous pressure maps for overlying and underlying seismic horizons. Besides, the meandering pattern of this body is logically explained by the boundaries of delineated blocks (the lines A, B, C, D), i.e. all bends of the channel coincide with the crossings of corresponding boundaries of the blocks. Just so the direction of water flows should be generated with account for the relief of the active bottom of the basin in the period of its continental development and in the conditions of a shallow sea.

\section{Preliminary conclusions on the productivity of the studied reservoir.}

At given stage of interpretation we did not have accurate data on the productivity of wells (within the studied «cube» all the wells are more or less productive). The available information testifies to the fact that the highest productivity is observed in the wells $13,11,9,20,2,5,4,7,8$, 10. The wells of this group according to qualitative DFM interpretation are located either in the outline of the channel or near the boundaries of the blocks (the boundary of an active block should possess the highest permeability). After productivity of all wells is defined more accurately, the maps of initial pressures and current pressures (to date) are constructed, we will have an opportunity to make more precise analysis of the rules of productivity distribution in 
these or those stratigraphic sequences and to analyze relationship between the found rules and the elements of dynamic fluid model. It is quite probable that the current distribution of the anomalous pressure is different from that before beginning of the field development. Therefore, the calculated estimate of the current anomalous pressure reflects new conditions in the production zones penetrated by the wells which were abandoned in the past (the wells with lost productivity). In this connexion, the node of the lines $\mathrm{A}$ and $\mathrm{C}$ seems the most prospective and suitable for testing by drilling by analogy with the node of the lines A and B where all three wells $(3,4$, and 5) have the highest productivity. The final recommendations can be formulated after the stage of quantitative interpretation.

\section{The initial conditions for the stage of quantitative interpretation.}

The central condition for solving the problem of estimating the fluid pressure and permeability on the derived estimates of the anomalous pressures is the construction of a productivity «cube» in several variants:

a) a cube of initial fluid pressures at the time of well testing,

b) a cube of accumulated productivity,

c) a cube of average productivity reduced to one time interval.

With account for this information the stage of quantitative interpretation will include solution of the direct problem with the use of Darcy's equation aiming at prediction of permeability on the specified pressure values with the variants of initial pressures (at the moment of well drilling) and above-hydrostatic current pressures (on the seismic data with account for calibration by well tests).

\section{PROCESSING AND INTERPRETATION OF SEISMIC DATA IN BOONSVILLE FIELD.}

The Boonsville project area is located within the limits of Fort Wortch Basin, Texas and related to the type of midcontinent reservoirs with gas pools (the main product) and oil pools in the Bend Conglomerate interval. The oil-and-gas-bearing capacity of the region was determined in 1956, and development of gas and oil was started that time. The oil and gas pools are of stratigraphic type and located within the shelf zone with a system of channels and bars filled mainly with sandstones and mudstones of different types. The main oil inflows were obtained from Caddo sequence, while the main gas inflows were obtained from Vineyard sequence (the main target zone in the past). 3D seismic surveying in this field had to solve problems related to mapping of karst zones (that is, dissolution of buried Ellenburger carbonates)and sandy reservoirs of the Caddo sequence (by using the seismic attributes such as instantaneous amplitude and frequency). Also a joint analysis of the parameters of well productivity and the results of detailed stratigraphic analysis on the 3D seismic images should be fulfilled.

DFM processing and interpretation of the initial stacked «cube» was carried out according to the same principle which was used previously in the Stratton field, but in a more expanded variant to account for the necessity to detect local regions of karst process development. Besides, rather detailed information on the parameters of well productivity is available in given case, so we have an opportunity to fulfill analysis of the relationship between well productivity parameters and the anomalous pressures estimates derived from the seismic data. 
Processing of the initial «cube» using the DFM technology.

In this case the general scheme of processing coincides with the scheme applied in the Stratton field :

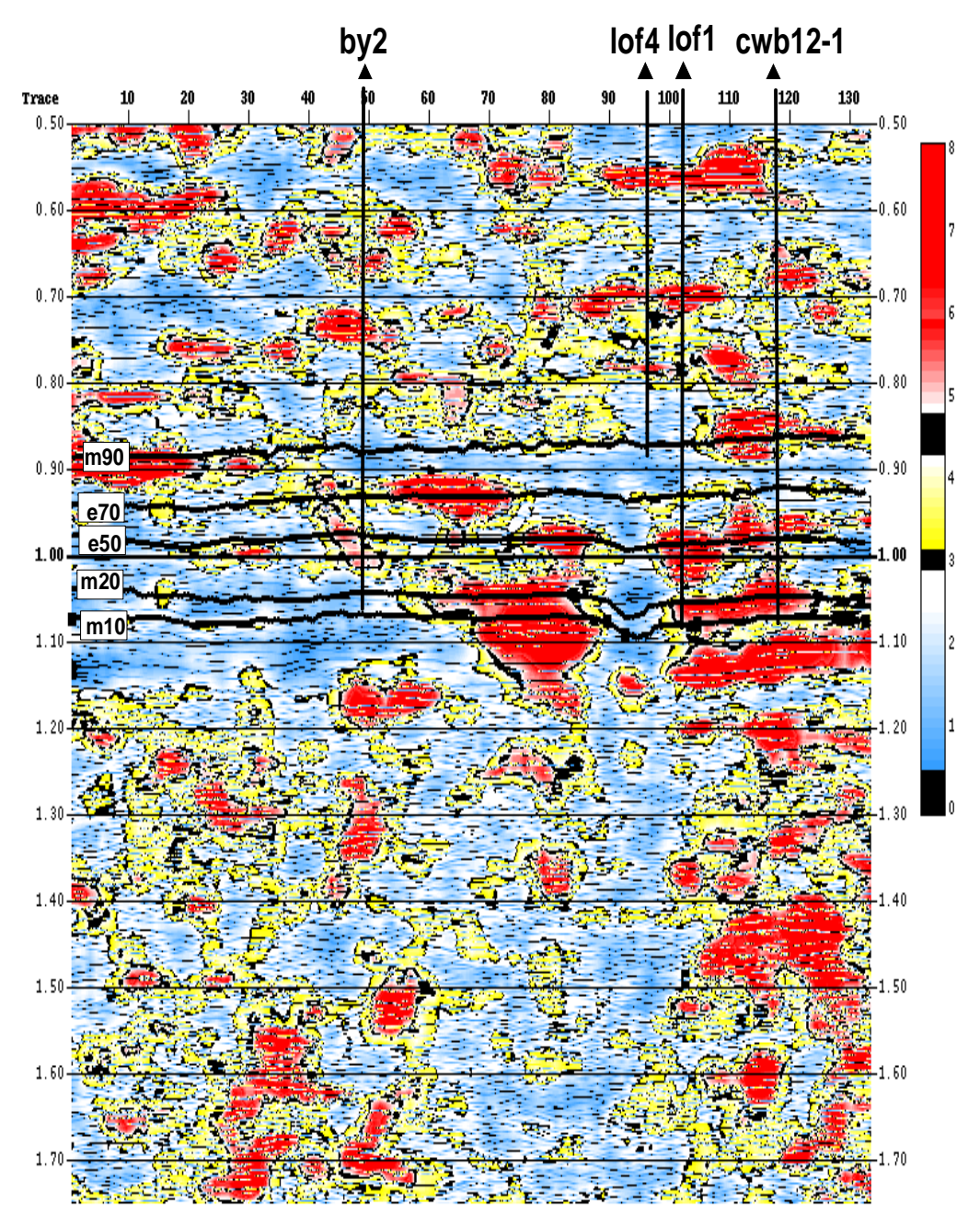

Fig.1. Cross section, L2, picked horizons (defined by black lines).

Pressure anomalies (color, red - low pressure, blue - normal lithostatic pressure)
- the main horizons within the producing interval have been picked (Fig.1, horizons $\mathrm{m} 90$, e 70 , e $50, \mathrm{~m} 20$, $\mathrm{m} 10$ ),

- estimates of the anomalous pressures have been derived for the picked horizons,

- estimates of the anomalous pressures have been derived for a series of time intervals,

- estimates of the anomalous pressures have been determined in vertical sections for several selected directions,

- estimates of the anomalous pressures have been calculated through the whole «cube» for horizontal slices with the interval of $\Delta \mathrm{t}=4 \mathrm{~ms}$.

Taking into account the specific character of the problems to be solved in this field, the mentioned scheme was supplemented with the DYNAMIXS procedure (in correspondence with the ratio 5) and the procedure for calculation of the integrated pressure estimate (the ratio 6). Thus, all the processing results are represented by the following maps and sections:

1. The maps of anomalous pressure estimates for the selected horizons in the DYNAMIXt and DYNAMIXs variants (Figs. 2 - 6) with the identifiers PT and PS respectively (for example, PTm90 and PSm90 denote the results of calculations using DYNAMIXt and DYNAMIXs schemes correspondingly).

2. The maps of summed estimates of the anomalous pressures for certain horizons (PS $=$ $(\mathrm{PSm} 90+\mathrm{Pse} 70+\mathrm{Pse} 50+\mathrm{PSm} 20+\mathrm{PSm} 10) / 5)$ and the map of horizontal maximum gradients of this estimate (grPS). The maps are shown in Fig. 7. 
3. The maps of integrated estimates of the anomalous pressures (in correspondence with the ratio 6). Fig. 8 shows all intermediate maps, while fig. 9 shows the final map of the integrated estimate of the pressure for seismic horizons $\mathrm{m} 90$ - $\mathrm{m} 10$.

4. The maps of interval estimates of the anomalous pressures derived with the aid of DYNAMIXs scheme in the time intervals 820-1200 ms (fig.10), 1200-1500 ms (fig.11), 1500-1800 ms (fig.12) .

5. The vertical sections of the anomalous pressure estimates derived with the aid of DYNAMIXs scheme for selected directions (Figs.13-16).

Interpretation of the results (the stage of qualitative interpretation).

The general analysis of the results of anomalous pressure estimation with the aid of two calculation schemes in given case brings us to a definite conclusion that the DYNAMIXs scheme is suitable as it gives higher degree of detail of the anomalous pressure estimates for separate horizons as well as in vertical sections. The anomalous pressure estimates derived with the aid of this scheme were selected for final interpretation. Figs.2-6 can be used for comparative analysis of two mentioned processing schemes, and one can conclude that a number of details in the anomaly outlines and their spatial orientation better correspond to the stratigraphic situation, practically for all horizons. Such detail can be explained by higher quality of initial seismic data (first of all, preservation of a wide frequency range), than for example in the previously considered Stratton field. The resulting maps constructed with the use of DYNAMIXS scheme are displayed in an enlarged form in the figures with the index "d". The map for Caddo horizon (upper) in fig. 4 (PSe50) attracts our attention. A decompression anomaly of typical elongated shape (the red color) most likely confirms the presence of a sandy body in the form of a bar or channel, which is one of the main gas and oil reservoirs in given case. The reservoir is located above given horizon and throws a sufficiently bright «shadow» to it. There are strong compression anomalies in the maps for underlying horizons (Figs.5 - 6), and these anomalies are most likely related to the effects of karst process development in more deep intervals of the carbonate sequence.

The generalized analysis of the maps of summed estimates of the anomalous pressures on the seismic horizons, the maps of the interval estimates, the maps of integrated estimates, and the vertical sections (Figs. 7-16) allow for quite definite scheme of dynamics of given region to be built. The primary map for building this scheme is the map of the anomalous pressure estimates in the interval 1,500-1,800 ms (fig.12). Here, in the center, we see a clear anomaly of compression, and this anomaly in given stratigraphic situation can be interpreted as the zone of a regional fault. This fault's activity determined basin's development in essence, first, generation of a sandy buildup in Caddo, and second, contributed to formation of karst in the carbonate sequence, resulting in turn in anomalous transformations in the strength structure and in destruction of the rock mass also in the overlying sequence (Vineyard, Runaway - horizons $\mathrm{m} 10, \mathrm{~m} 20$ and others). The eastern edge of this anomaly is reliably tracked in vertical sections of the anomalous pressure estimates, and along the line " $\grave{\mathrm{A}}$ " we see a vertical boundary in the form of a narrow, clearly drawn zone. In given case the outline of this zone is identified in all vertical in-line sections, and the position of the boundary " $\mathrm{A}$ " as the active contact between two large dynamic blocks is apparent. Analysis of the maps and vertical sections in this context also allows for detection of the boundaries of the blocks of lower levels: A, C, and D. 
a Map of instantaneous amplitudes along the horizon m90 [ Am90 ]

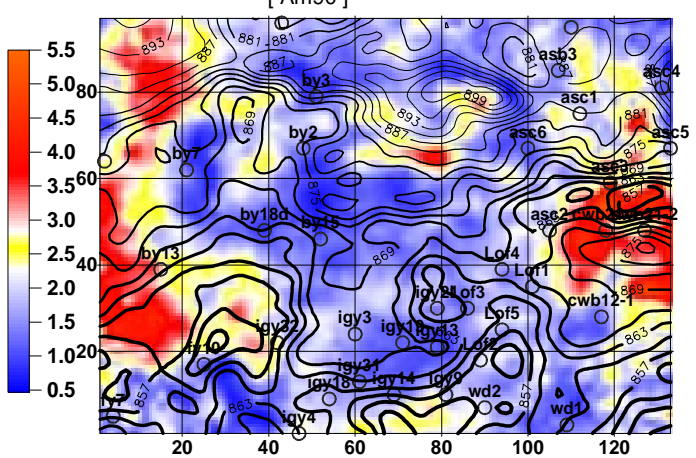

C Map of pressure anomalies along the horizon $\mathrm{m} 90$

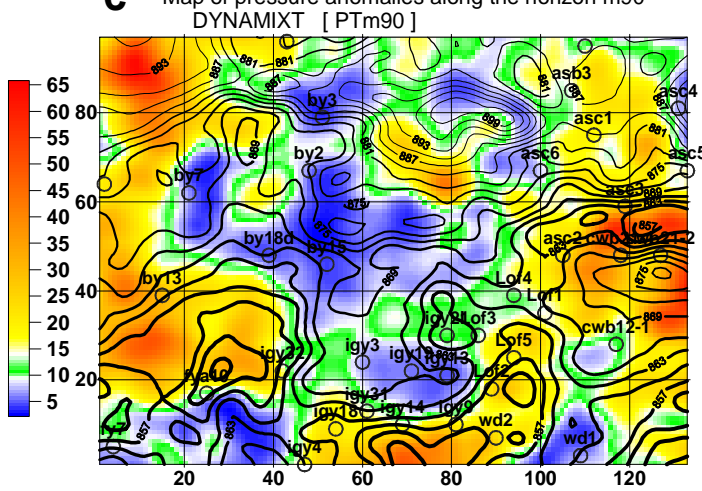

b Map of instateneous frequencies along the horizon m90

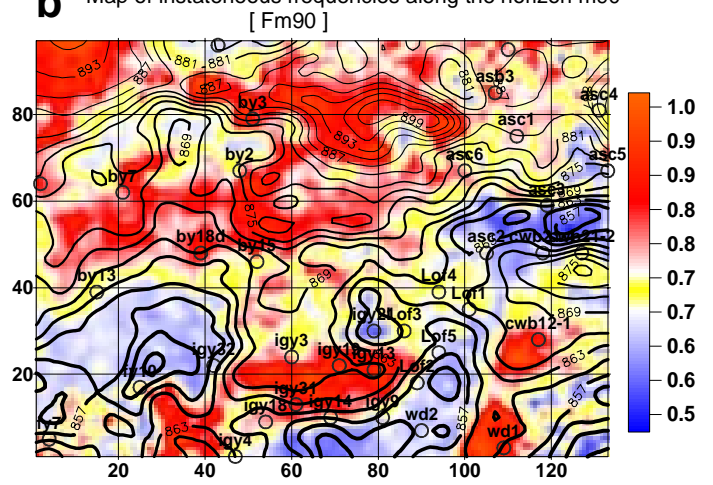

d Map of pressure anomalies along the horizon $\mathrm{m} 90$

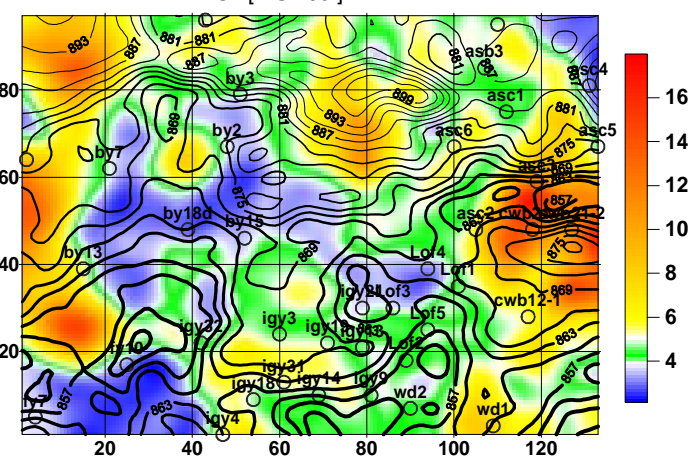

Fig.2. Maps of inital attributes and pressure anomalies. Horizon m90.

a Map of instantaneous amplitudes along the horizon e70 [ $\mathrm{Ae} 70$ ]

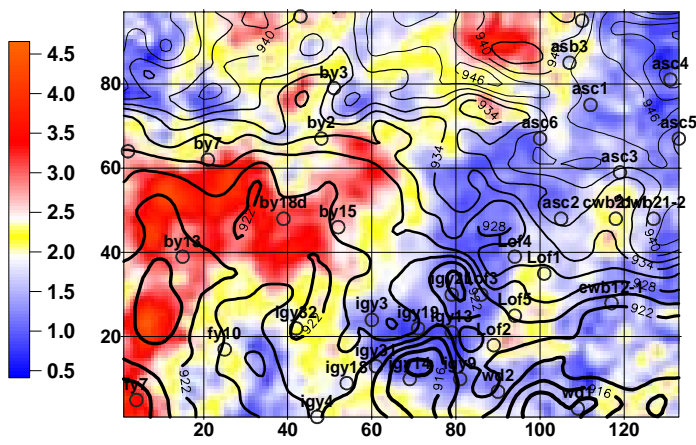

C Map of pressure anomalies along the horizon e70

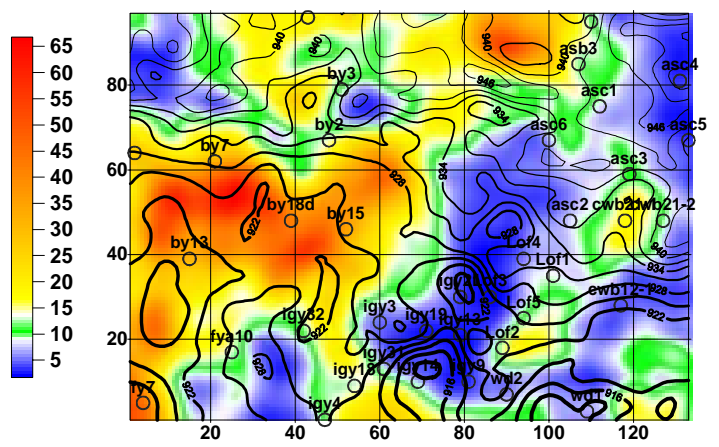

b Map of instateneous frequencies along the horizon e70 [ $\mathrm{Fe} 70$ ]

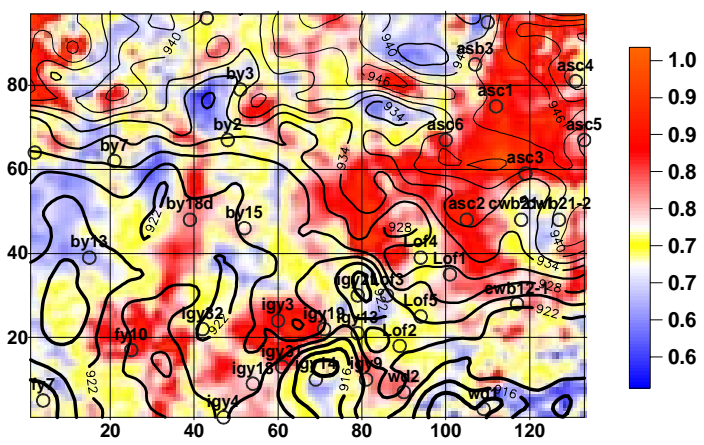

d Map of pressure anomalies along the horizon e70

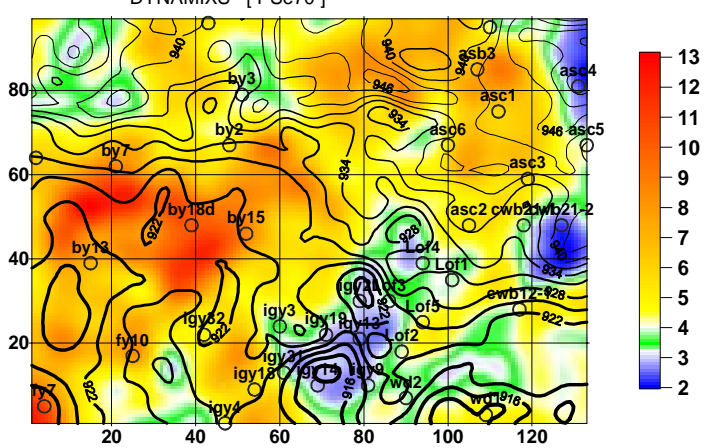

Fig.3. Maps of inital attributes and pressure anomalies. Horizon e70.

Final Technical Report October, 2002
Michigan Technological University

DE-AC26-98BC15135

Page 156 

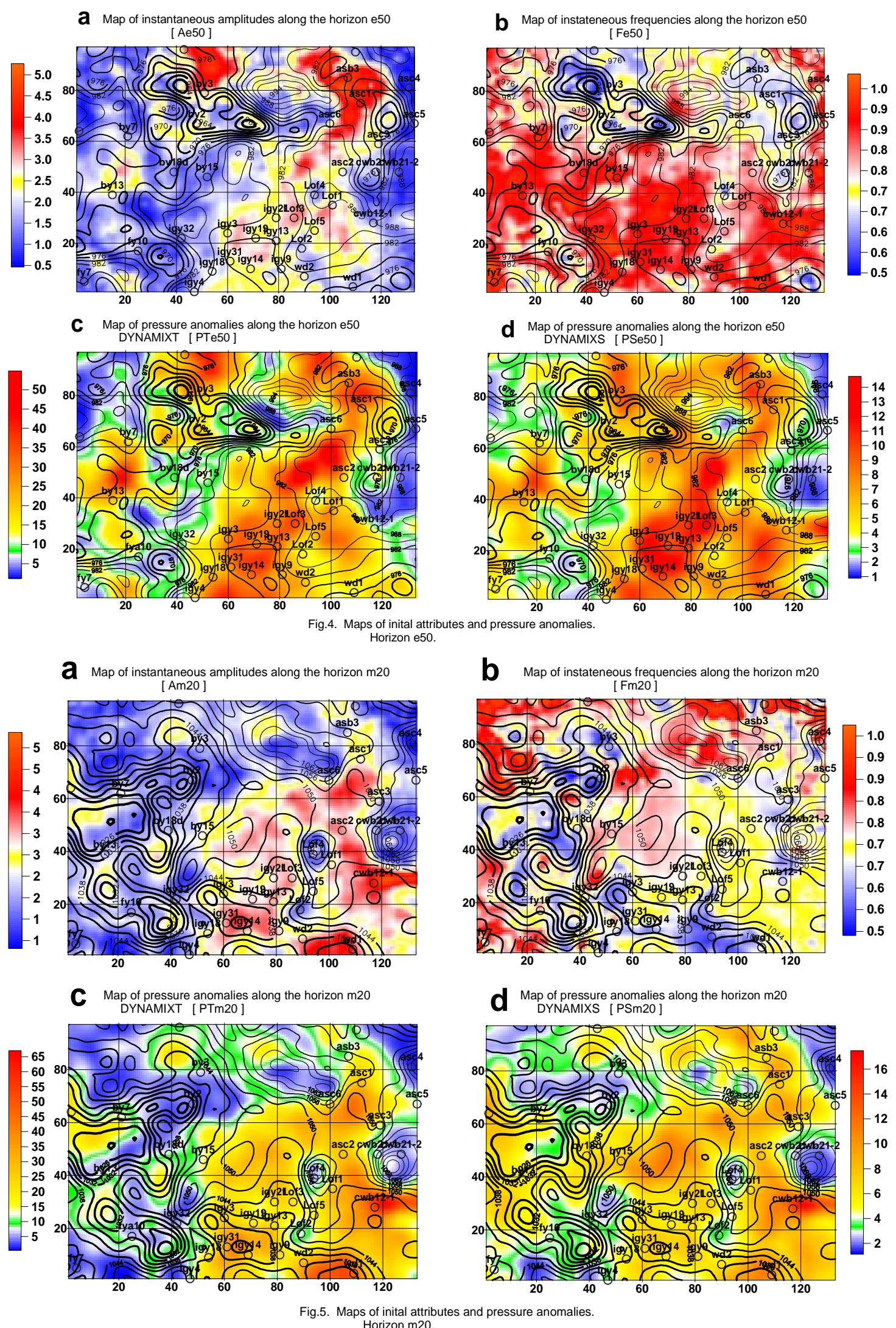
Horizon m20. 


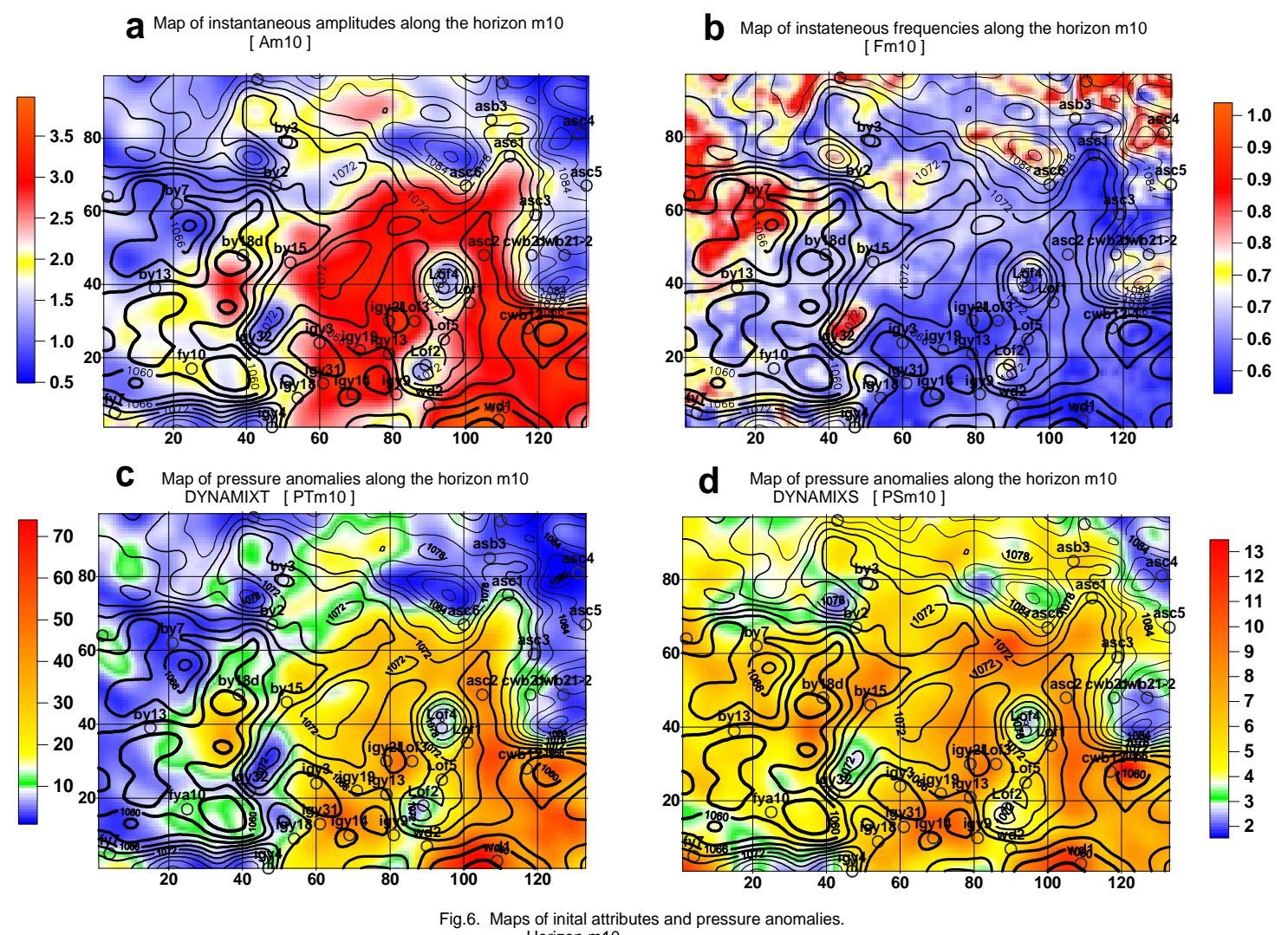

Fig.6. Maps of inital attributes and pressure anomalies. Horizon m10.

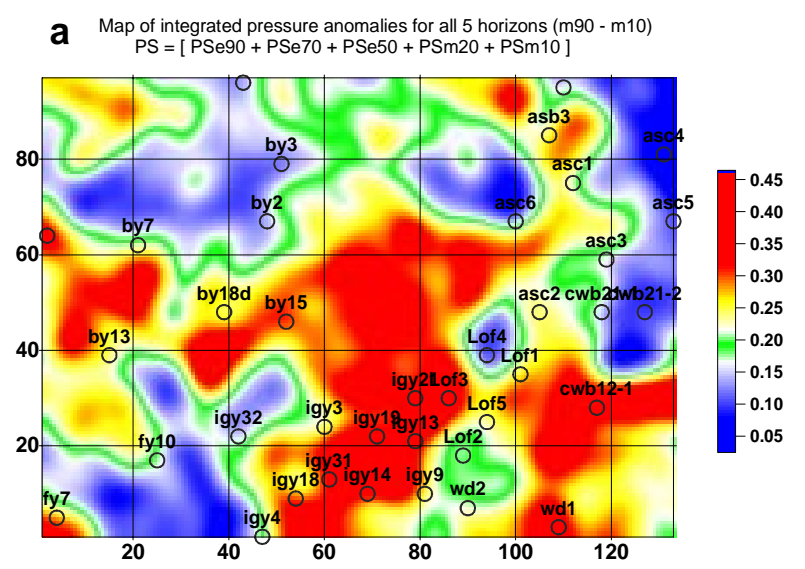

b Map of maximum gradients of pressure anomalies for all 5 horizons
grPS $=[$ grPSe90 + grPSe $70+$ grPSe $50+$ grPSm20 + grPSm10 $]$

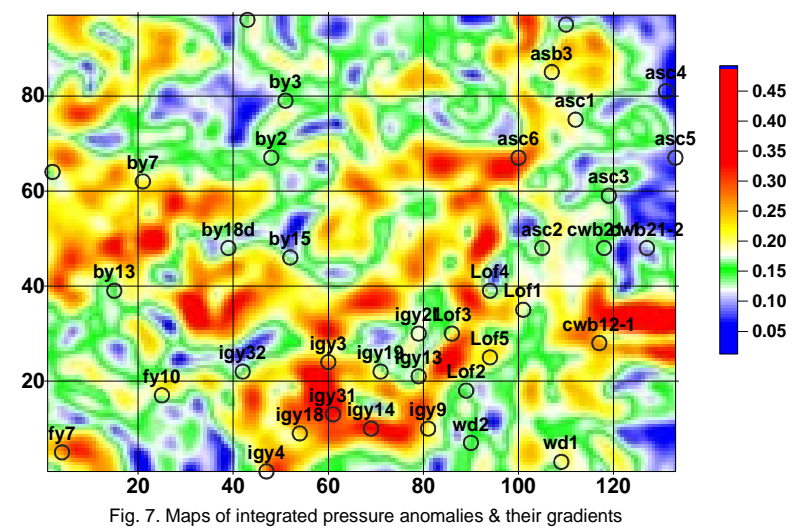

Final Technical Report Michigan Technological University

DE-AC26-98BC15135 

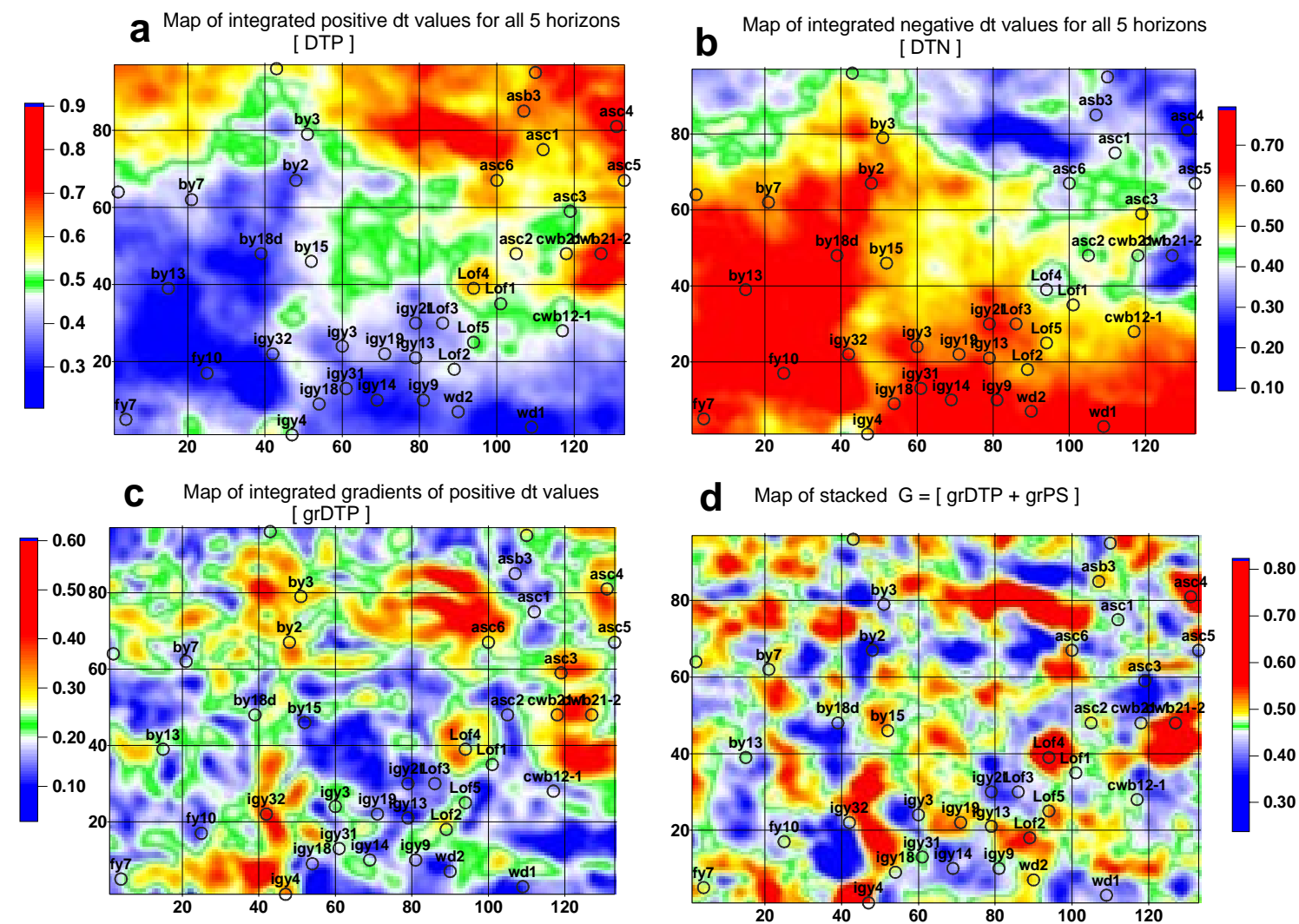

d Map of stacked $\mathrm{G}=[\mathrm{grDTP}+\mathrm{grPS}]$

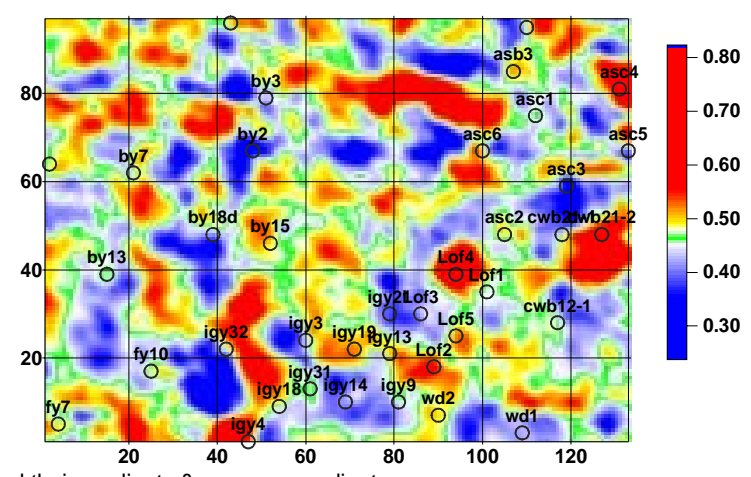
( horizons e90-m10)
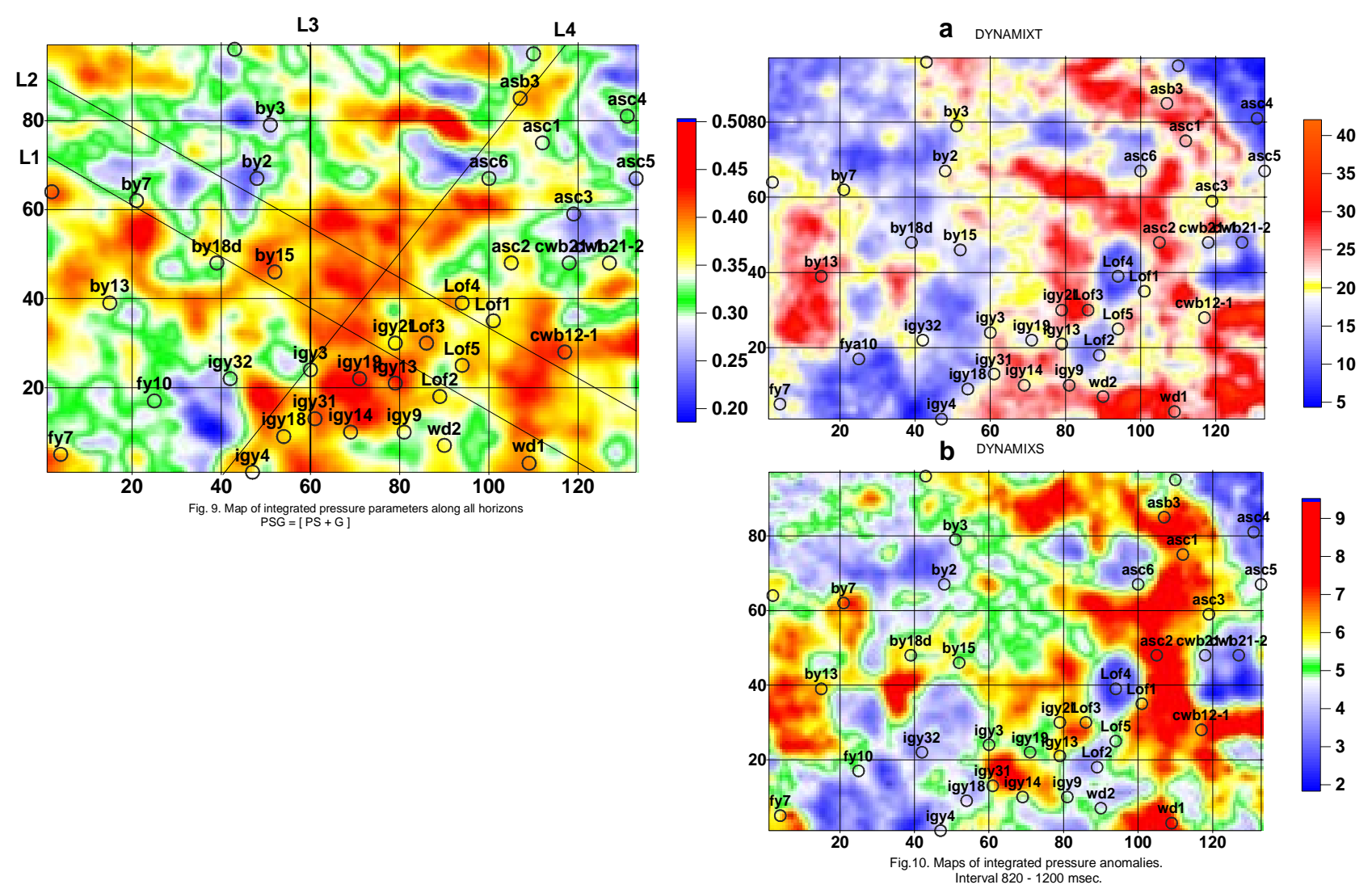

Final Technical Report Michigan Technological University

DE-AC26-98BC15135

October, 2002

Page 159 


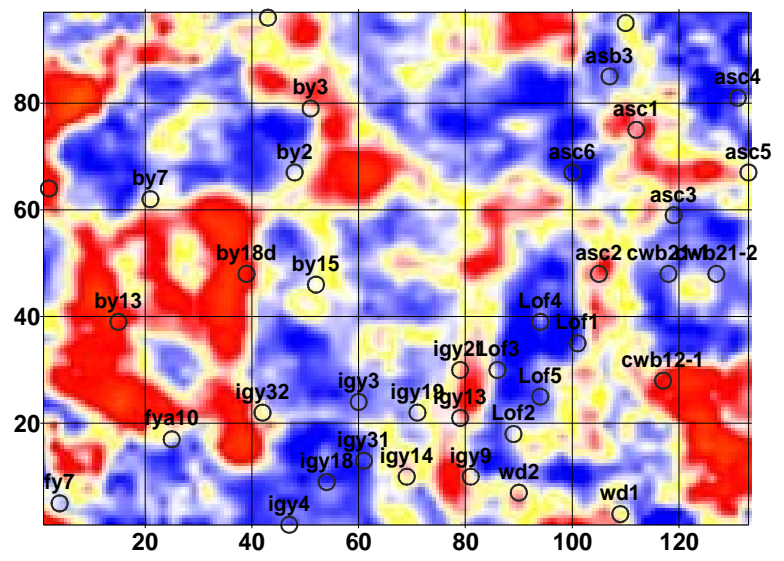

b DYNAMIXS

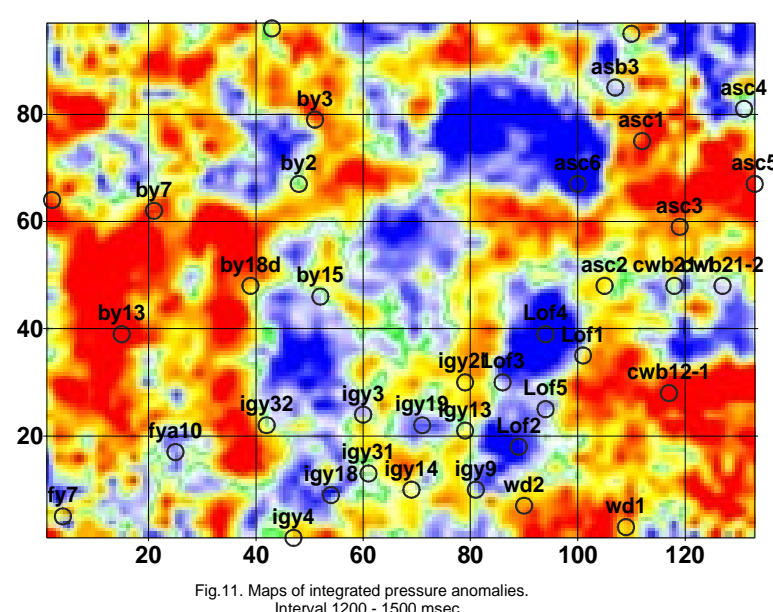

Interval 1200 - $1500 \mathrm{msec}$.

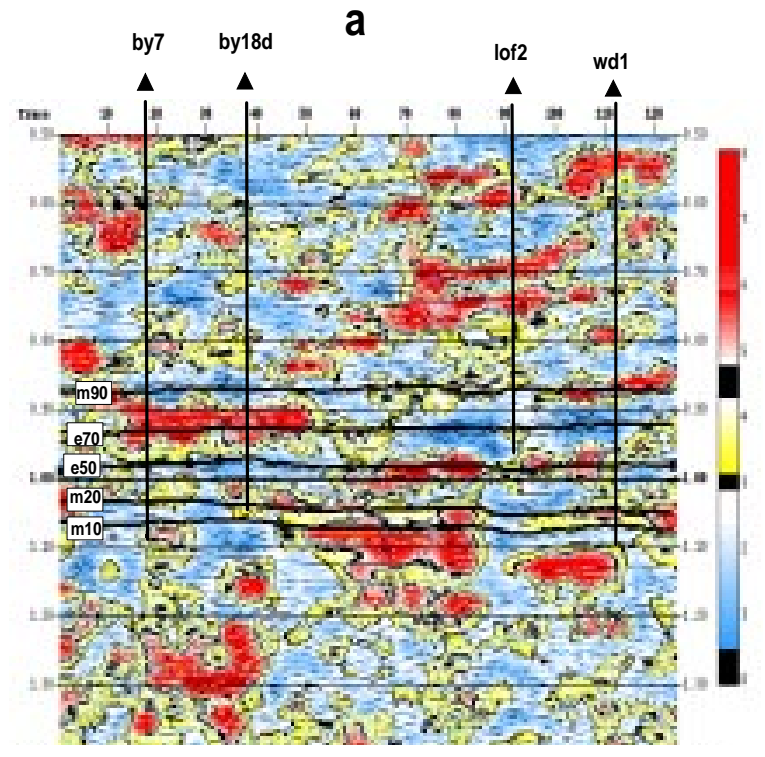

a DYNAMIXT
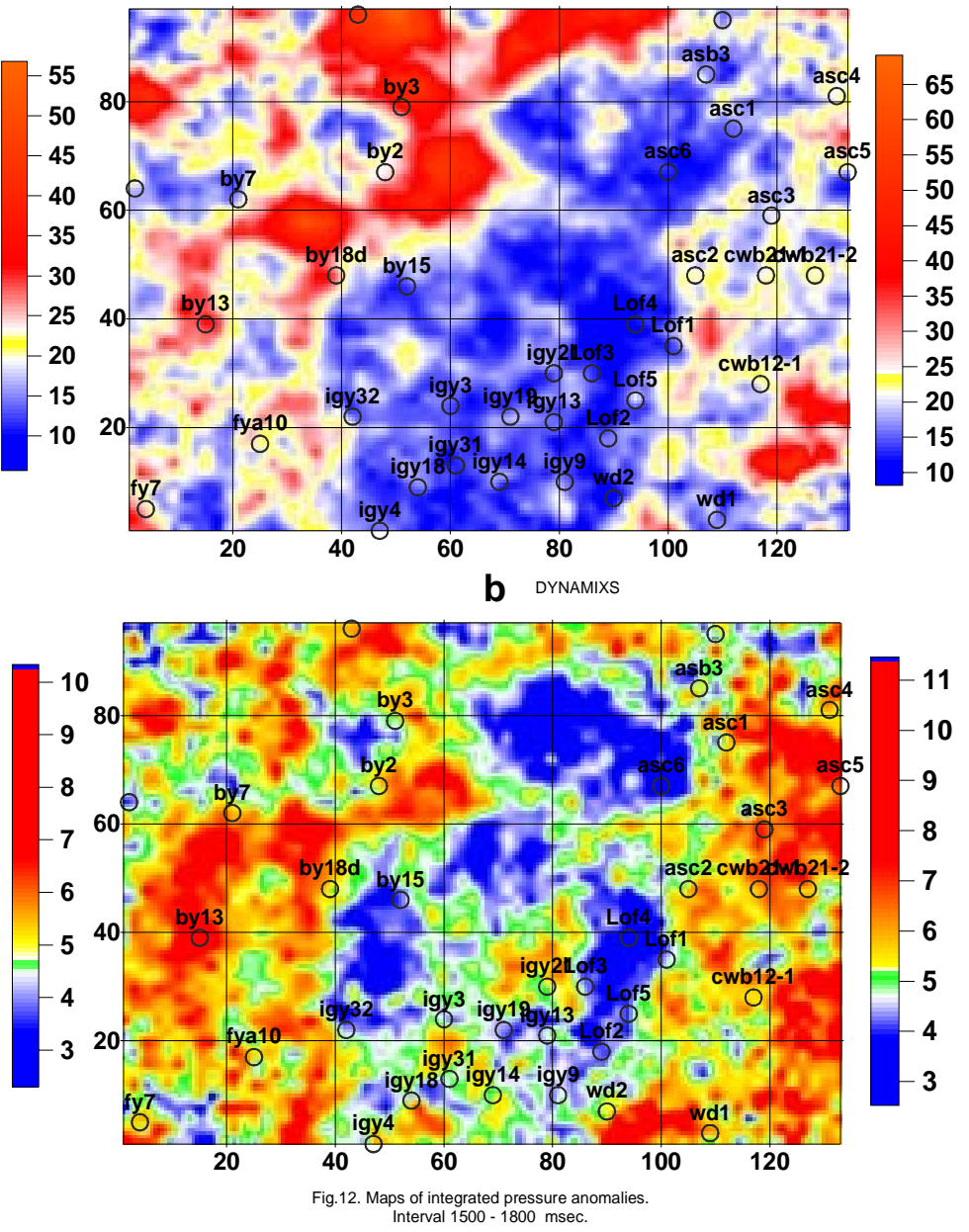

b

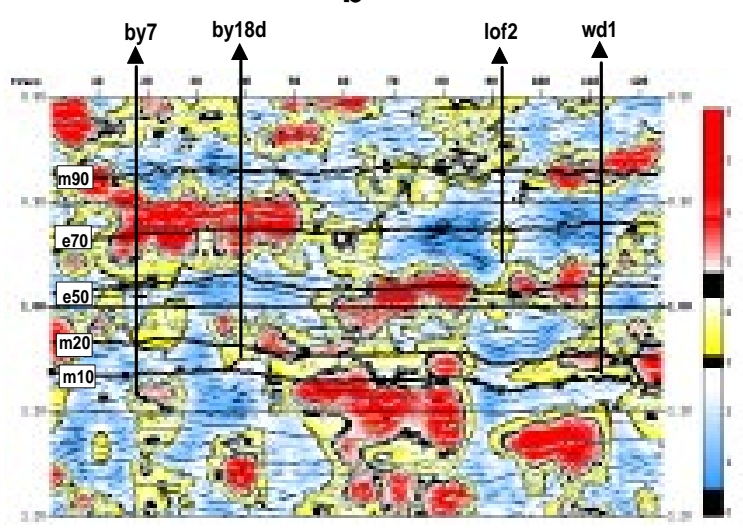

Fig. 13. Cross section along L1.

Pressure anomalies DYNAMIXS 
a
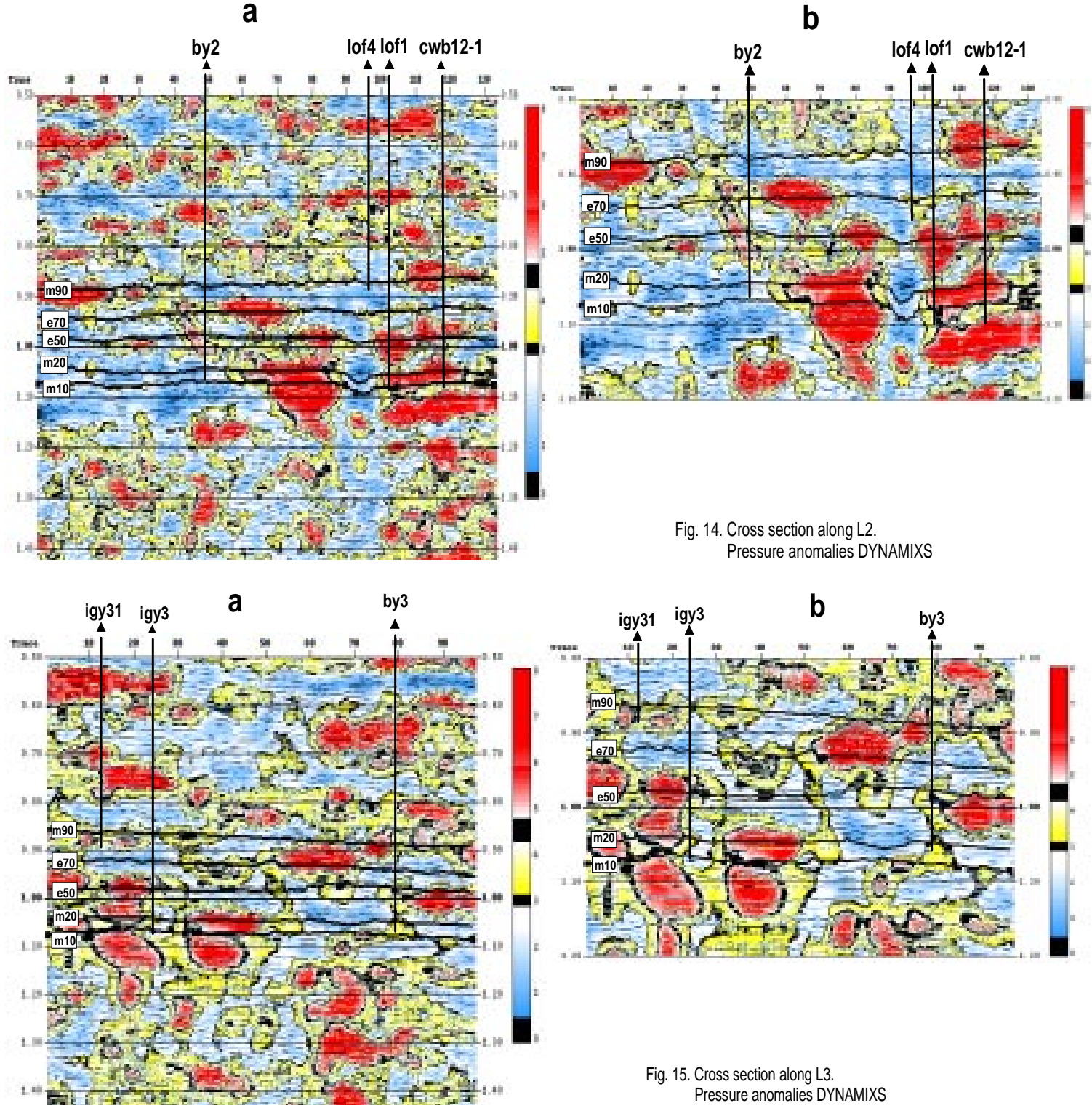

Fig. 14. Cross section along $L 2$.

Pressure anomalies DYNAMIXS

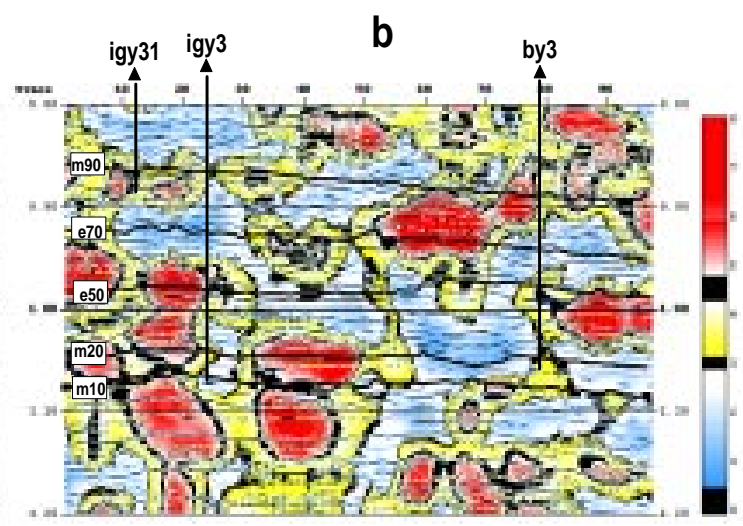

Fig. 15. Cross section along $L 3$.

Pressure anomalies DYNAMIXS
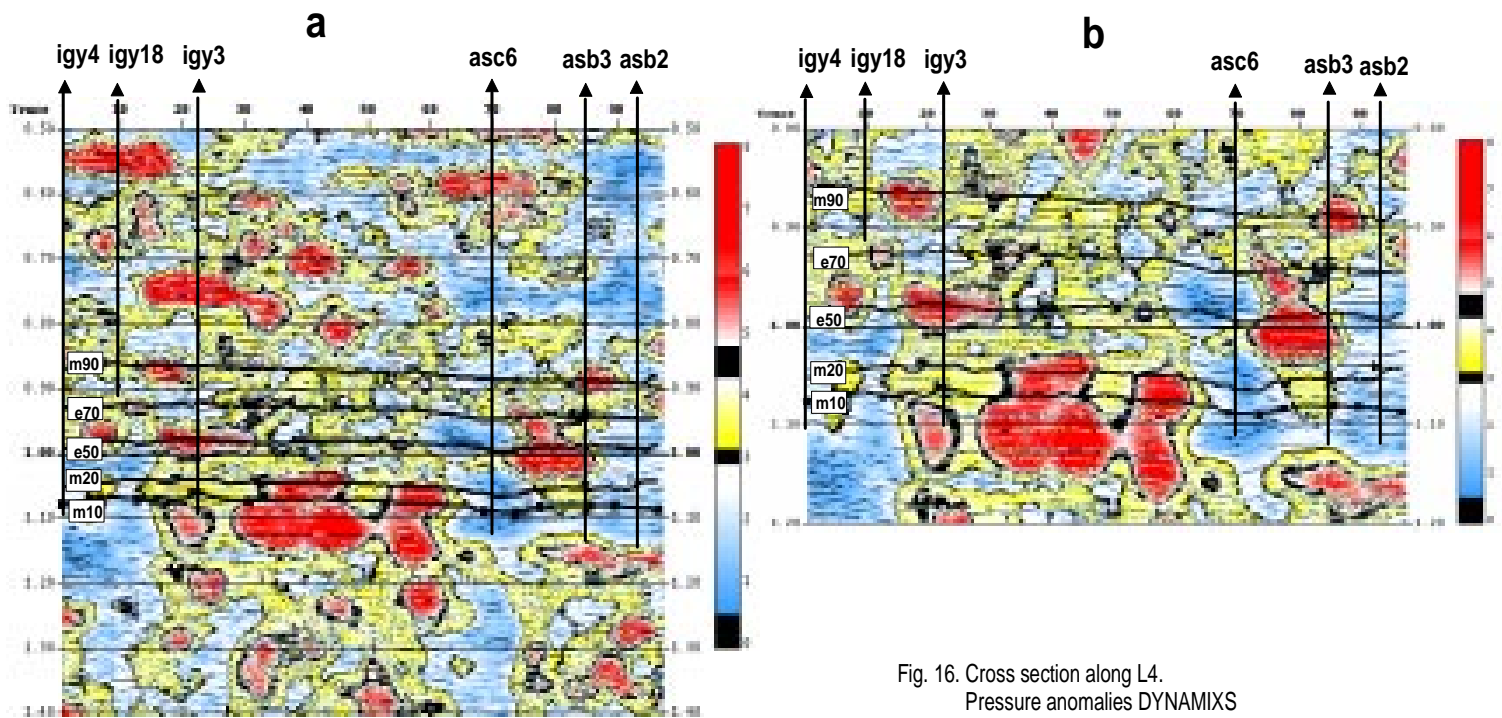

Fig. 16. Cross section along $L 4$

Pressure anomalies DYNAMIXS 
All the mentioned lines form a regular scheme of the block dynamics of the basin, and this scheme does not contradict to typical pressure anomalies in the maps constructed for seismic horizons or time intervals. Especially, contrast anomalies of compression and decompression are observed along the boundary " $\grave{A}$ ". This may be an indication of altered high-permeability zones generated by karst processes. Besides, location of the line "À" completely explains possible

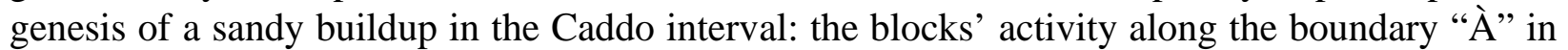
the period of sedimentation of this stratigraphic interval should have lead to the natural change of the surface morphology, thus, causing quick accumulation of sandy sediments along this boundary.

The determined scheme of block dynamics and the maps of anomalous pressures calculated only on the seismic attributes help in stratigraphic interpretation on the one hand. It also allows to estimate fluid saturation on the ground of dynamics by comparing the elements of the scheme of block dynamics with the detailed data on the productivity of representative number of wells with known history and fluid parameters on the other hand. For that purpose, it was necessary to superimpose a number of parameters, which give information about the real fluid saturation in these intervals, on the maps of integrated estimates of the anomalous pressures for all horizons or for producing intervals. The available data allows the following parameters listed in the tables of well productivity to be selected for analysis:

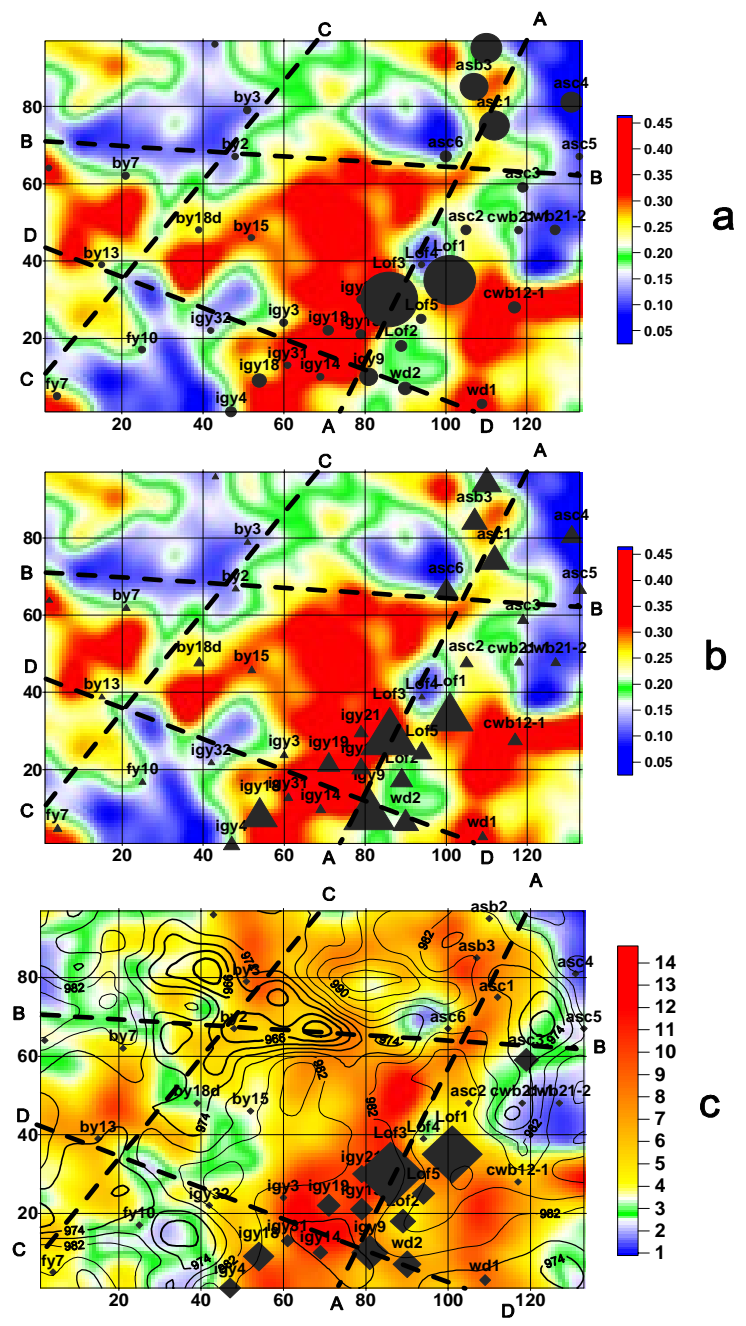

- cumulative volume of the product recovered during history of the well (cum. gas and oil),

- average annual productivity of the well (gas/j , oil/j),

- initial formation pressure at the moment of well testing ( $\mathrm{p}$ init),

- cumulative volume of water,

- total time of well exploitation,

- generalized porosity of the rocks in the interval,

- cumulative volume of the product for separate stratigraphic intervals.

Fig.17 shows the maps of summed estimates of the anomalous pressures on the seismic horizons ("a" and "b") and the map of anomalous pressure estimates on the horizon e50 - Caddo ("c"). The values of cumulative volume of the oil recovered during the whole history of exploitation of all the wells are superimposed on the fragment "à", while the average annual oil recovery is superimposed on the fragment " $b$ ". The volume of recovery from the Caddo interval is shown in the fragment "c". The general conclusion is the

Final Technical Report

Michigan Technological University

DE-AC26-98BC15135

October, 2002

Page 162 
following in this case: oil-bearing capacity of the whole producing interval, including Caddo interval, is associated with the most active contact zone along the boundary " $\mathrm{A}$ ". This can be explained by two reasons:

- a vertical region of the medium independently from its stratigraphy and lithology in the vicinity of the contact of active blocks in the plane "A" possesses a high permeability and serves as the main migration channel from deep intervals of the basin (ascending migration) or from the periphery (lateral or horizontal migration),

- the reservoir intervals are located in the zones of maximum decompression (the red color) in direct closeness from the active contact. This determines the maximum localized voidness of the medium in corresponding lithological conditions of the rock mass and the maximum fluid pressure in it (the maximum value of decompression "automatically" determines the maximum fluid pressure in the observance of other favorable conditions).

A similar situation can be observed for gas recovery (fig.18), but here (which was to be expected) the productivity if the interval extends essentially farther from the contact "À" along the lower-rank boundaries of this contact, namely "Â" and "D". Fig.19 shows the parameters of initial pressure (fragment "à"), cumulative volume of water (fragment " $b$ "), and the generalized

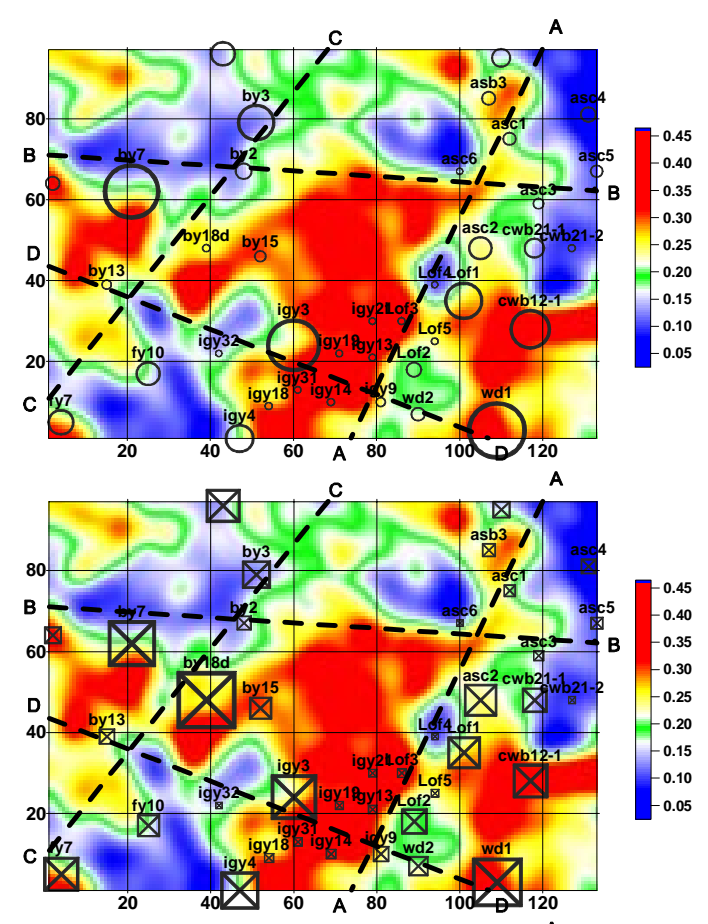

porosity of rocks (fragment " $\tilde{n} ")$. These parameters are superimposed on the integrated estimate of the anomalous pressure (fig. 9) for the whole producing interval. All the parameters in their entirety confirm that the area in the a vicinity of the active contact " $A$ " has higher permeability and productivity, which is an indirect confirmation of the results represented in Fig. 20, fragment "à": the largest time period of well exploitation is observed in the vicinity of the most active node near the crossing of the boundaries " $\mathrm{A}$ " and "D". The highest recovery of gas for the "Vineyard" interval is shown in the fragment " $b$ " of the same figure, and this b point is precisely associated with the position of the system of contact boundaries.

The fulfilled qualitative analysis of the relationship between the well productivity and the parameters of the dynamic condition of the basin has the very preliminary character and requires a quantitative confirmation on the basis of modeling of the Darcy's flow in the defined scheme of block dynamics. We see a connection between the parameters of reservoir productivity and the parameters of the real dynamic condition of the basin for the purposes of stratigraphic interpretation as well as for evaluating oil-andgas-bearing capacity of a basin.

Final Technical Report

Michigan Technological University

DE-AC26-98BC15135

October, 2002

Page 163 

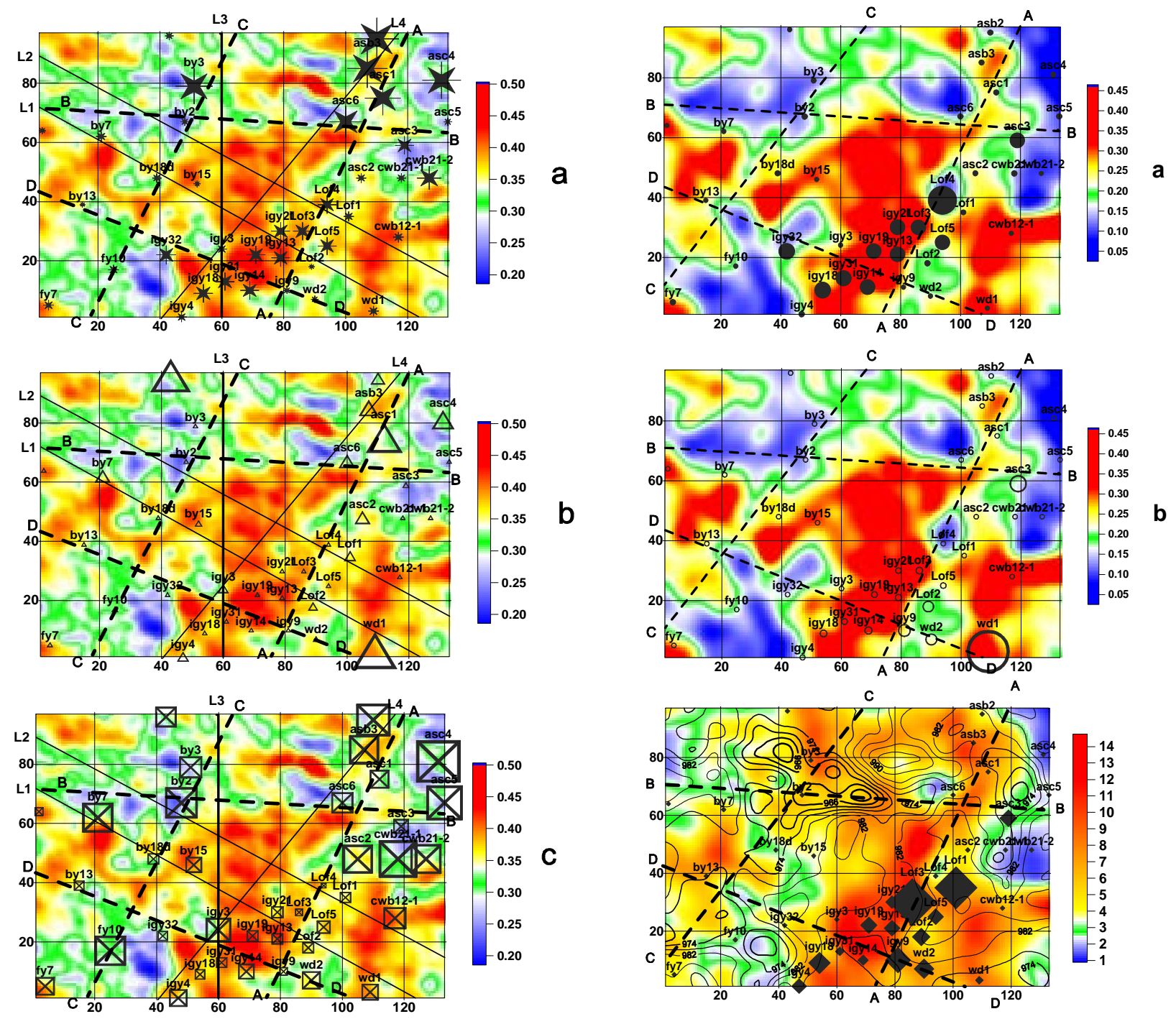

Thus, the derived estimates of the anomalous pressures and the scheme of block dynamics can serve as the physical basis for evaluating oil-and-gas-bearing capacity of the area under consideration. Therefore, the recommendations in this case are the following: the highest fluid pressure should be observed within the vicinities of the contacts between active blocks and should be associated with local regions of reduced total ground pressure (decompression regions).

\section{CONCLUSIONS.}

The results of the first stage of processing and interpretation of seismic data with the aid of the DFM technologies in the Stratton and Boonsville areas can be formulated as several general and interconnected conclusions:

General dynamics of a sedimentary basin determine the stratigraphic bodies, the principle of their detection on seismic data should be based on estimation of the current dynamic condition of the basin and discovery of local regions of anomalous pressure in the studied intervals. 
Estimation of the anomalous pressures on the seismic data allowed for objective detection of local bodies, such as «channel» or «bar», in the areas under consideration.

Distribution of the anomalous pressures within the studied intervals of the basin was determined exclusively on the seismic data. This distribution is in agreement with the actual parameters of well productivity, thus, indirectly confirming DFM results.

\section{RECOMMENDATIONS FOR FURTHER ANALYSIS}

We advise an additional quantitative stage of interpretation which can give more detailed information about productivity of the reservoirs in different intervals in the studied areas. With account for possible remigration processes caused by exploitation of the fields, information on the residual contour of oil or gas saturation can be extracted after the stage of quantitative estimation of the parameters of the fluid flows. This will allow for the formulation of a number of practical recommendations (drilling of new wells).

To fulfill the final quantitative interpretation in 1999 - 2000 it is necessary to develop spatial models of productivity of the studied reservoirs in the form of the fluid parameters «cubes» built on the available borehole data and coincident with the format of the seismic «cube». This will allow for objective calibration of the "seismic cubes" of the anomalous pressure estimates, and these "productivity cubes" together with the defined geometry of block dynamics will allow for quantitative solution to the Darcy's problem for different initial conditions. Solution to this problem will help formulate a series of practical recommendations for further development of the Stratton and Boonsville fields. 


\section{Dynamic Fluid Method (DFM) interpretation of the oil and gas potential of the Wamsutter field.}

\section{INTRODUCTION}

Here, we have applied our technology and software DYNAMIX ${ }^{\mathrm{TM}}$ based on the Dynamic Fluid Method (United States Patents № 5,796,678; № 6,028,820; other patents and licenses pending) to recognize as best as we could subtle and general features of the Wamsutter field.

The sedimentary sequences formed in the shallow-sea conditions are characterized, as a rule, with a thin-layer structure accompanied by complex spatial configurations of different stratigraphic units, each - with a limited size (with respect to seismic wavelength) both in thickness and spatial extent. In such conditions, the geometrical (structural) and the fluidodynamic parameters of oil and gas pools are determined by a number of factors apparent in the Wamsutter region. Almond formation is defined by a complex mode of deposition in the shallow-sea conditions.

As the existing methods and technologies of seismic data interpretation are based mainly on the geometry and the properties of reflecting boundaries, the solution of the problem of stratigraphic zonation within the target intervals of the subsurface is automatically limited by the resolution of seismic signals in the presence of interference. In such sedimentation setting, traditional techniques of seismic data interpretation allow for reliable definition of rather thick formations, but these techniques are practically useless for a detailed subdivision of the subsurface into units with thickness less than half of the seismic wavelength.

For more detailed analysis of the reservoir properties of each formation containing subtle stratigraphic features, traveltime parameters of seismic signals should be used in combination with other attributes such as instantaneous amplitudes and frequencies. We believe, that the attributes of seismic signals are related to the dynamic state of the medium on both sides of the reflecting boundary. In other words, these seismic attributes depend on the value of the total ground pressure acting at the reflection point. If we are able to estimate deviation of the pressure from normal lithostatic value (i.e. the anomalous pressure value), for example, along the roof of a thick formation or in certain seismic sequence, then we can perform the following predictions:

Anomalous pressures in the reflection points can be caused by modern dynamic processes occurring in sufficiently large regions of the basin (block structure of the subsurface), as well as by lithological heterogeneity in the closest vicinity of this reflection point. And "sensitivity" of seismic waves to the pressure at the reflection points ensures detection of a feature which is much smaller in size than the wavelength. For instance, a sand lens with the thickness of about several meters and the width of several tens of meters causes a significant change in the distribution of the total pressure in the surrounding medium at the distances up to one hundred meters from the lens.

Independently of the reasons causing an anomalous deviation of the total ground pressure, a region of the subsurface with anomalously low pressure will be favorable for the accumulation of a fluid.

A relative estimate of the anomalous values of the total ground pressure can be obtained using the DFM technology of seismic data interpretation. Application of DFM technology allows for 
determination of fluid migration parameters such as relative values of fluid pressure and the structure of modern block dynamics in the "basin-basement" system.

\section{BACKGROUND}

\section{Geologic Setting}

[Adapted from the work and thesis of Anastasia Minaeva, MTU, 2001, summarized in the main body of this report. Figures that are duplicated from the main body of this report are presented here at small scale.]

The study area, the Wamsutter field, is situated in southwestern Wyoming in the Greater Green River Basin (Fig.1). The area of investigation is in the Rocky Mountain foreland structural province, which is comprised of high desert basins surrounded by breached anticlines with Precambrian cores, and thrust-faults.

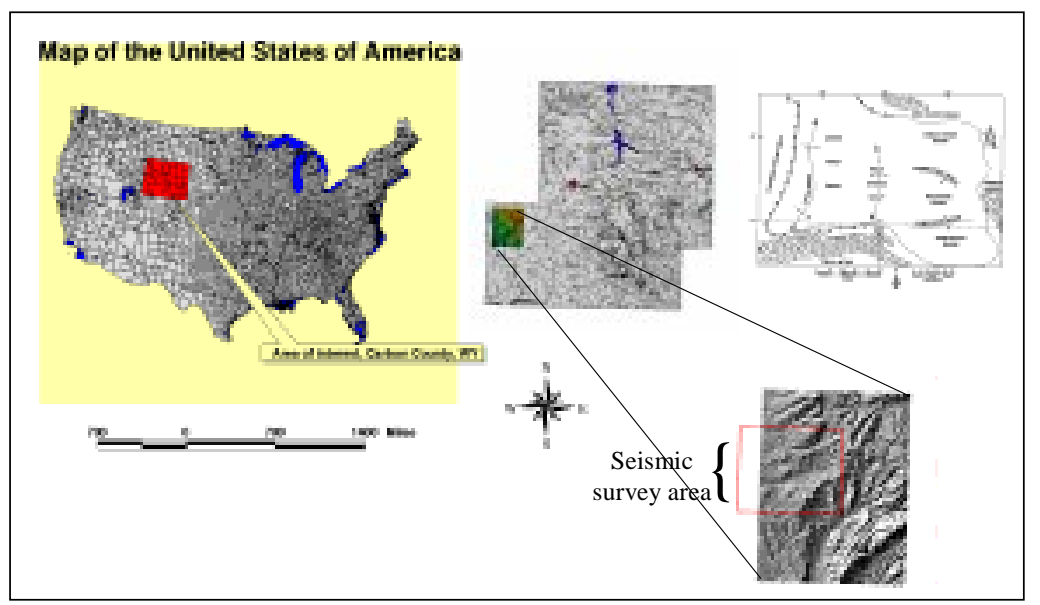

Fig.1. Map of the Wamsutter field.

Greater Green River Basin contains several geologic structures: the Rock Springs uplift, the Great Divide Basin, the Waster Arch, the Washakie Basin, and the Green River Basin. The Wamsutter Arch separates the Great Divide Basin from Washakie Basin. The Wind River thrust, Thrust Belt, Uinta

Mountains, Sierra Madre, and Rawlins uplift define the northwestern, western, southwestern, southeastern, and northwestern margins of the Greater Green River Basin respectively (Jaworovski and Simon, 1995).

It needs to be noted that studied region situated near the Eastern margin of Basin and Range Province. Basin and Range Province and adjacent extension regions in Canada and Mexico cover the area of about 2 millions $\mathrm{km}^{2}$ being the region of multistage extension from Late Precambrian to Present. More and more often Basin and Range Province is considered as the standard for interpretation of extension structures in different parts of the World.

A lot of geological and different geophysical studies revealed geologic setting of the Basin and Range Province and main characteristics of its interior structure. It has, besides unconsolidated deposits, two- and three-storied tectonic structure. The upper layer (upper crust) is composed by consolidated, however, unmetamorphosed sedimentary Upper Precambrian, Paleozoic and Cenozoic rocks and also by abundant magmatic intrusion and volcanic rocks of different ages up to Present. The middle crust (middle layer) is mainly made by same rocks (with increasing the proportion of intrusive ones), but they were metamorphosed under greenstone and locally amphibolite facies and were mylonitized in the upper part. In the most extended regions the middle crust is absent. The lower crust is probably composed by basites with increasing basity downward to Moho that is located at the depths of about $30 \mathrm{~km}$. 
The upper crust of Basin and Range Province is represented by mainly west-south-west and eastnorth-east tilted blocks, quite often complicated by north-north-western dextral strike-slip shear zones. Blocks are classified by size from several kilometers to first hundreds meters depending mainly on the thickness of the upper crust. They are separated by listric, more rarely, linear faults observed by from the seismic only down to a base of the upper crust. The latter is bounded below by subhorizontal fault (detachment fault). It corresponds to the boundary the "brittle" upper crust and plastic middle one, rocks of which were weakened due to large pore pressure of fluids and deformed mainly plastically.

The rocks of the middle crust («core complexes») are cropping out in antiforms forming gneiss domes in windows of the upper plate. Genesis of these structures is interpreted after B.Wernicke (1988 etc) by gently dipping gigantic strike-slip faults extending into the mantle. Later on he and other developed the hypothesis of «rolling hinge». But both hypothesis have kinematic unfoundedness and structure and origin of «core complex» antiforms should be interpreted on the base of a new rheological model of the earth crust (Ivanov, Ivanov,1993), which develops the early proposed one (Smith, 1978; Miller and others, 1983).

It was determined that formation of detachment and movements along it (of the upper plate relative to the middle one) occurred simultaneously and in the same direction with mylonitization of the middle crust at the depth from several meters to several hundreds meters (and even to several kilometers) beneath the detachment. In a number of Basin and Range Province regions, magmatism and metamorphism manifestations associated with extension. Role and significance of thrusts, the weakly studies traces of which are marked only at the eastern boundary of Basin and Range Province, usually had been exaggerated. The indications of a large ductile movement including lateral one of masses of the lower crust and, apparently, of the mantle, synchoronous with extension of the upper and middle crust has established. The contemporary morphology of the Moho discontinuity reveals the ability of the mantle to support the isostatic equilibrium synchronously with the deformation of the crust in spite of rapid extension of the latter.

\section{Trans Seismic International, Inc.}

\section{[The following text is original from TSI]}

The Dynamic Fluid Method approach makes the assumption that the spatial distribution of the anomalous pressures is determined by modern vertical movements of the blocks of the sedimentary cover in the "basin-basement" system.

Consequently, we may assume that larger-scale horizontal movements (in particular, multistage extension in Basin and Range Province and adjacent regions) caused vertical motion of the basement in the study area (and, correspondingly, present accumulations of fluids, possibly, hydrocarbons). These present effects, we believe, have been "programmed" during sedimentation. A more detailed and deep analysis of the structure and the geological history of the region may render more insight into the modern dynamics of the region. 


\section{Stratigraphy}

[Adapted from the work and thesis of Anastasia Minaeva, MTU, 2001, summarized in the main body of this report. Figures that are duplicated from the main body of this report are presented here at small scale.]

\section{Evaluation of seismic attributes in Wamsutter Field, Wyoming.]}

The main target in Wamsutter field is the Almond Formation. The Almond Formation is the youngest formation (Upper Cretaceous) in the Mesaverde Group of southwestern Wyoming (Figure 2). In the subsurface, the Almond Formation can be subdivided into three genetic units:

- an upper unit which is transgressive in origin and which includes a highly productive barrier-bar facies and a coal-bearing facies deposited dominantly in back-barrier environments;

- middle unit, consisting of several dominantly marine shale-to-sandstone sequences that contain little or no coal;

- and a lower, fluvially dominated, coal-bearing unit. The upper Almond is the primary exploration target, significant gas potential may also exist in the middle and lower Almond, as it contains numerous thick coals, which have generated both oil and gas and have expelled significant quantities of gas. (Martinsen et. Al., 1995).

The Almond Formation is overlain by the marine Lewis Shale and underlain by the Ericson Sandstones. The thickness of the Almond formation in the Washakie and Great Divide Basins varies from 250 to more than $500 \mathrm{ft}$. The drilling depths to the top of Almond formation vary from a few thousand ft to over $16000 \mathrm{ft}$ in the center of the Washakie Basin (Hendricks, 1994).

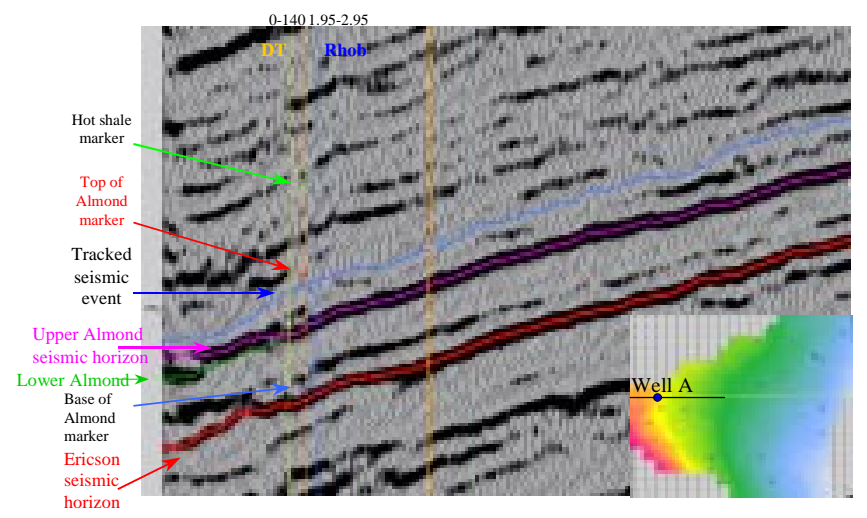
Variations in the formation thickness and in lithofacies may be due, at least in part, to syndepositional movements along basement block faults. Both its upper contact with the Lewis Shale and its lower contact with Ericson Sandstone stratigraphically rise to the west. The Almond-Ericson contact is commonly very sharp. The contact between the Almond and the Lewis is very sharp and is marked by a transgressive surface of erosion (Martinsen et. al., 1995).

Fig.2. Stratigraphic tie of reflections.

\section{Petroleum potential}

The Almond formation is one of the most important gas producing units in southwestern Wyoming. Most of the gas production in the eastern areas of the Washakie and Great Divide Basins and intervening Wamsutter arch is predominantly from the marine sandstones of the upper Almond. Within this area approximately 65 fields produce from the Almond Formation. Wamsutter field primarily produces gas. First year gas production for Wamsutter field area is shown on Figure 3. Two areal trends of production can be identified from it. A linear trend on 
the west shows the largest production from the sand barrier associated with Almond formation.

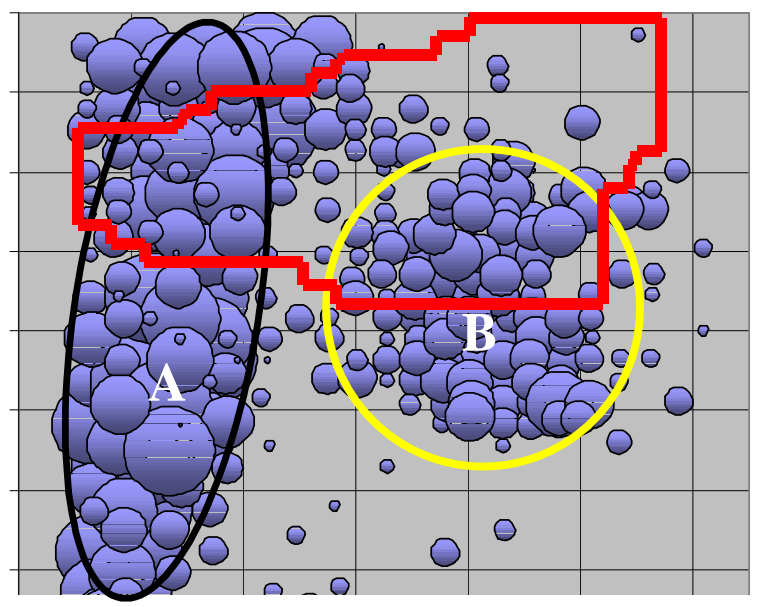

Production from zone B comes from different intervals, perhaps from the overlying shales.

Fig.3. The map of productivity in the study area.

[end of material taken from Minaeva, 2001/ the remainder of this section is original with TransSeismic International.]

\section{DFM INTERPRETATION OF SEISMIC DATA}

The general approach of interpretation of 3D seismic data acquired in given area (within the red outline (Fig.3)) is three-fold:

The structure and petrophysical parameters of producing stratigraphic units are considered (by DFM) to be due to the geodynamic conditions of basin's development. These conditions, among other factors determining reservoir properties, were very important at the sedimentation stage. Then, inheriting general pattern of existing geodynamic state, these conditions controlled general behavior of the dynamic fluid processes in the periods of generation of oil and gas pools and continue to act at present.

The spatial distribution of the anomalous pressures is controlled by modern vertical motions of the blocks of the sedimentary sequences in the "basin-basement" system.

Each localized structural or formation unit or a collection of them create an anomaly of the total pressure of a positive or a negative sign. This anomaly can be detected at the boundaries of the stratigraphic interval which contains this or these units.

Thus, the process of interpretation of 3D seismic data in the study area included the following successive stages:

Traditional seismic-stratigraphic technology targeted at correlation and stratigraphic tie of reflections within the producing interval of the basin, as well as overlying and underlying reliably correlative seismic horizons.

DFM transformation of the seismic cube aiming at calculation of relative values of the anomalous pressures in the initial scale of the cube and with the same discreteness. The result of DFM transformation includes an estimate of the relative values of the anomalous total pressure (deviations of pressure values from the normal ground pressure in each depth point) / 1,2/.

Integration of the estimates of the anomalous pressures in given intervals of the cube and construction of the maps of these estimates. The intervals were selected to include target reflectors. 
Results obtained at the above mentioned stages allowed for compilation and analysis of structural-dynamic maps for different intervals of the subsurface. Each of these maps represents a combination (overlay) of the maps of structural parameters (isochrons, gradients of isochronous surfaces, etc.) and the maps of the anomalous pressures for the same horizon or an interval of the sedimentary section comprising given structural unit or a combination of units.

Before commenting the results of DFM interpretation, let's define the main elements of the clinoform structure of Almond formation along one of east-west sections of the initial cube (Fig.4.).

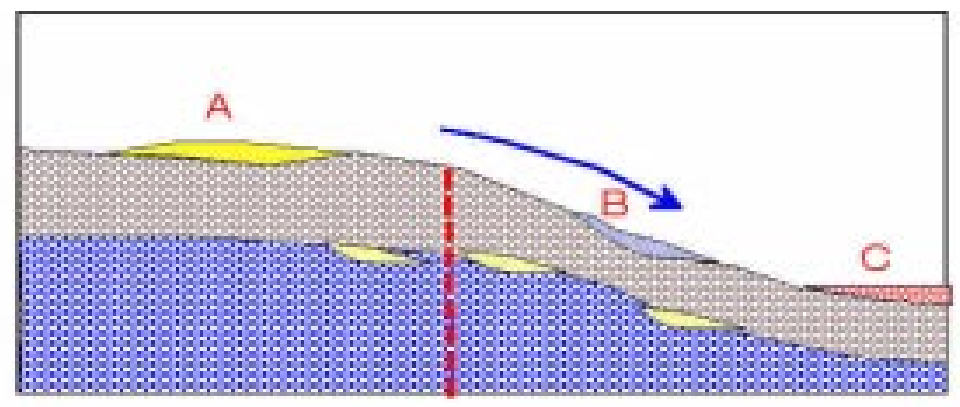

Fig.4 Vertical section of the cube and the model of clinoform deposits in the interval of Almond formation.

Taking into account the hypothesis of clinoform structure development, one can delineate typical patterns in Fig.4. In the terminology of seismic stratigraphy, these patterns allow for identification of an undaform (C), an orthoclinoform (B), and a fondoform (A). In the terminology of paleogeography, these regions correspond to three environments of deposition: deep sea, continental slope with the water depths from 50 to $200 \mathrm{M}$, and shallow sea representing a fore-deltaic plain with water depths of 25-50 m. According to available conceptions, sandy-silt sediments mainly formed on the fore-deltaic plain, and these sediments overlie the sediments of the preceding clinoform as a sheet. Isolated sandy-silt lenses were formed in the regions of steeper dips of the basin's bottom, while north-south incisions, as results of turbidites' movement, were formed in the regions of steep dips of the substrate of the sea basin. Explorationists estimate the width of the gradient zone of paleobathymetry almost identically (from 5 to $20 \mathrm{~km}$ ). The region of deep sea represented an area where condensed series and sandy-silt lens-like bodies were formed. Generation of sandy-silt lenses in a zone distant from the shoreline was connected to avalanche sedimentation.

At this stage some limited geological and geophysical information [courtesy BP Amoco] was available to us. We also considered some analogous examples of other hydrocarbon-bearing basins that we had come across in the past (first of all, a number of fields in Western Siberia). All this led us to assume (at the level of a working model which - this is very important - is open for on-line examination and correction) that the western part of this area is composed of shallowwater and very shallow-water Almond bar facies and corresponds to a fondoform region (region A). The eastern part can correspond to an undaform region, i.e. to the main area of avalanche 
deposition which occurred at more gentle dips of the sea bottom. This assumption, i.e. the lithology of Almond formation in this eastern part, should be tested by drilling. (We have no such data at present). There is a clearly visible gap (i.e. a zone of almost zero hydrocarbon potential) between the western and eastern parts (observed also in fig.3). This gap probably corresponds to an orthoclinoform region (region B). Thus, we suggest that the characteristics of the middle part are determined by the slope where dips as low as several degrees significantly limited sedimentation possibilities, since detrital material migrated into the region $\mathrm{C}$. Nevertheless, isolated sandstone bodies can be formed here (filling of underwater canyons, etc.), possibly, with good reservoir properties.

Other explanations of the nature of non-hydrocarbon-bearing region are also possible, but at the current stage of exploration we consider them less probable. For instance, if it were a deepwater area between two shallow-water areas, then, with account for the widths of three discussed areas, it would be, most likely, also filled with detrital material (with a hydrocarbon potential).

One of the possible mechanisms of clinoform generation is an oscillating dynamic mode of the basin's basement motion in the form of periodical movements of the isolated active blocks of the basin (a fault indicated by blue dotted line in fig. 4 is related to the existence of two deep active blocks of the basement).

Experience of application of DFM in such sections shows that clinoform bodies should clearly manifest themselves in the field of anomalous total pressures in a number of stable and characteristic indicators:

the regions of an undaform and a fondoform ( $\mathrm{C}$ and $\mathrm{A}$ in fig.4) manifest themselves in the form of positive pressure anomalies (decompression areas),

the transition area (zone B in fig.4 or an orthoclinoform) manifests itself in the form of a highpressure anomaly.

It should be specially underlined that the pressure anomalies are detected at the external edges of the thin interval confined by strong reflectors (the top and the base of the interval) and having internal clinoform structure. Combination of the mentioned indicators resulting from the application of DFM technology allows for a mapping of such intervals with the internal clinoform structure.

Taking into account all the said above, let's comment the results of DFM interpretation in the study area. The overall character of anomalous pressure distribution within the seismic cube can be seen in fig.5. 

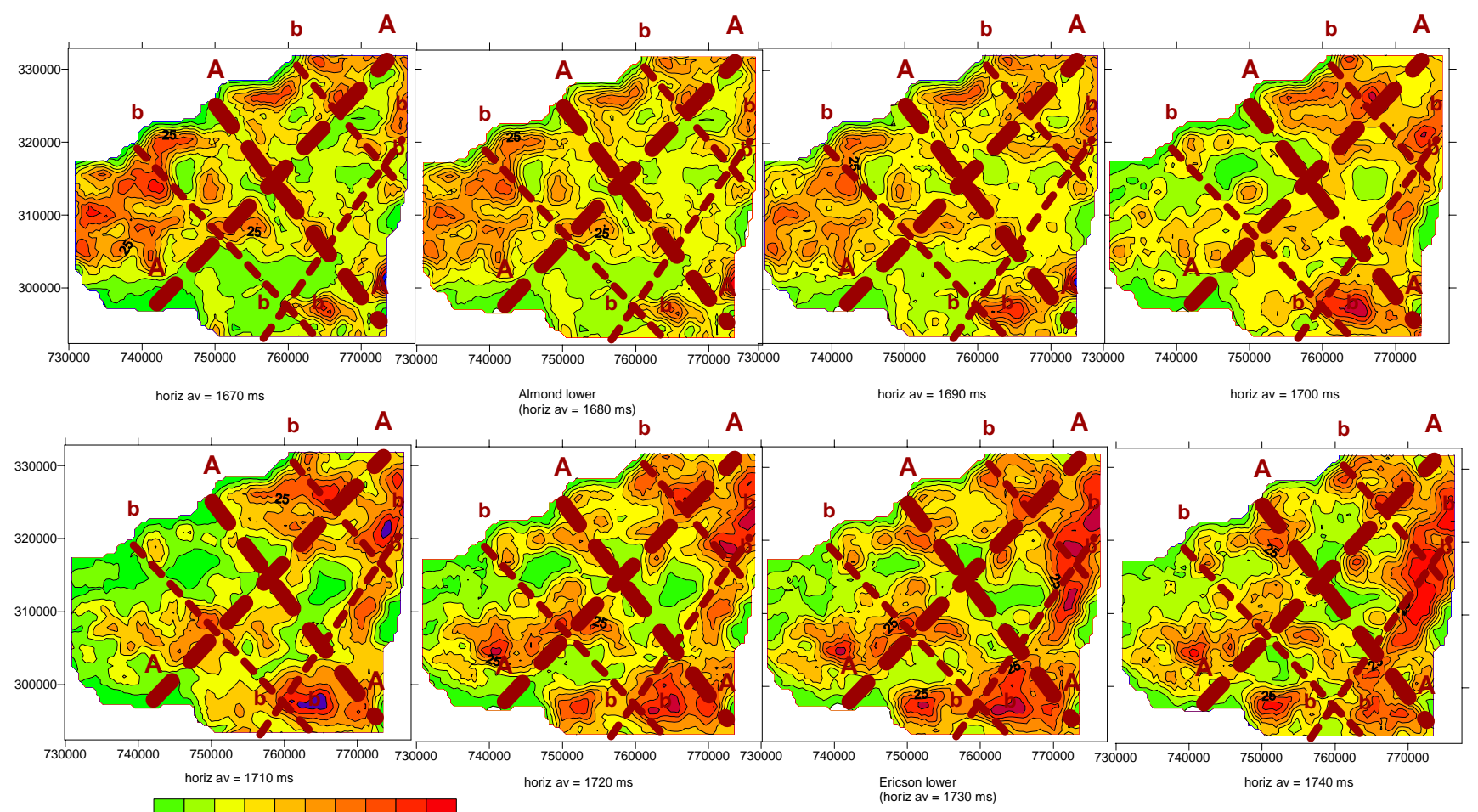

Fig.5

$\mathrm{dP} \Rightarrow \mathrm{MIN}$

Fig.5. A series of horizontal slices after DFM transformation of the cube.Color scale corresponds to relative pressure estimates.

Here one can see several horizontal slices color-coded in the estimates of the anomalous pressures. The slices are selected in the closest vicinity of the producing interval and inside it. In this case the color scale reflects relative estimates of the anomalous pressure: red tints correspond to the areas of low pressure with respect to the normal lithostatic value, while green tints indicate areas where the pressure values are close to normal ones. Analysis of this figure as well as all other slices of the seismic cube led us to the following conclusions:

a) in given case the modern dynamic state of the basin is determined by the boundaries of blocks (along the first-order line A-A which divides the region into two large blocks with different activity and several second-order blocks along the boundaries b-b),

b) the boundary between large blocks along the line A-A is confirmed by the dynamic state of lower interval of the basin in the interval from 2100 to $3200 \mathrm{~ms}$. (Fig.6. shows a map of anomalous pressure values found for the whole interval from 2100 to $3200 \mathrm{~ms}$ ),

c) two second-order blocks delineated within Almond formation possess anomalously low pressures. (The most southwestern block and the most southeastern block indicated in the fig.5 as B sw and B se correspondingly).

The location of the first-order boundary A-A and the character of division of the basin into the second-order blocks are also confirmed independently by morphology of the ground surface in the study area (see fig.1). 


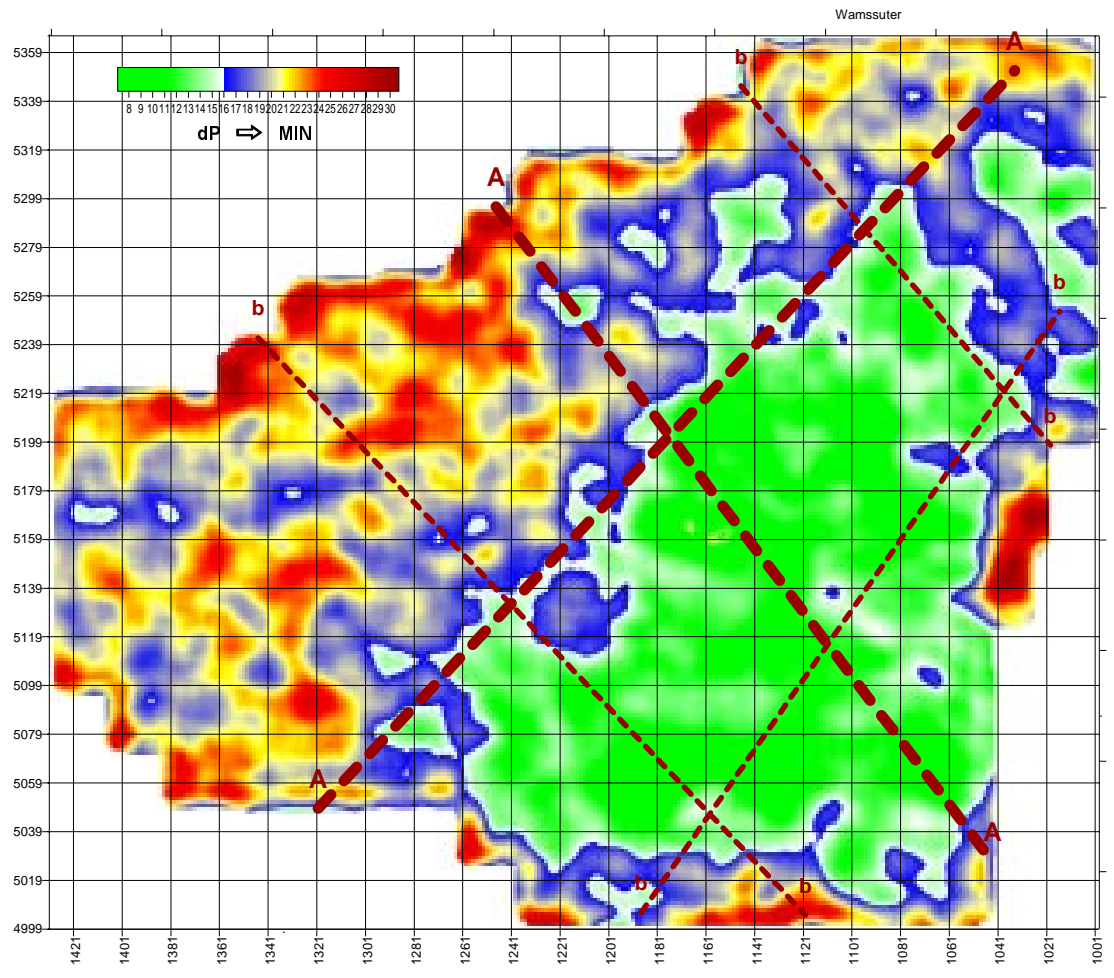

Fig. 6. The anomalous pressure map on the base of Almond formation. Characteristic distribution of the anomalous pressures allows for reliable definition of the boundary between the first-order blocks along the line A-A.

The performed analysis suggested to us an idea to calculate a combination map of the anomalous estimates on the top and the base of Almond formation, i.e. a sum of the estimates is found in two intervals: 800 $1100 \mathrm{~ms}$ and 1580-1660 ms. In essence, this gives an estimate of the anomalous pressures for the whole Almond formation. This map is shown in fig.7 where fig. 3 has been also included for

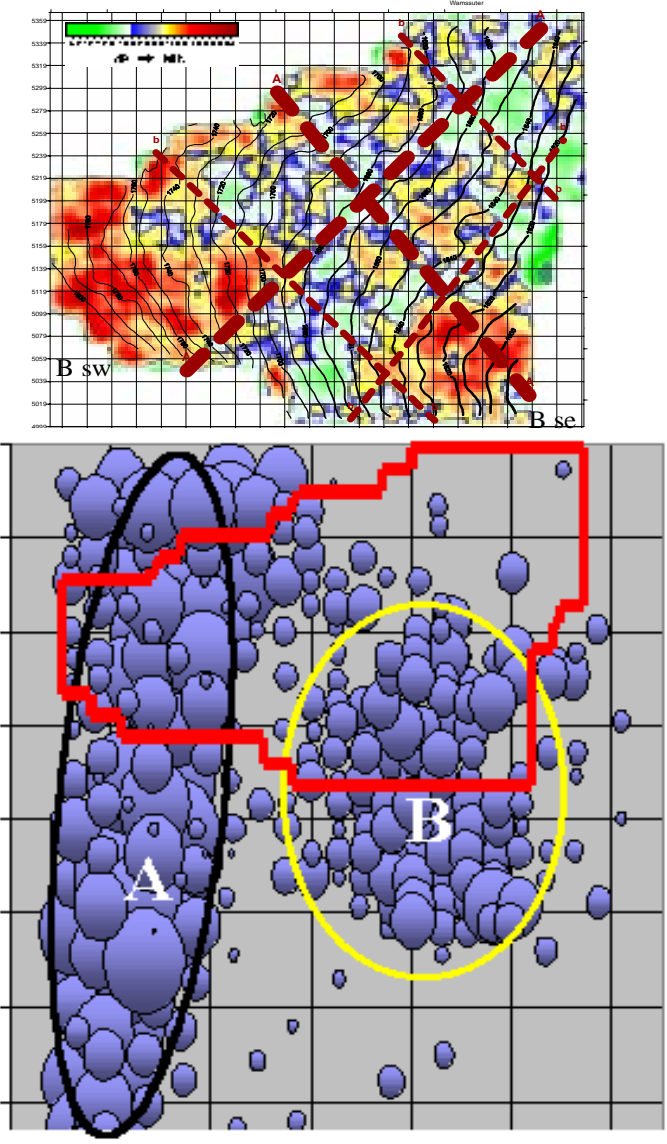
comparative analysis.

Fig. 7. . The map of anomalous pressures in the producing interval and virtual distribution of productivity of this interval estimated on cumulative production of gas (and oil). Two blocks ( $B$ sw and $B$ se) with anomalously low pressures are clearly distinguished. Location of these blocks correlates very well with virtual productivity of Almond formation.

The latter shows two zones (A and B) possessing the main productivity within Almond formation. It is evident that there is good correspondence between the blocks with maximum level of decompression and zones with maximum fluid saturation (including oil).

A more detailed representation on the fluid saturation distribution within the formation under study can be obtained even in more narrow time intervals where (as was discussed above) anomalous pressures can depend on local lithological heterogeneities originating from the clinoform mechanism of deposition. Fig.8."a" shows such a map for the interval of 1580-1660 
ms, while Fig.8"b" shows a modification where the zones of higher than normal pressures are defined. In this case the local zones of increased pressures should correspond to the subsurface features with higher values of the elastic moduli, i.e. to sand bodies.
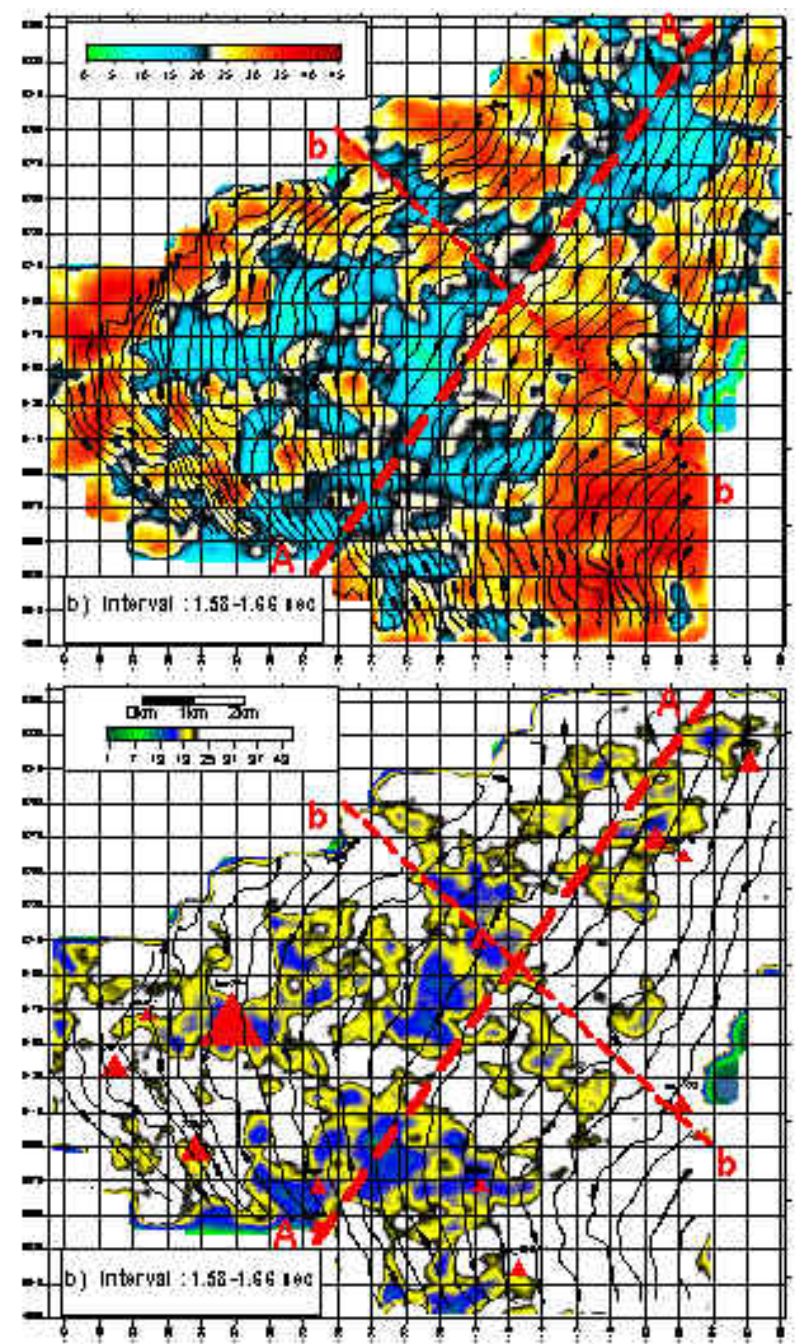

Fig8. The map of anomalous pressures in a narrow zone inside Almond formation ("a") and the map of local pressure anomalies connected to sand bodies (" $b$ "). Conventional signs are used to mark the wells which penetrated local reservoir bodies with oil inflows (the symbol size corresponds to the volume of produced hydrocarbons).

If we also post the locations of wells in which oil inflows were obtained in given depth interval and sand beds were penetrated, then, we hope to see good correspondence between the zones of anomalous pressures and actually proved productivity in this interval.

\section{CONCLUSION.}

We believe that the limitations of traditional technologies which are related to seismic resolution of stratigraphic features in terms of spatial dimensions and properties can be solved through the application of the Dynamic Fluid Method and its approach to the interpretation of seismic data.

The DFM strategy is based, firstly, on the amplitude parameters of the seismic signals, and secondly, on the integral characteristics of present stressed state of the earth. In combination with the classical approaches, an independent estimation of the anomalous pressures provides sufficiently objective basis for the construction of the scheme of the basin's block dynamics and local prediction of the occurrence of stratigraphic features of small size (with respect to the seismic wavelength). The scheme of block dynamics and local prediction of the pressures independently of the reasons and mechanisms of perturbations in the field of stressed state of the basin are closely connected to the parameters of modern dynamic fluid processes and, consequently, to virtual productivity of the reservoirs under study.

These special features of the DFM technology allowed for a determination of the general character of fluid saturation in the producing interval in the study are and for the detection of local subsurface features with promising reservoir properties. 


\section{The Dynamic Fluid Method (DFM) analysis of seismic data in the area of Waha fields.}

Pisetski V.B. Patrushev Yu.V. and Voronin O.A

Trans Seismic International, Inc.

\section{INTRODUCTION}

From the report compiled by Bob A.Hardage, R.P.Major, and Tucker F.Hentz /1/ the following summarizes some of the main features of the Waha fields:

- Productivity of the Ellenburger carbonate reservoirs is mainly determined by the tectonic elements of geological section (faults, fractured zones, etc.) and, in fact, does not depend on the porosity of the rock matrix and the structural factors.

- Dynamic-fluid parameters, derived from the well data, widely vary (pressure, flow rates, etc.).

- No reliable link has been found between velocity and amplitude, in this case.

In given conditions, it's possible to use an approach based on the positions of modern dynamics of the sedimentary basin. Parameters of the modern dynamics should essentially control the reservoir parameters, in our opinion. The history of tectonic processes is complex as the intensity and the direction of forces which successively destroyed the sedimentary cover may have varied dramatically. But we know that in given moment of time a definite slow (plicative) process of motion of all subsurface blocks takes place in one direction or another. For instance, if a bending force is directed from the basement toward the base of the sedimentary cover in given moment of time (to date), then a totality of the blocks is activated so that their sides will coincide with one of the main axes of bending deformation. Tomorrow (we mean quanta of the tectonic time) the axis of bending will rotate, and another system of blocks passes into the active state, while the previous system of blocks recovers normal lithostatic pressure.

It is natural to assume that a reservoir will have the highest value of productivity at the boundaries of blocks with different modern activity (on the boundary of blocks moving in different directions). The processes of destruction of the rock mass (generation of new fractures and renewal of old ones with a mineral filler which in our case was formed, for example, in the process of dolomitization of carbonate rocks) are activated along and in the vicinity of these boundaries.

Thus, the problem of estimation of relative values of the ground pressure can become a valid purpose of seismic data interpretation. If this problem is solved, then the boundaries of the active blocks will become "visible". These blocks can be constructed based on the characteristic spatial variability of the anomalous values of the ground pressure within the analyzed interval of the sedimentary cover or the basement. In other words, an estimate of actual variability of the ground pressure in the studied volume of the subsurface gives us an opportunity to separate the system of all faults, visible and invisible for interpreter's eye, into "old" and "new" faults. In given context the difference between a new fault and an old one lies in a relatively contrasting change in the stressed state at the boundaries of blocks in current (modern) tectonic period, while adjacent blocks separated by an old fault are presumed to have the same sign and value of the stress. It should be underlined that discrete media behave as a quasi-liquid under action of slowly 
changing external forces/ and the tensor of stresses can be replaced with an estimate of the ground pressure with a sufficient accuracy on condition that the relation $\tau \leq 0.75 \sigma$ is satisfied / 5 /; where $\tau, \sigma$ are tangential and vertical components of the stresses.

Taking all of the above into account, further, we will use the term pressure. Of course, we mean the relative value of the pressure with respect to normal (lithostatic) value. And it is assumed that the values of the DFM parameter present an estimate of the decompression state with respect to the normal pressure, and the higher the values of DFM parameter, the higher the degree of decompression. It is the parameter found with the aid of DFM technology will present the target parameter for prediction of the scheme of modern block dynamics in the Waha area.

\section{THE METHODOLOGY OF DFM ANALYSIS AND THE RESULTS OF PREDICTION OF THE SCHEME OF BLOCK DYNAMICS IN THE WAHA FIELDS.}

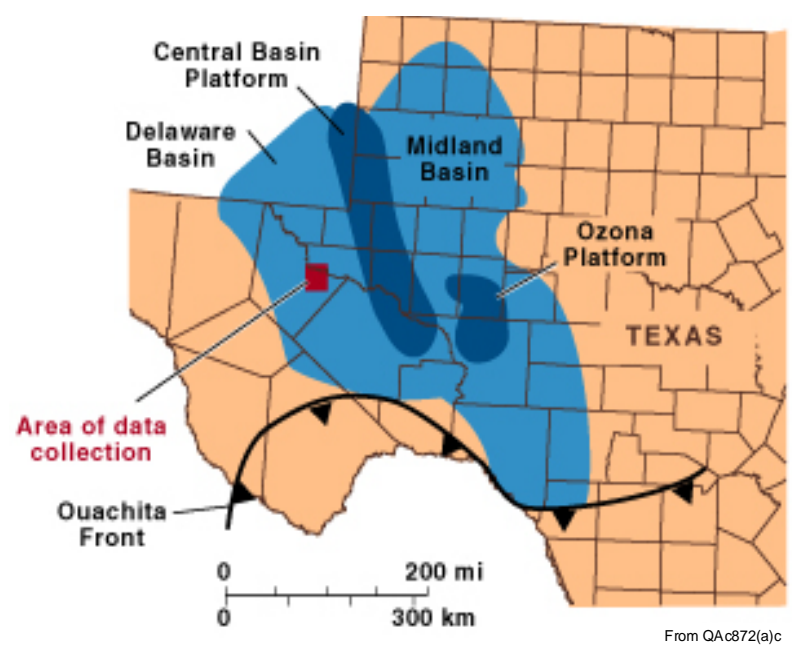

\section{Figure 1. Location map of the study area.}

The study area is located within the Delaware Basin (Fig.1). This figure also shows a part of the area where 3D seismic data are available in the form of a migrated cube, plus all the geological, geophysical, and production data from the report /1/. This collection of data has served as a basis for DFM interpretation. The boundaries of this part of the 3D seismic area and the faults were identified as a result of an integrated interpretation of all available geological and geophysical information and taken from the report /1/ and have been shown in Fig.2 representing the structure of the Ellenburger horizon. And the authors of the report

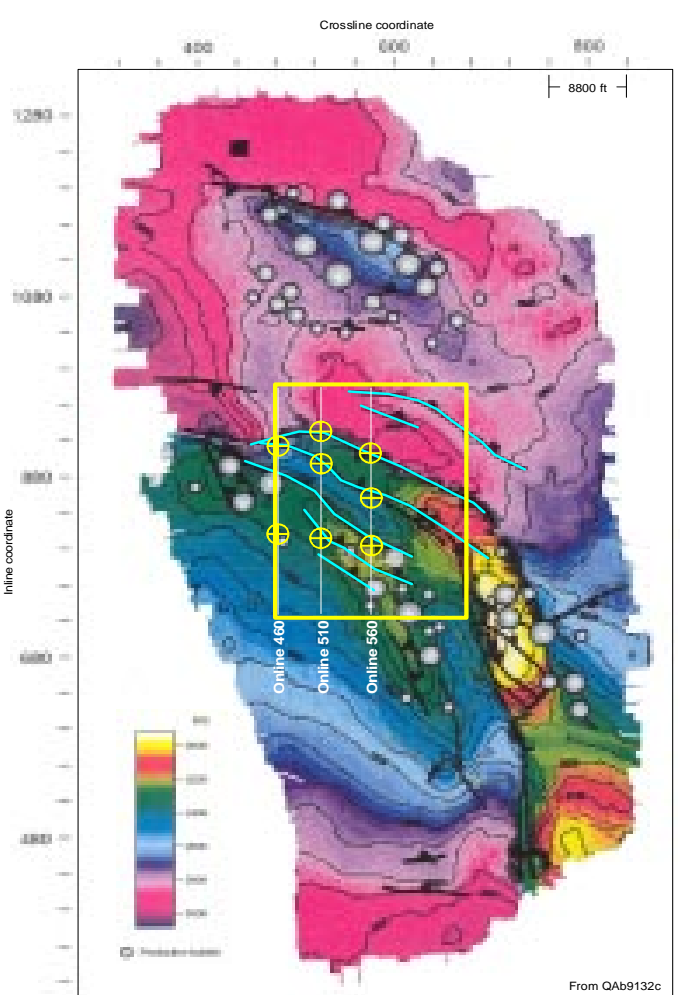

Final Technical Report October, 2002 separate the two groups of faults further indicated by different colors. The solid red lines represent faults coincident with sharp changes of the structure and geological section (we will call them the faults of the first group), while solid green lines represent faults crossing the first-group faults in approximately orthogonal direction (the second group). All these faults are shown in all of the figures.

Figure 2. The structure map on the Ellenburger horizon and the outline of the $3 D$ seismic area.

The general tectonic setting of the study basin is illustrated by the seismic section along the online 510 shown in Fig.3a. According to the stratigraphic tie, the boundary of the last high-amplitude tectonic pulse is coincident with the Mississippian interface. After this pulse, plication processes are dominating in the basin until now. This conclusion follows from the general comparative analysis of the geometry of Bone Spring 
and Mississippian interfaces. Consequently, all estimates of the DFM parameter (pressure) in the depth interval from the ground surface down to the Mississippian interface should reflect, on the one hand, the spatial character of the modern stressed state of the basin in a pure form, and on the other hand, the influence of a strongly uneven topography of the Mississippian interface. In the deeper intervals, the modern stressed state will be determined by a number of mechanisms, e.g. by variable thickness of the overlying sequences, by faulted structure of the depth intervals starting from the Mississippian interface, by different modern activity of the depth intervals below the Ellenburger interface, and others. Fig. 3b shows a DFM section for the same online 460. This DFM section is color-coded by relative estimates of the pressure, and these estimates were found from the attributes of seismic signals. Here, we see a situation typical for the whole data volume, and it can be characterized by the following:

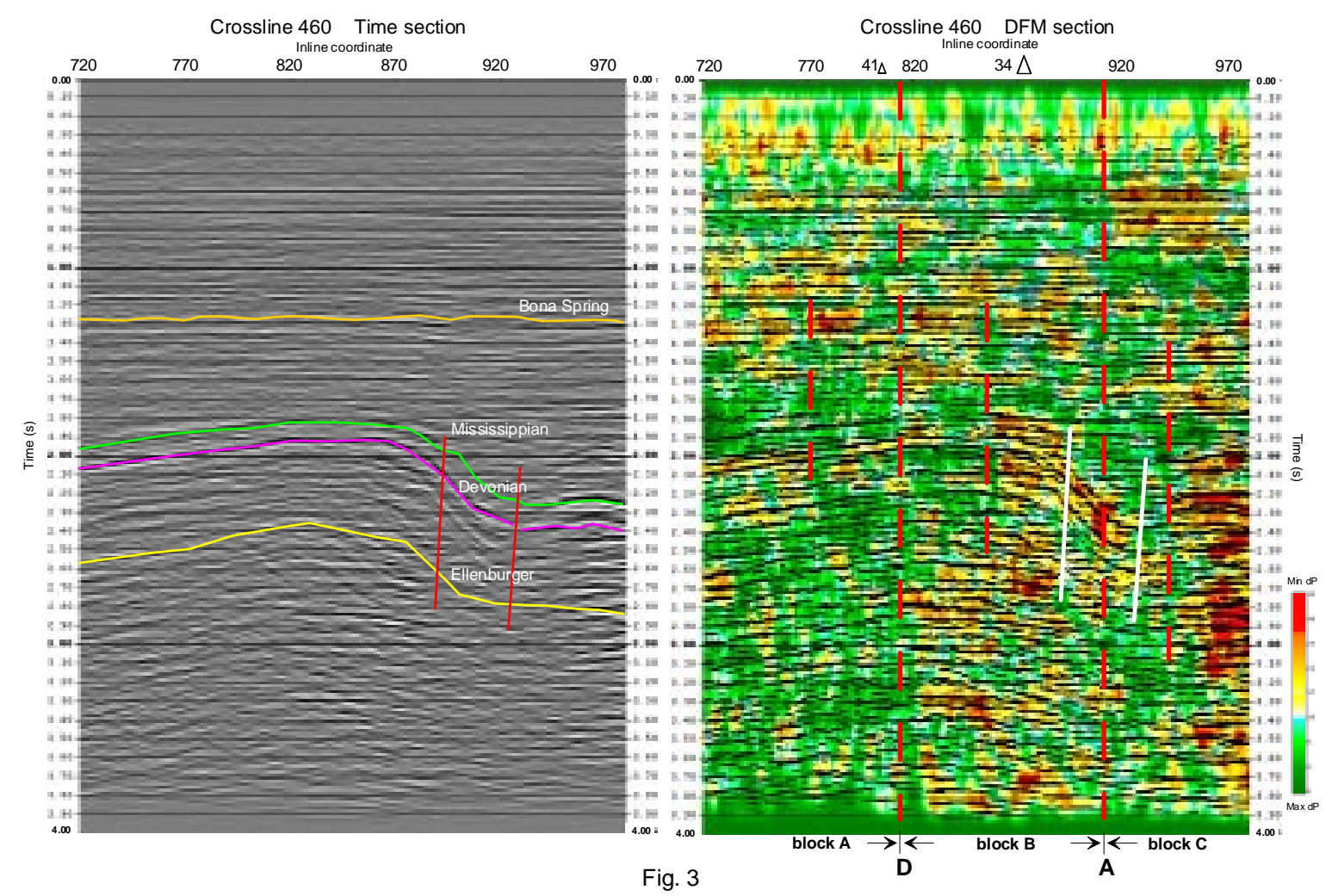

Figure 3. Stacked seismic section on line 460 and DFM section for the same line.

The highest modern activity in the basin manifests itself on three interfaces, i.e. Upper, Bone Spring, and Mississippian interfaces.

Activity in the upper intervals is determined in the vertical direction by the activity in all underlying depth intervals. Such vertical boundaries of two blocks, with moving in different directions can be identified, for example, along the lines A and B (these boundaries are shown in the figure by a red dotted line).

The same situation is observed in all vertical and horizontal sections of the data volume. Figs. 4 and 5 show two sections for online 510 and 560, while Fig. 6 shows a series of horizontal slices in the interval from 2000 to $2520 \mathrm{~ms}$. The faults detected as a result of integrated interpretation of all available information and extracted from the report /1/ are posted in all mentioned figures. 


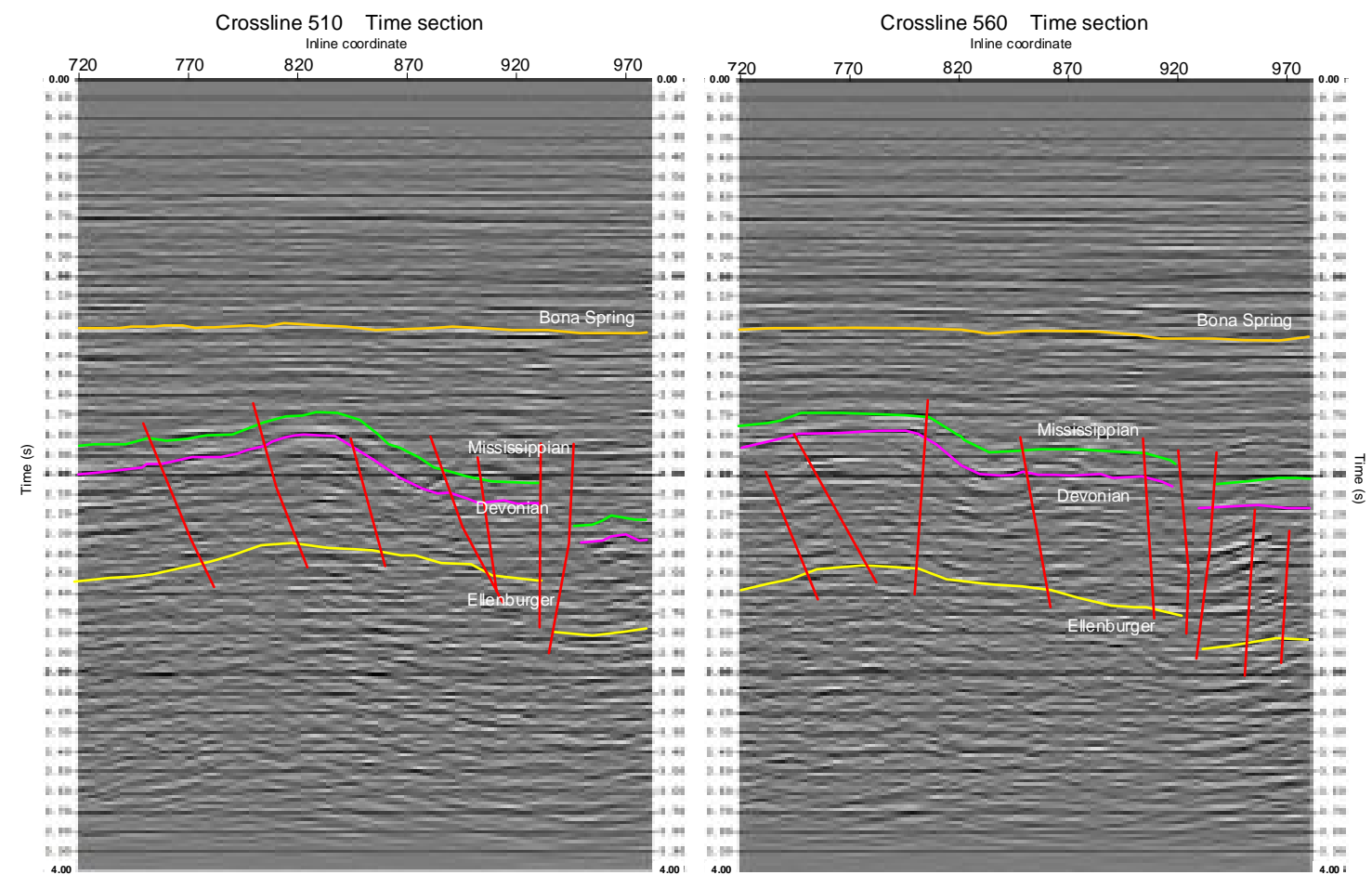

Fig. 4

Figure 4. Stacked seismic sections on lines 510 and 560.

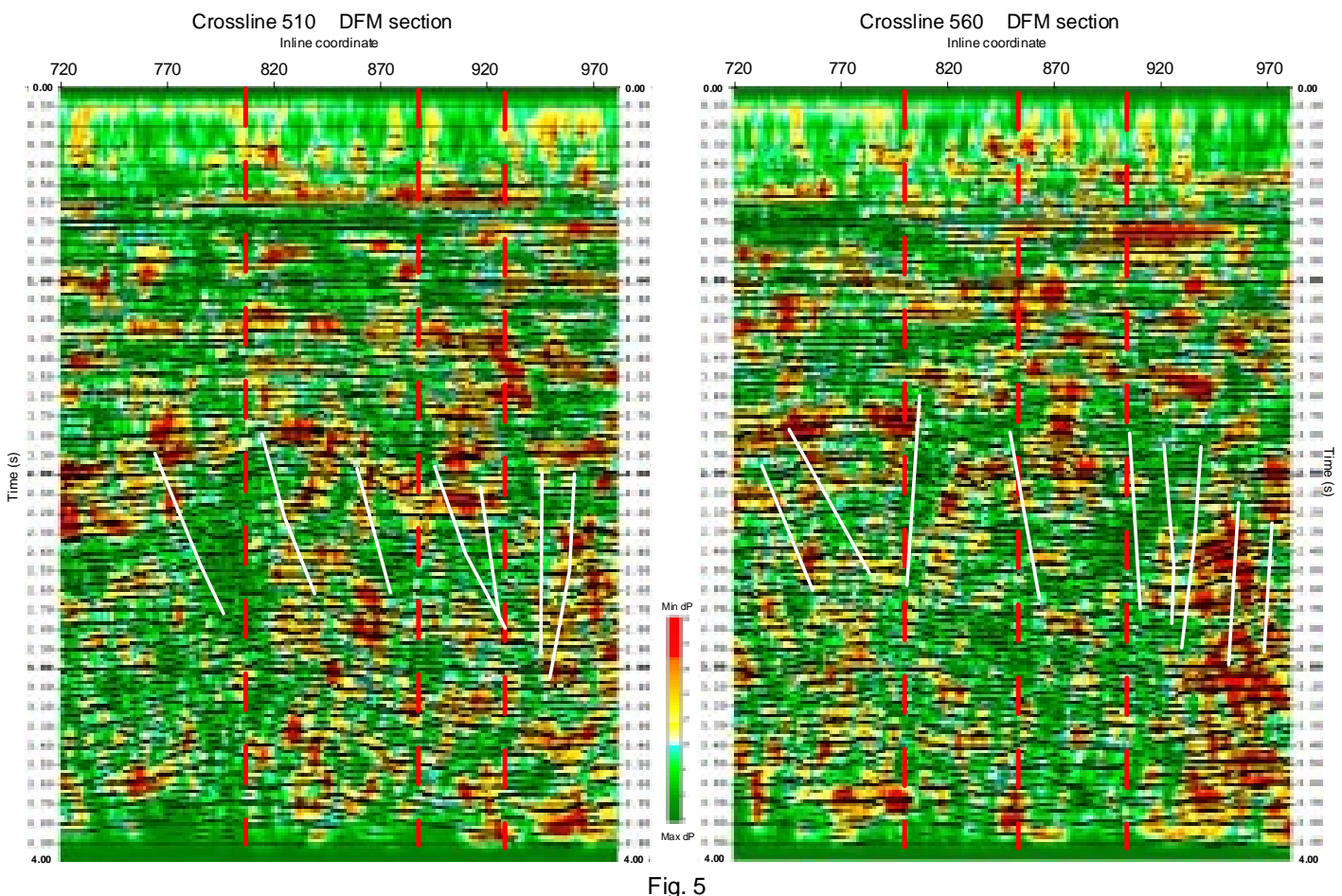

Figure 5. DFM sections on the lines 510 and 560. 

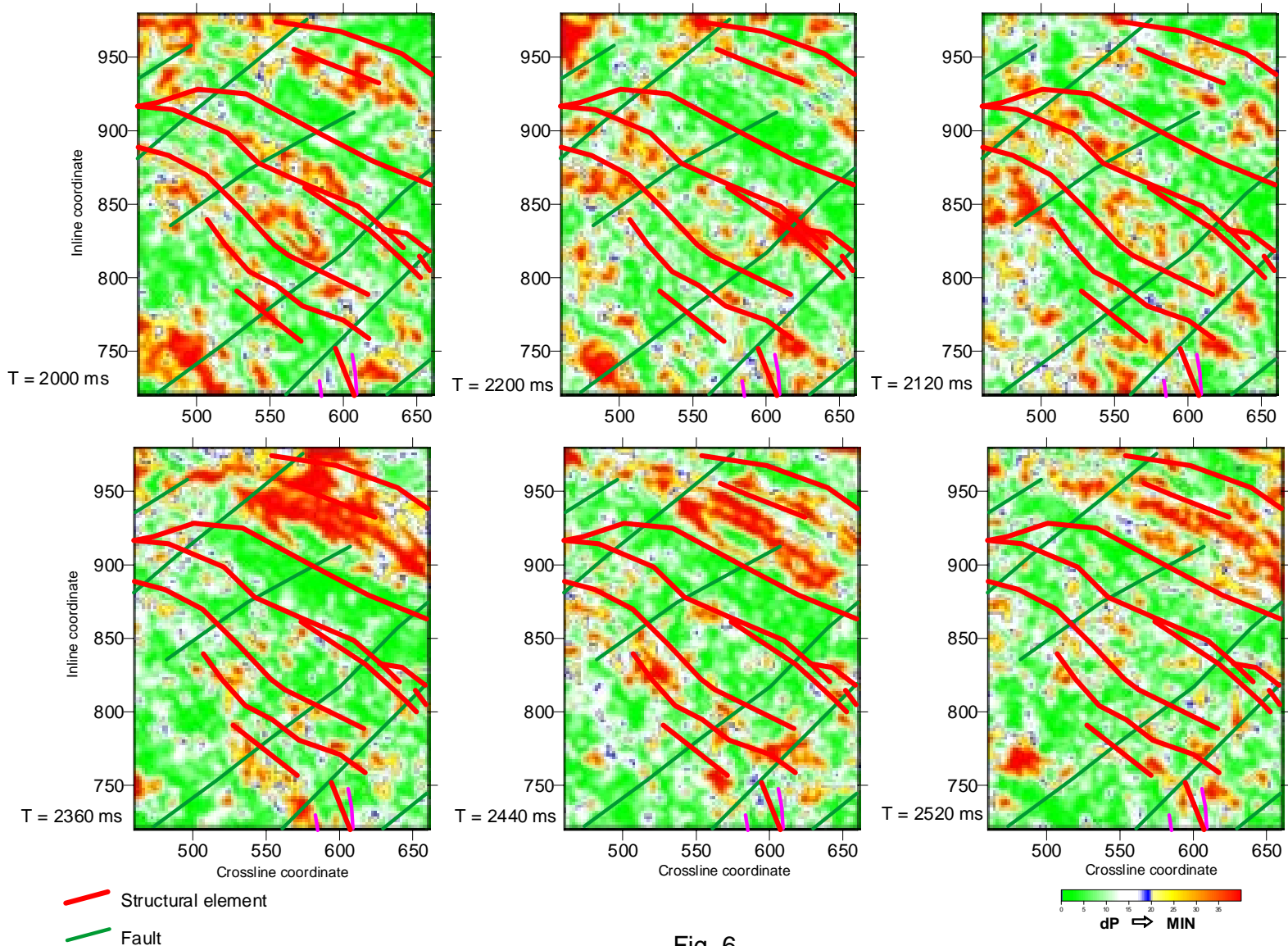

Fig. 6

Figure 6. Horizontal slices of the DFM cube in the interval from 2000 to $2520 \mathrm{~ms}$.

A conventional sign was used to indicate crossings of the boundaries of active blocks (lines A, B, etc.) with vertical sections along the lines 410,510, and 560 (Fig.2). As we see, these boundaries are in good coincidence with faults (Fig.2-6), and besides, the character of the spatial distribution of anomalous pressures in the horizontal sections (Fig.6) reveals all detected faults with different degree of contrast. Hence, we can agree that the spatial pattern of the anomalous pressures reliably control the scheme of fault tectonics defined through classical methods of visual interpretation of seismic and other data. But it should be noted here that the anomalous pressure values are an essential supplement to such tectonic schemes and can be used as a basis when taking decisions on/about the modern dynamic state of those blocks of the sedimentary cover into which it is separated by previous and modern tectonic processes.

Activity of the blocks should be analyzed, in our opinion using integrated estimates of the anomalous pressures in the intervals of the sedimentary cover related to different tectonic periods. In this case we have a possibility, on the one hand, to obtain stable estimates of the anomalous activity regions within target stratigraphic intervals in a certain order (from bottom to top or from top to bottom), and on the other hand, to define more accurately the spatial location of the vertical boundaries of the blocks possessing different degree of activity. 


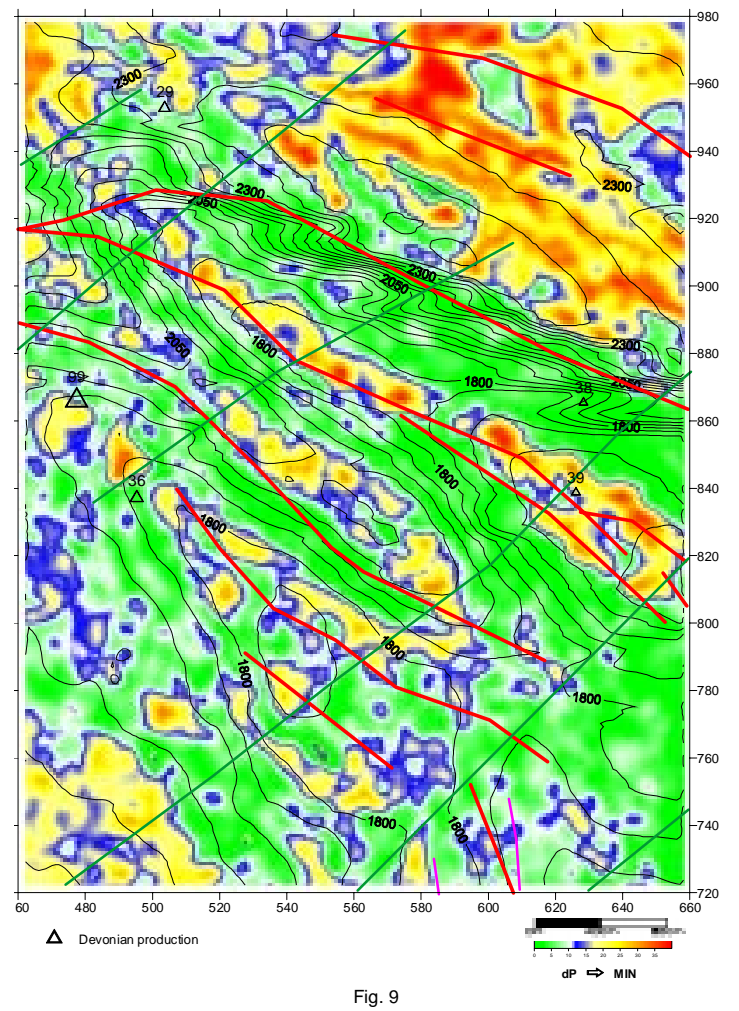

Figure 8. Integrated estimate of the anomalous pressures in the interval from 2000 to $2400 \mathrm{~ms}$.

Fig.8. shows an integrated estimate of the pressures in the interval from 2000 to $2400 \mathrm{~ms}$ with equalization for the Devonian horizon (from this horizon and below it). This map illustrates relations between linear zones of the anomalous pressures and a system of the faults of the first group. In this case, producing wells penetrate the Devonian and Ellenburger horizons located in the decompression regions (red zones), which is logical with account for the activity of given interval, and does not contradict to the assumptions of the authors of report / $1 /$ on the leading role of the faults in the distribution of productivity of this or that stratigraphic interval. The distribution of pressures varies weakly in a more narrow interval comprising the Devonian reflecting horizon (Fig.9). The faults become more apparent, particularly in the center of the area. In our opinion, this fact should attract attention

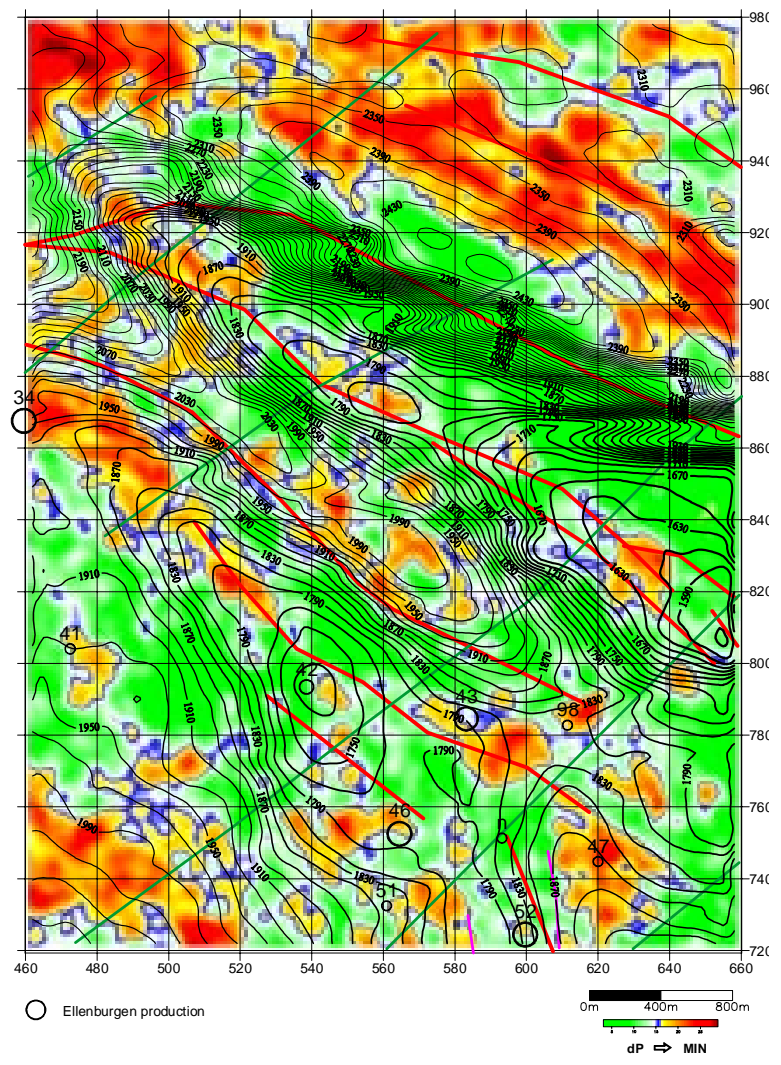

Fig. 8 in view of the outlook of drilling an appraisal well. There is a well (No.39) in this area with an 
insignificant oil inflow. The rest of the producing wells (No.29, 90, 36) in this area are located within the southern linear active zone.

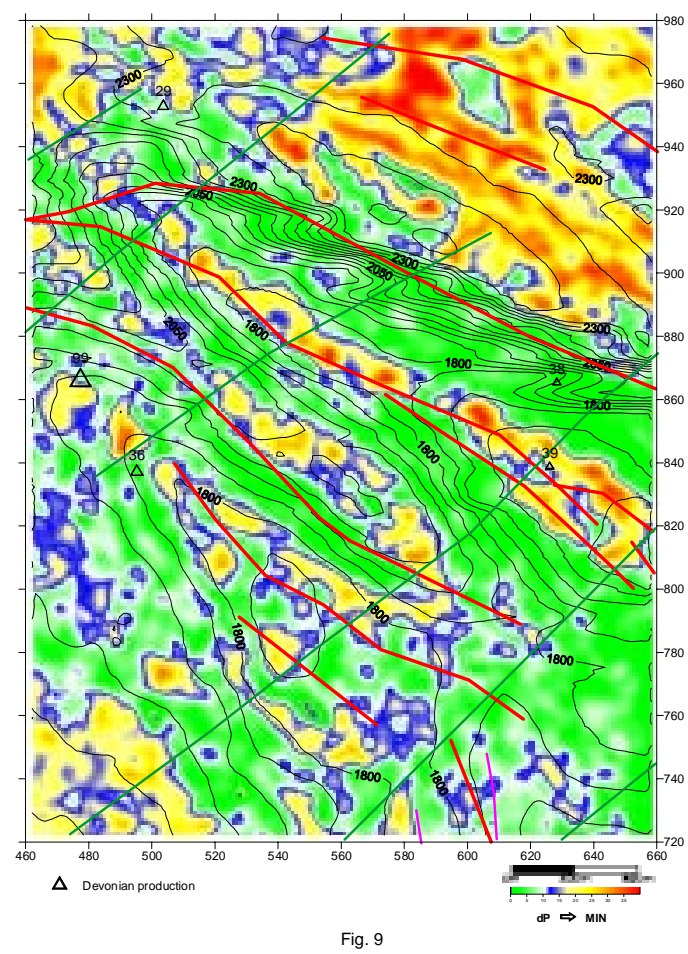

Figure 9. Estimate of the anomalous pressures on the Devonian horizon.

Distribution of the anomalous pressures within the main producing interval comprising the Ellenburger interface is shown in Fig.10. Here the estimates correspond to a large interval of the sedimentary cover - from the Ellenburger interface to the traveltime of $3500 \mathrm{~ms}$. Two main decompression areas can be defined in the southern half of the area, and two main groups of the producing wells are located exactly in this areas. In a more narrow interval (Fig.11) coincident with the Ellenburger interface, the features of the pressure distribution are localized along the faults of the first group and, in fact, this situation explains the distribution of the productivity.

Figure 10. Integrated estimate of the anomalous pressures in the interval from 2440 to $3520 \mathrm{~ms}$.
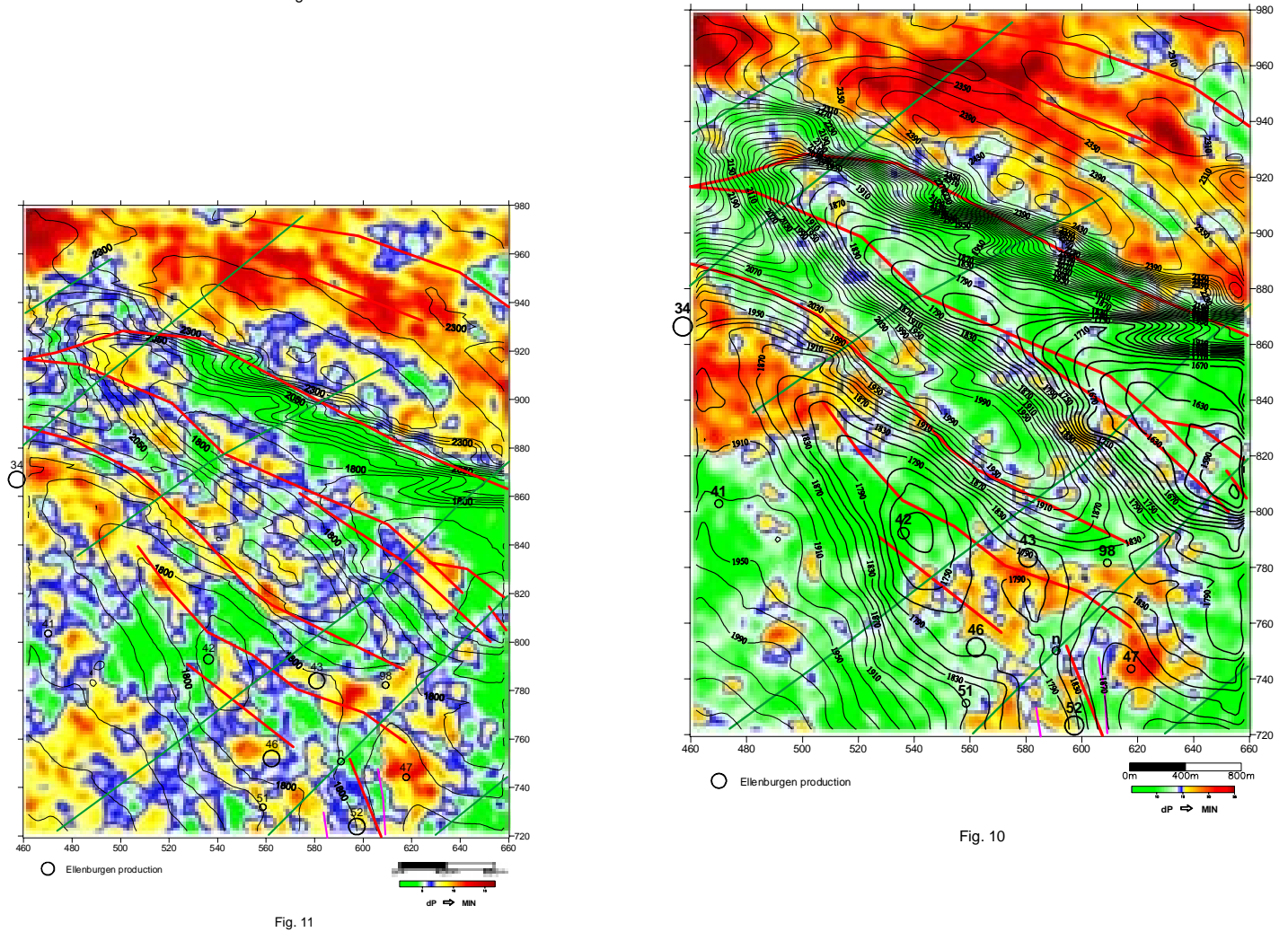

Figure 11. Estimate of the anomalous pressures on the Ellenburger horizon. 


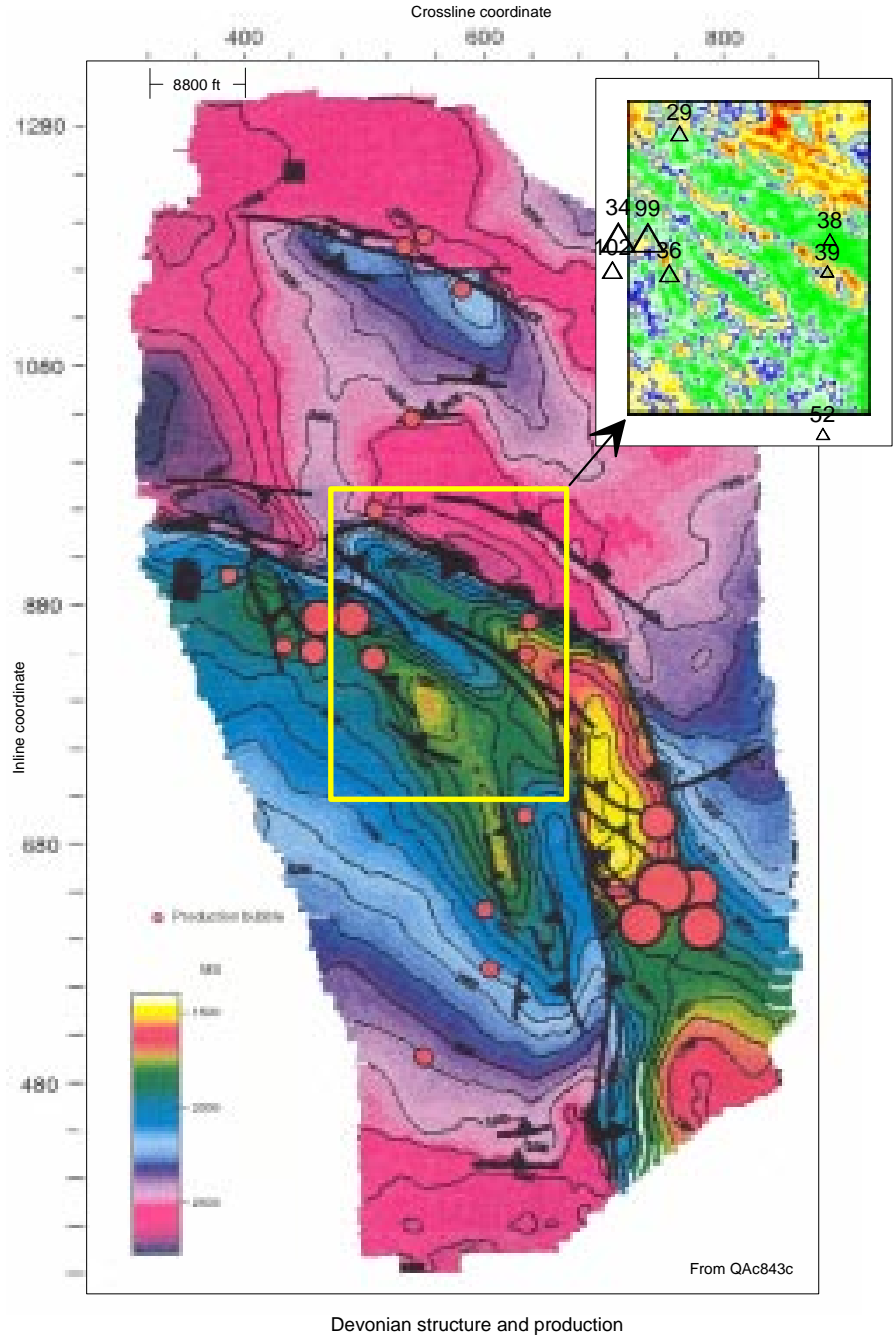

Fig. 12

Figure 12. Structural map on the Devonian horizon, and the estimate of anomalous pressures for this horizon.

Figure 13. Structural map on the Ellenburger horizon, and the estimate of anomalous pressures for this horizon.
Figs.12 and 13 are obtained by superposing the anomalous pressure maps shown in Figs. 9 and 11 on the structural maps on the Devonian and Ellenburger horizons. Here, one can see typical features of distribution of the pressures in the main intervals of the geological section and correspondence of these features to actual data on the productivity of wells.

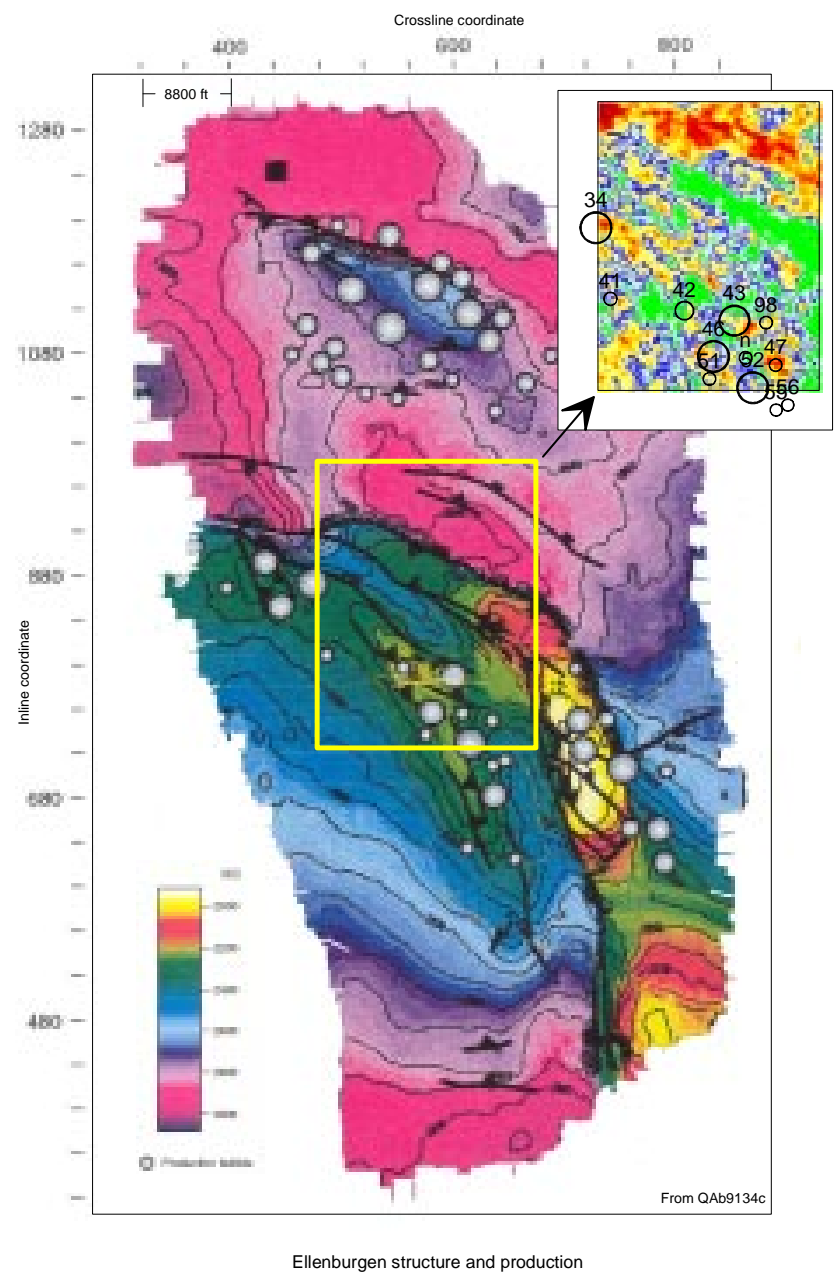

Fig. 13

The next map of anomalous pressures is shown in Fig.14 and it seems most illustrative. This map illustrates an integrated estimate of the anomalous pressures for the whole sedimentary sequence lying below the Mississippian interface (the interval from 1600 to $3600 \mathrm{~ms}$ ). In essence, this interval was formed during tectogenesis and, most likely, reflects the last, modern, process of the basin's dynamics. Here, we see quite natural separation of the whole sedimentary sequence into the blocks of certain configuration, and this system of the blocks is in agreement with the system of the faults of the first and second group. Such a map allows us to draw the boundaries of the 
active blocks, and these boundaries are posted in given figure by blue dotted lines denoted by

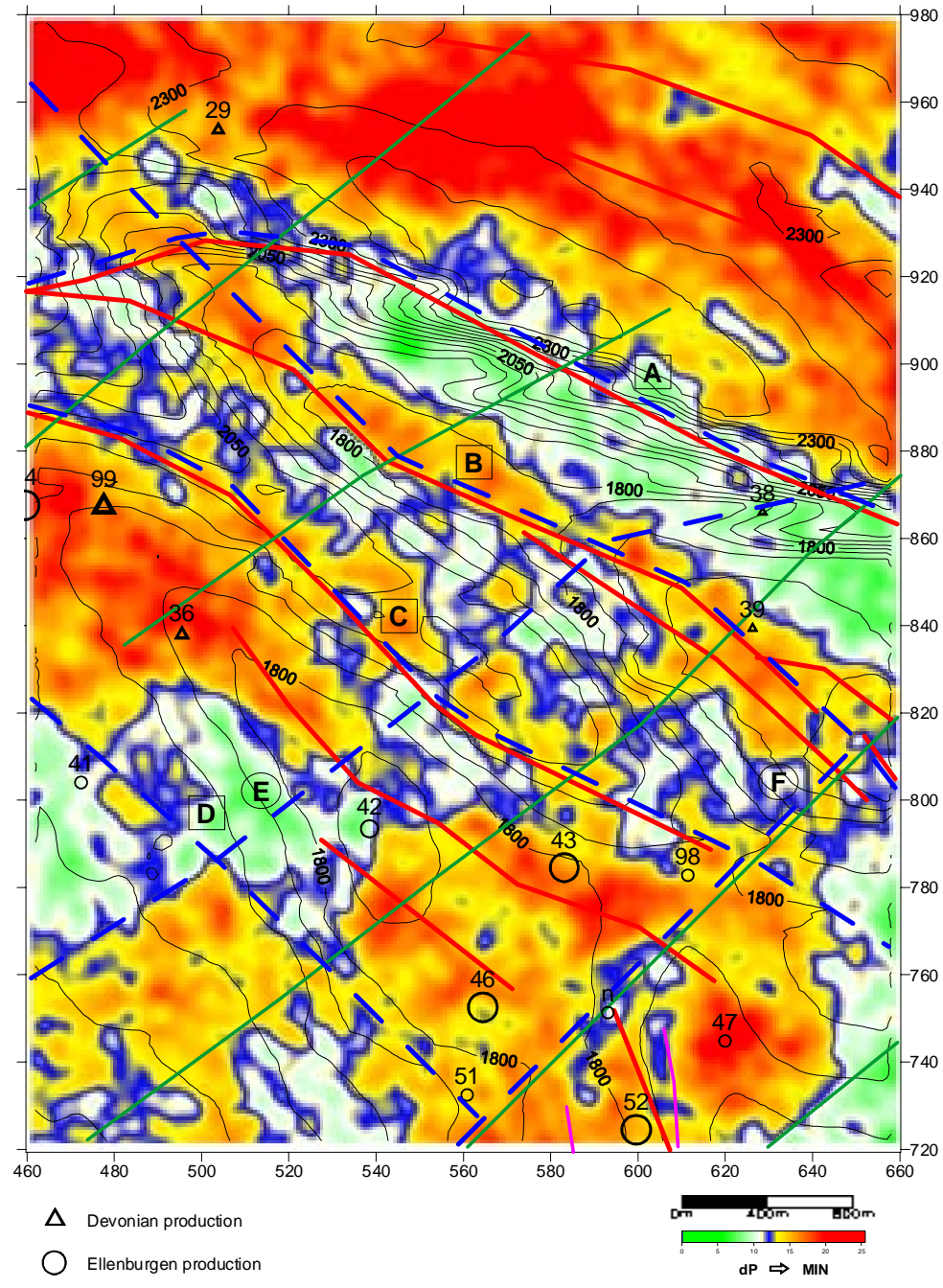

Fig. 14 letters from A to F. A number of the boundaries of the active blocks in the mentioned map are coincident with the faults of the first and second groups, thus testifying to an independent confirmation of validity of the anomalous pressure estimates. In other words, the map of integrated estimates of the anomalous pressures for the whole target interval of the sedimentary cover is an objective estimate of its productivity in wide sense and allows to correctly build a system of faults of different ranks. Hence, if we set the problem of building a scheme of fault tectonics with estimation of its relative activity in the modern period, then the DFM technology discussed here allows to successfully solve this problem. And as we see, the system of faults is guided first of all by the system of the blocks of different activity which follows from the laws of mechanics of deformable bodies with discrete structures.

Figure 14. Integrated estimate of the anomalous pressures in the interval from 1600 to $3600 \mathrm{~ms}$, and the scheme of active block dynamics in the basin.

\section{CONCLUSIONS.}

Application of the DFM technology in the given area allows to obtain a sufficiently reliable scheme of the active dynamics of the sedimentary cover. This, in turn, gives an opportunity to independently assess prospectivity of different intervals of the sedimentary cover for delineation of producing zones.

In essence, a map shown in Fig.14 can be used as a physical basis for definition of the main tectonic elements of the basin, as well as for assessment of the productivity of this or that interval of the sedimentary cover.

In given case the anomalous pressure maps obtained for intervals and individual horizons can be used as recommendations for determining new drilling sites along the most contrast boundaries of the blocks - for example, lines B, C, and E. 


\section{REFERENCES}

1. Bob A.Hardage, R.P.Major, and Tucker F.Hentz : Integrated Strategies for Carbonate Reservoir Reserve Growth: An Example from the Ellenburger Group, Permian Basin, West Texas. Final report, 1998. Bureau of Economic Geology. The University of Texas at Austin

2. Pisetski V.B Method for Determining the Presence of Fluids in a Subterranean Formation, Application for utility patent, U.S. Patent and Trademark Office, EMO 57274744, $1997 \mathrm{yr}$.

3. Pisetski V.B., The dynamic fluid method. Extracting stress data from the seismic signal adds a new dimension to our search, The Leading Edge, September 1999, SEG, USA

1. Dr. Wayne Pennigton (report) .........

2. Byerlee J.D. Friction of rocks. Pure Appl.Geophys.,116,615-626, 1978 